\title{
Analysis of MIRO/Rosetta Data
}

\author{
Dissertation \\ zur Erlangung des mathematisch-naturwissenschaftlichen Doktorgrades \\ "Doctor rerum naturalium" \\ der Georg-August-Universität Göttingen \\ im Promotionsprogramm PROPHYS \\ der Georg-August University School of Science (GAUSS)
}

\author{
vorgelegt von \\ David Marshall \\ aus Norwich, Vereinigtes Königreich
}

Göttingen, 2018 
Betreuungsausschuss

Dr. Paul Hartogh

Max-Planck-Institut für Sonnensystemforschung, Göttingen

Prof. Dr. Stefan Dreizler

Institut für Astrophysik, Georg-August-Universität Göttingen

Mitglieder der Prüfungskommision

Referent: Dr. Paul Hartogh

Max-Planck-Institut für Sonnensystemforschung, Göttingen

Korreferent: Prof. Dr. Stefan Dreizler

Institut für Astrophysik, Georg-August-Universität Göttingen

Weitere Mitglieder der Prüfungskommission:

Prof. Dr. Ulrich Christensen

Max-Planck-Institut für Sonnensystemforschung, Göttingen

Prof. Dr. Ariane Frey

II. Physikalisches Institut, Georg-August-Universität Göttingen

Prof. Dr. Thorsten Hohage

Institut für Numerische und Angewandte Mathematik, Georg-August-Universität Göttingen

Prof. Dr. Andreas Pack

Geowissenschaftliches Zentrum, Georg-August-Universität Göttingen

Tag der mündlichen Prüfung: 19.12.2018 


\section{Bibliografische Information der Deutschen Nationalbibliothek}

Die Deutsche Nationalbibliothek verzeichnet diese Publikation in der Deutschen Nationalbibliografie; detaillierte bibliografische Daten sind im Internet über http: //dnb . d-nb . de abrufbar.

ISBN 978-3-944072-65-4

uni-edition $\mathrm{GmbH} 2019$

http://www.uni-edition.de

(C) David Marshall

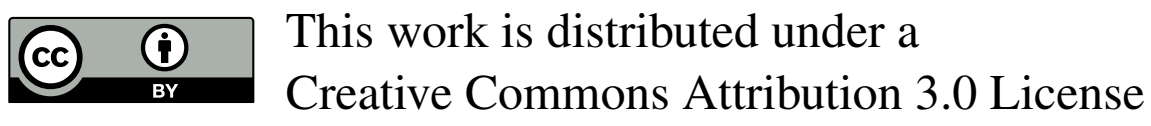

Printed in Germany 



\section{Contents}

$\begin{array}{ll}\text { Summary } & 9\end{array}$

$\begin{array}{ll}\text { Zusammenfassung } & 11\end{array}$

1 Introduction $\quad 13$

1.1 Comets .............................. 13

1.2 Observational history: from the stone age to the space age . . . . . 16

1.3 The Rosetta mission . . . . . . . . . . . . . . . . . . . . . . 21

$1.467 \mathrm{P} /$ Churyumov-Gerasimenko . . . . . . . . . . . . . . . . 24

1.5 The Microwave Instrument for the Rosetta orbiter . . . . . . . . . . . . 28

1.6 MIRO aims, results and spectra . . . . . . . . . . . . . . . . . 32

1.7 Thesis aims .......................... 36

2 Theoretical concepts $\quad 37$

2.1 Haser model . . . . . . . . . . . . . . . . . . . 37

2.2 A two level atom . . . . . . . . . . . . . . . . . . . . . . . . . . . . . . . 37

2.3 Optical depth . . . . . . . . . . . . . . . . . . . . 39

2.4 Radiative transfer equation . . . . . . . . . . . . . . . . 41

2.5 LTE vs. non-LTE . . . . . . . . . . . . . . . . . . . . . . 42

2.6 Inversion methods . . . . . . . . . . . . . . . . . . . . 44

3 Paper I - Spatially resolved water evolution 47

3.1 Summary . . . . . . . . . . . . . . . . . . . . . 47

3.2 Introduction . . . . . . . . . . . . . . . . . . . 48

3.3 MIRO observations . . . . . . . . . . . . . . . . . . . . . . . . . . . . . . . . . . . . . . . . . 49

3.4 Method . . . . . . . . . . . . . . . . . . . . 49

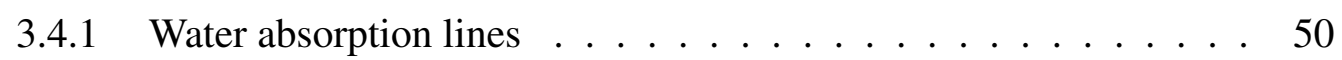

3.4 .2 Generating the lookup tables . . . . . . . . . . . . . . 51

3.4.3 Thermal sublimation model . . . . . . . . . . . . . 54

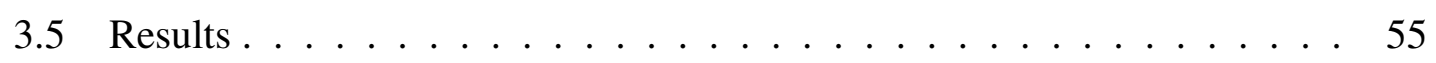

3.5 .1 Water production rate ........................ 55

3.5.2 Behaviour with heliocentric distance . . . . . . . . . . . . 58

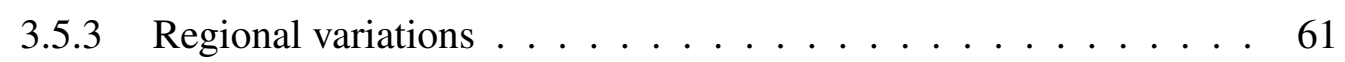

3.6 Conclusions . . . . . . . . . . . . . . . . . . 63 
4 Paper II - Interpretation of water production rates $\quad 67$

4.1 Summary . . . . . . . . . . . . . . . . . . . . 67

4.2 Introduction . . . . . . . . . . . . . . . . . . 68

4.3 Sublimation model . . . . . . . . . . . . . . . . . . 69

4.4 Results . . . . . . . . . . . . . . . . . . . 71

4.4.1 Effect of comet shape and obliquity . . . . . . . . . . 71

4.4.2 Effect of activity distributions . . . . . . . . . . . . . . 72

4.4 .3 Effects of obliquity and $\Phi \ldots \ldots \ldots 75$

4.5 Conclusions . . . . . . . . . . . . . . . 76

5 Paper III - Thermal inertia and roughness

5.1 Summary . . . . . . . . . . . . . . . . . . . 79

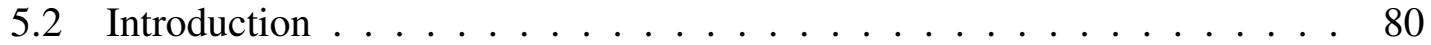

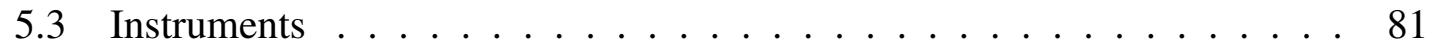

5.4 Methods . . . . . . . . . . . . . . . . . . . . 83

5.4 .1 Observational overlap . . . . . . . . . . . 83

5.4 .2 Thermal model . . . . . . . . . . . . . . . . . . 84

5.4.3 Radiative transfer model for MIRO data . . . . . . . . . . . . 85

5.4.4 Radiance model for VIRTIS data . . . . . . . . . . . . . . . 88

5.4 .5 Importance of roughness . . . . . . . . . . . . . . . . . . . . . . . . . . . . . . 99

5.5 Results . . . . . . . . . . . . . . . . . . . . 90

5.5.1 MIRO results . . . . . . . . . . . . . . . . . . 90

5.5.2 VIRTIS results . . . . . . . . . . . . . . . . 91

5.6 Discussion and conclusions . . . . . . . . . . . . . . . . 97

5.6.1 Thermal inertia . . . . . . . . . . . . . . . . . . 97

5.6 .2 Roughness . . . . . . . . . . . . . . . . . 99

6 Future work 101

6.1 Introduction . . . . . . . . . . . . . . . . 101

6.2 Method ............................. 102

6.2.1 Creating the a priori profiles . . . . . . . . . . . . 102

6.2 .2 Creating $K, S_{a}$ and $S_{e} \ldots \ldots \ldots \ldots$

6.2.3 Optimal estimation inversion . . . . . . . . . . . . 106

6.3 Application . . . . . . . . . . . . . . . . . 106

6.3.1 Synthetic case study . . . . . . . . . . . . . . . 107

6.3 .2 MIRO case study . . . . . . . . . . . . . . . . . . . . . . . . . . . . . . . . . . . . . . . . . .

6.4 Discussion . . . . . . . . . . . . . . . . . 111

7 Discussion $\quad 113$

$\begin{array}{ll}\text { Bibliography } & 117\end{array}$

A Derivation of radiative transfer equation 131

$\begin{array}{ll}\text { Publications } & 133\end{array}$

$\begin{array}{ll}\text { Acknowledgements } & 135\end{array}$ 



\section{Summary}

In August 2014, the Rosetta spacecraft completed its ten year journey when it arrived at its target destination, the comet 67P/Churyumov-Gerasimenko. The Rosetta mission was a flagship endeavour for the European Space Agency as it was the first time that any spacecraft had rendezvoused with a small solar system body for long period of time (two years) and also the first time that a lander had been placed onto the surface of a comet. In September 2016, the mission came to an end when Rosetta descended onto the surface for one final close up look at the surface.

This thesis uses data from one of the instruments on this ground-breaking mission: the Microwave Instrument for the Rosetta Orbiter (MIRO). MIRO was a spectrometer operating at millimetre and submillimetre frequencies and enabled the detection of several volatile species including water. Using the spectroscopic observations of the water lines, I investigated the behaviour of comet $67 \mathrm{P} /$ Churyumov-Gerasimenko relating to the gas coma, mass loss, spatial outgassing and the nucleus surface. Since comets are thought to be pristine building blocks left over from the formation of the solar system, we hope that by studying them, we can learn about the conditions from which other solar system bodies originate.

Firstly, I used the line areas of the $\mathrm{H}_{2}^{16} \mathrm{O}$ and $\mathrm{H}_{2}^{18} \mathrm{O}$ spectral lines to measure the change in the local water production rate from August 2014 to April 2016. Lookup tables made from a Haser model show how the measured Doppler shift velocity, the continuum temperature and the line area ratio can give the column density for each observation and thus the water production rate. The maximum production rate calculated from the MIRO observations was $(1.42 \pm 0.51) \times 10^{28} \mathrm{molec} / \mathrm{s}$, found on August 29, 2015, and integrating under all the data points gave a total water mass loss of $(2.4 \pm 1.1) \times 10^{9} \mathrm{~kg}$ for this apparition. By making assumptions about the dust-to-gas ratio and the comet mass, the total mass loss was estimated to be $(1.2 \pm 0.6) \times 10^{10} \mathrm{~kg}$, or $0.12 \pm 0.06 \%$. The spatial resolution of MIRO allowed for each measurement to be assigned to a region on the nucleus. The regions on the southern hemisphere appeared to be the origins of the highest production rates, in particular the regions Neith, Wosret and Bes. Finally, the data show that the production rate peak is offset by 22-46 days after perihelion and that the pre- and post-perihelion slopes followed power laws of $Q \propto r_{h}^{-3.8 \pm 0.2}$ and $Q \propto r_{h}^{-4.3 \pm 0.2}$, respectively.

Following this, I performed numerical modelling to investigate how nucleus shape, spin axis orientation and activity distribution affect the water production rate curves. I found that it is impossible to disentangle these effects from each other by only looking at the change in the production rate and that the pre- and post-perihelion slopes are also functions of heliocentric distance. It is therefore difficult to derive quantitative constraints on surface area ice fraction and active regions from the water production rate curve unless the shape, orientation and activity of the nucleus are well established. In addition, it may 
not be meaningful to compare the water production rate curves of different comets at different heliocentric distances.

I used the measured continuum temperatures from MIRO to derive properties of the nucleus in the third part of this work. I utilised an insolation driven thermal model to derive the temperature gradient in the upper layers of the comet surface and a radiative transfer model to reproduce the MIRO continuum measurements. In conclusion, a low value was derived for the thermal inertia in the surface layers of 67P with an upper bound estimated to be $80_{-40}^{+80} \mathrm{JK}^{-1} \mathrm{~m}^{-2} \mathrm{~s}^{-0.5}$ for most of the MIRO measurements. A low value for the average thermal inertia over the entire surface would be consistent with the majority of reported calculated values for 67P.

In the future, the retrieval of coma parameters from the MIRO spectra will become an important avenue of investigation. Using inverse methods, the behaviour of the gas temperature, expansion velocity and molecular number density profiles can be extracted from the spectral lines. This will be important for assessing and characterising the activity around the nucleus which is observed but not so well understood. In addition, we may learn more about the physics of the coma from the inversion solutions, such as the presence of a Knudsen layer, photolytic heating or extended sources. At the end of my thesis, the application of inverse methods to the MIRO data is described.

It is still uncertain to what extent 67P is pristine and primordial but the results of my thesis imply that the surface layer over a couple of metres must be processed by the sun during perihelion passage. In this region, icy volatiles sublimate as the thermal wave propagates into the surface, expelling dust and refractory material away from the nucleus along with the gas. In the most productive regions on the south, the erosion is expected to reach down to a few metres in depth. The proposed CEASAR sample return mission would revisit $67 \mathrm{P}$ and the work for my thesis suggests that the drill must go beyond the surface layer, perhaps as much as 10 times the diurnal skin depth, in order to reach any potentially pristine material. 


\section{Zusammenfassung}

Im August 2014 hat die Raumsonde Rosetta ihre zehnjährige Reise beendet, als sie am Ziel, dem Kometen 67P/Churyumov-Gerasimenko angekommen ist. Die Rosetta-Mission war eine Flaggschiff-Mission der europäischen Weltraumagentur, da die Raumsonde zum ersten Mal mit einem kleinem Körper im Sonnensystem für einen längeren Zeitraum (zwei Jahre) in Kontakt war und eine Landeeinheit zum ersten Mal auf einer Kometenoberfläche platziert wurde. Als Rosetta im September 2016 für einen letzten detaillierten Blick zur Kometenoberfläche herunter gefahren ist, fand die Mission ihr Ende.

Diese Doktorarbeit verwendet Daten eines der Instrumente dieser bahnbrechenden Mission: das Mikrowellen-Instrument für den Rosetta Orbiter (MIRO). MIRO war ein Spektrometer, welches im Millimeter- und Submillimeter-Frequenzbereich arbeitete und die Entdeckung mehreren flüchtigen Spezies einschließlich Wasser ermöglicht hat. Bei der Anwendung der spektroskopischen Beobachtungen der Wasserlinien habe ich das Verhalten des Kometens 67P/Churyumov-Gerasimenko in Bezug auf die Koma, den Massenverlust, räumliche Ausgasungen und die Kernoberfläche untersucht. Da Kometen als vormalige Bausteine aus der Zeit der Entstehung des Sonnensystems angesehen werden, erhoffen wir, dass wir bei deren Untersuchung über die Entstehungsbedingungen anderer Körper im Sonnensystem lernen können.

Zuerst habe ich die Linienintegrale der Spektralinien $\mathrm{H}_{2}^{16} \mathrm{O}$ zur Messung der Veränderung der lokalen Wasserproduktionsrate zwischen August 2014 und August 2016 untersucht. Mit Hilfe von Look-Up Tabellen aus dem Haser Model kann von den gemessenen Doppler-Geschwindigkeiten, der Kontinuums-Temperatur und dem Verhältnis der Linienintegrale auf die Säulendichte und somit die Wasserproduktionsrate geschlossen werden. Die von den MIRO-Beobachtungen gerechnete höchste Wasserproduktionsrate war $(1.42 \pm 0.51) \times 10^{28} \mathrm{molec} / \mathrm{s}$, die am 29. August 2015 gefunden wurde. Beim Intergrieren aller Datenpunkte hat sich für dieses Ereignis ein Gesamtwasserverlust von $(2.4 \pm$ 1.1) $\times 10^{9} \mathrm{~kg}$ ergeben. Mit Hilfe von Annahmen über das Staub-Gas-Verhältnis und der Kometenmasse, wird der Gesamtverlsut auf $(1.2 \pm 0.6) \times 10^{10} \mathrm{~kg}$ oder $0.12 \pm 0.06 \%$ der Kometenmasse geschätzt. Dabei hat es die räumliche Auflösung von MIRO ermöglicht, dass jeder Messung eine Region am Kometenkern zugewiesen werden konnte. Die Regionen an der Südhalbkügel, insbesondere die Regionen Neith, Wosret und Bes erschienen als die Ursprünge der höchsten Produktionsraten. Letztendlich zeigen die Daten, dass die maximale Produktionsrate um 22 bis 46 Tage zum Perihelion versetzt ist und die Produktionsraten vor und nach dem Perihelion den Potenzgesetzen $Q \propto r_{h}^{-3.8 \pm 0.2} \mathrm{bzw}$. $Q \propto r_{h}^{-4.3 \pm 0.2}$ folgen.

Im Anschluss daran habe ich numerische Modellierungen durchgeführt, um zu untersuchen, wie die Kometenform, die Ausrichtung der Rotationsachse und die Aktivitätsverteilung die Wasserproduktionsrate prägen. Ich habe festgestellt, dass nicht möglich 
ist diese Effekte getrennt voneinander zu betrachten, wenn lediglich die Veränderung der Produktionsrate betrachtet wird. Zudem sind die Produktionsraten vor und nach Perihelion abhängig von der heliozentrischen Distanz. Deswegen ist es schwierig, die quantitativen Beschränkungen auf der Oberfläche der eisbedeckten Gebiete und der aktiven Regionen von der Wasserproduktionsratekurve herzuleiten, außer wenn die Kernform, die Kernausrichtung und die Kernaktivität gut bestimmt sind. Zudem könnte es nicht sinnvoll sein, die Wasserproduktionsratenkurven verschiedender Kometen bei verschiedenen heliozentrischen Distanzen zu vergleichen.

Im dritten Teil dieser Arbeit habe ich die gemessene Kontinuumstemperatur von MIRO zur Herleitung der Kerneigenschaften verwendet. Ich habe ein von Einstrahlung reguliertes thermisches Modell zur Derivation das Temperatursgradienten in den oberen Schichten der Kometenoberfläche verwendet, sowie ein Strahlungstransportmodell zur Wiederherstellung der MIRO-Kontinuumsmessungen. Damit wurde ein niedriger Wert für die thermische Trägheit in den Oberflächenschichten von 67P gefunden. Der Wert hatte eine geschätzte obere Grenze von $80_{-40}^{+80} \mathrm{JK}^{-1} \mathrm{~m}^{-2} \mathrm{~s}^{-0.5}$ für die Mehrheit der MIROMessungen. Ein niedriger Wert für die durchschnittliche thermische Trägheit über die Gesamte Oberfläche, würde konsistent mit dem Großteil der für 67P errechneten Werte sein.

In der Zukunft wird die Gewinnung von Kometenparametern von den MIRO-Spektren eine wichtige Untersuchungsrichtung. Bei der Verwendung von inversen Methoden können das Verhalten der Gastemperatur, der Ausbreitungsgeschwindigkeit und der Molekülanzahldichteprofile von den Spektrallinien extrahiert werden. Dieses Ergebnis wird wichtig für die Beurteilung und Charakterisierung der Aktivität um den Kern sein, die beobachtet wird, aber noch nicht gut verstanden ist. Zusätzlich können wir durch Inversionslösungen unser Wissen der Physik der Koma erweitern, wie die Gegenwart einer Knudsen-Schicht, photolytische Erwärmung oder erweiterte Quellen. Am Ende meiner Doktorarbeit wird die Anwendung inverser Methoden auf die MIRO-Daten beschrieben.

Es ist immer noch unklar, inwieweit 67P unberührt und unberührt ist, aber die Ergebnisse meiner Arbeit deuten an, dass die Oberflächenschicht über eine Dicke von wenigen Metern während des Periheldurchgangs durch die Sonne bearbeitet wurde. In dieser Region sublimieren Eisvolatile während die thermische Welle sich in die Oberflächenschichten ausbreitet, wobei sie Staub und hitzebständiges Material, sowie das Gas vom Kern ausgestoßen werden. In den hochaktiven Region im Süden ist die Erosion voraussichtlich ein paar Meter tief. Die vorgeschlagene CEASAR-Mission, die eine Probe liefern soll, würde 67P wieder besuchen. Meine Arbeit weist darauf hin, dass das Bohrgerät zur Erreichung eventuell unberührtes Materials durch die Oberflächenschicht weiter hinaus, vielleicht so tief wie zehnmal der tageszyklischen Eindringtiefe, bohren muss. 


\section{Introduction}

\subsection{Comets}

Comets are small solar system bodies made from icy volatile and rocky refractory material. They are typically a few kilometres in size $(1-10 \mathrm{~km})$, with the Great Comet of 1577 (C/1577 V1) and Comet Sarabat of 1729 (C/1729 P1) thought to be the largest with diameters from 100-300 km (Clube et al. 1996), whereas the bi-lobed comet 103P/Hartley has a maximum length measuring only $2.33 \mathrm{~km}$ (A'Hearn et al. 2011). Due to their small size, comets are usually invisible when they are far away from the Sun and appear as only point sources, indistinguishable from the background stars. However, when they come close to the sun, comets become much brighter and develop observable comae and tails, as shown in Figure 1.1. The volatile content within the nucleus sublimates as a result of the increase in received solar flux as the heliocentric distance decreases and the sublimated gas forms a coma, a tenuous atmosphere around the comet. The coma also contains dust grains which are lifted from the nucleus by the outflowing gas, although this historical idea seems to be somewhat more complicated as the exact mechanism of lifting dust is unknown - gas drag appears to be insufficient to break the cohesion force between grains on the surface (Skorov et al. 2017). The size of the coma depends upon how it is defined (Rodgers et al. 2004): the collisional region may be $10-100 \mathrm{~km}$ in size and the $\mathrm{H}_{2} \mathrm{O}$ coma can be $10^{5} \mathrm{~km}$ in radius, whilst the atomic hydrogen coma can measure as much as $10^{7} \mathrm{~km}$. However, the material in the coma is not gravitationally bound due to the small size of comets. An ion tail grows as ionised gas and fine grained dust is pushed away from the nucleus, and a dust tail forms from solar radiation pressure. Both tails always point away from the sun, but the ion tail is always in a radial direction from the sun due to interactions with the solar wind, and the dust tail is curved, as the small dust grains start to move on their own Keplerian orbits (Karttunen et al. 2016). The tails and the coma become optically observable due to the dust material within them reflecting light.

Comets are almost ubiquitous throughout the solar system, with some comets having orbits which keep them close to the terrestrial and giant planets, others on long period orbits beyond Neptune ( $>30 \mathrm{AU}$ ) which take them through the scattered disk and the Kuiper Belt, and some located in the furthest reaches of the Oort Cloud (>1000 AU), the spherical shell that encompasses the solar system. However, the most distant observation of a comet was of Hale-Bopp at 32 AU (Szabó et al. 2012) and due to their dimness, it is difficult to observe any comets beyond that in the scattered disk or the Oort Cloud. The evidence for comets in these regions comes from the reconstructed orbits of comets which enter the inner solar system. The most distant comets are the nearly isotropic comets (NICs) which have semi-major axes from $~ 40$ AU to over 10,000 AU and they 


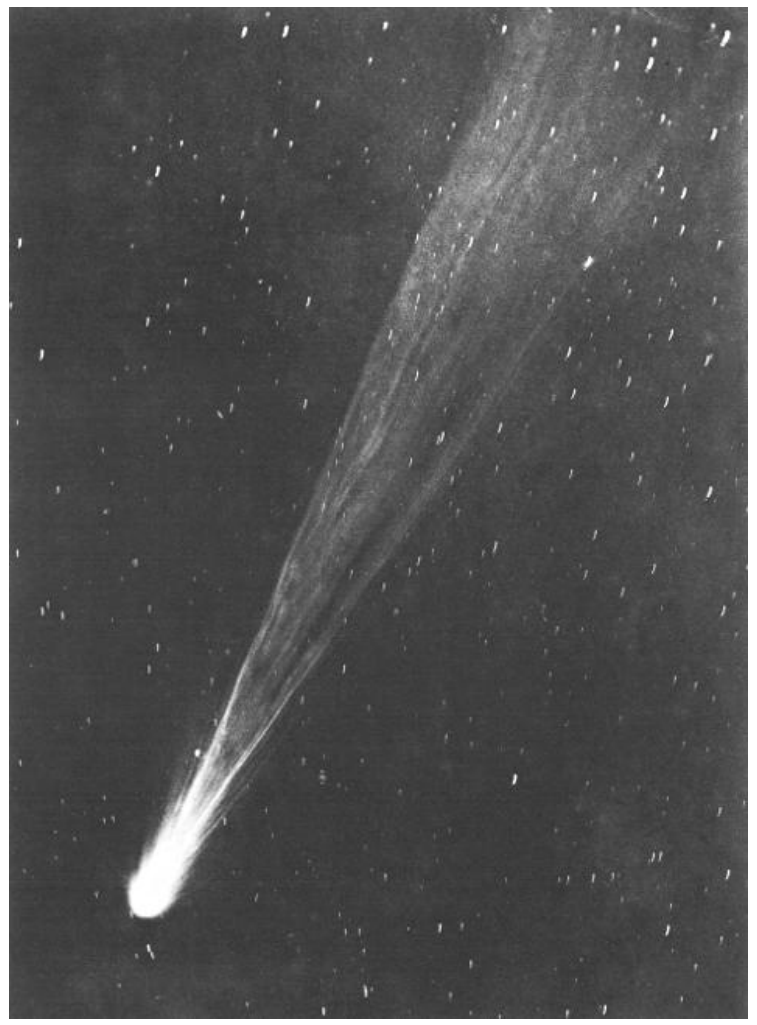

Figure 1.1: C/1911 O1 (Brooks), discovered by William Robert Brooks. (Credit: Paul Anderson, 1911) The image shows the large comet tail forming behind the nucleus.

reside beyond Neptune, in the scattered disk, the Kuiper Belt and the Oort Cloud (Levison 1996, Levison and Dones 2014). There are three types of NICs, new comets, which are the furthest away, returning comets, and Halley-type comets. Ecliptic comets are found in the orbital plane and have semi-major axes less than $40 \mathrm{AU}$, bringing them under the gravitational influence of the planets. One particular class of ecliptic comets are called Jupiter-Family Comets. These comets cross the orbit of Jupiter and feel its strong gravitational attraction (Duncan et al. 2004, Dones et al. 2004).

The dynamical lifetimes of comets are short compared to the age of the solar system and so they must have been put on their current orbits relatively recently and come from from some other reservoir (Duncan et al. 2004). Two pieces of observational evidence suggest that NICs and the ecliptic comets originate from different reservoirs (Levison and Dones 2014). The first is that while some comets change minor class (i.e. Jupiter-Family comet to Chiron-type, or new to returning comet), comets do not generally change major class (NIC to ecliptic) (Levison 1996). In addition, the inclination distribution for NICs is very isotropic, suggesting they come from all directions (hence the name for this class) (Dones et al. 2004), but the inclination distribution for ecliptic comets is much narrower suggesting they come from a different region (Levison and Dones 2014). Even Halleytype comets have a more confined range of inclinations than new and returning comets. It is generally thought then that NICs come from from the Oort Cloud at the edge of the solar system, coming into the inner solar system after gravitational perturbations from outside influences; and that ecliptic comets have origins in the scattered disk, a region 
that extends from Neptune to the inner edge of the Oort Cloud, where numerous transNeptunian objects have been observed. Halley-type comets appear to originate from an intermediate region where the spherical Oort cloud is becoming more disk-like.

However, it is also thought that comets did not initially form in these reservoirs, and that they have a complex dynamical history due to the supposed migration of the planets during the early formation of the solar system. It is thought that most comets should have formed around and beyond the snow line where the giant planets are currently located (Dones et al. 2004, 2015). In the early evolution of the solar system, as the swirling disk of gas rotated around the proto-Sun, small planetesimals started to form and grow in size. Some of these objects coalesced to form the planets whereas others where slowly kicked out of the inner solar system by planetary interactions, increasing their semi major axis and perihelion distance. These planetesimals which were kicked out into the scattered disk and the Oort Cloud are the comets which we can now see today.

Through spectroscopic observations and satellite flybys, the composition of comet nuclei is slowly being revealed. In 1950, a revolutionary time for cometary science, it was first proposed that comets are dirty snowballs (Whipple 1950) made from volatile ices and dusty refractory material. Our knowledge of the volatile component arises mainly from spectroscopy, which has allowed dozens of molecules to be identified in the coma of comets (Bockelée-Morvan 2011). The main component is water, accounting for up to $80 \%$ of the volatile content. The abundance of other species are usually given as relative to the water content. After water, $\mathrm{CO}$ and $\mathrm{CO}_{2}$ are two of the more abundant molecules observed in cometary comae but the abundances in the coma vary from comet to comet. For example, in most comets so far observed, $\mathrm{CO}_{2}$ dominates $\mathrm{CO}$, however for comet $\mathrm{C} / 2006 \mathrm{~W} 3$ (Christensen), the situation appears to be reversed and $\mathrm{CO}$ dominates $\mathrm{CO}_{2}$ (Ootsubo et al. 2012). Deriving classification based on the ratios of volatile abundances is very difficult though due to the high variability in the abundances, for example, the $\mathrm{CO}_{2} / \mathrm{H}_{2} \mathrm{O}$ ratio can vary with heliocentric distance and with latitude on the surface (Mall et al. 2016). There is also further evidence that the molecular abundances in the coma are not constant as seen with comet $67 \mathrm{P} /$ Churyumov-Gerasimenko, where the number density ratio of $\mathrm{CO}_{2} / \mathrm{H}_{2} \mathrm{O}$ has been observed to vary between $2.5 \%$ and $80 \%$ (Fougere et al. 2016) as the heliocentric distance changes and as observations are made on different parts of the nucleus. Abundances can be measured for a single observation but this is only a single snapshot of the overall complicated outgassing behaviour. Volatile molecules have different sublimation temperatures and so the outgassing of certain ices switches on at different heliocentric distances. All of this must be considered when interpreting volatile abundance ratios.

Other molecules observed in relatively high abundances include $\mathrm{CH}_{3} \mathrm{OH}, \mathrm{CH}_{4}, \mathrm{H}_{2} \mathrm{~S}$ and $\mathrm{NH}_{3}$. These species, in addition to $\mathrm{CO}$ and $\mathrm{CO}_{2}$, are usually observed to have abundances of $0.01 \%-20 \%$ relative to water. Figure 1.2 lists a number of observed volatile species and their relative abundances to water.

There are ongoing questions as to whether the spectroscopic observations made of the coma are representative of the nucleus, and even how much the nucleus may have evolved since its formation. Comets may experience some evolution far away from the sun in the Oort Cloud as well as during close perihelion passages when the volatile material sublimates (Bockelée-Morvan 2011). Evidence from 73P/Schwassmann-Wachmann 3 , which started to disintegrate as it entered the inner solar system, shows that the dif- 


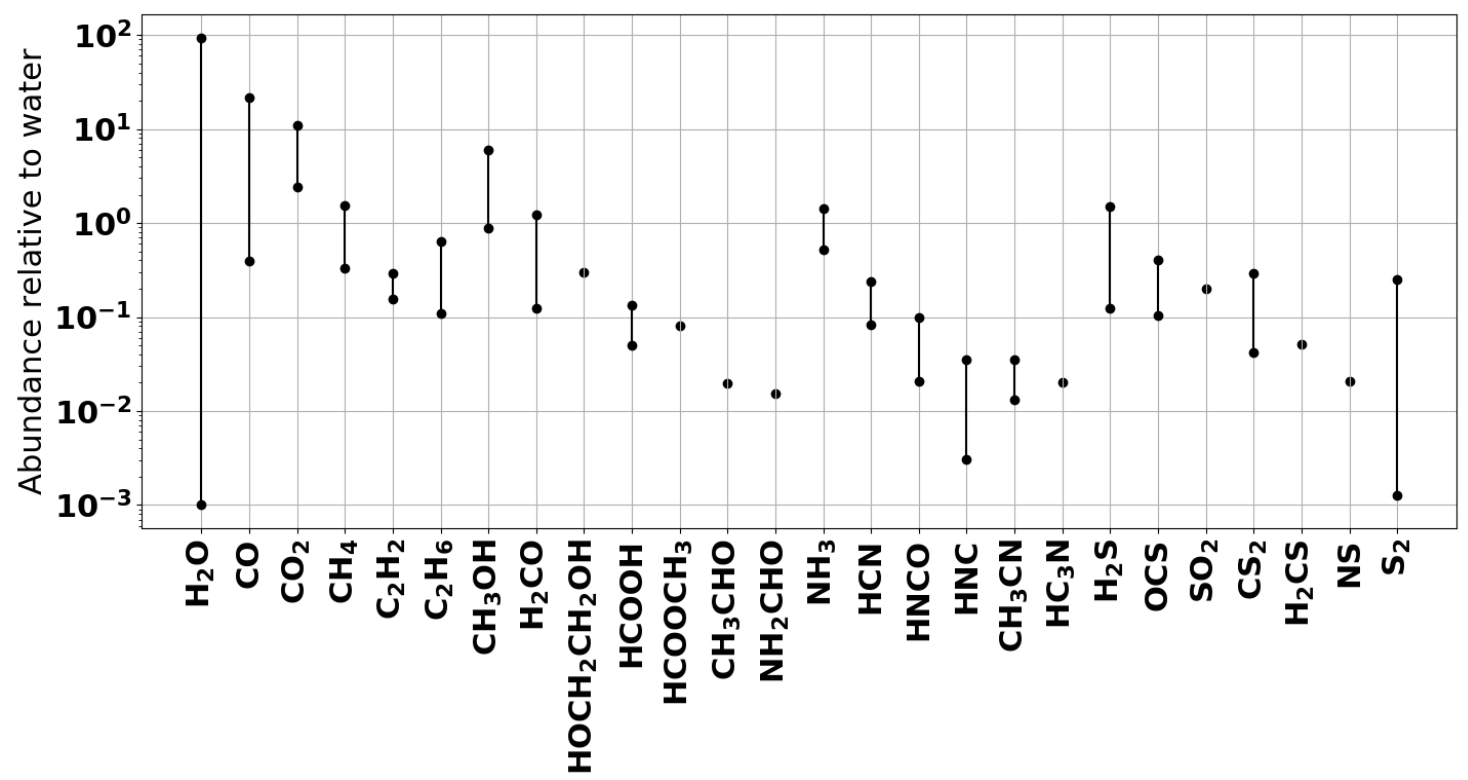

Figure 1.2: Abundance ratios relative to water for a variety of molecules observed in cometary comae (based on Fig. 1 of (Bockelée-Morvan 2011)).

ferent cometary fragments have similar chemical abundances, suggesting that chemical abundances could be primordial signatures of formation rather than from evolutionary processes over the comets lifetime (Cochran et al. 2015).

Information on the refractory part of comets mainly arises from cometary space missions (Bockelée-Morvan 2011). The dust collected from around comet 1P/Halley was analysed using mass spectrometry to determine the composition. Elements such as $\mathrm{Mg}$, $\mathrm{Si}, \mathrm{Fe}$ and $\mathrm{Ca}$ were found, as well as organic material including $\mathrm{CHON}$ grains.

The study of comets is thought to be very important as they have profound implications for our solar system. Far from the sun where the temperatures are low, comets should remain relatively unprocessed over their lifetimes and the interior material must be relatively pristine and primordial. If comets do originate from the very early formation of the solar system and were ejected to the furthest reaches of the sun, then they may hold clues to the conditions and evolution history of our solar system and could shed light on what was happening approximately 4.5 billions of years ago.

\subsection{Observational history: from the stone age to the space age}

Remarkably, it is argued that the earliest recorded sightings of comets come from the latter half of the Shang (or Yin) Dynasty which ruled in Ancient China between 1500 $1050 \mathrm{BC}$. The observations were recorded on animal bones and turtle shells and they are known as the Oracle Bones, containing records of solar eclipses, lunar eclipses, planets and comets (Zhen-Tao et al. 1995). A few hundred years later, the Babylonians also recorded observations of comets in their Astronomical Diaries, which covered the period 
from $750 \mathrm{BC}-75 \mathrm{AD}$, and wondered about their origins, speculating that these remarkable objects in the sky were obscure planets (Stephenson et al. 1985). The Babylonian records also include mentions of probable observations of comet 1P/Halley in $164 \mathrm{BC}$ and $87 \mathrm{BC}$. Ephorus, the Greek historian, believed that comets were the result of collisions between stars after observing the Great Comet of $371 \mathrm{BC}$, which is now suspected to have been a Sun-grazing comet that broke apart during this apparition (Seargent 1982).

However, without a proper explanation, other people believed that, rather than being astronomical bodies, comets were actually meteorological phenomenon (Seargent 1982). Numerous famous figures throughout history, including Pliny the Elder, Ptolemy and Galileo, thought that comets were atmospheric manifestations like reflections of the Sun or features from volcanic eruptions.

In addition, comets were also mistakenly regarded as portents of doom by some people, heralding a natural disaster, the death of a monarch or attacks by heavenly forces. Across Europe, the Middle East and Asia, the appearance of a comet was treated with suspicion and superstition. The $164 \mathrm{BC}$ apparition of 1P/Halley coincided with the death of Antiochus IV, the ruler of the Selucid Empire, as well as the expulsion of the Ptolemaic ruler Philometor from his throne (incidentally, both of their successors were replaced within two years) (Wolters 1993). In 44 BC, the apparition of a comet coincided with the death of Julius Caesar, as written about by William Shakespeare (Seargent 1982). The Bayueux Tapestry, a medieval piece of artwork depicting the successful conquer of England by William the King of Normandy, also envisions a comet in the background, an omen of the end of the reign of the English King Harald. Giotto di Bondone, the $14^{\text {th }}$ century painter, was so taken by the mystical elements of comets that he chose to include a comet in his painting, The Adoration of the Magi, showing the birth of Jesus Christ in a nativity scene (Crovisier et al. 2000).

It was not until Tycho Brahe made astronomical observations of the solar system during the $16^{\text {th }}$ Century that the idea of comets as astronomical bodies returned to prominence (Seargent 1982). Brahe made meticulous measurements of the Great Comet of 1577 from his observatory in Hven, Sweden, in conjunction with Thaddeus Hagecius in Prague, and determined the parallax for the comet (Christianson and Brahe 1979). In their observations, the comet was approximately in the same position on the sky for both of them but the Moon was not, leading Brahe to conclude that the comet must be further away from Earth than the Moon and therefore not an atmospheric phenomenon. A student of Brahe, Johannes Kepler, confirmed this finding by measuring the parallax of the comets of 1607 and 1618 (Seargent 1982).

The exact nature of comets was still unknown in this time but Edmond Halley shed some more light on the nature of cometary objects when he observed comets in 1680 and 1682 (Seargent 1982). After calculating the orbit in collaboration with Isaac Newton (Gribbin 2004), he speculated that the second one may have been the same comet which had appeared in 1607 and 1531, and that comets had a periodic nature. He predicted that the comet would return in 1758. When the comet reappeared, Halley's prediction proved to be correct, although he did not live to see this as he died in 1742, sixteen years before the apparition. In addition, Halley thought, much like Kepler before him, that the comets which they had seen were only one of many in existence.

It was not until the year 1950 that cometary science took a huge leap forward with the publication of three seminal papers which underpin our basic understanding of comets 
(Festou et al. 2004). The first was the "dirty snowball" view of comets proposed by Fred Whipple (Whipple 1950), which postulated that comets were icy conglomerates containing a mixture of volatile material that sublimated in increasing quantities as the comet approached the sun and releasing dust grains from the surface. It was an important idea which encompassed many of the observed aspects of comets which had been documented in earlier work (Festou et al. 2004), such as: the presence of molecular daughter species $\left(\mathrm{CO}^{+}, \mathrm{CN}\right)$ from more stable ices $\left(\mathrm{CO}, \mathrm{CO}_{2}\right)$; the large production rates of comets; jet-like structures; the influence of nongravitational forces on their motion; and meteor streams. The work of Whipple (1950) was widely accepted at the time and it is still of major importance today.

The second novel idea was Jan Oort's idea (Oort et al. 1950) of a halo surrounding the solar system containing a huge number of comets: the Oort Cloud. This idea evolved from the distribution of the semi major axis of comets. Over time our understanding of the Oort Cloud has improved but the work of Oort has informed our current understanding and helped define the taxonomic classes described in Section 1.1.

The connection between the comet tail and the solar wind was the third finding in this time frame from Biermann (1951). It was already suspected that magnetic activity on Earth was influenced by solar flares (Festou et al. 2004), but it was Biermann's work which hypothesised that the ions in a cometary tails were interacting with electrically charged particles of the solar wind.

Clearly, our knowledge of comets has improved over the centuries, and we now know that comets are not meteorological or prescient objects at all, but bodies in our solar system. With the advent of space exploration in the latter half of the $20^{\text {th }}$ century, it became possible to fly instruments to distant comets and study them close up for the very first time.

Studying objects deep in the Oort Cloud, Kuiper Belt or the scattered disk is not easy though, and going to them for close up exploration is almost impossible. If we want to study comets, we must then look a little closer to home. The Jupiter Family Comets, although processed by their close approaches to the Sun, may still contain some of their pristine nature and by studying them in great detail, we can try to answer one of the greatest mysteries in space science: how the solar system came to be.

The history of cometary spacecraft exploration is only 33 years old and in total, only eight different comets have been visited by ten different spacecraft (Schulz 2009). The first was the NASA spacecraft, the International Cometary Explorer (ICE), which visited comet 21P/Giacobini-Zinner in 1985 and made measurements of particles, waves and fields. ICE probed the interaction between the comet and the solar wind, confirming the Alfveń model structure of a comet tail (two lobes of opposite polarity magnetic field lines separated by a current sheet), detecting water and carbon monoxide ions with the water group ions dominating, and measuring collisions of dust particles with the spacecraft (Von Rosenvinge et al. 1986).

A year later, a collection of five spacecraft, unofficially known as the Halley Armada, visited comet 1P/Halley during its apparition in 1986. These were: the Russian probes, VeGa 1 and VeGa 2; Sakigake and Suisei, built by the Institute of Space and Astronautical Science (ISAS, now known as the Japanese Aerospance Exploration Agency, JAXA); and Giotto from the European Space Agency (ESA) (Festou et al. 2004). VeGa 1 sent back the first images of a comet nucleus (Sagdeev et al. 1986) and measured the temperature of 


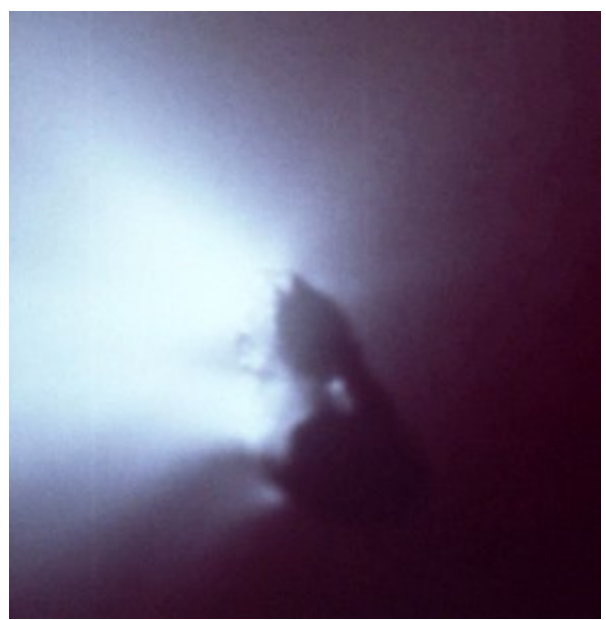

(a)

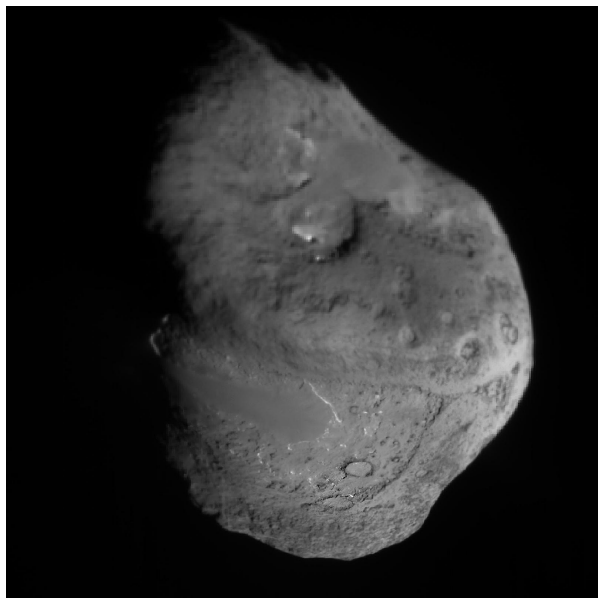

(c)

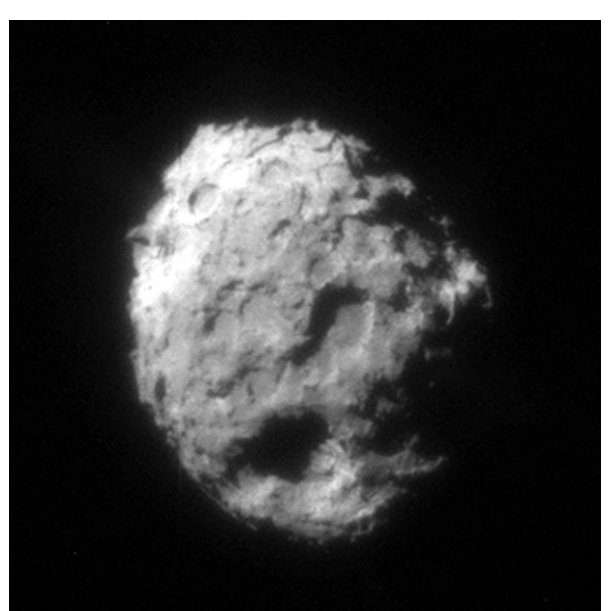

(b)

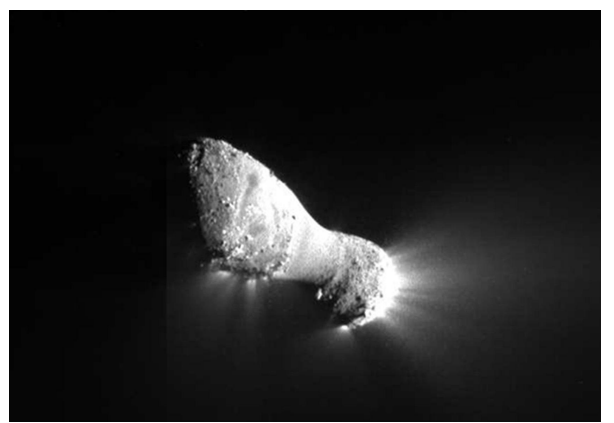

(d)

Figure 1.3: Spacecraft images of comets. (1.3a) the nucleus of 1P/Halley as seen by the ESA Giotto probe in 1986 (Credit: ESA, 2000-2008 (C) European Space Agency), (1.3b) the nucleus of 81P/Wild, taken by the Stardust mission in 2004 (Credit: Courtesy NASA/JPL-Caltech.), (1.3c) the nucleus of 9P/Tempel, seen from the Deep Impact mission in 2005 (Credit: Courtesy NASA/JPL-Caltech.), (1.3d) the nucleus of 103P/Hartley as seen by EPOXI in 2011 (Credit: Courtesy NASA/JPL-Caltech.) 
the surface to be 300-400 K, much warmer than expected for an icy body (Combes et al. 1986, Emerich et al. 1988). This implied there must be a layer of dust material at the surface. VeGa 2 made a closer approach to the surface and enabled the determination of the physical size of the nucleus (Sagdeev et al. 1986). Suisei took measurements of 1P/Halley with an ultraviolet imager and confirmed the hard surface of the nucleus, as well as determining some solar wind parameters (Kaneda et al. 1986). Observations from Sakigake lead to the proposal of an Interplanetary Magnetic Field-comet interaction model (Saito et al. 1986). Finally, Giotto encountered Halley, getting even closer to the nucleus, and became the first instrument to make mutlicolour images of a comet (Thomas and Keller 1988), see Figure 1.3a. This revealed the jet-like structures made from fine dust as well as morphological features (Schwarz et al. 1988). Giotto also helped to constrain predominance of water in the coma (Krankowsky et al. 1986), and the size and composition of the ejected dusty material, including the presence of CHON elements (Clark et al. 1988).

In 1992, the Giotto Extended Mission visited the comet 26P/Grigg-Skjellerup, making the closest approach of any comet until the 2014 Rosetta mission (Schulz 2009). Despite the failure of the camera, which was damaged and inoperable after its encounter with Halley, it was found that comet 26P/Grigg-Skjellerup is much smaller than 1P/Halley and relatively inactive in comparison.

Fifteen years passed before the next cometary mission when Deep Space 1 travelled to comet 19P/Borrelly. Although the main goal of the mission was a flyby of asteroid Baille, the Deep Space encounter with Borrelly was incredibly successful, making images of a Jupiter Family Comet for the first time, and at a higher resolution than ever before (Boice et al. 2000). The images revealed an exceptionally dark surface, with albedo variations from $0.007-0.035$, and a hot, dry surface with a sublimating area less than $10 \%$ of the whole nucleus. The PEPE plasma instrument also observed inhomogeneities in the plasma data around the comet (Richter et al. 2011).

Comet 81P/Wild was the subject of the first ever comet sample return mission, with the Stardust spacecraft making a rendezvous with the comet in 2004, before completing its mission in January 2006 when the samples reached Earth (Brownlee 2014). Stardust contained several deposits of low density silica aerogel to collect dust particles ejected from the surface of the comet and stored them for return and analysis in a laboratory. The returned particles shed a lot of light on the early history of the Solar System. The dust component was similar in material to meteorites and contained a low abundance of material from the presolar nebula. This implies that $81 \mathrm{P} /$ Wild formed during the very early stage of the solar system and from material which condensed at different heliocentric distances, from the hot interior where the rocky components condensed to the cold outer regions where the supervolatile material formed. The images from Stardust showed $81 \mathrm{P} /$ Wild to be a $5 \mathrm{~km}$, round body, with many ancient surface features despite its active nature (Brownlee et al. 2004), as shown in Figure 1.3b.

The next cometary mission was the visit of Deep Impact to 9P/Tempel in 2005. The unique aim of this mission was to study the interior composition of a comet by firing an impactor, a $360 \mathrm{~kg}$ projectile, at the nucleus and thus analyse the resulting exposed debris for pristine material (Hampton et al. 2005). The observations from Deep Impact revealed several things about the comet (A'Hearn et al. 2005): the nucleus has a density of approximately $600 \mathrm{~kg} \mathrm{~m}^{-3}$ and a diameter of about $3 \mathrm{~km}$; there was an observed increase in organic material relative to water during and after the impact; the upper layer of nucleus 
is made of fine particles $(1-100 \mu \mathrm{m})$ with negligible strength and these particles may be present to decimetre scale depths; the surface is in equilibrium with sunlight; and ice does not appear to be on the surface so the observed outgassing must come from volatile material near the surface. A Stardust image of 9P/Tempel is shown in Figure 1.3c

Renamed EPOXI, the Deep Impact spacecraft was requisitioned for two further missions. The first was to perform a fly-by of another comet, and the second aim was to use its imaging cameras as a space observatory to observe exoplanets and comets. In November 2010, EPOXI made a close fly-by of comet 103P/Hartley, a very active comet despite being quite small, depicted in Figure 1.3d. In fact, the comet was producing more water than would be possible from an object of its size (A'Hearn et al. 2011). $\mathrm{CO}_{2}$ must therefore have been an important driver of activity, releasing icy dust grains from the surface to enhance the water production. From the images, it appeared as if most of the water outgassing was coming from the waist of the elongated comet. During the observatory phase, EPOXI made observations of Garradd (C/2009 P1) (Feaga et al. 2013) and C/2012 S1 (ISON) (Farnham et al. 2017), measuring the volatile production rate of these comets. The mission terminated in 2013 after contact with the spacecraft was lost.

The Stardust mission was extended (called Stardust-NExT) and made a flyby of 9P/Tempel in 2011, enabling direct comparisons of observations from Deep Impact (Veverka et al. 2013). The additional observations from Stardust-NExT enabled the coverage of the nucleus to be extended to two-thirds of the surface in combination with the Deep Impact images. The surface showed evidence of layering, deep pits in the nucleus, and some changes in small regions since the 2005 fly-by, although the majority of the surface was relatively unchanged. The jet activity also appeared lower than before.

The most recent exploration of a comet occurred only three years later with the arrival of the Rosetta spacecraft at the comet 67P/Churyumov-Gerasimenko.

\subsection{The Rosetta mission}

The Rosetta mission was first envisaged in 1991 as a sample return mission as part of the European Space Agency Horizon 2000 programme (Huber and Schwehm 1991). ESA approved the Rosetta mission in 1993. The sample return aspect of the mission was later dropped but many of the key aims remained unchanged: to probe the origins of cometary formation and evolution, as well as to learn about the origins of the solar system (Glassmeier et al. 2007). As has been noted before, comets are thought to contain some of the most pristine material from the early solar system, and through the study of comets, we can try to determine the history and development of the solar system.

In the beginning, the expected target for the mission was comet 46P/Wirtanen (Schwehm and Schulz 1999). Comet 46P/Wirtanen is thought to be a small but highly active comet, with layers of exposed material on its surface as a result of the complete removal of material from the strong outgassing (Groussin and Lamy 2003). However, due to the failure of an Ariane rocket before take off, the original launch date in 2003 was delayed until March 2004 (Glassmeier et al. 2007). Consequently, the delay meant that 46P/Wirtanen was no longer on a favourable orbit and a new target comet had to be

found. In this case, comet $67 \mathrm{P} /$ Churuyumov-Gerasimenko was chosen, and this comet is discussed in the next section. 
Despite the change in target, the goals of the Rosetta mission remained unchanged since the initial proposal, and are described in Schwehm and Schulz (1999), Glassmeier et al. (2007) and Schulz (2009). The mission aimed to:

- globally characterise the comet in terms of dynamic properties, surface morphology and composition

- determine the chemical, isotopic and mineralogical composition of volatile and refractory material in the nucleus

- determine the physical properties of the volatile and refractory material, and their interrelations

- probe the development of cometary activity and surface processes in the nucleus and the inner coma

- study the evolution of outgassing and solar wind interactions during perihelion voyage

- characterise main belt asteroids in terms of dynamic properties, surface morphology and composition

The Rosetta spacecraft was finally launched on March 2, 2004 from Kourou, French Guiana, and consisted of an orbiter, Rosetta, and a lander, Philae. The orbiter contained eleven instruments (Glassmeier et al. 2007), as shown in Figure 1.4, including:

- Alice (ultraviolet imaging spectrometer)

- CONSERT (COmet Nucleus Sounding Experiment by Radio wave Transmission)

- COSIMA (COmetary Secondary Ion Mass Analyser)

- GIADA (Grain Impact Analyser and Dust Accumulator)

- MIDAS (Micro Imagaing Dust Analysis System)

- MIRO (Microwave Instrument for the Rosetta Orbiter)

- OSIRIS (Optical, Spectroscopic and Infrared Remote Imaging System)

- ROSINA (Rosetta Orbiter Spectrometer for Ion and Neutral Analysis)

- RPC (Rosetta Plasma Consortium)

- RSI (Radio Science Investigation)

- VIRTIS (Visible and InfraRed Thermal Imaging Spectrometer)

In addition, the Philae lander housed a further ten instruments:

- APXS (Alpha Proton X-ray Spectrometer) 


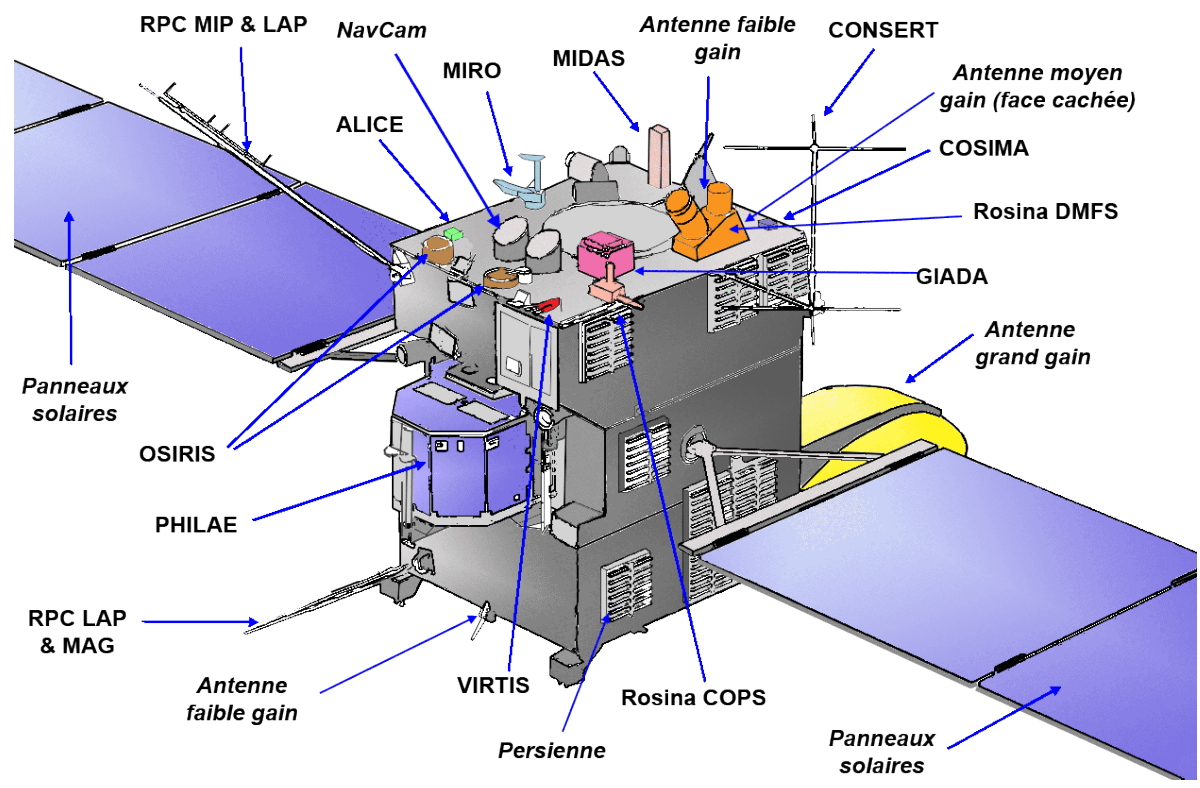

Figure 1.4: Schematic diagram of the Rosetta orbiter with instruments labelled (Credit: Pline CC BY-SA 4.0).

- CIVA (Comet Infrared and Visible Analyser)

- CONSERT (COmet Nucleus Sounding Experiment by Radio wave Trasmission)

- COSAC (COmetary SAmpling and Composition Experiment)

- MUPUS (MUlti-PUrpose Sensors for surface and sub-surface science)

- Ptolemy (geochemistry of light elements)

- ROLIS (ROsetta Lander Imaging System)

- ROMAP (ROsetta lander Magnetometer And Plasma monitor)

- SD2 (Sampling, Drilling and Distribution)

- SESAME (Surface Electric Sounding and Acoustic Monitoring Experiment)

Between 2005 and 2010, Rosetta performed several gravity assists around Earth and Mars, and flew passed two asteroids: on September 5, 2008, Rosetta flew by asteroid (2867) Steins (Glassmeier et al. 2007) and on July 10, 2010 around (21) Lutetia (Thomas et al. 2012). The next stop for Rosetta was the main target of the mission, comet 67P/Churyumov-Gerasimenko. After being in hibernation for three years, the spacecraft woke up in January 2014 and performed rendezvous manoeuvres at 4.5-4.0 AU distances in preparation for the start of the near-nucleus phase, which began in August 2014 (Glassmeier et al. 2007). The first Rosetta results come from this time in July-August 2014, some of which are summarised in Section 1.4 and 1.6.

On November 12, 2014, Rosetta delivered Philae to the comet surface. However, this part of the mission was not entirely successful. Due to a failure of the anchor harpoons, 


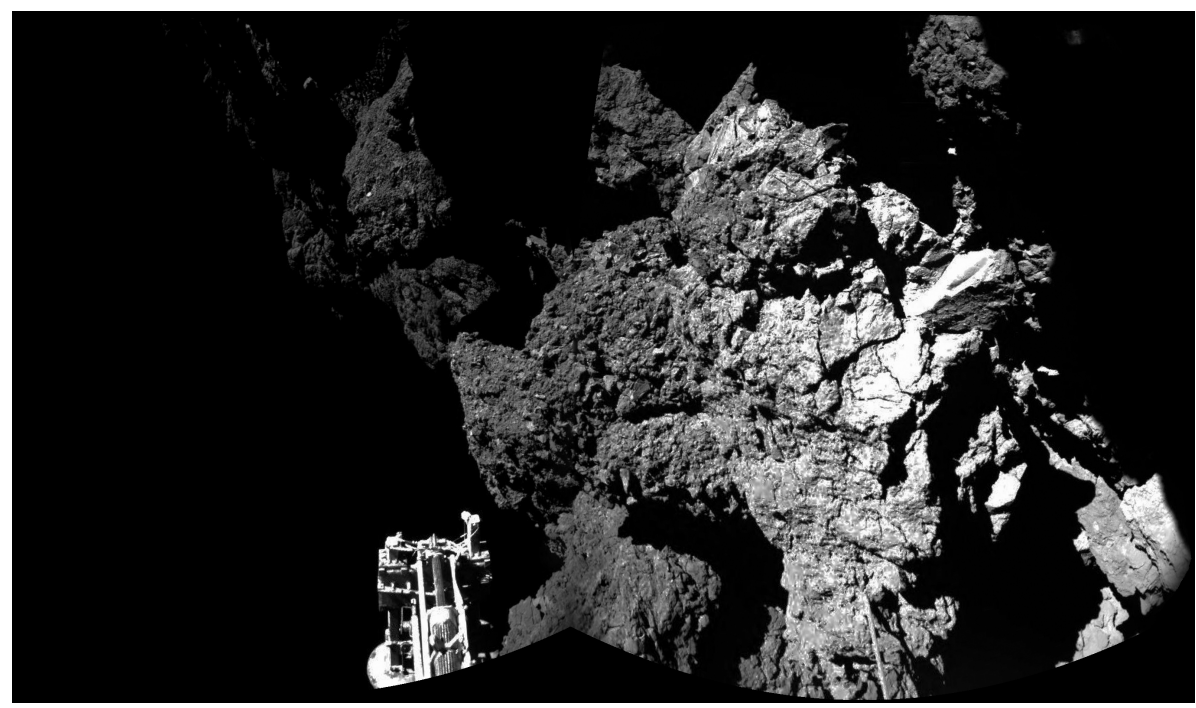

Figure 1.5: Image from the CIVA instrument on Philae of the comet surface (Credit: ESA/Rosetta/Philae/CIVA).

which did not fire, and the cold gas system, which could not push the lander onto the surface, Philae did not land as intended onto the surface and bounced several times (Biele et al. 2015). For a long time, Philae was thought to be lost until it was finally spotted in September 2016, lying on its sight on an uneven part of the comet surface (Schröder et al. 2017). Despite the suboptimal landing of Philae, it still managed to transmit many interesting close-up images of the nucleus as shown in Figure 1.5.

From November 2014 until September 2016, Rosetta escorted the comet along its perihelion passage, getting as close as 1.24 AU from the sun on August 13, 2015. The nominal end of the mission was supposed to be December 2015 (Glassmeier et al. 2007) but the performance of Rosetta allowed the mission to continue into 2016. The mission ended on September 30, 2016 with a controlled impact onto the nucleus.

Over the two year mission, Rosetta examined comet 67P/Churyumov-Gerasimenko in unprecedented detail and over an extended period of time. Never before had a comet been observed in such an intensive manner, and during the mission, some of its mysteries were revealed.

\subsection{P/Churyumov-Gerasimenko}

Comet 67P/Churyumov-Gerasimenko was first discovered in 1969 by Klim Ivanovich Churyumov and Svetlana Ivanova Gerasimenko (Lamy et al. 2007). Whilst working at the National Taras Shevchenko University of Kiev, Churyumov and Gerasimenko travelled to the Astorphysical Institute in Almaty, Kazakhstan to make observations of several short period and new comets. On Spetember 11, 1969, Gerasimenko attempted to observe comet $32 \mathrm{P} /$ Comas Solà but the photographic plate from their observation seemed to be defective. Nevertheless, an object in the frame appeared to be 32P/Comas Solà, so Gerasimenko kept the plate for analysis back in Kiev.

When they returned in October and studied their observations, Churyumov and Gerasi- 


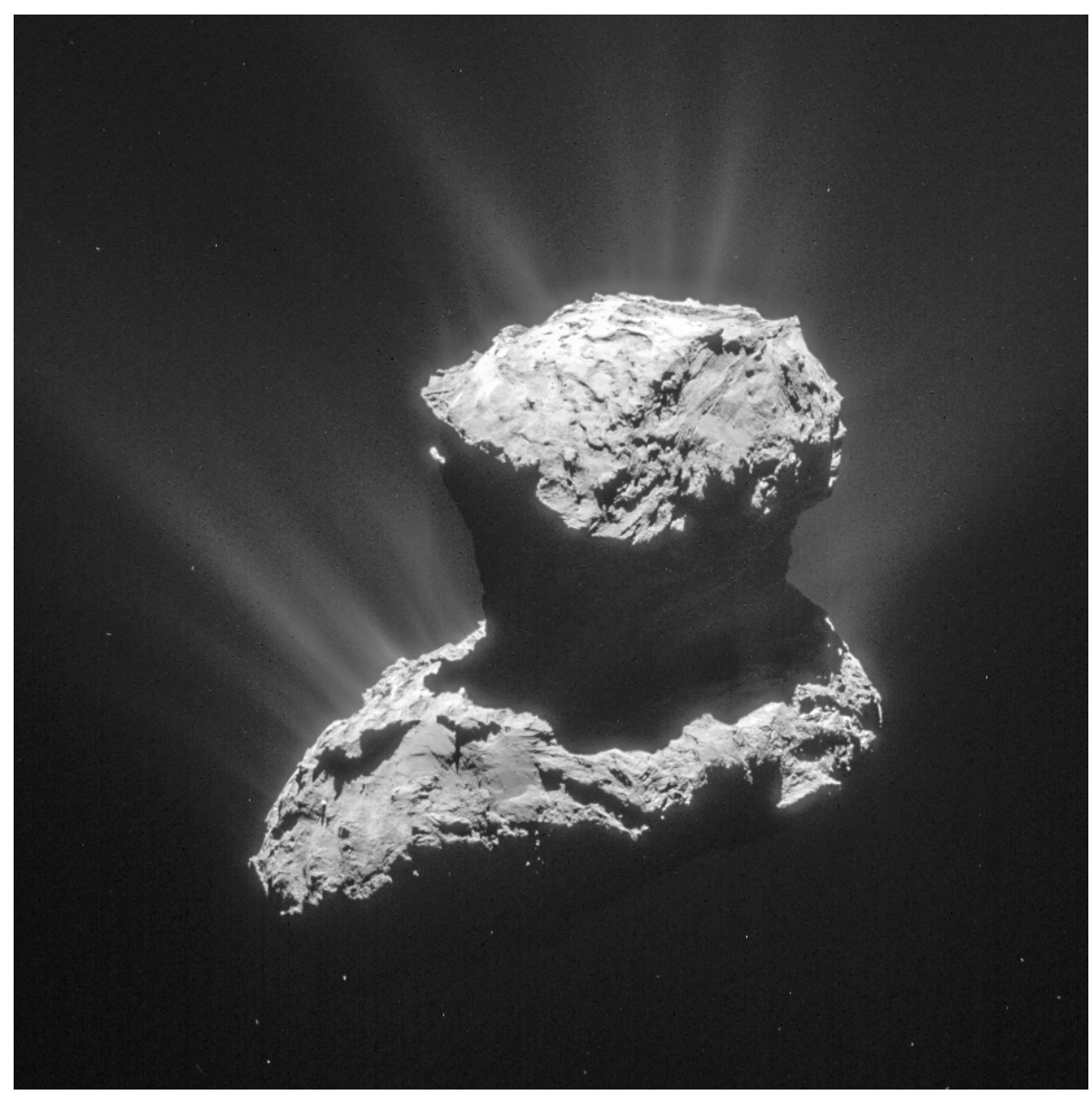

Figure 1.6: NavCam image of comet 67P/Churyumov-Gerasimenko from March 2, 2015 whilst Rosetta was at a distance of $90 \mathrm{~km}$ from the comet centre. The image has been enhanced to show the activity of the comet (Photo credit: ESA/Rosetta/NAVCAM, CC BY-SA IGO 3.0).

menko found that there were actually two comets in the plate from September 11: $32 \mathrm{P} /$ Comas Solà and another unidentified comet. They had inadvertently made four further observations of this new comet between September 9-21 and this new discovery was reported to the International Astronomical Union. The first sighting of this comet was then verified by John Bortle after studying an image from Charles Scovil taken on September 14, 1969, and the new comet became 67P/Churyumov-Gerasimenko.

Due to a favourable orbit, comet 67P had been seen as a potentially accessible object for a long time (Yeomans and Chodas 1989) but still relatively little was known about it at the time.

The orbit of comet 67P/Churyumov-Gerasimenko was found to have quite a chaotic history as a result of repeated encounters with Jupiter, and the last significant planetary encounter changed the orbit quite dramatically (Krolikowska 2003, Maquet 2015). The perihelion distance decreased from 2.74 to 1.28 , the eccentricity increased from 0.36 to 0.63 , and the orbital period decreased from 9 to 6.6 years. The orbit is reasonably well defined to 200 years into the future and 200 years into the past, but beyond that, encounters with the giant planets make it difficult to reconstruct the exact orbit. 


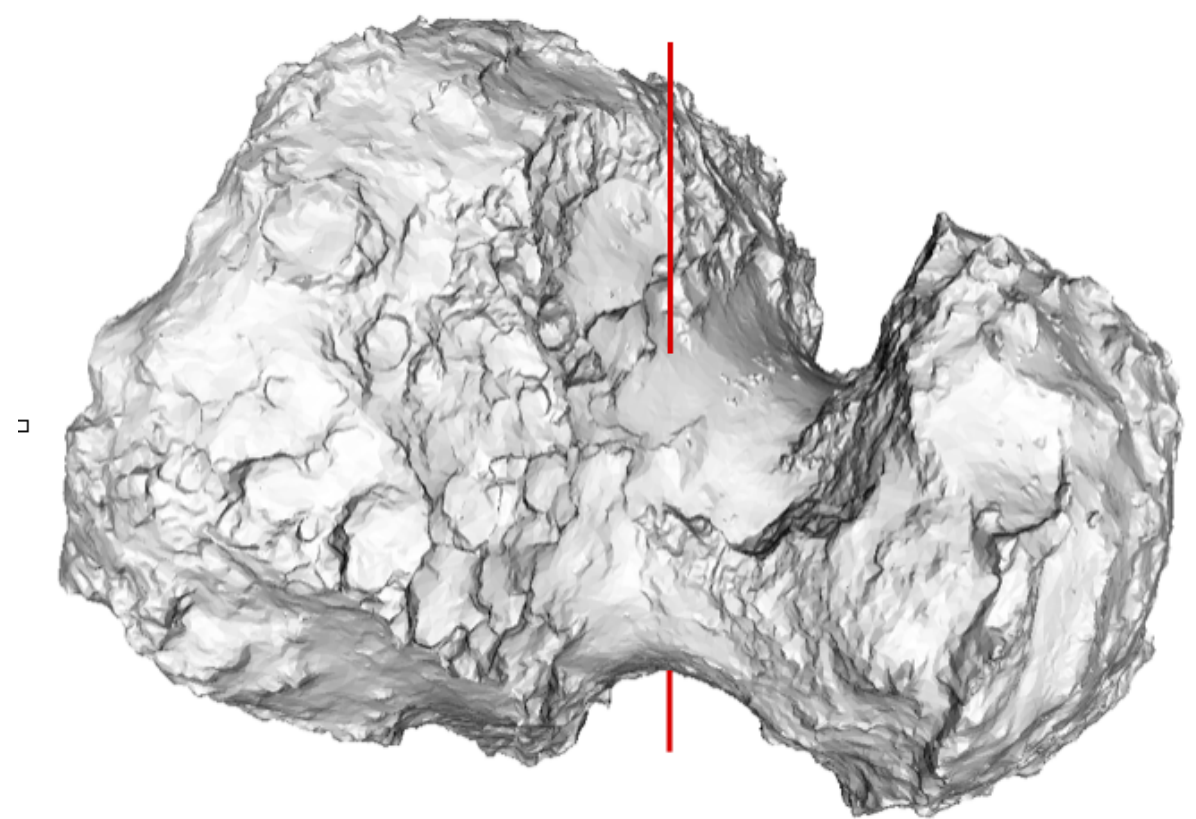

Figure 1.7: The shape model of comet 67P/Churyumov-Gerasimenko as found by (Preusker et al. 2017). The red line denotes the rotation axis which is tilted at $52^{\circ}$ and defines the latitude-longitude co-ordinate system on the surface.

Comet 67P/Churyumov-Gerasimenko attracted much more interest in 2003 when it was announced as the target comet for the Rosetta mission. Between the initial observations in 1969 and the announcement in 2003, the comet was only observed in three campaigns in 34 years but observation time was then found in 2003, 2004 and 2005 (Lamy et al. 2007). From initial lightcurve data taken by the Hubble Space Telescope in 2003, the shape of 67P/Churyumov-Gerasimenko was determined and found to be non-spherical (Lamy et al. 2006), the first indication of the unusual shape of comet 67P/ChuryumovGerasimenko. As a result of the mission, much more is known about the comet than any other small body in our solar system.

From the OSIRIS and navigational camera images, the detailed shape of comet 67P/Churyumov-Gerasimenko can be seen (Figure 1.6). By analysing over 1500 images, Preusker et al. (2017) used stereo-photogrammetric to derive a high resolution 3D model of the comet. Figure 1.7 shows the result of this work, although only a decimated version with 125,000 triangular is shown, rather than the full $1 \mathrm{~m}$ scale model with 44 million facets.

One of the most striking aspects of $67 \mathrm{P}$ is that it is a bilobed comet with a small head lobe and large body lobe which is in stark contrast to the more spherical comets previously observed in Figure 1.3. The origins of this shape are still unknown. Some argue that the bilobed comet is primordial as a result of low velocity accretion during the early formation of the solar system (Massironi et al. 2015, Jutzi and Asphaug 2015), but others say that based on its size, 67P would have experienced catastrophic collisions since then and that the shape need not be pristine (Morbidelli and Rickman 2015). The bilobed shape may then come from collisional disruptions later in the history of the solar system (Rickman et al. 2015, Jutzi and Benz 2017). This is still a matter of debate. 


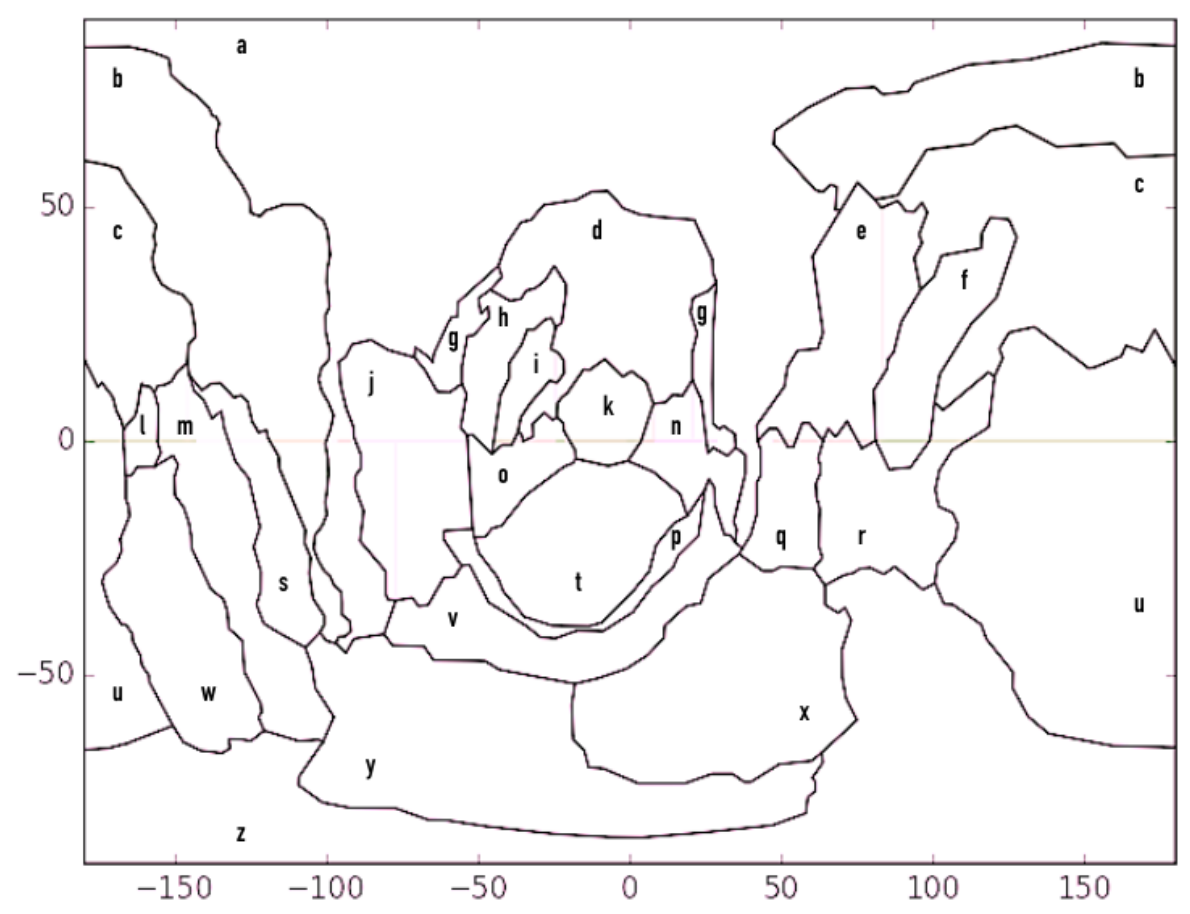

Figure 1.8: The regions on the surface of comet 67P/Churyumov-Gerasimenko as defined by (El-Maarry et al. 2015). These are: (a) Hapi, (b) Seth, (c) Ash, (d) Ma'at, (e) Babi, (f) Aten, (g) Hathor, (h) Serqet, (i) Nut, (j) Anuket, (k) Hatmehit, (l) Apis, (m) Atum, (n) Bastet, (o) Maftet, (p) Neith, (q) Aker, (r) Khepry, (s) Anubis, (t) Wosret, (u) Imhotep, (v) Sobek, (w) Khonsu, (x) Anhur, (y) Geb, (z) Bes.

OSIRIS also revealed the geological variety of the nucleus. As documented in ElMaarry et al. (2015) and El-Maarry et al. (2016), the surface of comet 67P/ChuryumovGerasimenko can be divided into 26 distinct morphological regions based on terrain and structure (Figure 1.8). For example, the Imhotep region is one of the most geologically diverse, with a smooth bouldered surface as well as rougher terrain, evidence of terracing, and filled and unfilled circular features. In contrast, Hathor on the head lobe is cliff-like, and has aligned lineaments and fractures. There are dust covered terrains (Ma'at and Ash regions) and regions of brittle consolidated material (Seth) (Thomas et al. 2015). Numerous features are apparent including depressions, wind tails and dune-like features, suggesting that a variety of processes occur on the comet surface.

The rotation axis of 67P is marked on Figure 1.7 with a red line. The poles are located in the neck region between the head and body lobes. From this axis, lines of longitude and latitude are defined as for Earth, allowing the map in Figure 1.8 to be made. However, due to the complex shape of $67 \mathrm{P}$, the lines of longitude and latitude are quite irregular. The equator runs around the body lobe, through the neck region and around the head lobe. The rotation axis is tilted at an angle of approximately $52^{\circ}$ of its orbital plane, and with such a severe tilt, there are pronounced seasonal effects between the north and south poles which will be discussed in Chapters 3 and 4.

The ROSINA spectrometer has also provided a wealth of information regarding the composition of the coma. ROSINA has the capabilities to measure the abundance of a 
host of oxygen, nitrogen and sulphur bearing molecules (Le Roy et al. 2015), and one of the key findings points towards a dichotomy in the $\mathrm{CO}$ and $\mathrm{CO}_{2}$ activity, with significant enhancements in these molecules from the southern hemisphere rather than the northern hemisphere. The ROSINA data also enabled the time variability of the abundance for a variety of species to be tracked (Hansen et al. 2016, Hässig et al. 2015), and the $\mathrm{D} / \mathrm{H}$ (deuterium/hydrogen) ratio to be determined, being 3 times higher than the terrestrial value and higher than that found for other Jupiter Family comets (Altwegg et al. 2015).

In addition, the VIRTIS instrument was able to identify the presence of non-volatile organic compounds on the surface of the comet from the observed low reflectance, spectral slopes, and the absorption feature between 2.9-3.6 $\mu \mathrm{m}$ (Capaccioni et al. 2015); while De Sanctis et al. (2015) were able to map the VIRTIS temperatures on the surface and hence derive a diurnal cycle for water to explain the observed outgassing. Another instrument, RSI, was used to determine the bulk mass and density of the comet $(9.982 \pm 0.003) \times 10^{12} \mathrm{~kg}$ and $(533 \pm 6) \mathrm{kg} \mathrm{m}^{-3}$ - from velocity perturbations at flyby distances and using the comet volume measured by Preusker et al. (2015). RSI also determined the global gravity field (Pätzold et al. 2016). In addition, this work implied that the interior must be fairly homogeneous, as did data from CONSERT (Kofman et al. 2015), which used the radar signal from Philae to find that the interior should be relatively homogeneous on $\sim 10 \mathrm{~m}$ scales.

The dust component of 67P/Churyumov-Gerasimenko has also been characterised by various instruments onboard Rosetta and Philae. There is evidence for a dusty crust (Schulz et al. 2015) from the GIADA instrument, which also measured the mass distribution of dust grains (in the range $10^{-10}-10^{-2} \mathrm{~kg}$ ) and the dust-gas ratio, finding a value of $4 \pm 2$ (Rotundi et al. 2015). COSIMA was able to collect and analyse the dust particles and determined that there are whole typological classes of dust, with many of them in clusters, and similar to micrometeorites and interplanetary dust particles (Langevin et al. 2016).

The results briefly mentioned here are only a fraction of the findings that the Rosetta mission has revealed about comet 67P/Churyumov-Gerasimenko. Much more research has been done, and will be done, with the data from these instruments as well as others, including MIRO, the Microwave Instrument for the Rosetta Orbiter.

\subsection{The Microwave Instrument for the Rosetta orbiter}

The Microwave Instrument for the Rosetta Orbiter (MIRO), was one of the eleven instruments onboard the Rosetta spacecraft. This section provides a brief overview of the instrument, but the full technical details can be found in Gulkis et al. (2007).

MIRO consisted of a primary $30 \mathrm{~cm}$ reflector telescope and two heterodyne receivers operating at centre band frequencies of $190 \mathrm{GHz}(1.6 \mathrm{~mm})$ and $562 \mathrm{GHz}(0.5 \mathrm{~mm})$. These are respectively referred to as the millimetre and submillimetre receivers hereafter. Both receivers had broadband continuum channels for the measurement of sub surface brightness temperatures. The brightness temperature is defined as the required temperature of a blackbody which fills the MIRO beam to produce the observed power. MIRO measured an antenna temperature, $T_{A}$, first, which was calculated from the received power, $P$, the Boltzmann constant, $k$, and the bandwidth, $d v$ : 
Table 1.1: Molecular transitions observed by the MIRO submillimetre spectrometer, reproduced from Gulkis et al. (2007)

\begin{tabular}{|c|c|c|}
\hline Molecule & Frequency $(\mathrm{GHz})$ & Transition \\
\hline $\begin{array}{c}\text { Ammonia } \\
\mathrm{NH}_{3}\end{array}$ & 572.498 & $\mathrm{~J}(1-0)$ \\
\hline $\begin{array}{c}\text { Carbon Monoxide } \\
\mathrm{CO}\end{array}$ & 576.268 & $\mathrm{~J}(5-4)$ \\
\hline Methanol & & \\
$\mathrm{CH}_{3} \mathrm{OH}$ & 553.146 & $8(1)-7(0) \mathrm{E}$ \\
$\mathrm{CH}_{3} \mathrm{OH}$ & 568.566 & $3(-2)-2(-1) \mathrm{E}$ \\
$\mathrm{CH}_{3} \mathrm{OH}$ & 579.151 & $12(-1)-11(-1) \mathrm{E}$ \\
\hline $\mathrm{Water}^{\mathrm{H}}{ }_{2}^{16} \mathrm{O}$ & 556.936 & $1(1,0)-1(0,1)$ \\
$\mathrm{H}_{2}{ }^{17} \mathrm{O}$ & 552.021 & $1(1,0)-1(0,1)$ \\
$\mathrm{H}_{2}{ }^{18} \mathrm{O}$ & 547.676 & $1(1,0)-1(0,1)$ \\
\hline
\end{tabular}

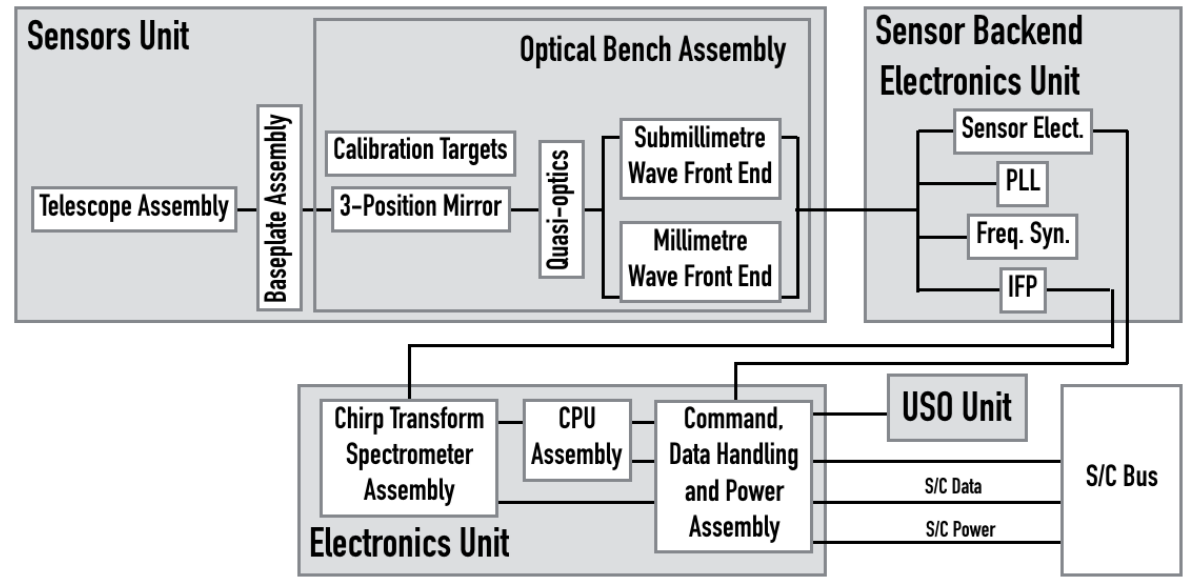

Figure 1.9: Schematic representation of the MIRO units. There are four units: Sensors Unit, Sensor Backend Electronics Unit, Electronics Unit and an Ultra-Stable Oscillator (USO) Unit. The Sensors Unit contained the telescope, baseplate and the optical bench (the calibration targets, the 3-position mirror, the quasi-optics, and the two heterodyne receivers). The Sensor Backend Electronics Unit held the Intermediate Frequency Processor (IFP), phase lock loop, sensor electronics and frequency synthesisers. The Electronics Unit contained the Chirp Transform Spectrometer, the instrument computer, power circuits and spacecraft interface circuits. The USO Unit was a self contained unit which held the oscillators used in the downconversion of the received signal. Image inspired by (Gulkis et al. 2007). 


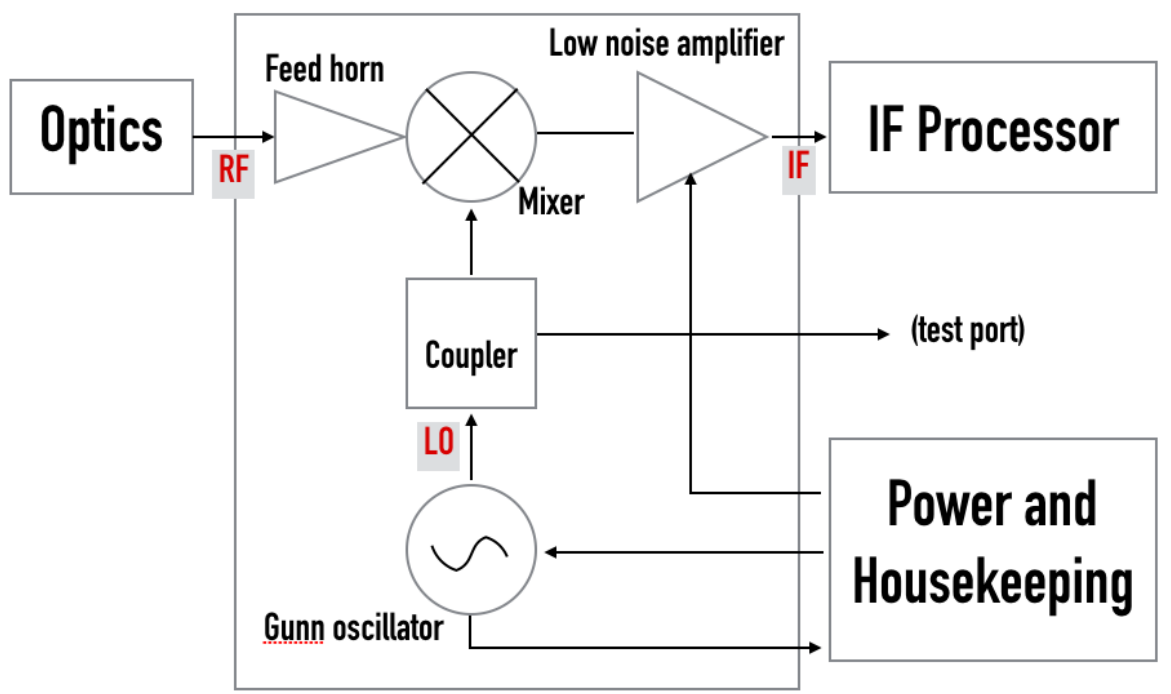

Figure 1.10: Technical diagram of the heterodyne receivers of MIRO. The front ends of the millimetre and the submillimetre receivers both have the same design. The power is detected at RF radio frequencies and sent from the optical bench to a subharmonically pumped mixer via a feed horn. At the mixer, the RF signal is multiplied by a signal (LO) from a low frequency oscillator. The LO frequency is produced by a Gunn oscillator. The mixer produces an IF Intermediate Frequency which is passed through a low noise amplifier to an IF processor. All of this is controlled by the power unit. Image inspired by (Gulkis et al. 2007).

$$
T_{A}=\frac{P}{k d v}
$$

The antenna temperature can then be converted to a brightness temperature, $T_{B}$, using:

$$
T_{B}(v)=\frac{1}{k} \frac{h v}{\ln \left(\frac{h v}{k T_{A}(v)}+1\right)}
$$

where $v$ is the frequency and $h$ is the Planck constant.

In addition, a Chirp Transform Spectrometer (hereafter, CTS, Hartogh and Hartmann (1990)) was attached to the submillimetre receiver for the detection of molecular spectral lines. The CTS was capable of detecting absorption and emission lines from some of the most abundant species previously observed in comets, including: carbon monoxide (CO); methanol $\left(\mathrm{CH}_{3} \mathrm{OH}\right)$; ammonia $\left(\mathrm{NH}_{3}\right)$; and three isotopologues of water $\left(\mathrm{H}_{2}{ }^{16} \mathrm{O}, \mathrm{H}_{2}{ }^{17} \mathrm{O}\right.$, $\mathrm{H}_{2}{ }^{18} \mathrm{O}$ ). The transitions for these molecular lines are listed in Table 1.1.

MIRO consisted of four units (Figure 1.9): Sensors Unit, Sensor Backend Electronics Unit, Electronics Unit and an Ultra-Stable Oscillator Unit (Gulkis et al. 2007). The Sensors Unit contained the telescope, baseplate and the optical bench. The two heterodyne receivers were part of the optical bench, along with the hot and cold calibration targets. The Sensor Backend Electronics Unit held the Intermediate Frequency Processor (IFP), phase lock loop, housekeeping circuits and frequency synthesisers. The CTS was part 
of the Electronics Unit, as well as the instrument computer, power circuits and spacecraft interface circuits. Finally, the Ultra-Stable Oscillator Unit was a self contained unit which held the oscillators used in the downconversion of the received signal. Figure 1.9 shows how the signal flows from the telescope, via the heterodyne receivers, to the IFP and the spectrometer.

The primary $30 \mathrm{~cm}$ offset parabolic reflector telescope had a focal length of $37.5 \mathrm{~cm}$ and formed the external part of the Sensors Unit, with the optical bench mounted inside the spacecraft. The telescope was located on the top of Rosetta and aligned with the payload line of sight. The angular resolution for the millimetre and submillimetre receivers were 23.8 arcmin $(6.9 \mathrm{mrad})$ and $7.5 \operatorname{arcmin}(2.2 \mathrm{mrad})$, respectively. At an altitude of $30 \mathrm{~km}$ from the nucleus, the beams would have sizes of $200 \mathrm{~m}$ in the millimetre channel and $70 \mathrm{~m}$ in the submillimetre channel. Light from the primary telescope was reflected inside to the optical bench. At the optical bench, the incoming light was split into millimetre and submillimetre components and directed to two heterodyne receivers.

Heterodyne receivers work by mixing (multiplying) the received frequency, called RF (radio frequency), with the frequency of a local oscillator (LO). The multiplication of the RF and LO frequencies provides sum and difference frequencies called intermediate frequencies (IF). Usually, the IF is used for further processing and it can be chosen in a way that it appears in a range where electronic processing is feasible (Janssen 1994). In the case of MIRO, the RF needed to be downconverted to lower frequencies. There are a number of reasons why it can be beneficial to shift the frequency range lower. Circuitry performance is relatively poor at high frequencies and transistors provide little amplification.

Both the millimetre and submillimetre heterodyne receivers worked in the same way and are shown schematically in Figure 1.10. The RF signal arrived from the optics unit and went into a subharmonically pumped mixer via a feed horn. At the mixer, the RF signal was multiplied by the LO signal to downconvert the signal to the IF. The LO was provided by a Gunn oscillator which operated in a frequency switching mode. In this mode, the Gunn oscillator frequency switched by $\pm 5 \mathrm{MHz}$ every 5 seconds, creating shifts in the spectral lines. The shifts could be used to remove gain and baseline variations which occur in the receiver and thus improve the sensitivity of the instrument. The IF signal was then amplified and finally sent to the IFP for detection. The millimetre and submillimetre RFs were in the range 186.7-189.7 GHz and 547.6-579.2 Hz, respectively, whilst the LOs were at $\sim 95 \mathrm{GHz}$ and $\sim 282 \mathrm{GHz}$ (Gulkis et al. 2007). The IFs then had ranges of $1-1.5 \mathrm{GHz}$ and $5.5-16.5 \mathrm{GHz}$, respectively.

The IFP had two continuum channels, one for each receiver, and prepared the spectral bands for the CTS (Hartogh and Hartmann 1990). The CTS was a 4096 channel spectrometer with a $180 \mathrm{MHz}$ bandwidth and a spectral resolution of $44 \mathrm{kHz}\left(v / \Delta v \sim 10^{7}\right)$. The spectrometer enabled the detection of the eight molecular transitions listed in Table 1.1. The lines are separated by a filterbank at the input of the CTS. Table 1.2 details how the RF was downconverted to the bandwidth of the CTS through a series of LO. The downconversion to IF1 is performed in the submillimetre receiver. The remaining IFs are produced in the IFP, which uses nine mixers and three LO sources to complete the downconversion. The bandwidth of the RF reduces from $31.6 \mathrm{GHz}$ to $180 \mathrm{MHz}$ in the CTS. The MIRO CTS spectrometer was uniquely capable of making high resolution observations of the intrinsic narrow Doppler broadened spectral lines which result from 
Table 1.2: Downconversion of the radio frequency RF (by multiplication with local oscillator frequencies LO) for each molecular transition given in Table 1.1. The RF is downconverted to a series of intermediate frequencies IF and finally to the bandwidth of the CTS (Hartogh and Hartmann 1990). All frequencies given in units of MHz. Reproduced from Gulkis et al. (2007)

\begin{tabular}{|c|c|c|c|c|c|c|c|c|}
\hline & $\mathrm{H}_{2}{ }^{16} \mathrm{O}$ & $\mathrm{H}_{2}{ }^{16} \mathrm{O}$ & $\mathrm{CH}_{3} \mathrm{OH}$ & $\mathrm{H}_{2}{ }^{18} \mathrm{O}$ & $\mathrm{CO}$ & $\mathrm{CH}_{3} \mathrm{OH}$ & $\mathrm{NH}_{3}$ & $\mathrm{CH}_{3} \mathrm{OH}$ \\
\hline RF & 556936 & 552022 & 579151 & 547676 & 576268 & 568566 & 572498 & 553146 \\
IF 1 (LO 1) & 5877 & 10791 & 16338 & 15137 & 13455 & 5753 & 9685 & 9667 \\
IF 2 (2×LO2) & - & 6427 & - & - & 9091 & 1389 & 14049 & 14031 \\
IF 3 (LO4) & - & - & - & - & 1363 & - & 6321 & 6303 \\
IF 4 (LO4) & - & - & - & - & - & - & 1407 & 1425 \\
IF 5 (LO3) & - & 1301 & - & - & - & - & - & - \\
IF 6 (LO2) & 1270 & - & 9191 & 7990 & - & - & - & - \\
IF 7 (LO3) & - & - & - & 5808 & - & - & - & - \\
IF 8 (2×LO2) & - & - & 2044 & 1339 & - & - & - & - \\
IF 9 (LO4) & - & - & 6408 & - & - & - & - & - \\
IF 10 & - & - & 1320 & - & - & - & - & - \\
output to CTS & 1270 & 1301 & 1320 & 1339 & 1363 & 1389 & 1407 & 1425 \\
\hline
\end{tabular}

the low gas temperatures and low pressures around comets.

The large dataset provided by MIRO allows measurements of molecular column densities and production rates to be made from the observed spectral lines, as well as the kinetic properties within the inner coma. The MIRO brightness temperatures derived from the continuum channel also enable the thermal properties of the subsurface to be inferred. The temporal and spatial capabilities of the instrument allow these measurements to be taken across the whole surface of the nucleus and throughout the entire two year mission.

\subsection{MIRO aims, results and spectra}

Before the beginning of the Rosetta mission, several measurement objectives were outlined for the MIRO instrument in Gulkis et al. (2007). One objective was to measure the abundances of some of the major volatile species detectable by MIRO. The sublimation of $\mathrm{H}_{2} \mathrm{O}$ and $\mathrm{CO}$ are thought to be two of the main drivers of activity in comets and during the lifetime of Rosetta, the change in abundance of these two key volatiles can be measured as $67 \mathrm{P} /$ Churyumov-Gerasimenko approaches and recedes from the sun after perihelion.

Another important objective related to the nucleus itself, as the MIRO data can help to improve our understanding of the processes which control cometary activity in the subsurface layer, and hence characterise the surface over the first few centimetres. Knowledge of the thermal gradient in the upper layers of the nucleus can help to constrain the physical nature of the comet and determine how much material could still be pristine from the early solar system.

Furthermore, MIRO offered a unique opportunity to study the evolution and development of the inner coma. This region is impossible to probe from ground-based telescopes or in situ instruments, but as a remote sensing unit, the MIRO measurements can be used 
to estimate the kinetic temperature, expansion velocity and molecular density along its line of sight. This will enable the structure and dynamics of the inner coma to be studied in great detail.

The final main objective was to take advantage of the fly-bys of asteroids Lutetia and Steins to probe for low levels of gas in these objects. This was accomplished by Gulkis et al. (2010) and Gulkis et al. (2012).

Much of the early MIRO work regarding 67P went into characterising the surface by looking at the subsurface temperatures. The submillimetre and millimetre emissions are estimated to come from depths of $1 \mathrm{~cm}$ and $4 \mathrm{~cm}$ respectively (Schloerb et al. 2015). Alternatively, Choukroun et al. (2015) propose that the depths of these brightness temperatures may originate from much deeper layers if ice is present in the surface, which could extend the electrical penetration depth, giving rise to potential depths of $15-20 \mathrm{~cm}$ in the sub-millimetre channel and $20-30 \mathrm{~cm}$ in the millimetre channel for icy regions. Schloerb et al. (2015) and Gulkis et al. (2015) were able to demonstrate the diurnal variation of the subsurface temperatures when $67 \mathrm{P} /$ Churyumov-Gerarsimenko was more than $3 \mathrm{AU}$ away from the sun. The variation appears to be a function of latitude, owing to the fact that the received solar flux strongly depends on the latitude, and there is a possible temperature lag, with the highest subsurface temperatures occurring in the early or mid afternoon, local time. Night time subsurface temperature measurements are given in Choukroun et al. (2015), showing the surface to be very cold, between 17-50 K across both instrument channels. The low sub-surface temperatures can only be measured precisely with a millimetre/sub-millimetre instrument like MIRO operating at this wavelength range.

Schloerb et al. (2015), Choukroun et al. (2015) and Gulkis et al. (2015) made estimates for the thermal inertia of the surface, the ability of the surface to resist changes in temperature. Materials with a high thermal inertia, such as rocks, change temperature slowly, whereas materials with a low thermal inertia, like sand, thermalise quickly. A thermal inertia in the range $10-30 \mathrm{JK}^{-1} \mathrm{~m}^{-2} \mathrm{~s}^{-0.5}$ for the surface is found by Schloerb et al. (2015), $10-50 \mathrm{JK}^{-1} \mathrm{~m}^{-2} \mathrm{~s}^{-0.5}$ from Gulkis et al. (2015), and from Choukroun et al. (2015), $10-40 \mathrm{JK}^{-1} \mathrm{~m}^{-2} \mathrm{~s}^{-0.5}$ in the sub-millimetre channel and $20-60 \mathrm{JK}^{-1} \mathrm{~m}^{-2} \mathrm{~s}^{-0.5}$ in the millimetre channel. The discrepancy between the two channels is found when Choukroun et al. (2015) try to fit their model to the millimetre and sub-millimetre measurements independently, thus obtaining slightly different thermal inertia ranges. They suggest that the presence of ice in the sub-surface, which has a wavelength dependent electrical penetration depth, could explain the difference.

As well as the nucleus, the MIRO data also offers an opportunity to probe the coma around the comet. Using parametrised coma profiles of temperature, velocity and density, Lee et al. (2015) measured the outgassing rate, the terminal gas expansion velocity and the terminal gas temperature across the nucleus from measurements made in August 2014. Interestingly, they found that the outgassing of the comet was not strongly correlated to the solar illumination or the surface temperature, implying that illumination is not the only driver of cometary activity. It is revealing that Lee et al. (2015) report an increase in the outgassing just after sunset on the comet, suggesting that the deposits of ice must be below the surface but within the penetration depth of the thermal wave. In addition, they find that the neck is the most active region at this time, similar to findings by other instruments. The spatial variation results in some areas which have 30 times more water production than less active areas. The temporal variation changes by only a factor of 


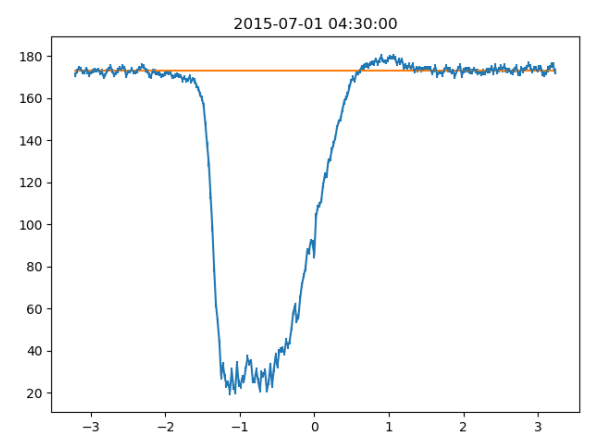

(a)

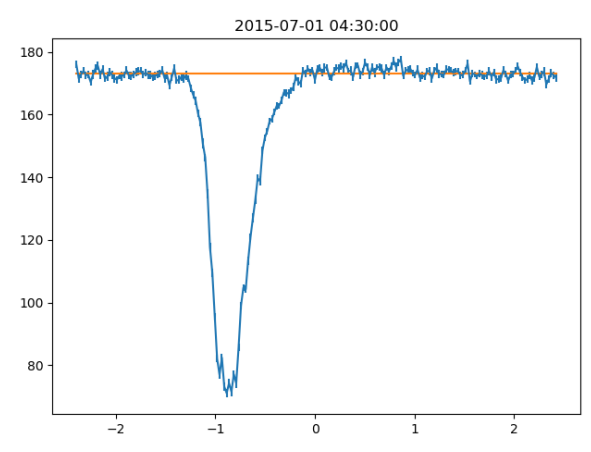

(c)

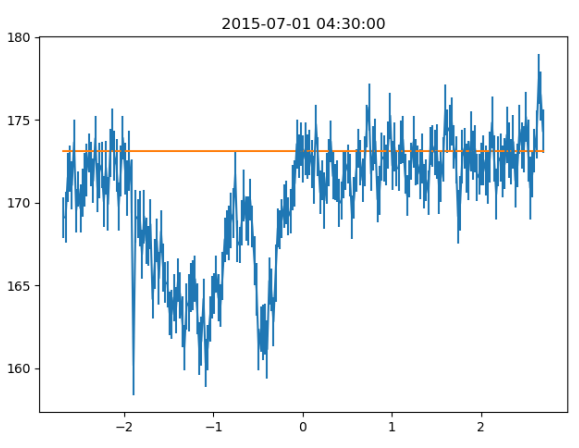

(b)

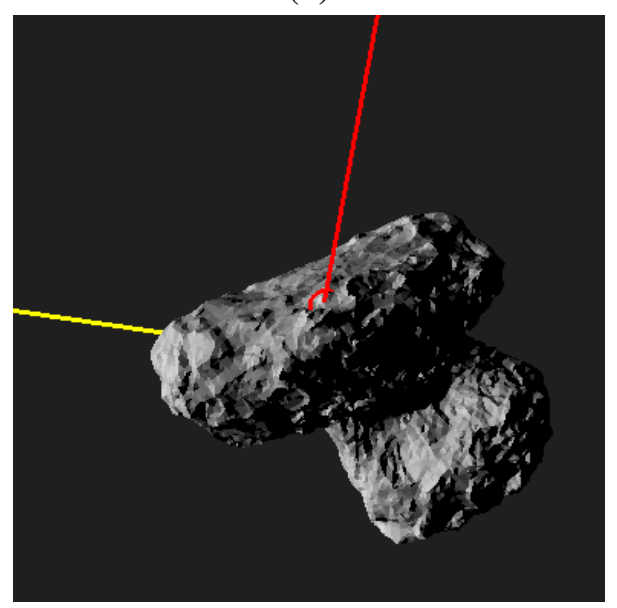

(d)

Figure 1.11: Spectral lines observed by MIRO of (a) $\mathrm{H}_{2}{ }^{16} \mathrm{O}$, (b) $\mathrm{H}_{2}{ }^{17} \mathrm{O}$, and (c) $\mathrm{H}_{2}{ }^{18} \mathrm{O}$, on July 1, 2015 at 04:45 with a 30 minute integration time. Doppler shift velocity is depicted on the $\mathrm{x}$ axis and antenna temperature on the $\mathrm{y}$ axis. The measured continuum temperature for these observations was $173 \mathrm{~K}$. The viewing geometry is shown in (d) with the red line and circle indicating the MIRO beam and the MIRO footprint, respectively. The yellow line is the vector towards the sun.

2 though, so the spatial distribution is much more prevalent than the temporal changes during this early study period.

Biver et al. (2015) also derived the column densities around the nucleus in the early part of the mission from the MIRO spectra. They are able to map the the spectra to the pointing of the spacecraft and thus create a map of temperature, expansion velocity and water column densities around comet 67P/Churyumov-Gerasimenko. The results are similar to Lee et al. (2015), in that the neck region is found to be the origins of higher column densities than other parts of the comet. There is also evidence for low levels of production on the night side, although this could also be a result of back flow towards the dark unilluminated side.

Figure 1.11 shows examples of the spectral lines observed by MIRO of three water isotopologues from observations at $04: 45$ on July 1, 2015 with an integration time of 30 minutes. $\mathrm{H}_{2}{ }^{18} \mathrm{O}$ and $\mathrm{H}_{2}{ }^{17} \mathrm{O}$ are minor species of water with terrestrial abundance ratios of $\frac{1}{498.7}$ and $\frac{1}{2632}$, respectively, relative to $\mathrm{H}_{2}{ }^{16} \mathrm{O}$ (Baertschi 1976, Jabeen and Kusakabe 


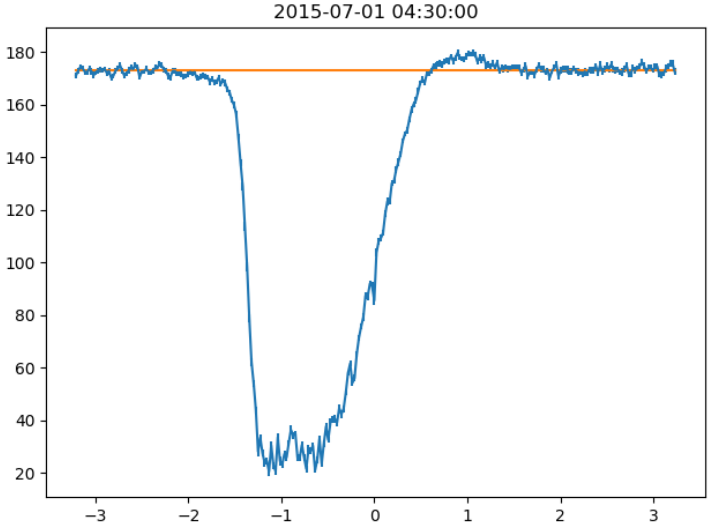

(a)

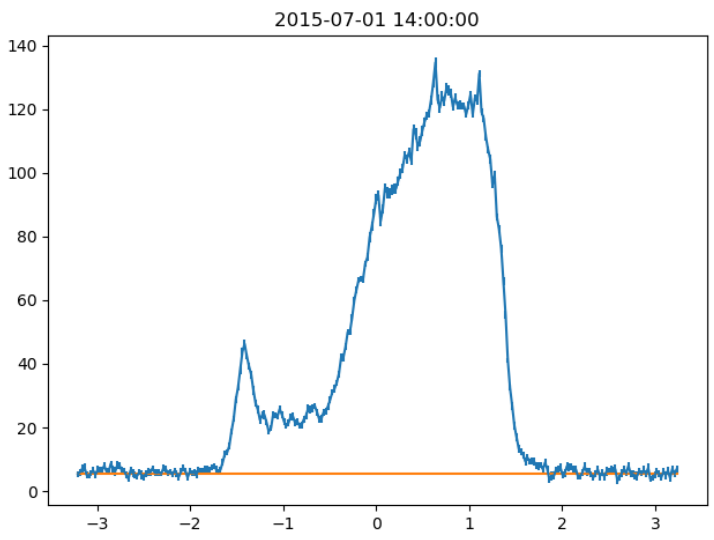

(c)

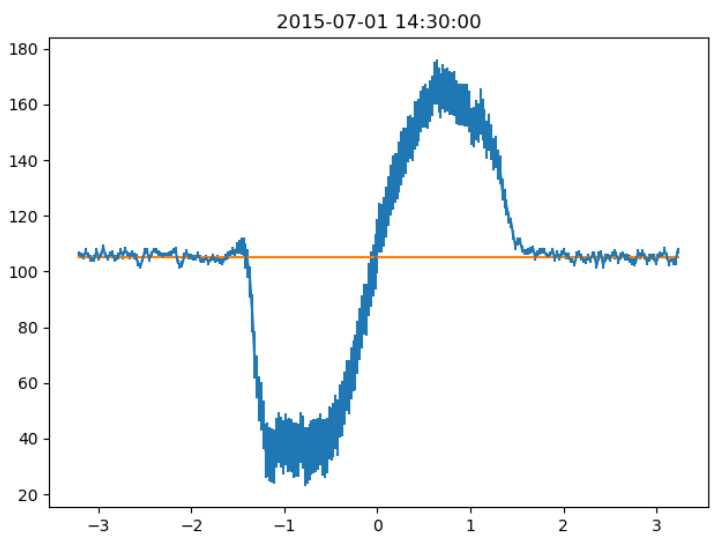

(e)

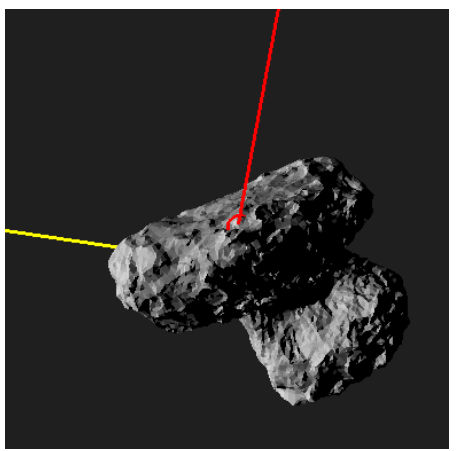

(b)

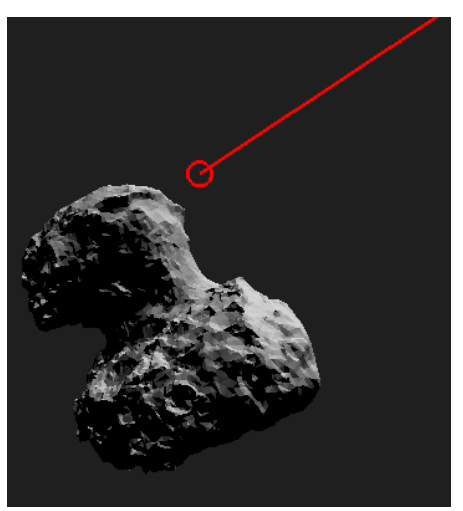

(d)

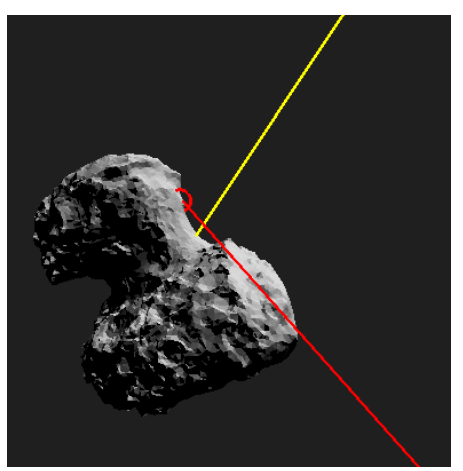

(f)

Figure 1.12: Spectral lines and viewing geometry of observations from MIRO on July 1, 2015 with 30 minute integration time. (a) $\mathrm{H}_{2}{ }^{16} \mathrm{O}$ in absorption against warm nucleus from 04:30-05:00, subsurface temperature of $173 \mathrm{~K}$ and viewing geometry shown in (b) which is the same as in Figure 1.11. (c) $\mathrm{H}_{2}{ }^{16} \mathrm{O}$ in emission against cold space from 14:0014:30, background temperature $\sim 5 \mathrm{~K}$ and viewing geometry shown in (d). (e) $\mathrm{H}_{2}{ }^{16} \mathrm{O}$ in absorption and emission MIRO observes nadir and limb simultaneously as the viewing geometry shows in (f). The viewing geometry shows a red line and circle indicating the MIRO beam and the MIRO footprint, respectively. The yellow line is the vector towards the sun. 
1997). They are shown here in absorption against the warm nucleus surface. All of these molecules consist of two hydrogen atoms and an oxygen atom but they differ in the number of neutrons within the oxygen atom, denoted by the superscript number. The $\mathrm{H}_{2}{ }^{16} \mathrm{O}$ line, the main species of water is shown in Figure 1.11a, and the wide deep shape of the spectral line shows that it is saturated and optically thick. The $\mathrm{H}_{2}{ }^{18} \mathrm{O}$ line shown in Figure 1.11c is optically thinner, thus narrower and having a lower contrast than the $\mathrm{H}_{2}{ }^{16} \mathrm{O}$ line. The spectrum in Figure $1.11 \mathrm{~b}$ shows the hyperfine structure in the $\mathrm{H}_{2}{ }^{17} \mathrm{O}$ line. It is five times less abundant than the $\mathrm{H}_{2}{ }^{18} \mathrm{O}$ and the line is weaker by this amount (requiring 25 times the observing time). Figure 1.11d shows the viewing geometry of this observation, which at this time was in a terminator orbit, observing close to the transition from day to night side.

The MIRO spectral lines can be observed in absorption and emission, as seen in Figure 1.12 for the optically thick $\mathrm{H}_{2}{ }^{16} \mathrm{O}$ line. In Figure 1.12a, MIRO makes a nadir observation and the line is seen in absorption against the warm nucleus with a temperature of $173 \mathrm{~K}$ on July 1, 2015 at 04:30. The viewing geometry is shown in Figure 1.12b and is for the same time as in Figure 1.11. Due to the high optical depth $(>10)$, the line core is saturated, and the velocity profiles makes the line shape asymmetric. There is also evidence of an emission wing at $\sim 1 \mathrm{~km} / \mathrm{s}$.

The $\mathrm{H}_{2}{ }^{16} \mathrm{O}$ line in emission from a limb observation is shown in Figure 1.12c with the viewing geometry in Figure 1.12d. The emission line is quite complex owing to the optical thickness and the velocity profile. The high opacity line creates the double peaked features, where an optically thin line would be approximately Gaussian in shape. The non-constant velocity profile then skews the emission line, making it asymmetric. The line core in this example occurs at $\sim-1 \mathrm{~km} / \mathrm{s}$.

Figure 1.12f shows MIRO observing partly on and partly off the nucleus, and as a result, the spectra in this time period exhibits both absorption and emission features (Figure $1.12 \mathrm{e})$ since it is a convolution of nadir and limb measurements.

\subsection{Thesis aims}

In this work, I explore aspects of the first three aims outlined in the previous section. In Chapter 3, the abundance of water is measured by tracking the change in the line area ratio of the $\mathrm{H}_{2}{ }^{16} \mathrm{O}$ and $\mathrm{H}_{2}{ }^{18} \mathrm{O}$ lines to find spatially resolved local water production rates. This is done from $\sim 3 \mathrm{AU}$ pre-perihelion to $\sim 2 \mathrm{AU}$ post-perihelion, allowing the change in production rate to be seen during the comets close approach to the sun. The meaning of water production rate curves and their derived slopes is investigated in Chapter 4. Chapter 5 describes how the thermal gradient can be found from the MIRO brightness temperature measurements, and hence derive estimates for the thermal inertia of the comet surface. In the chapter on Future Work, the derivation of temperature, velocity and density profiles in the inner coma is discussed, showing how the spectra can be inverted to determine these parameters. Finally, in Chapter 7, the results presented in the previous chapters are briefly summarised in their wider context.

The next chapter contains a brief introduction to a few scientific concepts which will occur in later chapters. 


\section{Theoretical concepts}

In this chapter, a few basic physical and theoretical concepts will be introduced which will be mentioned in future chapters.

\subsection{Haser model}

The Haser model is the simplest and most widely used model to describe the outflow of gas from a comet nucleus. It assumes a spherical comet has a global production rate for parent molecular species and that there is an isotropic outflow of material with finite lifetimes for the gas. The density distribution of gas produced from the nucleus is given by the equation:

$$
N(r)=\frac{Q}{4 \pi r^{2} v} \exp (-r / R)
$$

where $N(r)$ is the density at a distance, $r, Q$ is the production rate of the gas in [molecules $\mathrm{s}^{-1}$ ], $v$ is the expansion velocity of the gas into the coma, and $R$ is the scale length of the gas, given by $R=v t$ where $t$ is the lifetime of the molecule (De Pater and Lissauer 2015). Here, the velocity profile is assumed to be constant and move radially outwards, although the velocity can also depend on the distance from the comet. For molecules with a long lifetime and thus long scale length, the density yields a $1 / r^{2}$ dependence when following the assumptions given above.

The density distribution of daughter species can also be calculated with the Haser model if the lifetimes and production pathways of these daughter species are known as well.

The Haser model is thought to be a reasonable first approximation when deriving the production rate from comets (Snodgrass et al. 2017), and it has been reasonably successful in the past for deriving the density around a nucleus (Cochran 1985). However, the assumptions stipulated above, such as sphericity and radial outflow, can lead to problematic attempts to fit observed spectra with the Haser model formalism and other methods can be more suitable (Bockelée-Morvan and Gérard 1984).

\subsection{A two level atom}

An atom with two energy levels is represented in Figure 2.1. It has a lower level $l$ with an energy $E_{l}$ and an upper level $u$ with an energy $E_{u}$. Photons can be absorbed and emitted via transitions between the two energy levels. There are electronic transitions, vibrational 


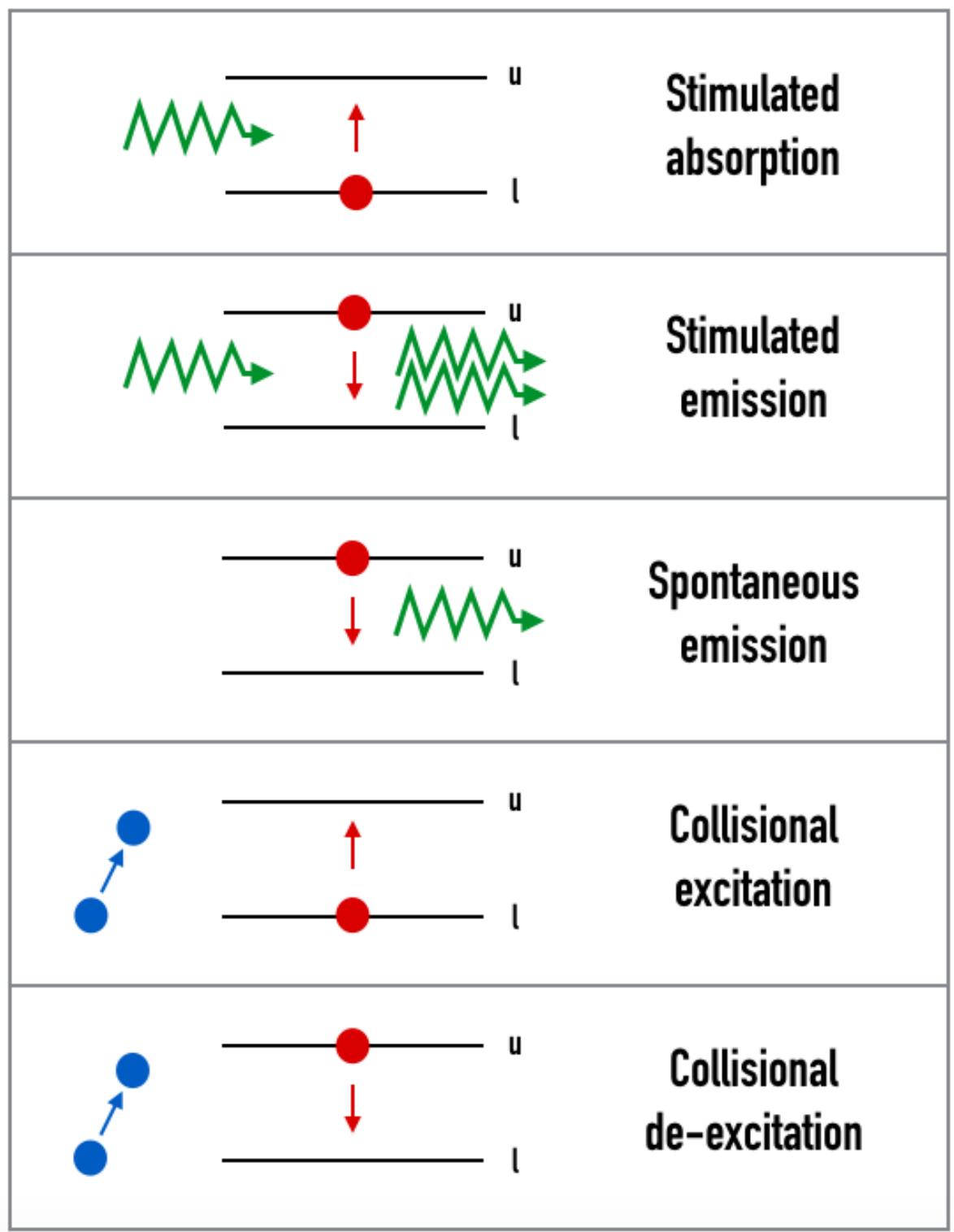

Figure 2.1: Schematic representation of an energy level system in an atom. Stimulated absorption - a background radiation field inducing a transition from the lower, $l$, level to the upper, $u$, level through the absorption of a photon with energy $h v_{0}=E_{u}-E_{l}$. Stimulated emission - a background radiation field inducing a transition from the upper level to the lower level resulting in the emission of a photon with energy $h v_{0}=E_{u}-E_{l}$. Spontaneous emission - a spontaneous transition takes place between the upper and lower energy levels resulting in the emission of a photon. Collisional excitation - collisions between atoms result in a transition from the lower state to the upper state. Collisional de-excitation - collisions between atoms result in a transition from the upper state to the lower state. 
transitions and rotational transitions. Rotational transitions are particularly important for the microwave regime.

The first case demonstrates stimulated absorption, whereby the energy from an incoming photon is absorbed by the atom and there is a transition from the lower level to the upper level. This is controlled by the Einstein coefficient $B_{l u}\left[\mathrm{~J} \mathrm{~s}^{-1} \mathrm{~m}^{2} \mathrm{sr} \mathrm{molec}^{-1}\right]$ which is the probability per second per spectral energy density of the radiation field that a transition will occur. For stimulated absorption, the absorbed photon has an energy of $h v_{0}=E_{u}-E_{l}$, where $v_{0}$ is the frequency of the transition $[\mathrm{Hz}]$ and $h$ is the Planck constant $\left[6.626 \times 10^{-34} \mathrm{~J} \mathrm{~Hz}^{-1}\right]$.

Stimulated emission is controlled by the Einstein coefficient $B_{u l}\left[\mathrm{~J} \mathrm{~s}^{-1} \mathrm{~m}^{2} \mathrm{sr} \mathrm{molec}^{-1}\right]$, giving the probability per second per spectral energy density of the radiation field that a transition will take place from the upper level to the lower level.

Spontaneous emission is described by the Einstein coefficient $A_{u l}\left[\mathrm{~s}^{-1} \mathrm{molec}^{-1}\right]$ which is the probability per second that a transition will spontaneously occur from the upper level to the lower level. For stimulated and spontaneous emission, the emitted photon has an energy of $h v_{0}=E_{u}-E_{l}$ and the inducing radiation field for stimulated emission must have a frequency close to $v_{0}$.

In addition to these three radiative transitions, transitions between the lines can also be induced by collisions between atoms. In this case, the transitions between the lower and upper energy levels, and the upper and lower levels are represented by collisional rates $C_{l u}$ and $C_{u l}$, respectively, which describe the probability per second of a transition occurring as a result of a collision.

The population of individual levels must be solved using a system of equations (Yamada et al. 2018):

$\frac{\mathrm{d} n_{i}}{\mathrm{~d} t}=0=\sum_{j>i}\left[n_{j} A_{j i}-\left(n_{i} B_{i j}-n_{j} B_{j i}\right) \bar{J}_{j i}\right]-\sum_{j<i}\left[n_{i} A_{i j}-\left(n_{j} B_{j i}-n_{i} B_{i j}\right) \bar{J}_{i j}\right]+\sum_{j}\left(n_{j} C_{j i}-n_{i} C_{i j}\right)$

where $n_{i}$ is the population of energy level $i, t$ is time [s], and $\bar{J}_{i j}$ is the integrated mean intensity:

$$
\bar{J}_{i j}=\frac{1}{4 \pi} \int_{0}^{\infty} \mathrm{d} \nu \int_{0}^{4 \pi} I_{\nu} \phi_{i j, \nu} \mathrm{d} \Omega
$$

where $\phi_{i j, v}$ is the frequency dependent line emission profile. Equation 2.2 is called the statistical equilibrium equation (Rybicki and Hummer 1991).

\subsection{Optical depth}

When an incoming ray of light moves through a medium (Blundell and Blundell 2009), the intensity of the electromagnetic wave is changed from its initial spectral intensity, $I_{v, 0}$, to its outgoing spectral intensity, $I_{v}$, in units of $\left[\mathrm{J} \mathrm{s}^{-1} \mathrm{~m}^{-2} \mathrm{sr}^{-1} \mathrm{~Hz}^{-1}\right]$. The subscript ${ }_{v}$ denotes the frequency dependence of the terms. The amount of change in intensity depends upon the mass density of absorbing molecules in the material, $\rho_{a}\left[\mathrm{~kg} / \mathrm{m}^{3}\right]$; and the composition of the medium. The composition of the medium is represented by a parameter 
called the opacity, $\kappa_{v}\left[\mathrm{~m}^{2} / \mathrm{kg}\right]$, which describes how efficiently radiation is absorbed by the material. It is also frequency dependent.

In a homogeneous medium with absorption only, the amount of intensity lost in the medium is represented by:

$$
d I_{v}=-\kappa_{v} \rho_{a} I_{v} d s
$$

where $d s$ is some spatial element of the medium. Integrating Equation 2.4 gives:

$$
I_{v}=I_{v, 0} \exp \left(-\kappa_{v} \rho_{a} s\right)
$$

which describes an exponential decay of the intensity with distance. This result is commonly known as the Beer-Lambert law. The mean free path, $l$, can be defined as the distance for the intensity to decrease by $1 / \mathrm{e}$ or:

$$
l=\frac{1}{\kappa_{v} \rho_{a}}
$$

in the homogeneous medium.

Optical depth, $\tau_{v}$, combines opacity and density into one parameter and defines the ability of the material to block light:

$$
\tau_{v}=\int_{0}^{s_{o}} \kappa_{v} \rho_{a} \mathrm{~d} s=\int_{0}^{s_{o}} \alpha_{v}(s) \mathrm{d} s
$$

where $s_{o}$ is the length of the medium and $\alpha_{v}(s)$ is the absorption coefficient $\left[\mathrm{m}^{-1}\right]$. For a non-homogeneous medium, $\kappa_{v}, \rho_{a}$ and $\alpha_{v}$ change within the material.

Notice that the optical depth is a function of the opacity, so that as the opacity increases, so does the optical depth. However, a material can have a large opacity but a small optical depth, depending on the magnitude of the density.

There are a few special cases for $\tau_{v}$. When $\tau_{v} \ll 1$, the medium is said to be optically thin and essentially transparent as the medium does not absorb the incoming intensity. In this case, the radiation mean free path is far greater than the length of the medium and radiation can propagate without any absorption. Any changes in extinction are proportional to the amount of material in the medium, so in the optically thin regime, extinction is proportional to the density of the absorbers.

When $\tau_{v}=1$, the mean free path is the same length as the medium. Generally, we can see about one mean free path, or one optical depth, into a material.

In the other extreme, where $\tau_{v} » 1$, the medium is completely opaque. The medium is far greater in length than the mean free path and only radiation from the surface can be seen. The proportionality to density is lost in the optically thick case, and the medium now acts like a blackbody, radiating with the intensity found at the surface.

A blackbody is an ideal object, absorbing and emitting with $100 \%$ efficiency. Emissivity is the ratio of actual emission to blackbody emission. Blackbodies have an emissivity equal to 1 and non-blackbodies have an emissivity less than 1. A similar definition exists for absorptivity, and Kirchoff's law states that the emissivity of a medium is equal to its absorptivity at a particular frequency. 


\subsection{Radiative transfer equation}

The optical depth is the fundamental property regarding radiative transfer. Radiative transfer describes the propagation of radiation through a medium (Chance and Martin 2017). The change in intensity of radiation as it moves through a medium is modulated by absorption, which reduces the intensity, emission, which enhances the intensity, and scattering processes, which can reduce and enhance the intensity. The radiative transfer equation takes the form:

$$
\frac{\mathrm{d} I_{v}(s)}{\mathrm{d} s}=j_{v}(s)-\alpha_{v}(s) I_{v}(s)
$$

where $j_{v}(s)$ is the spectral emissivity with units of $\left[\mathrm{J} \mathrm{s}^{-1} \mathrm{~m}^{-3} \mathrm{sr}^{-1} \mathrm{~Hz}^{-1}\right]$ (Janssen 1994). All of these parameters are functions of frequency, $v$, and the distance that the radiation has moved through the medium, $s$. The equation describes how the intensity which enters the medium is changed by the medium (left hand side) through gains as a result of emission from local contributions $\left(j_{v}(s)\right)$ and losses from self-absorption $\left(\alpha_{v}(s) I_{v}(s)\right)$. In the case where emission is negligible, $j_{v}(s)=0$, the Beer-Lambert Law in Equation 2.5 can be retrieved from the radiative transfer equation.

The spectral emissivity and the absorption coefficient depend upon the occupation of molecular energy levels:

$$
\begin{gathered}
j_{v}(s) \equiv \frac{\rho}{4 \pi} f_{u} A_{u l} h v_{0} \psi\left(v, v_{0}\right) \\
\alpha_{v}(s) \equiv \frac{\rho}{4 \pi}\left(f_{l} B_{l u}-f_{u} B_{u l}\right) h v_{0} \psi\left(v, v_{0}\right)
\end{gathered}
$$

where $f_{u}$ is the fraction of molecules in the upper energy level and $f_{l}$ is the fraction of molecules in the lower energy level, $\rho$ is the molecular number density $\left[\mathrm{m}^{-3}\right], v$ is the frequency and $\psi$ is a normalised line profile so that $\int_{0}^{\infty} \psi\left(v, v_{0}\right) \mathrm{d} v \equiv 1$. The normalised line profiles for $j_{v}(s)$ and $\alpha_{v}(s)$ are assumed to be the same (complete frequency redistribution). The spectral emissivity and the absorption coefficient are functions of the three Einstein coefficients from Section 2.2 and they can be expressed in terms of $g_{u}$ and $g_{l}$, the statistical weights of each energy level, using the equations:

$$
\frac{B_{l u}}{B_{u l}}=\frac{g_{u}}{g_{l}}
$$

and:

$$
A_{u l}=\frac{2 h v^{3}}{c^{2}} B_{u l}
$$

where $c$ is the speed of light in a vacuum.

A source function, $S_{v}(s)$, can be defined as $S_{v}(s) \equiv \frac{j_{v}}{\alpha_{v}}$ so that Equation 2.8 can be rewritten as:

$$
\frac{\mathrm{d} I_{v}(s)}{\mathrm{d} \tau_{v}}=S_{v}(s)-I_{v}(s)
$$

and the differential is now in terms of $\mathrm{d} \tau$ since $\mathrm{d} \tau_{v}=\alpha_{\nu}(s) \mathrm{d} s$ following from Equation 2.7. 
Using the derivation in Appendix A, the radiative transfer in Equation 2.8 can be expressed as:

$$
I_{v}=I_{v, 0} \exp \left(-\tau_{v}\left(s_{o}\right)\right)+\int_{0}^{s_{o}} S_{v} \exp \left(-\tau_{v}(s)\right) \alpha_{v}(s) d s
$$

The intensity at the observer, $I_{\nu}$, is the sum of the attenuated intensity from the source, $I_{v, 0}$, and all the contributions from the material along the line of sight with a length $s_{o}$ (second part of the right hand side of the equation).

With Equations 2.9 and 2.10, we can express the source function as:

$$
S_{v}=\frac{2 h v^{3}}{c^{2}} \frac{1}{\frac{g_{u}}{g_{l}} \frac{f_{l}}{f_{u}}-1}
$$

The population of the energy levels is therefore fundamentally important in order to solve the radiative transfer equation.

\subsection{LTE vs. non-LTE}

A system is in Local Thermodynamic Equilibrium (LTE, hereafter) when the energy level distribution is determined by the collisional terms $\left(C_{l u}\right.$ and $\left.C_{u l}\right)$ in the statistical equilibrium equation (Equation 2.2). A local region can then equilibrate via collisions and the region can be described by a single temperature (Chance and Martin 2017). This may occur at a specific altitude in an atmosphere or coma. In LTE conditions, temperature can still vary globally within the system but at a specific location, thermodynamic equilibrium can be assumed (Blundell and Blundell 2009).

The assumption of LTE allows the Boltzmann distribution to be applied to the system to find the fraction occupying an energy level, $i$, at a temperature, $T$ :

$$
f_{i}=\frac{g_{i} \exp \left(\frac{-E_{i}}{k T}\right)}{Z(T)}
$$

where $Z(T)$ is the partition function:

$$
Z(T)=\sum_{i} g_{i} \exp \left(\frac{-E_{i}}{k T}\right)
$$

which describes how populations are distributed among the different levels and energies (Chance and Martin 2017).

In LTE, the source function can be expressed as:

$$
S_{v}=\frac{2 h v^{3}}{c^{2}} \frac{1}{\exp \left(\frac{h v}{k T}\right)-1}
$$

which is simply a Planck function, $B_{v}(T)$, where $k$ is the Boltzmann constant and $T$ is the blackbody temperature.

At low microwave frequencies, and thus long wavelengths $(\lambda)$, the Rayleigh-Jeans approximation can be used (Janssen 1994), whereby $h v \ll k T$, so that the Planck equation reduces to: 


$$
B_{v}(T)=\frac{2 v^{2} k T}{c^{2}}
$$

or:

$$
B_{\lambda}(T)=\frac{2 k c T}{\lambda^{4}}
$$

because in the Rayleigh-Jeans limit:

$$
\frac{1}{\exp \left(\frac{h v}{k T}\right)-1}=\frac{k T}{h v}
$$

Brightness temperature, $T_{b}$ is defined as the blackbody temperature required in order to produce a measured power and can be expressed as:

$$
T_{b}(v)=\frac{c^{2}}{2 k v^{2}} I_{v}
$$

which allows the radiative transfer equation to be written in terms of the brightness temperature:

$$
T_{b}(v)=T_{b, o}(v) \exp \left(-\tau\left(s_{o}\right)\right)+\int_{0}^{s_{o}} T(s) \exp \left(-\tau_{v}(s)\right) \alpha_{v}(s) d s
$$

This form of the equation is commonly used in LTE microwave remote sensing and in applications for analysing the MIRO dataset.

At low densities however, especially those expected for cometary comae, the collisional frequency of molecules is not high enough to keep the LTE conditions and other processes have to be taken into account. In this case we speak of the breakdown of LTE and must instead use the non-Local Thermodynamic Equilibrium (non-LTE, hereafter) regime.

When collisions no longer dominate, Equation 2.16 can not be used in non-LTE and instead, we must account for all the detailed processes populating and depopulating the levels which are expressed in the statistical equilibrium equation (Rybicki and Hummer 1991). Clearly, having to use Equation 2.2 for each level makes non-LTE calculations far more computationally intensive and time consuming than simply using the LTE approximation. In LTE, the collisional terms dominated so that Equation 2.2 greatly reduced in complexity.

Within cometary comae, it is vitally important to consider the radiative transfer problem in non-LTE due to the tenuous nature of the atmospheres (Yamada et al. 2018). Nevertheless, for high production comets $\left(>1 \times 10^{27} \mathrm{molec} / \mathrm{s}\right)$, the collisional terms can sometimes dominate up to $50-100 \mathrm{~km}$, in which case using LTE conditions is justified. In addition, the choice between using LTE or non-LTE can depend on the science question: if considering just the line area, LTE may be valid within some uncertainties (as most of the line is formed inside the LTE region), but if the information provided by the line shape is investigated, then non-LTE calculations have to be considered (such that the region around the line core is accurately considered).

Scattering processes are also important in the radiative transfer equation but for the microwave regime which we are interested in, scattering can be ignored. Figure 2.2 is 


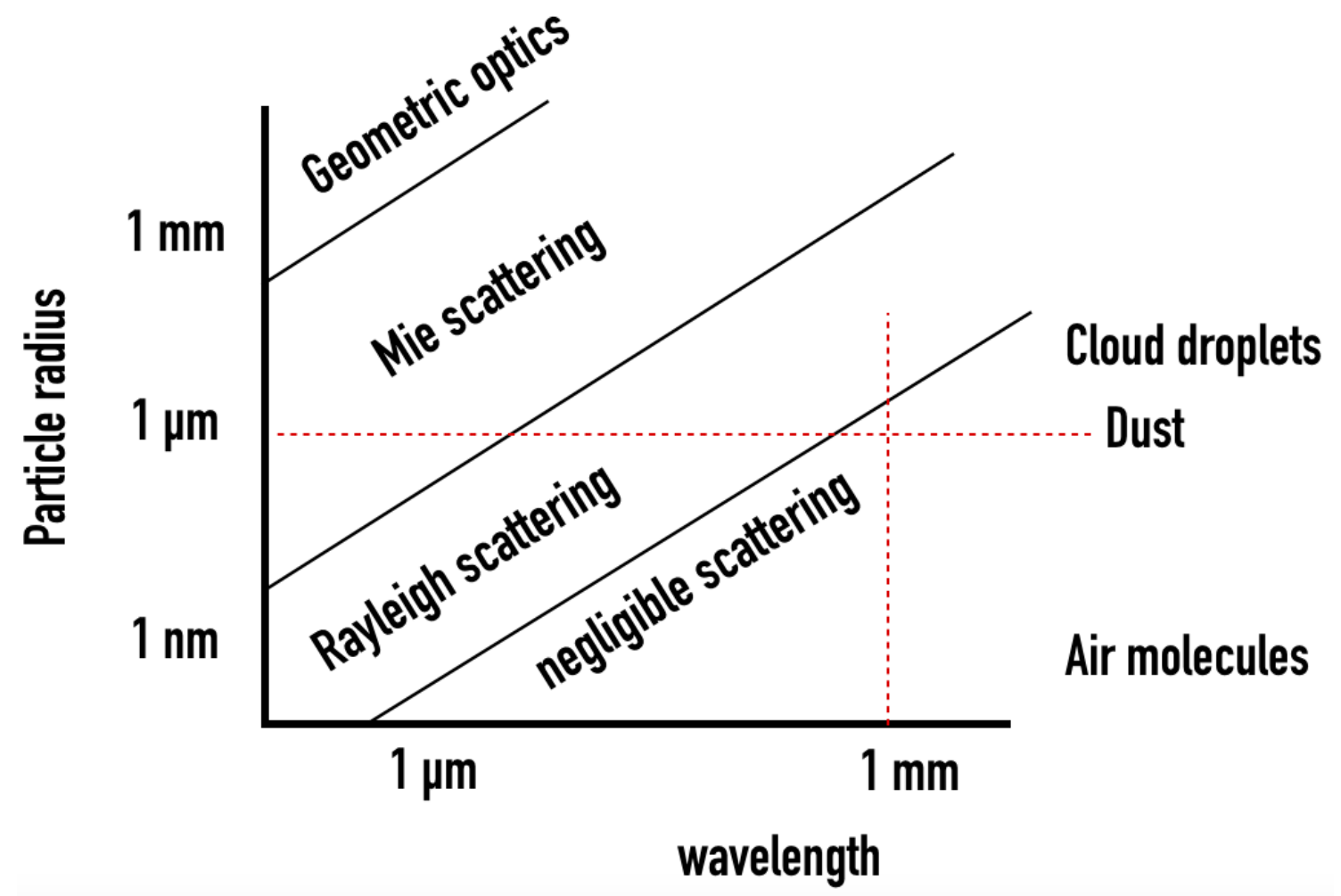

Figure 2.2: Schematic representation depicting where different scattering regimes dominate at different wavelengths and for different particle radii. The red lines approximately indicate the wavelength of MIRO and the size of dust particles. Adapted from Chance and Martin (2017)

reproduced from Chance and Martin (2017) and shows where different scattering regimes dominate for various wavelengths and particle sizes. The MIRO instrument takes measurements at millimetre and submillimetre wavelengths where scattering is mostly negligible for the small dust particles expected in cometary comae.

\subsection{Inversion methods}

A forward model can be thought of as using a set of parameters, $x$, in a function, $F(x)$, to predict a set of measurements, $y$. This can be written as:

$$
y=F(x)
$$

An inverse problem, as the name suggests, is the reverse procedure of the forward model: using the measurements to predict the initial parameters. The inverse problem takes the form:

$$
x=F^{-1}(y)
$$

The function $F$ is often non-linear and hence requires a linear approximation to simplify the problem. This approximation is called quadrature (Twomey 2013) and $F$ is recast 
as the weighting function $K$. Equations 2.24 and 2.25 hence become:

$$
\begin{gathered}
y=K x \\
x=K^{-1} y
\end{gathered}
$$

An inverse problem is said to be well-posed if a solution exists, which is unique, and depends continuously on the data. In reality, inverse problems are rarely well-posed.

In the inverse problem which will occur later in this work, the parameters which we want to find, our $x$, will be temperature, velocity and density values (discretised in the radial direction), while the measurements, our $y$, will be the intensity of water spectral lines. As will be discussed later, the desired parameters cannot all be uniquely determined. The source of the non-uniqueness arises due to the nature of the radiative transfer equation which relates the desired parameters, and due to measurement uncertainty.

In addition, the measurements may not depend continuously on the parameters. This problem is especially prevalent in the optically thick case where changes in the density do not always have an effect on the intensity of the spectra. As a result of these issues, our problem is said to be ill-posed (Twomey 2013).

The non-linearity of the problem must be carefully considered because it may, under certain conditions, also lead to instability in the inversion. Simply put, this could occur when changes in the parameter space do not produce changes in measurement space.

So, how can these problems be overcome?

We can rewrite the inverse problem in terms of the least squared solution, where the aim is to minimise the equation:

$$
\min \|K x-y\|^{2}
$$

In constrained least squares, an additional, if arbitrary, term is added to the cost function and Equation 2.28 becomes:

$$
\min \|K x-y\|^{2}+\|\gamma H\|^{2}
$$

where $\gamma$ can take any finite value and $H$ is a smoothing matrix, typically the identity matrix or a near-diagonal matrix. Adding this additional constraint is called regularization. This additional term effectively limits the solutions to a subset of all solutions, thereby reducing the degeneracy of the problem and also helping to stabilise the inversion.

Regularization can be performed in a different way that also incorporates correlation among the parameters in the a priori information. In can take the form:

$$
\min \left\|(K x-y) S_{e}(K x-y)+\left(x-x_{a}\right) S_{a}\left(x-x_{a}\right)\right\|^{2}
$$

where $S_{e}$ and $S_{a}$ are measurement and a priori covariance matrices, and $x_{a}$ is some a priori information on the parameter space. The covariance matrices can add smoothness to the solutions, and thus remove any wild and discontinuous solutions. Furthermore, solutions can now be limited to stay close to the a priori. This type of regularisation is typically used in optimal estimation methods of inversion (Rodgers 2000). Optimal Estimation is an iterative process which takes the form of:

$$
x_{i+1}=x_{a}+S_{a} K^{T}\left(K S_{a} K^{T}+S_{e}\right)^{-1}\left(y-F\left(x_{i}\right)+K\left(x_{i}-x_{a}\right)\right)
$$


where $i$ is the iteration number and $T$ denotes a transposed matrix.

It is challenging to apply Equation 2.31 to radiative transfer problems for comets. Firstly, there is little a priori knowledge regarding cometary comae, especially the inner regions within $100 \mathrm{~km}$ from the nucleus. Creating a suitable $x_{a}$ is therefore difficult. In addition, the covariance in the coma is unknown so $S_{a}$ acts like a free parameter which would otherwise be fixed if this was an Earth atmosphere inverse problem. In Chapter 6, I will describe out the Optimal Estimation inverse method can be applied to measurements made from $67 \mathrm{P}$ and how these issues can be dealt with. 


\section{Paper I - Spatially resolved evolution of the local $\mathrm{H}_{2} \mathrm{O}$ production rates of comet 67P/Churyumov-Gerasimenko from the MIRO instrument on Rosetta}

This chapter has been published in A\&A as the journal article: Marshall, D. W., et al. "Spatially resolved evolution of the local $\mathrm{H} 2 \mathrm{O}$ production rates of comet 67P/ChuryumovGerasimenko from the MIRO instrument on Rosetta." Astronomy \& Astrophysics 603 A87 2017. Reproduced with permsission (c) ESO

\subsection{Summary}

Using spectroscopic and continuum data measured by the MIRO instrument on board Rosetta of comet 67P/Churyumov-Gerasimenko, it is possible to derive and track the change in the water production rate, to learn how the outgassing evolves with heliocentric distance. The MIRO data are well suited to investigate the evolution of 67P, in unprecedented spatial and temporal detail. To obtain estimates of the local effective Haser production rates we developed an efficient and reliable retrieval approach with precalculated lookup tables. We employed line area ratios $\left(\mathrm{H}_{2}{ }^{16} \mathrm{O} / \mathrm{H}_{2}{ }^{18} \mathrm{O}\right)$ from pure nadir observations as the key variable, along with the Doppler shift velocity, and continuum temperature. This method was applied to the MIRO data from August 2014 until April 2016. Perihelion occurred on August 13, 2015 when the comet was 1.24 AU from the Sun. From the start of the period until seven days before perihelion the water production rates increased by an order of magnitude, and the derived maximum $(1.42 \pm 0.51) \times 10^{28}$ is for August 29, 2015. The data also indicate that there is an offset in the peak outgassing, occurring 2246 days after perihelion, depending which kind of fitting is used. In the pre-perihelion, the production rate changes with heliocentric distance like $r_{h}^{-3.8 \pm 0.2}$, while post-perihelion the dependence is $r_{h}^{-4.3 \pm 0.2}$. In addition, this method provides well sampled dataset to determine the spatial distribution of the outgassing versus heliocentric distance. The time evolution is definitely not uniform across the surface. During the approach toward the perihelion the surface temperature on the southern hemisphere increases rapidly and so does the sublimation rate with an exponent of $\sim-6$ pre- and post-perihelion. We also present more detailed regional variation in the outgassing rate, which demonstrates that 
the highest derived production rates originate from the Wosret, Neith and Bes regions during perihelion.

\subsection{Introduction}

The water production rate of comet 67P/Churyumov-Gerasimenko has been measured by a variety of instruments on board Rosetta. In June 2014, MIRO (Microwave Instrument for the Rosetta Orbiter; see Section 3.3 for more details) measured the water production rate at a heliocentric distance of $3.9 \mathrm{AU}$ to be $10^{25}$ molecules/second, increasing to $4 \times 10^{25} \mathrm{molec} / \mathrm{s}$ by late August (Gulkis et al. 2015). Only Bockelee-Morvan et al. (2010) and de Val-Borro et al. (2014) have determined water outgassing rates from comets at larger heliocentric distances. Also in August 2014, Lee et al. (2015) demonstrated that the outgassing across the surface can vary by a factor of 30 from $0.1 \times 10^{25} \mathrm{molec} / \mathrm{s} / \mathrm{sr}$ to $3.0 \times 10^{25} \mathrm{molec} / \mathrm{s} / \mathrm{sr}$. In the next month, MIRO estimated the production of the water isotopologue $\mathrm{H}_{2}{ }^{16} \mathrm{O}$ as $(4.9 \pm 2.5) \times 10^{25} \mathrm{molec} / \mathrm{s}$ on September 7 at $3.41 \mathrm{AU}$ from the Sun (Biver et al. 2015). The ROSINA-COPS (Rosetta Orbiter Spectrometer for Ion and Neutral Analysis - COmetary Pressure Sensor) instrument found production rates in the range of $8.7 \times 10^{25}-1.1 \times 10^{26}$ molec/s between August and November 2014, which then increased by a factor of 2 between November and January 2015 (Bieler et al. 2015). Fougere et al. (2016) present results from ROSINA-DFMS (Double Focusing Mass Spectrometer), which show the outgassing increasing from $<10^{26} \mathrm{molec} / \mathrm{s}$ at $3.5 \mathrm{AU}$ (August 2014) to $>10^{27} \mathrm{molec} / \mathrm{s}$ at $1.5 \mathrm{AU}$ from the Sun (May 2015). A mean production rate of $8 \times 10^{25} \mathrm{molec} / \mathrm{s}$ agrees with results from VIRTIS-H (Visible InfraRed Thermal Imaging Spectrometer) between November 2014 and January 2015 (Bockelée-Morvan et al. 2015), and data in April 2015 from VIRTIS-M suggest that the rate increases to about $10^{27} \mathrm{molec} / \mathrm{s}$ (Migliorini et al. 2016). The ROSINA spectrometer is an in situ instrument unlike MIRO and VIRTIS, which are remote sounding instruments.

Together, these data demonstrate the increasing activity of the comet towards perihelion. In this work, we present the long-term evolution of the water production rate of comet 67P from MIRO observations as it approaches and recedes from the Sun, between August 2014 and April 2016, with the aim to spatially resolve the water outgassing. Through this, we can analyse the behaviour of the comet with respect to heliocentric distance and the change in activity across the surface. We use a lookup table method for the inversion of line areas into column densities and production rates and hence derive local effective Haser production rates of water (defined in Section 3.4.2) from the line area ratios of $\mathrm{H}_{2}{ }^{16} \mathrm{O}$ and $\mathrm{H}_{2}{ }^{18} \mathrm{O}$. This approach is simple, producing results that are consistent with other measurements from Rosetta instruments along with ground-based observations. Our methodology which enables large quantities of data to be inverted quickly and reliably, allowing the production rates from the 21-month MIRO dataset to be calculated in minutes and enabling us to study the outgassing evolution with good spatial resolution. With the spatially resolved data, we can study the processes driving the gas activity and investigate how the shape and illumination modulate this behaviour. The lookup table method is fully described in Section 3.4 along with a thermal sublimation model to help interpret the results, which are given in Section 3.5. The work is then summarised in Section 3.6. The next section gives a brief description of MIRO, the observations and the 
shape model.

\subsection{MIRO observations}

The Microwave Instrument for the Rosetta Orbiter (MIRO) is a small millimetre/submillimetre spectrometer with a $30 \mathrm{~cm}$ offset parabolic reflector telescope and two heterodyne receivers. The millimetre receiver operates at a centre-band frequency of $188 \mathrm{GHz}$ (1.6 mm wavelength) while the sub-millimetre receiver is tuned at $562 \mathrm{GHz}(0.5 \mathrm{~mm})$. Each frequency band contains a single broadband channel for the measurement of near surface temperatures. The continuum data have been previously analysed by Schloerb et al. (2015) and for polar night conditions by Choukroun et al. (2015). For our work, we are interested in the data from the Chirp Transform Spectrometer (CTS; Hartogh and Hartmann (1990)), which is connected to the sub-millimetre receiver.

The CTS has 4096 channels with a spectral resolution of $44 \mathrm{kHz}$, corresponding to a resolution of $v / \Delta v=10^{7}$ for the sub-mm channel. It can observe three isotopologues of water $\left(\mathrm{H}_{2}{ }^{16} \mathrm{O}, \mathrm{H}_{2}{ }^{17} \mathrm{O}\right.$, and $\left.\mathrm{H}_{2}{ }^{18} \mathrm{O}\right)$, three methanol lines, carbon monoxide and ammonia at frequencies of $547 \mathrm{GHz}$ to $580 \mathrm{GHz}$. A high spectral resolution is necessary for observing the narrow, Doppler broadened spectral lines of the low temperature, low pressure coma. MIRO collects spectral data every 30 seconds in a frequency switched mode. For the full technical details of the instrument, see Gulkis et al. (2007). We focus on the MIRO data collected from 3.62 AU in August 2014 to 1.24 AU at perihelion on August 13, 2015, and on the outbound leg to 2.02 AU in April 2016. In this time interval, the nucleus of the coma was fully resolved during the nadir observations.

We use a digital shape model of the comet (SHAP5 v1.2, Preusker et al. (2015)) with 200,000 triangular facets and the SPICE software provided by the Jet Propulsion Laboratory (JPL; Acton (1996)) to calculate the intersection geometry. With the shape model, the SPICE software and the beam pointing provided by the project, we can track the movement of the spacecraft and the comet to determine the facet-averaged position of the MIRO beam, solar illumination, solar zenith angle and viewing angle.

\subsection{Method}

We employed an efficient method using lookup tables to determine the water production rate of comet 67P. The basic idea of lookup table inversion is straight forward. We precalculated a table of water production rate, $\mathrm{Q}$, versus line area via a forward radiative transfer model (Section 3.4.2). Once the tables are calculated, the radiative transfer calculations are no longer required, and the measured line area ratios entered into a lookup table obtain the corresponding water production rate. In the application to the MIRO data, we used additional information to provide more unique mapping (using more than one table), such as the observed Doppler shift and continuum temperature. It is necessary to use more than one lookup table to test the sensitivity of the production rate measurements to each variable (Doppler shift and continuum temperature). We considered only nadir absorption spectra in this work and treated the line area ratio, $\mathrm{H}_{2}{ }^{16} \mathrm{O} / \mathrm{H}_{2}{ }^{18} \mathrm{O}$, as the fundamental variable. Below, the details of the methodology and measurement selection are provided. 
Our approach is different from work by Biver et al. (2016), who have tracked the change in the global water production of $67 \mathrm{P}$ with heliocentric distance, as well as other molecules. In this work, we looked exclusively at nadir observations of the comet to derive local production rates and we neglected any geometrical effects. We then used the local production rates to track the evolution of the outgassing in each region as function of heliocentric distance.

\subsubsection{Water absorption lines}

In this analysis we focus only on the nadir absorption lines for the two water isotopes, $\mathrm{H}_{2}{ }^{16} \mathrm{O}$ and $\mathrm{H}_{2}{ }^{18} \mathrm{O}$ owing to their different opacities, and because they are routinely detected in the time period of interest (August 2014 to April 2016). Examples of these absorption lines can be seen in Gulkis et al. (2015), Biver et al. (2015), and Lee et al. (2015) (Figure 3), where the latter makes a distinction between the appearance of nadir, off-nadir, and limb viewing spectra. Processing the spectra involves several steps. First, we excluded spectra with low signal to noise (the line area must be greater than $2 \mathrm{~K} \mathrm{~km} / \mathrm{s}$ for both isotopes), and then we excluded any emission lines, including limb and partial limb spectra. The $2 \mathrm{~K} \mathrm{~km} / \mathrm{s}$ value for the noise limit was determined by calculating the average line area for continuum emission spectra without a line feature and with only random noise. The observations were averaged into 30-minute time intervals to reduce random noise; for example, in April 2016, the noise level decreased from $1 \mathrm{~K} \mathrm{~km} / \mathrm{s}$ to $0.14 \mathrm{~K} \mathrm{~km} / \mathrm{s}$ as we increased the time interval for averaging the spectra from 30 seconds to 30 minutes. Subsequently, we smoothed the spectra with an 8 point box car filter. This smoothing has a minimal effect on the determination of the spectral line areas $(<0.1 \%)$, but allows for a better determination of the Doppler shift by the automatic lookup method. The Doppler shift was simply calculated from the velocity shift of the minimum value of an absorption line.

We found the line area by integrating in the region where the line forms, between -2 and $1.1 \mathrm{~km} / \mathrm{s}$, which is wide enough to ensure that broadened lines are not cut off even for the wide $\mathrm{H}_{2}{ }^{16} \mathrm{O}$ lines in the case of high gas production. The estimates for the line area error come from the random noise in the line wings (greater than $1.2 \mathrm{~km} / \mathrm{s}$ ) and calculated with the equation,

$$
\sigma=\sqrt{N} \Delta v \sigma_{T},
$$

where $\mathrm{N}$ is the number of data points between -2 and $1.1 \mathrm{~km} / \mathrm{s}, \Delta \mathrm{v}$ is the average velocity interval between each point in the spectrum, and $\sigma_{T}$ is the standard deviation of the antenna temperature points in the wings $(>1.2 \mathrm{~km} / \mathrm{s})$

In Figure 3.1, the time evolution of the nadir line areas for the two isotopes are presented. For both isotopologues, the activity increases towards perihelion owing to the increase in outgassing, and decreases after perihelion, but with a delay (discussed later). The line area uncertainties due to random noise are small, typically $0.2 \mathrm{~K} \mathrm{~km} / \mathrm{s}$ for both isotopologues with measurements in the range of $13-385 \mathrm{~K} \mathrm{~km} / \mathrm{s}$ for $\mathrm{H}_{2}{ }^{16} \mathrm{O}$ and from 2 $-135 \mathrm{~K} \mathrm{~km} / \mathrm{s}$ for $\mathrm{H}_{2}{ }^{18} \mathrm{O}$ (see Figure 3.1).

The line area ratio is a sensitive tracer of opacity and column density in the coma (Figure 3.1). Providing that the optical depth is less than unity, the line area is linearly related to the column density. However, as the column density increases further, line area 

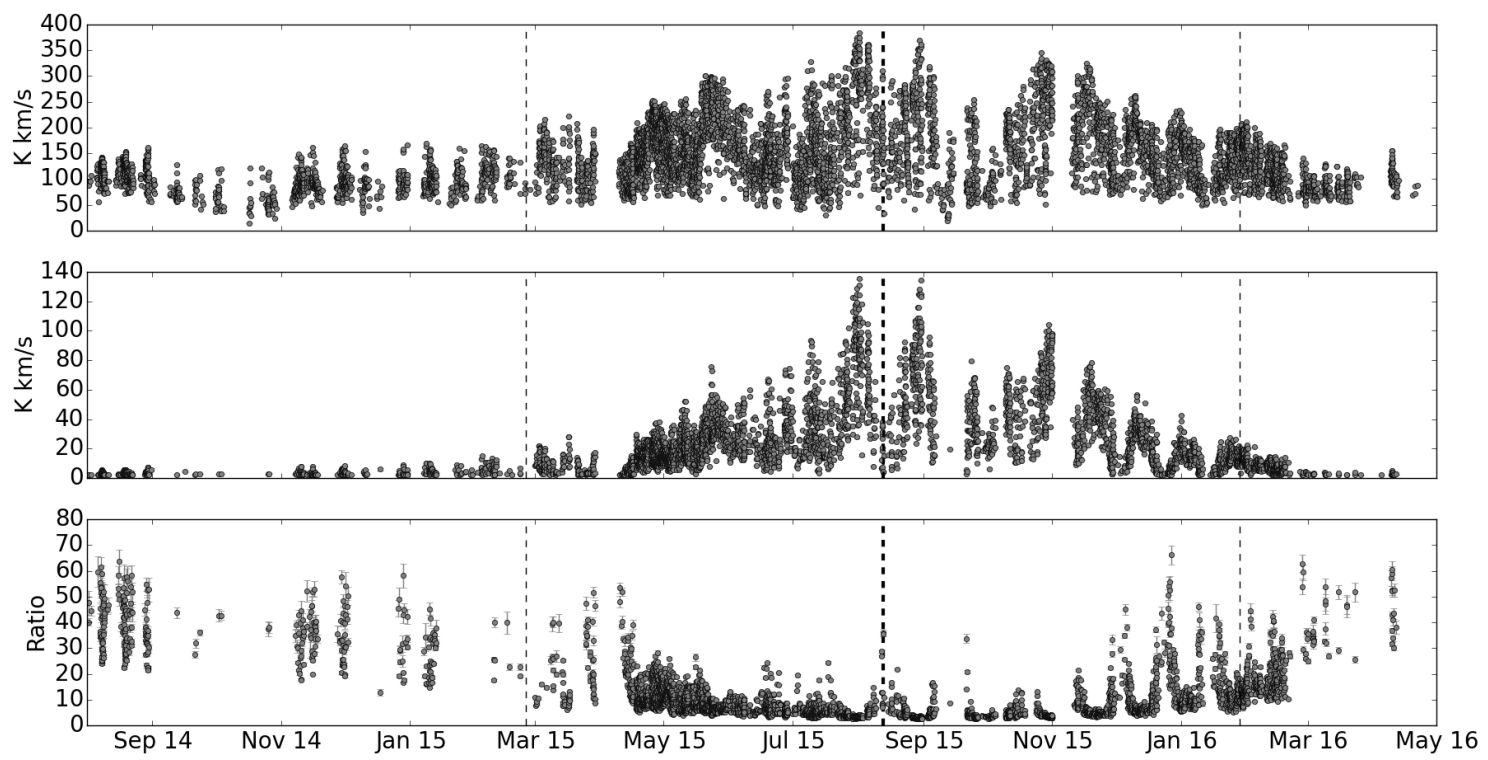

Figure 3.1: Time evolution of the line area of $\mathrm{H}_{2}{ }^{16} \mathrm{O}$ (top panel), $\mathrm{H}_{2}{ }^{18} \mathrm{O}$ (middle panel), and the $\mathrm{H}_{2}{ }^{16} \mathrm{O} / \mathrm{H}_{2}{ }^{18} \mathrm{O}$ line area ratio (bottom panel) from August 2014 to April 2016. The thick dashed line represents perihelion, which occurred on August 13, 2015, and the thin dashed lines represent when 67P was 1 AU from closest approach, pre- and postperihelion.

growth loses this linearity and the dependence becomes $\sqrt{\ln N}$, where $\mathrm{N}$ is the number density (Goldsmith and Langer 1999). The line is said to be saturated, as the amplitude does not change with increasing column density. The $\mathrm{H}_{2}{ }^{16} \mathrm{O}$ and $\mathrm{H}_{2}{ }^{18} \mathrm{O}$ lines observed by MIRO follow this pattern. Because these two lines have different opacities, the $\mathrm{H}_{2}{ }^{16} \mathrm{O}$ line is always saturated with an opacity $>10$ (Gulkis et al. 2015) whereas the $\mathrm{H}_{2}{ }^{18} \mathrm{O}$ line starts out with a small amplitude and is optically thin (opacity $<0.1$ ) but then grows with the column density. By combining them in a ratio we produce a more favourable line growth curve with greater sensitivity as we approach the saturated part of the growth curve.

In Figure 3.1, the ratio indicates that in August 2014 the $\mathrm{H}_{2}{ }^{16} \mathrm{O}$ is optically thick as the highest values are around 60-70, much lower than the isotopic ratio of 498.7 (terrestrial value). The ratios further decrease towards the perihelion as even the $\mathrm{H}_{2}{ }^{18} \mathrm{O}$ line becomes optically thick, and the ratio reaches values as low as 2.5. About 90 days after perihelion the ratio grows again significantly as the water production goes down.

\subsubsection{Generating the lookup tables}

We performed the lookup table calculations with a non-LTE forward model. For radiative transfer in comets, two different computational methods are typically used: a locally approximated escape probability approach (Bockelée-Morvan 1987, Litvak and Kuiper 1982) and a direct ray transfer Monte Carlo approach (Hogerheijde and van der Tak 2000). Both methods have been found to produce similar results numerically (Zakharov et al. 2007) and in comparison to observations (Hartogh et al. 2010b, de Val-Borro et al. 2010). Building a lookup table involves hundreds of calculations, which with the Monte Carlo approach, would take weeks to compute. We instead used the escape probaility approach 
following Lee et al. (2015) (see references therein for detailed description and accuracy), which required substantially less computational time.

The atmospheric inputs are defined radially over $500 \mathrm{~km}$ from the nucleus surface with grid points (integer index i) calculated at

$$
\frac{R_{d} C^{i}+R_{d} C^{i+1}}{2}
$$

where $\mathrm{R}_{d}$ is the radius of the comet, taken as $2 \mathrm{~km}$, multiplied by a scaling constant, $\mathrm{C}=1.02$, to define 278 logarithmic grid points over $500 \mathrm{~km}$. The temperature profile follows an adiabatic expansion. The inputs are the surface temperature parameter, $\mathrm{T}_{s}$, and the terminal temperature, which is estimated from the line cores of the $\mathrm{H}_{2}{ }^{16} \mathrm{O}$ lines observed by MIRO and fixed at $30 \mathrm{~K}$. The velocity profile is modelled by a hyperbolic tangent function and increases from zero to the terminal expansion velocity, $\mathrm{v}_{\text {exp }}$, as described in Lee et al. (2015). The radial molecular number density profile is dictated by the Haser model (Haser 1957) which depends on the production rate, $\mathrm{Q}$, expansion velocity profile and $\beta$ parameter, which is also taken from Lee et al. (2015). The $\beta$ parameter is the photodissociation rate with a value of $1.4 \times 10^{-5} \mathrm{~s}^{-1}$ at a distance of $1 \mathrm{AU}$, but it is not critical for deriving Q. In addition, we assume the $\mathrm{H}_{2}{ }^{16} \mathrm{O} / \mathrm{H}_{2}{ }^{18} \mathrm{O}$ ratio and the ortho-para ratio to be 500 and 3, respectively, in our forward calculations, as in previous works. In total, three atmospheric parameters are varied to generate two lookup tables for the water production rate: the surface temperature, $\mathrm{T}_{s}(\mathrm{~K})$; the terminal expansion velocity, $\mathrm{v}_{\exp }(\mathrm{km} / \mathrm{s})$; and $\mathrm{Q}$ (molec/s).

In the first table, the production rate varies from $10^{23}$ to $10^{30}$ molecules/s over seven different velocities $(0.4-1 \mathrm{~km} / \mathrm{s})$ and produces the curves given in Figure 3.2. To avoid interpolation within the tables, a ninth order polynomial is fitted through each curve with an accuracy of $>0.02 \%$, which then provides an analytic expression for each function. The errors on the ratios are treated as upper and lower bounds which are also calculated with the same polynomial equation to give the errors on the production rates. Only the production rate and velocity parameters are varied; the surface temperature parameter, $\mathrm{T}_{s}$, is kept at $200 \mathrm{~K}$ in the temperature profile.

In order to correct the measurements for temperature sensitivity, we created a second lookup table. It is made in a similar way but now $\mathrm{T}_{s}$ is allowed to vary from $50-300 \mathrm{~K}$, while the velocity parameter is held at a constant value of $0.7 \mathrm{~km} / \mathrm{s}$. Variations in $\mathrm{T}_{s}$ would change the line contrast. The production rate ranges from $10^{24}$ to $10^{29}$ molecules/s. Here, for each value of the production rate, the temperature is increased and the ratio between the two absorption lines is calculated again. Figure 3.3 shows these functions with line area ratio versus continuum temperature. Each contour line represents a different production rate with the lowest rate $\left(10^{24}\right.$ molecules/s $)$ at the top of the figure and the highest rate $\left(10^{29}\right.$ molecules/s $)$ at the bottom. We took the measured MIRO data, overplotted them onto these curves, and performed a linear interpolation between the lines to find the water production rate. This was also performed for the line area ratio errors as before to produce upper and lower bounds on the production rate estimates. For each production rate, as the continuum temperature increases, the ratio between the two absorption lines also increases.

The weaker $\mathrm{H}_{2}{ }^{18} \mathrm{O}$ line is formed mainly near the nucleus. The temperature decreases from the surface, so there is a smaller contrast between the $\mathrm{H}_{2}{ }^{18} \mathrm{O}$ line core and the con- 


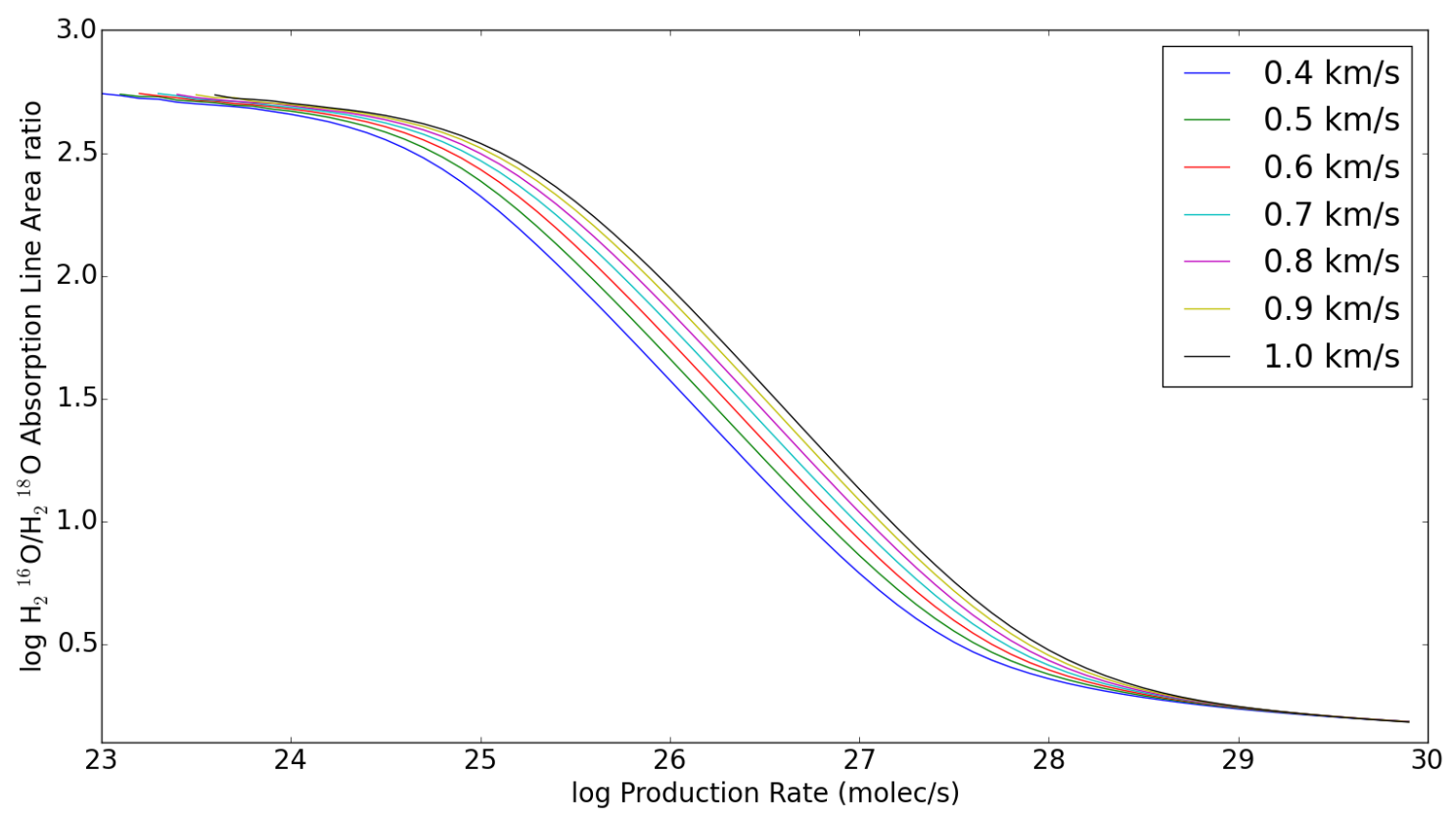

Figure 3.2: Log-log plot of the $\mathrm{H}_{2}{ }^{16} \mathrm{O} / \mathrm{H}_{2}{ }^{18} \mathrm{O}$ absorption line area ratio as a function of production rate for 7 different terminal expansion velocities $(0.4-1 \mathrm{~km} / \mathrm{s})$. For each velocity, as the production rate is increased, the ratio between the two absorption lines decreases from about 500 to approximately 1 . The measured ratio and expansion velocity from the MIRO dataset can be used to derive estimates for the production rate from these curves.

tinuum temperature. On the other hand, the opaque $\mathrm{H}_{2}{ }^{16} \mathrm{O}$ line forms further from the nucleus, at altitudes where the temperature profile is approaching the terminal temperature (estimated to be $30 \mathrm{~K}$, as discussed earlier in this section), and therefore has a larger contrast. Increasing the continuum temperature therefore increases the ratio of line areas between the strong and weak lines.

A second lookup table is needed to test the sensitivity of the production rates to temperature, which was assumed to be constant in the first lookup table. The effect of this second table is a small correction which typically increases the production rates derived from the first lookup table by about 20-25\%. The relative uncertainty on the production rates with these lookup tables are in the range of 30-50\%. This uncertainty grows though with increasing $\mathrm{Q}$ when $\mathrm{Q}>10^{28}$. This particular method is not suitable for deriving water production rates where $\mathrm{Q}>10^{29}$ as the line area versus production rates loses sensitivity above this threshold because both the $\mathrm{H}_{2}{ }^{16} \mathrm{O}$ and $\mathrm{H}_{2}{ }^{18} \mathrm{O}$ lines become saturated. As illustrated in Figures 3.2 and 3.3, the close spacing of the contour lines limits the accuracy on determining $\mathrm{Q}$ from the lookup tables, but as we want to examine the relative behaviour of the production rate from the comet, this method still provides fast and reliable information.

The Haser model gives the global production rate for a spherical comet but our calculated results are local production rates determined for the viewing position of the beam on the comets surface. Each measurement is then a local effective Haser production rate, meaning that the localised beam viewing spot appears to be outgassing as if it was a spher- 


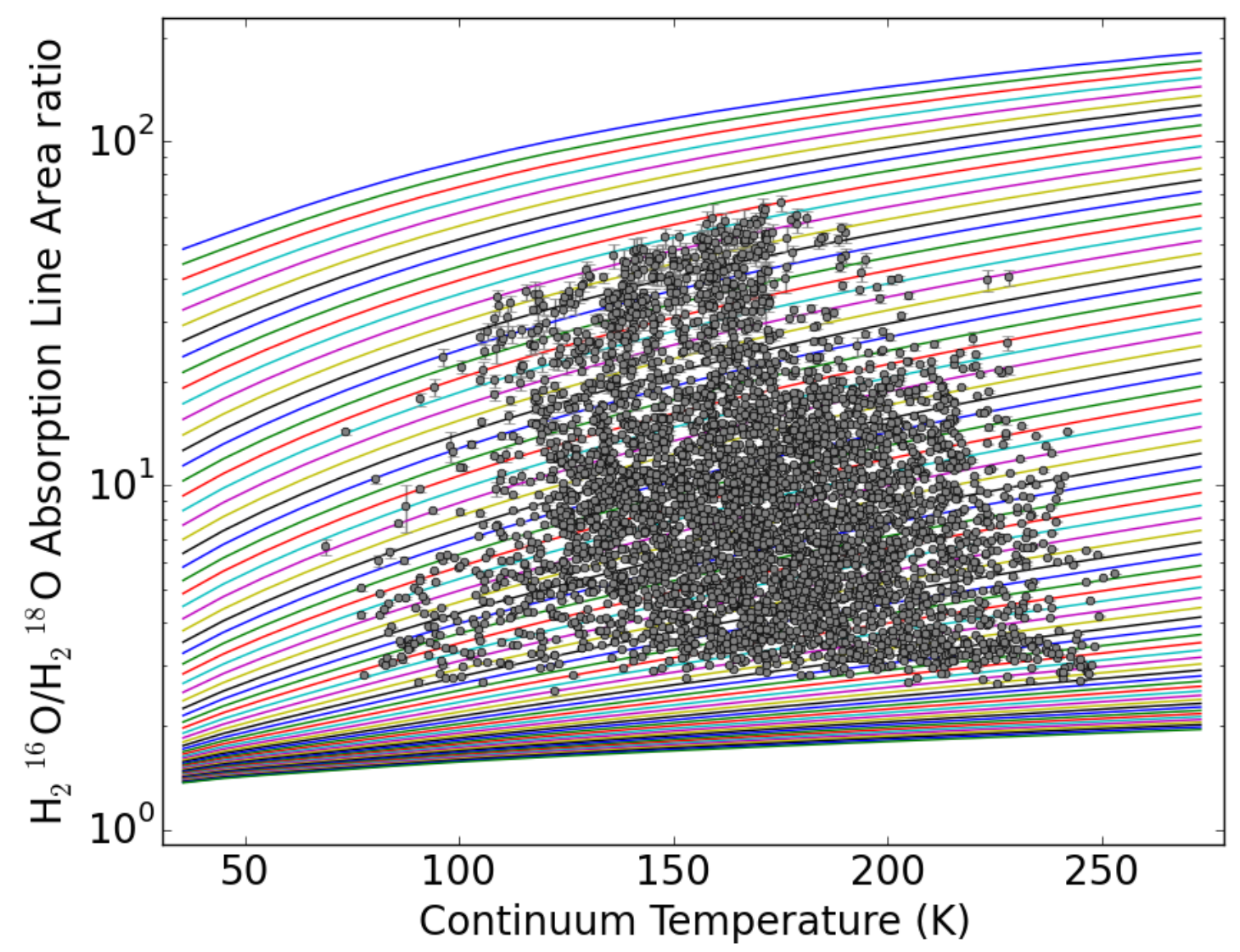

Figure 3.3: Log-linear plot of the $\mathrm{H}_{2}{ }^{16} \mathrm{O} / \mathrm{H}_{2}{ }^{18} \mathrm{O}$ absorption line area ratio as a function of continuum temperature for 101 different production rates $\left(10^{24}-10^{29}\right.$ molecules/s from top to bottom). For each production rate, as the continuum temperature increases, the ratio between the 2 absorption lines also increases. The measured ratio and continuum temperature from the MIRO dataset (grey points) can be used to derive estimates for the production rate from these curves.

ical comet with a single global production rate. This local effective Haser production rate is proportional to the derived MIRO column density.

\subsubsection{Thermal sublimation model}

For later interpretation of results, we now describe a basic thermal model for predicting the water outgassing rate, which relies on the comet A model in Keller et al. (2015). We used the SHAP2 shape model (Sierks et al. 2015), which has fewer facets than SHAP5. We performed the described calculations for each facet of the digital shape model, except that we neglected self-heating mechanisms and shadowing. Keller et al. (2015) compare their A, B, and C models with and without self-illumination and show that selfillumination mainly plays a role when the comet is far away from the Sun ( $>3.5 \mathrm{AU}$ ). In the time period examined here, the difference in water production rate is negligible if self-illumination is ignored. The sublimation rate can be expressed as 


$$
\left(1-A_{v}\right) I=\epsilon \sigma T^{4}+Z(T) L_{i c e},
$$

where $\mathrm{A}_{v}$ is the bolometric Bond albedo, taken as $0.01, \mathrm{I}$ is the solar flux received by a facet, $\epsilon=0.9$ is the emissivity, $\sigma$ is the Stefan-Boltzmann constant, T is the facet temperature, $\mathrm{Z}$ is the facet sublimation rate, and $\mathrm{L}_{\text {ice }}=2.6 \times 10^{6} \mathrm{~J} / \mathrm{kg}$ is the latent heat of sublimation for water ice, which we take to be constant. The sublimation rate, water vapour pressure, and thermal velocity are given by (Equation 5 from Panale and Salvail (1984))

$$
\begin{gathered}
Z(T)=2 P(T) /\left(\pi v_{t h}\right) \\
P(T)=3.56 \times 10^{12} \exp (-6141.667 / T)\left[\mathrm{kgm}^{-1} \mathrm{~s}^{-2}\right] \\
v_{t h}(T)=\sqrt{8 R T / \pi \mu}
\end{gathered}
$$

The surface temperature in this model plateaus at around $200 \mathrm{~K}$, which is the same result found for Model A in Figure 4 of Keller et al. (2015). It is worth noting the nonlinear temperature sensitivity of the water vapour pressure and hence the sublimation rate. Increasing the temperature from $140 \mathrm{~K}$ to $200 \mathrm{~K}$ also increases the vapour pressure from $10^{-7} \mathrm{~Pa}$ to $10^{-1} \mathrm{~Pa}$ so that even small variations within this range of temperatures has profound effects on the sublimation rate,

The production rate is proportional to the sublimation rate:

$$
Q=A Z
$$

where $\mathrm{A}$ is the area of the active surface; we assume all facets to be active. By investigating how and why the sublimation rate changes in this thermal model, we can infer how the production rate changes as well.

The shape model gives the average daily illumination of every facet and Equation 3.3 allows for the calculation of the temperature and sublimation rate for each facet. With this information, the average temperature and sublimation rate of the facets across the surface can hence be determined for a given day with these averages weighted by the area of each facet. Using the same latitude bins described in Section 3.5.2, we also calculate the average sublimation rate for six subset regions. It is possible to go one step further, and divide the grid into 20 longitude bins and hence calculate the sublimation rate of 120 sections across the surface. The model is used from 250 days before perihelion to 250 days afterwards to track the behaviour of the sublimation rate. The sublimation rate at each point in time is plotted to determine the $r_{h}$ exponents before and after perihelion. The dataset from the thermal model is trimmed so that we only calculate the exponents over the period of time for where there are observations.

\subsection{Results}

\subsubsection{Water production rate}

Figure 3.4 shows the derived local effective Haser production rates of water from 250 days before and after perihelion. For comparison, we overplotted other measurements made by 
instruments on board Rosetta (red) and ground-based observations (blue). In addition, modelled production rates from Keller et al. (2015) are shown for completeness. There is a general qualitative agreement between all the data included; the MIRO estimates derived here from the lookup tables are consistent with early results (-400 to -100 days from perihelion) from VIRTIS-H (Bockelée-Morvan et al. 2015), VIRTIS-M (Migliorini et al. 2016, Fink et al. 2016), ROSINA-DFMS (Fougere et al. 2016), and ROSINA-COPS (Bieler et al. 2015) along with previous MIRO estimates (Gulkis et al. 2015). Around 120 days before and after perihelion, the derived MIRO estimates and ground-based observations (Bertaux et al. 2014, Crovisier et al. 2002, Feldman et al. 2004, Hanner et al. 1985, Ootsubo et al. 2012, Schleicher 2006) are in rather good agreement, although these were made for previous perihelion passages of comet 67P. In addition, a power law line shown in Fougere et al. (2016) and a model result for a comet with a perpendicular spin axis and an active area of $2 \%$ from Cowan and A'Hearn (1979) cross through the derived MIRO production rates. Hansen et al. (2016) (not shown in Figure 3.4) have derived the long-term variation in the global water production rate of comet 67P from ROSINA data, but our local production rates are generally lower than their findings, as are the VIRTIS measurements (Bockelée-Morvan et al. 2015). We find that the largest local production rate for a single observation is $(1.42 \pm 0.51) \times 10^{28}$ molecules/s on August 29, 2015, and this is a factor of 3 lower than the maximum outgassing given by Hansen et al. (2016). The difference may be because we derive local Haser production rates whereas their work calculates global production rates. Additionally, the two instruments use different acquisition techniques: ROSINA is an in situ instrument whereas MIRO measures line-of-sight spectra. Hansen et al. (2016) discuss how their result is a factor of two larger than results from VIRTIS, which is also a line-of-sight instrument.

The scatter seen in the local water production rates are indicative of the variation in latitude from the subsolar point of each observation. In Figure 3.4, the peaks in the local effective Haser production rates can be seen to occur when MIRO views latitudes close to the subsolar latitude and conversely, the dips correspond to times when latitudes away from the subsolar latitude are observed.

A 14-day moving average smoothing is applied to the MIRO data to give a qualitative description of the mean behaviour over the observational period, shown in orange and in Table 3.1. The moving average assumes that day- and night side observations are equally weighted. The water outgassing increases from $(3.16 \pm 1.00) \times 10^{26}$ molecules/s in the first averaging bin (i.e. 231 days before perihelion, $\sim 2.4$ AU from the Sun) to $(5.05 \pm 2.46) \times 10^{27}$ molecules/s a week before perihelion. Then 245 days after perihelion (the last averaging bin), the water production rate drops to $(1.93 \pm 0.46) \times 10^{26}$ molecules/s. The bias in the latitudinal sampling means that the moving average is close to, but does not represent, the global production rate: the average may over- or underestimate the true global value depending upon the distribution of the observed latitudes. Nevertheless, the qualitative agreement between the moving average and global production rates from other instruments and ground-based observations is good, and these global measurements also fall within the point cloud of the local effective Haser production rates derived in this work.

The black lines for models A and D represent a comet with an even, homogeneous covering of ice across the surface, whereas models $\mathrm{E}$ and $\mathrm{F}$ represent a comet with a spotted, heterogeneous distribution of ice (Keller et al. 2015). Model A reproduces the 


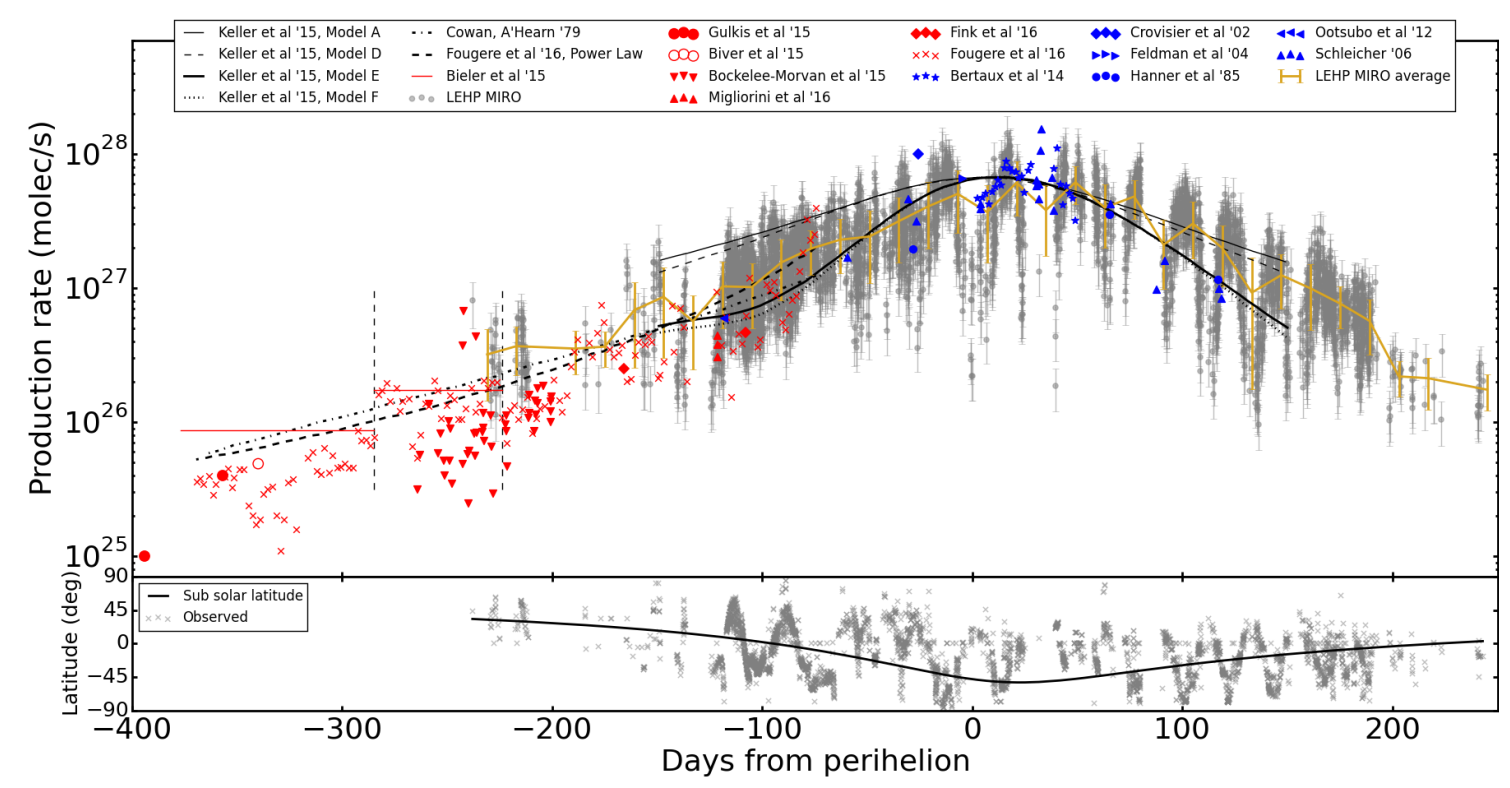

Figure 3.4: Top panel: Calculated local effective Haser production rates of water from 250 days before and after perihelion from the MIRO dataset (grey). Results from other Rosetta instruments of global production rates (red) and ground-based observations from previous perihelion passages (blue) are shown for comparison as well as results from modelling (black lines). A two-week moving average of the MIRO data is also included (orange line). Bottom panel: The latitude for each MIRO observation (grey points) and the position of the subsolar latitude over time (black line).

production rates reasonably well at perihelion but the model values are higher in comparison to other models during the approach to perihelion as also noted in Keller et al. (2015), who found that results were enhanced by a factor of 16 . The case is the same for Model D, which additionally considers thermal inertia. In these two models, all facets are deemed to be active. For models $\mathrm{E}$ and F, which have different values of thermal inertia, some facets are switched off and the abundance of ice is increased elsewhere. The production rate around perihelion for these models does not change in comparison to models $\mathrm{A}$ and $\mathrm{D}$, but the outgassing is reduced pre and post-perihelion. This is more consistent with the derived MIRO results. Hence, qualitatively, the average two week production rate (orange line) appears to be more consistent with the heterogenous surface outgassing as described in models $\mathrm{E}$ and $\mathrm{F}$ in Keller et al. (2015). The authors of this paper also found that these two models were more consistent with pre- and post-perihelion ground-based observations (Schleicher 2006, Hanner et al. 1985, Ootsubo et al. 2012) than the other models.

The data also clearly demonstrate that the maximum production is shifted away from perihelion. To investigate this, linear fits are made through the pre- and post-perihelion data that converge on a solution where the two lines intercept. In order to do this, it is necessary to restrict the dataset, as the $\mathrm{H}_{2}{ }^{18} \mathrm{O}$ line is small at distances greater than about 2.5 AU (before February 2015). As a consequence, the $\mathrm{H}_{2}{ }^{18} \mathrm{O}$ line area can be below the desired signal-to-noise level and without the lowest line areas, we sometimes miss the highest ratios and therefore the lowest water production rate measurements. This can 
be seen in Figure 3.4 in the apparent plateauing of the MIRO results 180 days before perihelion (orange line). For the offset calculation, we hence restrict the dataset to look at results within $1 \mathrm{AU}$ of perihelion (March 2015 - February 2016), where this effect is not prevalent. The intercept from linear fitting in $\log \mathrm{Q}-\log \mathrm{r}_{h}$ space gives the solution as $34 \pm 10$ days after perihelion. Previous work by Bondarenko and Medvedev (2011) and Ferrín (2010) found from cometary light curves that the offset occurred approximately 33 days after perihelion. Schleicher (2006) also found an offset in the peak outgassing from perihelion by 1 month using $\mathrm{OH}$ gas production rates. Furthermore, observations of 67P around its three previous perihelion visits (1996, 2002 and 2009) suggest that the activity peaks $16 \pm 5$ days after perihelion and then again 40 days after perihelion (Bertaux et al. 2014). The offset found here is consistent with Bondarenko and Medvedev (2011), Ferrín (2010) and the second peak from Bertaux et al. (2014), but we are unable to resolve the first peak in this latter work. There is a data gap in mid-September 2015 when the spacecraft goes above $500 \mathrm{~km}$, which limits the determination of the offset. Above $500 \mathrm{~km}$, the MIRO beam is larger than the comet and includes contributions from the background and the nucleus, so MIRO does not collect pure nadir absorption spectra in this period.

\subsubsection{Behaviour with heliocentric distance}

Linear fitting in $\log$ Q- $\log \mathrm{r}_{h}$ space around perihelion of the local effective Haser production rates, allows for the estimation of the change in water outgassing with heliocentric distance by evaluating the $\mathrm{r}_{h}$ exponents, which we define as $\alpha$. We find that these have values of $-3.8 \pm 0.2$ and $-4.3 \pm 0.2$, within $1 \mathrm{AU}$ pre- and post-perihelion, respectively, taking into account the offset described in the last section. This means that the local effective Haser production rate, Q, across the entire surface is roughly symmetrical and proportional to the heliocentric distance, $r_{h}$, as approximately $Q \propto r_{h}^{-4}$. The thermal modelling described in Section 3.4.3 calculates $\alpha$ for pre- and post-perihelion as $-3.5 \pm 0.01$ and $-3.9 \pm 0.03$ for the entire surface.

We further investigated the behaviour by calculating $\alpha$ at different latitudes. We split the dataset into six latitude bins: $90^{\circ}$ to $25^{\circ} ; 25^{\circ}$ to $0^{\circ} ; 0^{\circ}$ to $-20^{\circ} ;-20^{\circ}$ to $-30^{\circ} ;-30^{\circ}$ to $-45^{\circ}$; and $-45^{\circ}$ to $-90^{\circ}$. These bins were chosen so that each bin has the same number of data points (200). As Figure 3.5 shows, $\alpha$ has a latitudinal dependence: the slopes steepen from north to south for pre- and post- perihelion, from $r_{h}$ exponents of about -2 to -6 (right panel). The error bars reflect the standard error of the linear fitting. In addition, the data indicate that there is a line intersection where the post-perihelion slopes are steeper than the pre-perihelion slopes for latitudes greater than $0^{\circ}$, but at more southern latitudes, the slopes are steeper during pre-perihelion than post-perihelion.

To explain this behaviour, we turn to the results of the thermal sublimation model (Keller et al. 2015) which are shown in the left panel of Figure 3.5. This panel shows the $\alpha$ values for the sublimation rate before and after perihelion in the six latitude bins. There is also a steepening of the slopes from north to south similar to the trend seen in calculated production rates. The standard deviation reflects the variation in longitude for each latitude bin.

The steepening of the slopes from north to south in the MIRO data and the model can be explained by the rotation axis of the comet which has been found to be tilted by about 


\begin{tabular}{|c|c|c|}
\hline Date & $\begin{array}{l}\text { Days from } \\
\text { perihelion }\end{array}$ & $\begin{array}{l}\text { Average local production } \\
\text { rate }\left(\times 10^{27} \text { molec } / \mathrm{s}\right)\end{array}$ \\
\hline $25 / 12 / 2014$ & -231 & $0.32 \pm 0.18$ \\
\hline 08/01/2015 & -217 & $0.37 \pm 0.15$ \\
\hline 05/02/2015 & -189 & $0.35 \pm 0.13$ \\
\hline $19 / 02 / 2015$ & -175 & $0.37 \pm 0.11$ \\
\hline 05/03/2015 & -161 & $0.68 \pm 0.43$ \\
\hline $19 / 03 / 2015$ & -147 & $0.86 \pm 0.56$ \\
\hline 02/04/2015 & -133 & $0.57 \pm 0.32$ \\
\hline $16 / 04 / 2015$ & -119 & $1.03 \pm 0.53$ \\
\hline $30 / 04 / 2015$ & -105 & $1.02 \pm 0.51$ \\
\hline $14 / 05 / 2015$ & -91 & $1.55 \pm 0.74$ \\
\hline 28/05/2015 & -77 & $1.97 \pm 0.71$ \\
\hline $11 / 06 / 2015$ & -63 & $2.29 \pm 1.00$ \\
\hline $25 / 06 / 2015$ & -49 & $2.42 \pm 1.34$ \\
\hline 09/07/2015 & -35 & $3.16 \pm 1.60$ \\
\hline $23 / 07 / 2015$ & -21 & $4.07 \pm 2.10$ \\
\hline 06/08/2015 & -7 & $5.05 \pm 2.46$ \\
\hline 20/08/2015 & 7 & $3.68 \pm 2.14$ \\
\hline 03/09/2015 & 21 & $6.11 \pm 2.66$ \\
\hline $17 / 09 / 2015$ & 35 & $3.81 \pm 2.06$ \\
\hline 01/10/2015 & 49 & $6.16 \pm 1.91$ \\
\hline $15 / 10 / 2015$ & 63 & $3.98 \pm 1.99$ \\
\hline 29/10/2015 & 77 & $4.82 \pm 1.52$ \\
\hline $12 / 11 / 2015$ & 91 & $2.10 \pm 1.12$ \\
\hline $26 / 11 / 2015$ & 105 & $3.03 \pm 1.37$ \\
\hline $10 / 12 / 2015$ & 119 & $1.98 \pm 0.96$ \\
\hline $24 / 12 / 2015$ & 133 & $0.93 \pm 0.75$ \\
\hline 07/01/2016 & 147 & $1.25 \pm 0.55$ \\
\hline 21/01/2016 & 161 & $0.99 \pm 0.51$ \\
\hline 04/02/2016 & 175 & $0.77 \pm 0.27$ \\
\hline $18 / 02 / 2016$ & 189 & $0.57 \pm 0.25$ \\
\hline 03/03/2016 & 203 & $0.22 \pm 0.06$ \\
\hline $17 / 03 / 2016$ & 217 & $0.21 \pm 0.09$ \\
\hline $14 / 04 / 2016$ & 245 & $0.17 \pm 0.05$ \\
\hline
\end{tabular}

Table 3.1: Fourteen-day moving average applied to the local effective Haser production rates of water calculated from the MIRO data. 
$50^{\circ}$ to the orbital axis and so the subsolar latitude moves from approximately $40^{\circ} \mathrm{N}$ in August 2014 to $-50^{\circ} \mathrm{S}$ in September 2015, just after perihelion (Keller et al. 2015). The northern latitudes are well illuminated when the comet is further away from the Sun (3.5 AU) but not illuminated during perihelion when the received solar flux increases. As a result, the surface temperature of the northern hemisphere does not change that much in the period for which there are observations and, therefore, neither does the sublimation rate, which is very sensitive to changes in temperature, as already discussed. Conversely, the southern latitudes are not illuminated when the comet is more than 1.8 AU from the Sun, but very well illuminated at perihelion. The change in the surface temperature of the southern hemisphere is quick and as a result, so is the sublimation rate. The resulting exponents for the southern hemisphere are therefore less than in the northern hemisphere.

The line intersection described above is also present in the model, as the post-perihelion slopes are steeper than the pre-perihelion slopes at northern latitudes but the situation is reversed at southern latitudes. In the southern hemisphere, during the pre-perihelion phase, the surface temperature increases very suddenly before perihelion but the decrease in temperature afterwards is slower, so the slope for the sublimation rate is steeper before perihelion than afterwards. For the northern hemisphere, during the approach to perihelion, the sublimation rate changes very little and only increases slightly, whereas the decrease is more pronounced after the approach to perihelion.

The data and model results show that $\alpha$ varies with latitude and that a single $\alpha$ value representing the whole comet surface may not be of fundamental significance. The production rate is a strong non-linear function of temperature, which in turn, depends upon the shape, morphology, and obliquity of the comet, along with the rate of change of illumination; therefore a single $\alpha$ value on its own may not reveal much about comet activity. It appears that the same distribution of ices over a sphere would produce completely different results for $\alpha$. The latitudinal dependence is more important and it can help to reveal areas of the comet that are particularly active or inactive. This can only be investigated by in situ measurements as collected by MIRO.

There are some differences between the $\alpha$ values from the measured production rates and the calculated sublimation rates. However, all of the measurements from the MIRO data are within 1 standard deviation from the model results except for the post-perihelion points north of -20 latitude and the pre-perihelion point north of $25^{\circ}$, which are within 2 standard deviations and have overlapping error bars. For most of points, the standard deviation is between 0.7 and 1.5 but the more southern latitudes have a much larger variation in longitude pre-perihelion. We have already stated that the more southern latitudes experience a sudden increase in temperature but owing to the local morphology of the surface it can be even more extreme at some longitudes than at others. There is then, a broad range of possible $\alpha$ values across the longitudinal plane. Despite the simplicity of the thermal sublimation model that we used here, it captures and reproduces much of the behaviour seen in the MIRO data to a good degree of accuracy. One of our modelling assumptions is that the active area is constant across the comet, which is probably not true (Keller et al. 2015). In addition, the highest $\left(>70^{\circ}\right)$ and lowest latitudes $\left(<-80^{\circ}\right)$ are not really sampled by MIRO and could be excluded from the model; also there may be a longitudinal sampling bias in the data, although this is accounted for in the error bars of the model.

It should be noted that there is a slight difference between how the sublimation rate 


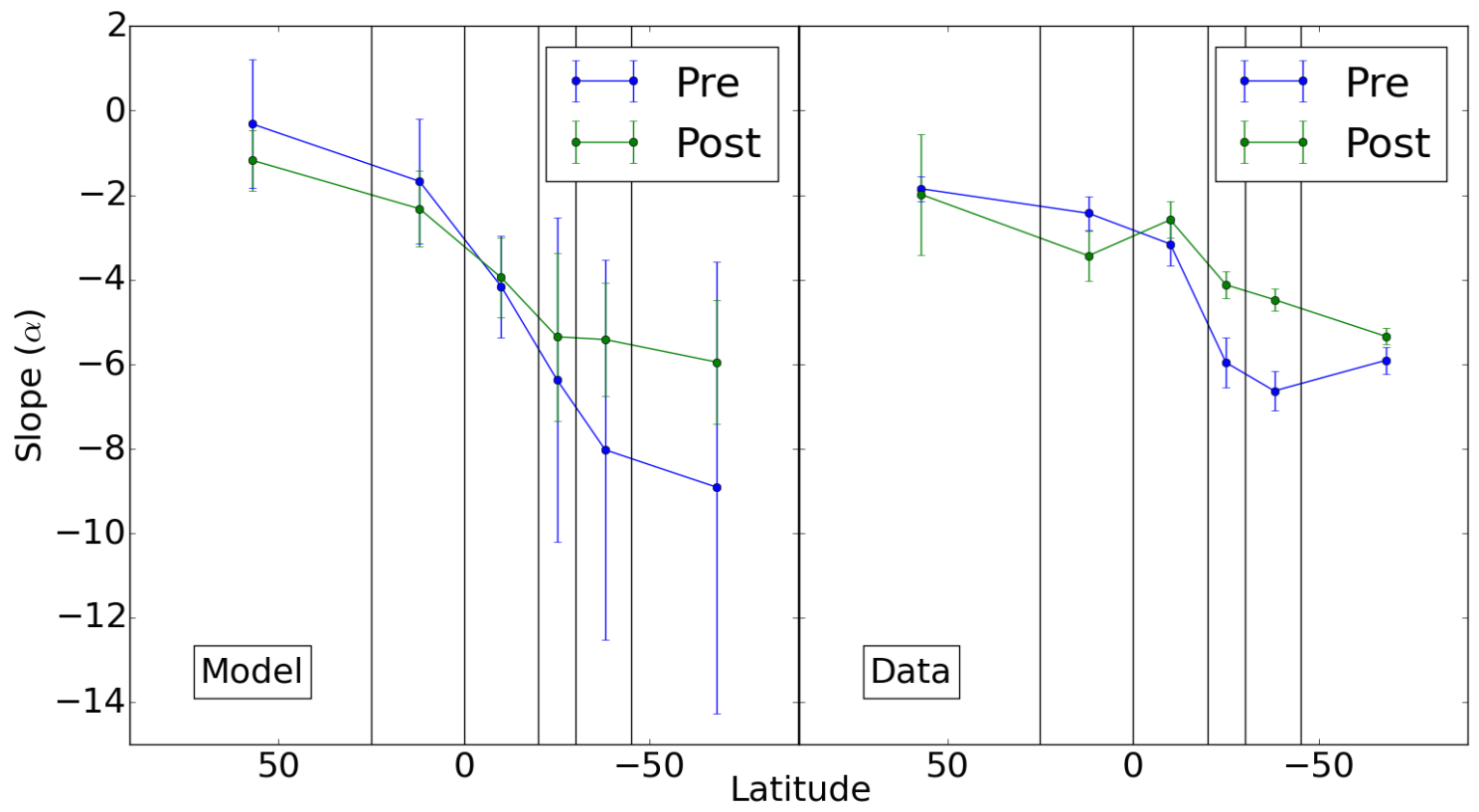

Figure 3.5: (Left) Modelled pre- and post-perihelion slopes $(\alpha)$ of water sublimation rate as a function of latitude averaged over several bins (see text for detailed description). (Right) Pre- and post-perihelion slopes of water production rate from the data derived from MIRO measurements. The error bars on the modelled data reflect longitudinal variability, which is very large in southern hemisphere owing to rapid temperature variations. The MIRO data error bars also reflect the data variability at $1 \sigma$ level.

and the MIRO production rates are calculated. The sublimation rate is calculated from the thermal model, which takes into account a whole latitude band, spanning the dayand night sides, and all longitudes. On the other hand, the production rates only come from a spot on the nucleus where the MIRO beam is observing, which could be day or night, and only at a certain longitude. However, with an appropriate level of sampling (approximately 200 MIRO measurements per latitude bin) enough of the surface is observed to average out the effects of longitudinal variance. A more sophisticated approach where only the facets of the shape model observed by the beam are used to calculate the sublimation rate would be more representative of the actual measurements, but the simple thermal model has captured the essence of the results and can explain some of the features.

\subsubsection{Regional variations}

The large dataset obtained through the fast inversion can be also spatially partitioned to study regional activity variations using the latitude and longitude co-ordinates of the beam position. There are 26 identified regions on 67P defined by El-Maarry et al. (2016) and we can identify the regional source of the produced water from the shape model using the average beam position for each measurement.

First, however, if we assume that molecules move radially away from the comet, then we must restrict our dataset to measurements with a low viewing angle (i.e. the angle 
between surface normal and beam direction) to ensure that we are only observing the production from the region that we are interested in. Using a large viewing angle means that the MIRO beam cuts through layers of the coma where the water production originates from other areas. We hence choose measurements with a viewing angle of less than $30^{\circ}$ to reduce the uncertainty in the regional location of the MIRO beam. This is calculated from the SHAP5 shape model and it is the average viewing angle of all the facets within the beam. We assume that all the contributions to the local effective Haser production rate in the line of sight originate from the MIRO footprint beam. This assumption is only an approximation as it is possible that a particular line-of-sight column density is influenced by other regions owing to the complex nucleus shape and larger phase angles. These potential contributions should be carefully quantified, but it is out of the scope of this work.

The results are given in Figure 3.6, showing regional variations in the water outgassing across the surface of the comet. The average daily solar flux for each region is given in the lower panel for comparison. It is calculated from the solar flux constant and the average cosine solar zenith angle for each facet within the region.

For all regions, the water production rate increases as $67 \mathrm{P}$ approaches perihelion and decreases afterwards. The change in production rate is not uniform though. The southernmost regions (Anhur, Geb, Bes, Sobek, Neith and Wosret) experience a dramatic change in outgassing and are the origins of the largest measured production rates around perihelion. As already mentioned, the subsolar latitude moves south and goes below $-50^{\circ}$ latitude just after perihelion. These southernmost regions are therefore the most illuminated and receive the largest amount of flux at perihelion (between 400 and $600 \mathrm{~W} / \mathrm{m}^{2}$ ). This may result in a stronger depletion of water and potentially other volatiles in these regions.

There are four regions (Atum, Anubis, Khonsu, and Imhotep) at about $-30^{\circ}$ latitude that are also reasonably well illuminated around perihelion (with fluxes between 200 and $400 \mathrm{~W} / \mathrm{m}^{2}$ ) and have reasonable local effective Haser production rates (as high as $\sim 9 \times 10^{27} \mathrm{molec} / \mathrm{s}$ ), although these are slightly lower than most southern regions. As we look further north, to the equator, the water production rates from Ap, Anuket, Maftet, Bastet, Khepry, and Aker are considerably smaller. Due to the obliquity, the northern regions spend most of their time facing away from the Sun at perihelion (whilst the southern hemisphere is well illuminated) so the received flux is lower. This explains why the $\mathrm{H}_{2} \mathrm{O}$ outgassing is lower as we look towards northern latitudes. Indeed, for the regions around the north pole (Seth, Ash, Aten, Babi, and Hapi) as well as on the head lobe (Hathor, Hatmehit, Ma'at, Nut, and Serqet), the production rates are found to be about $50 \%$ lower than in the south at perihelion.

Generally, the regions with high received solar fluxes $\left(>200 \mathrm{~W} / \mathrm{m}^{2}\right)$ have consistently high water production rates (see two leftmost panels in Figure 3.6) and that insolation explains most of the activity. In the other regions, the flux is lower $\left(<200 \mathrm{~W} / \mathrm{m}^{2}\right)$ and the production rates are suppressed. It is notable that, for all regions, the largest production rates are shifted towards perihelion even though in some areas, the received flux is low: for example, in Seth and Ash, the flux drops below $100 \mathrm{~W} / \mathrm{m}^{2}$ but several of the production rate measurements are as high as those seen in the well-illuminated southern regions. Possibly in regions like these, solar insolation is not the only driver of activity but it is hard to draw too many concrete conclusions. It is also possible that these large production 


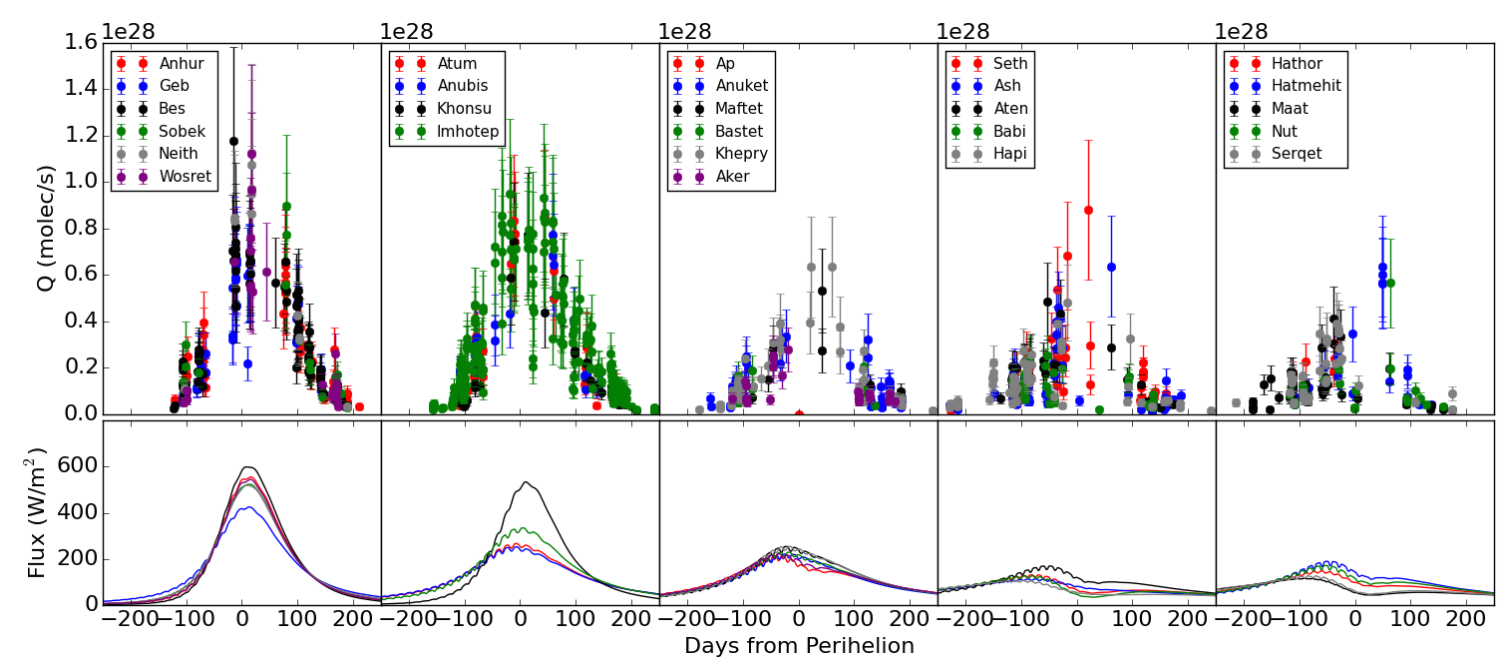

Figure 3.6: Change in water production rate 250 days before and after perihelion sorted by regions, as defined by El-Maarry et al. (2016). The first panel shows the results from the six southernmost regions, Anhur, Geb, Bes, Sobek, Neith, and Wosret. The largest production rates are found here, specifically from the Bes, Wosret, and Neith regions. The second panel contains the results from four regions located around $-30^{\circ}$ latitude: Atum, Anubis, Khonsu, and Imhotep. These show reasonable outgassing rates (as high as $9 \times 10^{27} \mathrm{molec} / \mathrm{s}$ ) and Imhotep in particular is well sampled in this time frame. Six regions that straddle the equator are in the third panel: Ap, Anuket, Maftet, Bastet, Khepry, and Aker. These is an apparent drop here in the production rate around perihelion as we start to look at more northern regions. Seth, Ash, Aten, Babi, and Hapi - the five northernmost regions - are in the fourth panel. Even though these regions do not receive much solar flux due to the rotation axis of the comet, Seth and Ash are still quite active. The remaining five regions, Hathor, Hatmehit, Ma' at, Nut, and Serqet are all located on the head lobe and shown in the fifth panel. The average daily solar flux for each region is shown underneath for comparison.

rates seen in Seth and Ash include contributions in the line of sight of the MIRO beam from other outgassing areas. A more in-depth study of these and other measurements is needed to properly identify any particularly active or inactive areas from the MIRO dataset.

\subsection{Conclusions}

The line area lookup tables method is a fast and computationally inexpensive way to calculate the production rate of water from comet $67 \mathrm{P} /$ Churyumov-Gerasimenko. Our results are consistent with measurements from other Rosetta instruments as well as ground-based observations and produces the time dependence accurately. The uncertainty on each water production rate measurement is between $30 \%-50 \%$. Systematic errors dominate the uncertainty, as can be seen in Figure 3.2, for example, where the ratio curves converge to unity and are flat for large water production rates, so errors in the ratio can give large error bounds on these estimates. In fact, when $\mathrm{Q}>10^{29}$, this method does not provide accurate 
results as the uncertainty can be a factor of 2-3. The errors in the line area measurements from random noise are small, as detailed in Section 3.4.1. The retrieved data variability in a given time period is of the order of $\sigma=2$, which reflects true data variability due to illumination and nucleus inhomogeneities, but also includes modelling errors from simplifications. These however, do not contribute more than $50 \%$. The rapid inversion approach enables us to obtain nearly complete data coverage on the nucleus over time, and study in detail the spatial distribution of outgassing activity and the evolution with heliocentric distance. In combination with the shape model, the dataset can be partitioned by location on the nucleus surface with latitude and longitude co-ordinates. With this, we can determine active regions of the comet and investigate why the activity changes.

The following conclusions can be drawn from the results:

- During the approach to perihelion, the derived results for the local effective Haser production rates of water are consistent with previous global production rates measured by MIRO, ROSINA and VIRTIS.

- The MIRO 14-day mean average changes by an order of magnitude during the 500-day window around perihelion: 231 days beforehand, the outgassing rate is $(3.24 \pm 1.79) \times 10^{26} \mathrm{molec} / \mathrm{s}$, a week before perihelion it is $(5.05 \pm 2.46) \times 10^{27} \mathrm{molec} / \mathrm{s}$, and then 245 days after perihelion it decreases to $(1.72 \pm 0.54) \times 10^{26} \mathrm{molec} / \mathrm{s}$.

- By integrating this average outgassing curve, the comet is found to have lost $(2.4 \pm$ $1.1) \times 10^{9} \mathrm{~kg}$ of water in this time period, which for a dust-to-gas ratio $\gamma=4$ (Rotundi et al. 2015) is $(1.2 \pm 0.6) \times 10^{10} \mathrm{~kg}$ of material or $0.12 \pm 0.06 \%$ of the comets mass, assuming a total mass of $1.0 \times 10^{13} \mathrm{~kg}$ (Sierks et al. 2015). For comparison, Hansen et al. (2016) give the mass loss of water as $6.4 \times 10^{9} \mathrm{~kg}$ from 3.6 AU inbound to 3.0 AU outbound and a mass loss of $4-8 \times 10^{10} \mathrm{~kg}$ in this period. Both of these results are a little higher than what we report here. Additionally, Bertaux (2015) derived a water mass loss of $(2.7 \pm 0.4) \times 10^{9} \mathrm{~kg}$ per orbit from SWAN/SOHO measurements and Keller et al. (2015) calculated the comet would lose $6.5 \times 10^{10} \mathrm{~kg}$ of water during an orbit within their Model A. This latter result is significantly higher than what is derived here for the MIRO results but this is to be expected: Model A in their work assumes a homogeneously active surface, which leads to production rates that are as much as 16 times higher than observations during the early perihelion passage and therefore leads to a higher estimate for the water production over one orbit.

- The maximum local effective Haser production rate of a single observation occurs on August 29, 2015 with a value of $(1.42 \pm 0.51) \times 10^{28} \mathrm{molec} / \mathrm{s}$ or $(426 \pm 153) \mathrm{kg} / \mathrm{s}$. The outgassing appears to peak $34 \pm 10$ days after perihelion. These results are also consistent with ground-based observations of 67P from previous apparitions.

- Linear fitting in $\log \mathrm{Q}-\log \mathrm{r}_{h}$ space finds that the change in the water production rate across the surface of the comet follows power laws of $Q \propto r_{h}^{-3.8 \pm 0.2}$ pre-perihelion and $Q \propto r_{h}^{-4.3 \pm 0.2}$ post-perihelion. A thermal sublimation model derives similar values for the exponents with values of $-3.5 \pm 0.01$ and $-3.9 \pm 0.03$, respectively. The MIRO results also indicate that there is a latitudinal dependence on the production rate for which northern and southern regions have $\mathrm{r}_{h}$ exponents $(\alpha)$, as seen in Figure 3.5. This can be explained by the orientation and orbit of the comet. The 
sublimation rate model driven by the solar illumination indicates that the northern hemisphere does not experience dramatic illumination changes during perihelion approach and it is in shadow 20 days before perihelion. On the other hand, the southern hemisphere suddenly becomes illuminated at perihelion, causing a dramatic increase in the sublimation rate. The subsolar latitude moves south quickly in the first half of 2015 but the return north is slower after perihelion.

- The retrieved local effective Haser production rates can also be used to identify the most active regions on the comet, particularly those that experience an increase in illumination around perihelion. The southernmost regions, including Wosret, Neith, and Bes, are the most active regions in this period. Despite the fact that the received solar flux in the northernmost regions and on the head lobe drops significantly (see rightmost panels in Figure 3.6), the local effective Haser water production rates can still be relatively high; for example, in the Seth region just after perihelion, the production rate was measured to be $\sim 9 \times 10^{27} \mathrm{molec} / \mathrm{s}$ even though the average daily flux was $\sim 50 \mathrm{~W} / \mathrm{m}^{2}$. Further work is needed to quantify the effects of geometry and phase angle on the water column determination from these particular regions.

As noted by Maquet (2015) and Guzzo and Lega (2015), comet 67P has had a chaotic orbital history. It was only brought onto an orbit with a perihelion distance of $1.3 \mathrm{AU}$ after a close encounter with Jupiter in 1959. The comet has now experienced nine perihelion passages at this distance. Before the 1959 encounter with Jupiter, the estimated closest approach to the Sun was 2.7 AU. Even though this comet might have spent most of its time relatively far from the Sun, it is likely that the major observed geomorphological forms, the apparent layering, and the variations in lateral volatile ice are all a result of evolutionary processing (Guilbert-Lepoutre et al. 2016). In addition, the loss of volatiles is currently modulated by the actual nucleus shape and spin axis orientation (Keller et al. 2015). Modern models of the long-term evolution of cometary bodies demonstrate that even a non-uniform surface albedo distribution produces varying thermal gradients, which leads to inhomogeneous ice distribution and composition (Guilbert-Lepoutre and Jewitt 2011). Rosenberg and Prialnik (2010) also show strong evidence that an initial heterogeneous distribution of internal porous ice patches strongly affects the production rates and mass loss. In this connection, it is conceivable that the chaotic path of 67P may have induced the radial and lateral distribution of the cometary refractory and volatile content in the comet, which could explain the observed activity pattern of different volatiles. Huebner et al. (2006) provide a comprehensive overview of the effects of multi-stage injection into the inner solar system on the internal structure of the nucleus. Hence, establishing a possible relationship between the present state and the original state of the comet is an important but difficult and unresolved task, which includes the estimates of the fraction of volatiles that the comet $67 \mathrm{P}$ has lost so far. Appropriate modelling efforts are needed to yield insights into these questions. 



\section{Paper II - On the interpretation of heliocentric water production rates of comets}

This chapter has been accepted for publication by A\&A as the journal article: Marshall, D., et al. "On the interpretation of heliocentric water production rates of comets."

\subsection{Summary}

We investigate the influence of three basic factors on the water production rate as a function of heliocentric distance: nucleus shape, the spin axis orientation, and the distribution of activity on a comet surface. A basic water sublimation model driven by solar insolation is used to derive total production rates for different nuclei shapes and spin axis orientations using the orbital parameters of $67 \mathrm{P} / \mathrm{Churyumov-Gerasimenko.} \mathrm{The} \mathrm{slopes} \mathrm{of}$ production rates versus heliocentric distance are calculated for the different model setups. The standard (homogeneous) outgassing model confirms the well known result regarding the heliocentric dependence of water production rate which remains invariant for different nuclei shapes as long as the rotation axis is perpendicular to the orbital plane. When the rotation axis is not perpendicular, the nucleus shape becomes a critically important factor in determining the water production curves as the illuminated cross-section of the nucleus changes with heliocentric distance. Shape and obliquity can produce changes in the illuminated cross section of up to $50 \%$ over an orbit. In addition, different spin axis orientations for a given shape can dramatically alter the pre- and post-perihelion production curves, as do assumptions about the activity distribution on the surface. If, however, the illuminated cross-section of the nucleus is invariant then the dependence on the above parameters is weak, as demonstrated here with 67P/Churyumov-Gerasimenko shape. The comets Hartley 2 and Wild 2 are shown to yield significantly different production curve shapes for the same orbit/orientation as 67P/CG, varying by as much as a factor of 3 as a result of only changing the nucleus shape. Finally, we show that varying just three basic parameters, shape, spin axis orientation, and active spots distribution on the surface, can lead to arbitrary deviations from the expected inverse square law dependence of water production rates near $1 \mathrm{AU}$. With the obtained results we cannot avoid the conclusion that, without prior knowledge of basic parameters (shape, spin-axis orientation, activity locations), it is difficult to reveal the nature of cometary outgassing from the heliocentric water production rates. Similarly, the inter-comparison of water production curves of two such comets may not be meaningful. 


\subsection{Introduction}

Comets are icy bodies which heat up as they approach the sun and sublimate volatile material from their nuclei to create a surrounding tenuous gas coma. Water vapor is generally the dominant component of the coma, especially around perihelion (BockeléeMorvan, D. et al. 2002, Hässig et al. 2015), however at larger heliocentric distances where it is colder and the water ice is more stable, more volatile species such as CO can dominate the coma, as is the case for 29P/Schwassman-Wachmann (Bockelee-Morvan et al. 2010). Biver et al. (1997) show that for comet Hale-Bopp, the outgassing transitions from $\mathrm{CO}$ to $\mathrm{OH}$ dominated at approximately 3.5 au and Laeuter et al. (2018) show that the water production rate can be lower than the production rates for $\mathrm{CO}$ and $\mathrm{CO}_{2}$ from 67P/Churyumov-Gerasimenko beyond 3.5 au post-perhelion. The water production rate, $Q$ [molec. $\mathrm{s}^{-1}$ ], increases as the distance between the nucleus and the sun decreases due to increasing solar flux, nearly with an inverse square law dependence, but this is heliocentric distance dependent: for volatile ices such as $\mathrm{CO}$ and $\mathrm{CO}_{2}$, the production rates can be theoretically approximated by an inverse square law $\left(r_{h}^{-2}\right)$ at distances less than $\sim 4$ au; for water, which has a higher sublimation temperature, the slope is usually much steeper between 1-4 au, and only approaches $r_{h}^{-2}$ within 1 au of the sun (Cowan and A'Hearn 1979). As noted in Steckloff et al. (2015) and Steckloff and Jacobson (2016), by fully considering the temperature dependence of the sublimation coefficient (Gundlach et al. 2011), the production rate approaches $r_{h}^{-2}$ within 0.5 au.

Cometary water production rate curves are unique for each object and show few common features. Some comets exhibit reasonably symmetric water production rates about perihelion (Hale-Bopp, Kührt (1999)), while others show asymmetric behaviour (67P/Churyumov-Gerasimenko, Hansen et al. (2016)). In addition, the peak production does not necessarily occur at perihelion as it approximately does for $2 \mathrm{P} /$ Encke and 46P/Wirtanen, but it can be offset to appear post-perihelion (as for 30P/Reinmuth 1 and $6 \mathrm{P} / \mathrm{d}$ 'Arrest) or even pre-perihelion (as for $22 \mathrm{P} /$ Kopff and $81 \mathrm{P} /$ Wild 2) (Bondarenko and Medvedev 2011).

The observed slopes for the water production rate also vary between comets. From the Rosetta mission, several attempts have been made to estimate the slopes for the change in the water production rate with heliocentric distance $\left(Q \sim r^{N}\right)$ for 67P/CG (Hansen et al. 2016, Wedlund et al. 2016, Marshall et al. 2017), giving values of $\mathrm{N}=-5.3,-3.8$ and -7.1 pre-perihelion, and $\mathrm{N}=-7.1$ and -4.3 post-perihelion. Values for $\mathrm{N}$ have also been obtained for other comets, including Hale-Bopp ( $\mathrm{N}=-1.88$, Russo et al. (2000)) and 153P/Ikeya-Zhang ( $\mathrm{N}=-3.21$, Russo et al. (2004)). It is worth pointing out that all of these values have been obtained over different ranges of heliocentric distance.

Although suitable from an observational point of view, we raise a question: what is the exact physical relevance of these slopes to the activity and given nucleus? And how can slopes for different comets be compared?

In particular, we explore how the three basic factors - nucleus shape, obliquity of the rotation axis, and activity distribution - affect the water production rate of comets and their respective slopes ${ }^{1}$. It has been known for a long time (Sekanina 1981) that the orientation of the spin axis is very important for the light curves (brightness) of comets,

\footnotetext{
${ }^{1}$ The Python code used in this chapter is available to download from: https://gitlab.com/david_marshall_mps/comet-outgassing-QvRh
} 
with highly oblique objects like $6 \mathrm{P} / \mathrm{d}$ 'Arrest being able to sustain their brightness weeks after perihelion. We also discuss how our simplified model can be improved to add further realism. However, with more free parameters added to the problem it is unlikely that we can break the demonstrated degeneracy, if at least the basic nucleus shape, spin-axis orientation, and the active spot distributions are unknown.

\subsection{Sublimation model}

Following early works such as Watson et al. (1962), Cowan and A'Hearn (1979) and Weissman and Kieffer (1981), as well as Steckloff et al. (2015), the sublimation of water per unit area from an icy body can be written as:

$$
\frac{S_{o}\left(1-A_{v}\right)}{r_{h}^{2}}=\epsilon \sigma T^{4}+Z(T) L_{i c e},
$$

where $\mathrm{S}_{o}$ is the solar flux with a value of $1367 \mathrm{Wm}^{-2}, \mathrm{~A}_{v}$ is the bolometric Bond albedo with a value of 0.01 (Lamy et al. 2007), $r_{h}$ is the heliocentric distance (dimensionless), $\epsilon$ is the emissivity with a value of $0.9, \sigma$ is the Stefan-Boltzmann constant, T is the surface ice temperature, $\mathrm{Z}(\mathrm{T})$ is the sublimation rate $\left[\mathrm{kg} \mathrm{s}^{-1} \mathrm{~m}^{-2}\right]$ and $\mathrm{L}_{\text {ice }}$ is the latent heat of water ice with a value of $2.6 \times 10^{6} \mathrm{~J} / \mathrm{kg}$, taken to be a constant. This is similar to the form given in Keller et al. (2015). The rate of sublimation can be calculated from the equations (Panale and Salvail 1984, Langmuir 1913):

$$
\begin{gathered}
Z(T)=2 P(T) /\left(\pi v_{t h}(T)\right) \\
P(T)=3.56 \times 10^{12} \exp (-6141.667 / T)\left[\mathrm{kgm}^{-1} \mathrm{~s}^{-2}\right] \\
v_{t h}(T)=\sqrt{8 R T / \pi \mu} .
\end{gathered}
$$

The constants in Eq. 4.3 are from solving the Clausius-Clapeyron equations. $v_{t h}$ is the thermal velocity, $R$ is the gas constant and $\mu$ is the molar mass of water.

The water production rate is simply the product of the sublimation rate of a region, $Z_{\text {region }}\left(r_{h}\right)$, and the sublimating area of the region, $A_{\text {region }}$. As we only use digital shape models in this work, the regions we describe are the triangular facets of a shape model. Summing over all facets, where $N$ is the number of facets and $i$ is the ith facet, gives the total water production rate:

$$
Q\left(r_{h}\right)=\Sigma_{i}^{N} Z_{i}\left(r_{h}\right) A_{i} .
$$

For the sake of simplicity and to isolate the effects of spin axis orientation, shape, and the distribution of activity on the surface, Eq. 4.1 is presented only for highly idealized conditions of pure surface ice with zero thermal inertia. For the same reason we also neglect the temperature dependence of the sublimation coefficient (Gundlach et al. 2011). We assume that the cometary objects in this work are only made of water ice. These idealistic simplifications limit the validity of our model to heliocentric distances up to $\sim 3$ $4 \mathrm{au}$ (Meech and Svoren 2004). At larger distances, the heat conductivity term becomes significant and is dominated by the radiative part (Skorov et al. 2017). However, for detailed modeling, the composition, structure, and microphysical properties of the near 
surface layer must be known. We acknowledge that cometary activity is more complex than assumed here (the so called Whipple model (Whipple 1950)), and additional process such as ice phase changes near the sub-surface, extended sources (such as inferred for 103P/Hartley2), and super-volatile release impact water production rates and so does their surface distribution. Nevertheless, all of these remain poorly constrained apart from a few comets visited by spacecrafts (usually over a limited $r_{h}$ range) and would only add unnecessary complexity and additional unconstrained parameters.

With the stated assumptions, when the total flux term (the left side of Eq. 4.1) is low, most of the energy goes into the thermal re-radiation term, and the sublimation term is negligible. As the total flux increases, more energy goes into sublimation and from approximately $50-1000 \mathrm{Wm}^{-2}$ (or $160-200 \mathrm{~K}$ surface temperature), the sublimation and thermal re-radiation terms on the right side are both similar in magnitude. At fluxes greater than $1000 \mathrm{Wm}^{-2}$ (or $200 \mathrm{~K}$ surface temperature), the sublimation flux accounts for more than $90 \%$ of the total flux and dominates over the thermal re-radiation term. When the flux is large $\left(>1000 \mathrm{Wm}^{-2}\right)$, the thermal term does not contribute much, and Eq. 4.1 reduces to:

$$
\frac{S_{o}(1-A)}{r^{2}} \approx \frac{Q}{A} L_{i c e}
$$

replacing $\mathrm{Z}$ with Eq. 4.5. It can be seen that for large fluxes, for instance when a comet is close to the Sun, the water production rate follows an inverse square law for the heliocentric distance.

For the numerical modelling described in this work, we use the sublimation model in Equation 4.1 to calculate the received flux and hence water production rate for a comet on an orbit around the Sun. We use a 67P-like orbit (eccentricity $=0.64$, semimajor axis $=3.46$ ) and change the shape of the object on the orbit. For every facet of an object, the received flux can simply be calculated from the heliocentric distance and the orientation of the facet normal vector and the sun vector. Although important for very precise modeling of activity, shadowing and self-heating are not included in our considerations of realshape illumination conditions. The received flux then corresponds to a temperature and sublimation rate given in a pre-calculated lookup table. The production rate for each facet is found by multiplying the facet sublimation rate by the facet area. The orbit is divided into fifty intervals and at each interval, the temperature, sublimation rate and production rate is determined for every facet over a single comet rotation. The average sublimation and production rates are then calculated for each heliocentric distance by averaging over all facets over one rotation. For simplicity we neglect the effects of selfheating for irregular shapes, which does not invalidate the main points of this work as demonstrated in Keller et al. (2015).

The model does not need to account for a rotation rate. At each heliocentric distance interval, the comet is simply rotated and the water production is calculated for each interval during a comet day.

This enables us to probe the effects of different shapes on the change in production rate with heliocentric distance. We also investigate the effect of the obliquity angle, defined as the angle between the rotation axis and the normal to the orbital plane, with the rotation axis in the same direction as the semimajor axis. In addition, we consider $\Phi$, the argument of the subsolar meridian at perihelion (hereafter, called argument $\Phi$ ) defined as the angle 
swept out by the rotation axis from the semi major axis (Sekanina 1981). Obliquity and argument $\Phi$ are rotations of the spin axis in two different perpendicular planes. We choose this method to demonstrate the effects of these two angles of axis orientation separately. Finally we study the effect of random and non-random distributions of activity on the surface.

\subsection{Results}

\subsubsection{Effect of comet shape and obliquity}

We use six comet shapes (a sphere, 67P (Preusker et al. 2015), 81P/Wild2 (Farnham et al. 2005), 103P/Hartley 2 (Farnham and Thomas 2013a), 1P/Halley (Stooke 2002) and 9P/Tempel 1 (Farnham and Thomas 2013b)) and solve Eq. 4.1 for each comet around a $67 \mathrm{P}$-like orbit. This is done for obliquity values of $0^{\circ}, 30^{\circ}, 45^{\circ}, 60^{\circ}$ and $90^{\circ}$, keeping $\Phi$ constant (equal to 0 ).

We note that these shape models come from a variety of space mission flybys which do not have full coverage and only tens of metre resolution (except for the 67P shape model). However, this is not a problem for us as we only interested in how the shape affects the production rate curve. In addition, the errors and resolution of the shape models are not important for our model, the overall nucleus shape of the nuclei is the most important thing and will determine how the water production rate curve changes.

The change in the normalized cross-section of the illuminated active area for each comet at each obliquity is shown in Fig. 4.1. Each curve is normalized to its maximum value. We define the cross-section of the active illuminated area, A, as a summation over all facets:

$$
A=\Sigma A_{f} \cos \left(\theta_{f}\right)
$$

where $A_{f}$ is the area of a single facet and $\theta_{f}$ is the angle between the facet normal vector and the sun-facet vector. If the angle is greater than $90^{\circ}$, it is set to $90^{\circ}$ so that the flux is zero for non-illuminated facets.

For the spherical comet, the illuminated area does not change with heliocentric distance for any obliquity. In fact, regardless of shape, at $0^{\circ}$ obliquity the cross-section of the active illuminated area is constant for any object with heliocentric distance. Despite 67P's irregular shape, its illuminated area does not change much, dropping by only $5 \%$ for an obliquity of $90^{\circ}$.

When the rotation axis is at an oblique angle, the nucleus shapes of 81P/Wild 2 and 9P/Tempel 1 cause significant changes in the illuminated area during an orbit. Additionally, the larger the obliqueness of the rotation axis is, the larger the change in area. For both comets, the illuminated area decreases on approach to the sun before suddenly increasing again within 1 au from perihelion.

Similarly, for 103P/Hartley 2 and 1P/Halley, the elongated shapes of the nucleus result in changes in the illuminated area, this time increasing on perihelion approach and then rapidly decreasing before closest approach. For these two comets, the (illuminated) area changes by more the $50 \%$ from maximum to minimum.

The change in the illuminated area with heliocentric distance has a profound effect on the production rate curves, as seen in Fig. 4.2. The production rate for a sphere at $0^{\circ}$ 
obliquity is shown in dark blue, and the gradient of the production rate curve is shown in the bottom panels. A comet of any shape at $0^{\circ}$ obliquity exhibits a very similar normalised production rate and slope, and a sphere at any obliquity also gives the same normalised result.

The results for $67 \mathrm{P}$ at a rotation axis angle of $60^{\circ}$ is in light blue. Even though we consider a large oblique angle, the production rate curve and the corresponding slopes do not change much in comparison to the spherical case with no axial tilt. Despite the shape and obliquity of 67P, it exhibits similar behaviour to a sphere, as a results of its coincidental invariance of illuminated cross-section with inclination.

The dark and light orange lines lines in Fig. 4.2 represent the production rate and slopes for $81 \mathrm{P} /$ Wild 2 and 103P/Hartley 2 respectively, at $60^{\circ}$ obliquity. In contrast to $67 \mathrm{P}$, these comets yield notable changes in production rates and gradients when obliquity changes, which is due to their shapes that do result in changes of total illuminated area.

In the example for 103P/Hartley 2, the area starts to increase from large heliocentric distances until it reaches a maximum and then decreases in the final approach to perihelion. As a result, the production rate curve is steeper than the other cases far from the sun but less steep close to the sun, and the slope even becomes positive at perihelion. The increasing flux as the comet approaches the sun should increase the production rate but the decreasing illuminated area means that there is less flux available for sublimation.

For Wild 2, the opposite behavior occurs. The illuminated area starts to decrease from large heliocentric distances until it reaches a minimum and suddenly increases as it approaches perihelion. The sudden increases in area results in steeper production rate slopes around perihelion than for any of the tested objects and obliquity setup. In this case, not only does the flux increase for the comet, but also the area, so the increase in water production is enhanced and the slope is steeper.

It is therefore evident that, before even considering any properties of the comet nucleus, the water production rates and their corresponding slopes are heavily dependent upon the obliquity of the rotation axis and shape.

\subsubsection{Effect of activity distributions}

In the previous section, we considered all the surface of the comet to be active. This is unlikely to apply in reality. For example, Keller et al. (2015) and Marschall et al. (2016) show that only certain local activity can explain the outgassing observations of 67P. In this section, we show how features in the production rate curves and their slopes are affected by random and non-random distributions of active regions on the comet.

We examined the production rate curves for six different distributions of activity on a sphere: $100 \%$ active, $50 \%$ randomly distributed activity, $20 \%$ randomly distributed activity, an active region on the northern hemisphere, an active region on the southern hemisphere, and an active belt at $0^{\circ}$ longitude.

For an obliquity of $0^{\circ}$, all the distributions produced similar production rate curves; only the absolute value of $Q$ is affected. The illuminated area stays constant with heliocentric distance and only the magnitude changes depending on how much of the surface is active.

For an obliquity of $60^{\circ}$ though, there are significant differences between the production rate curves for each kind of distribution. These are shown in Fig. 4.3. In the two 


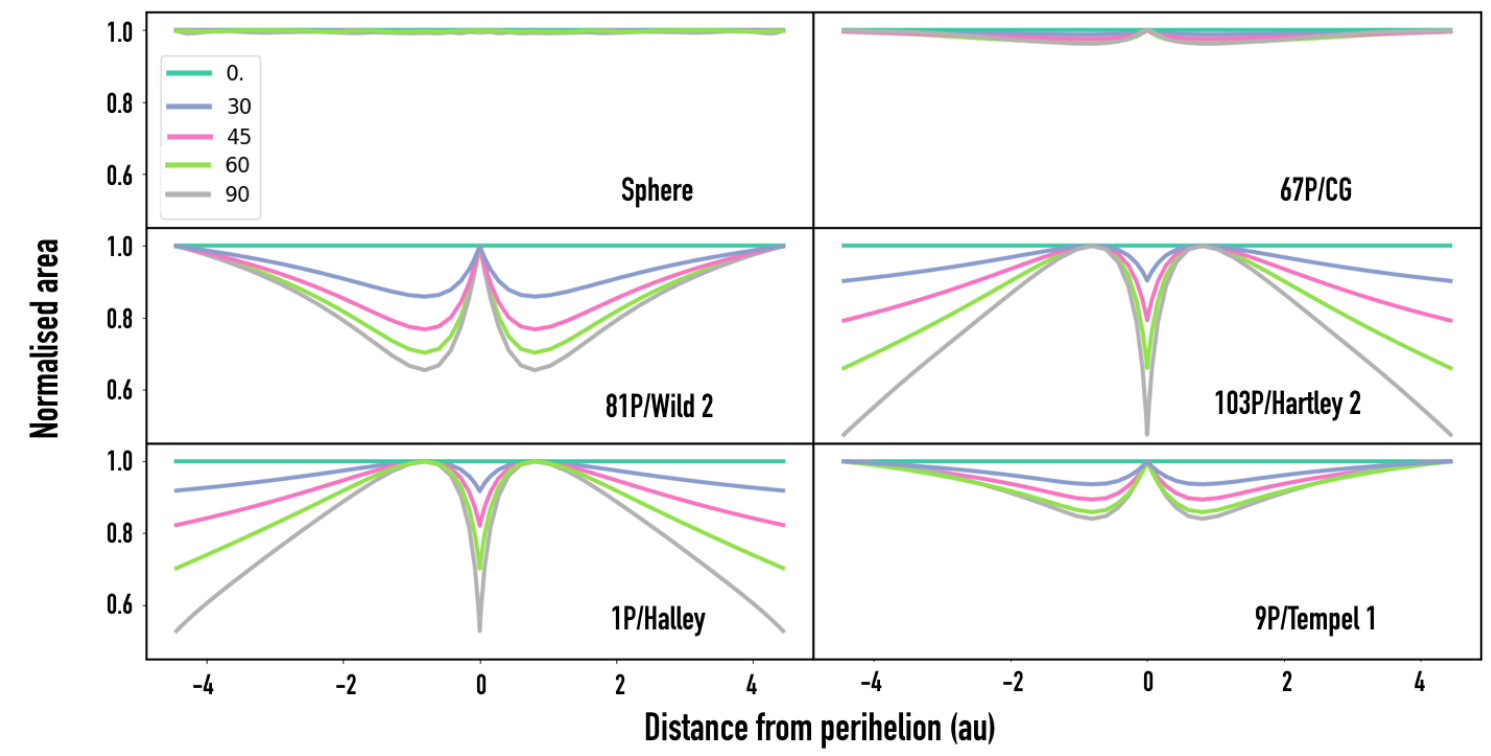

Figure 4.1: The change in the normalised cross-section of the illuminated area for six selected comet shapes (sphere, 67P, 81P, 103P, 1P and 9P) at five different obliquities $\left(0^{\circ}\right.$, $30^{\circ}, 45^{\circ}, 60^{\circ}$ and $90^{\circ}$ ) with distance in au from perihelion (on a 67P-like orbit, perihelion occurs at $1.24 \mathrm{au})$.

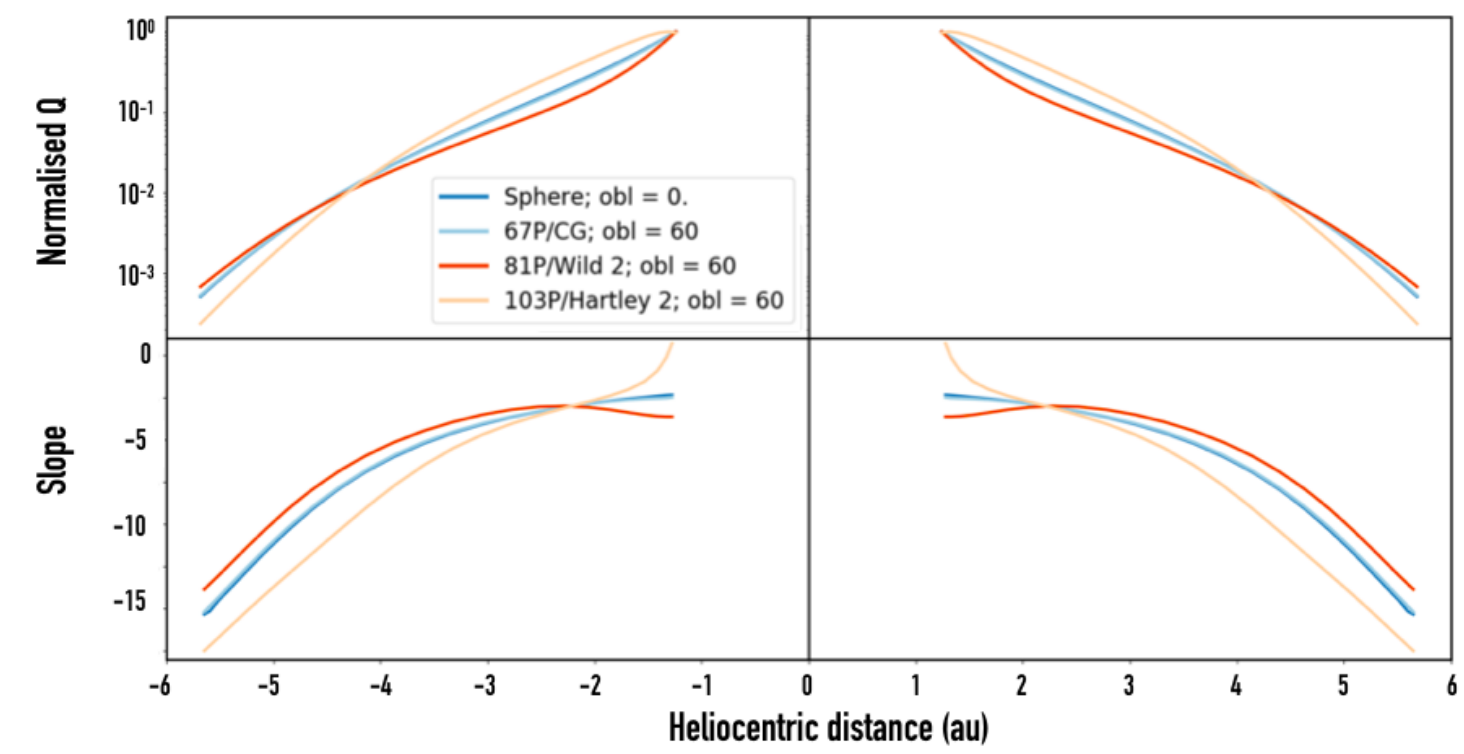

Figure 4.2: Normalised water production rate and slopes of different shaped comets (sphere, 67P, 81P, 103P). A sphere with an obliquity of $0^{\circ}$ is shown as a reference. Any shape at $0^{\circ}$ has a similar production rate curve. Comets 67P, 81P and 103P are shown at obliquities of $60^{\circ}$. The left and right panels correspond to pre- and post-perihelion respectively. Pre-perihelion distances are denoted as negative and post-perihelion distances are given as positive. For a 67P-like orbit, perihelion occurs at 1.24 au (or -1.24 au in the pre-perihelion figures on the left). 


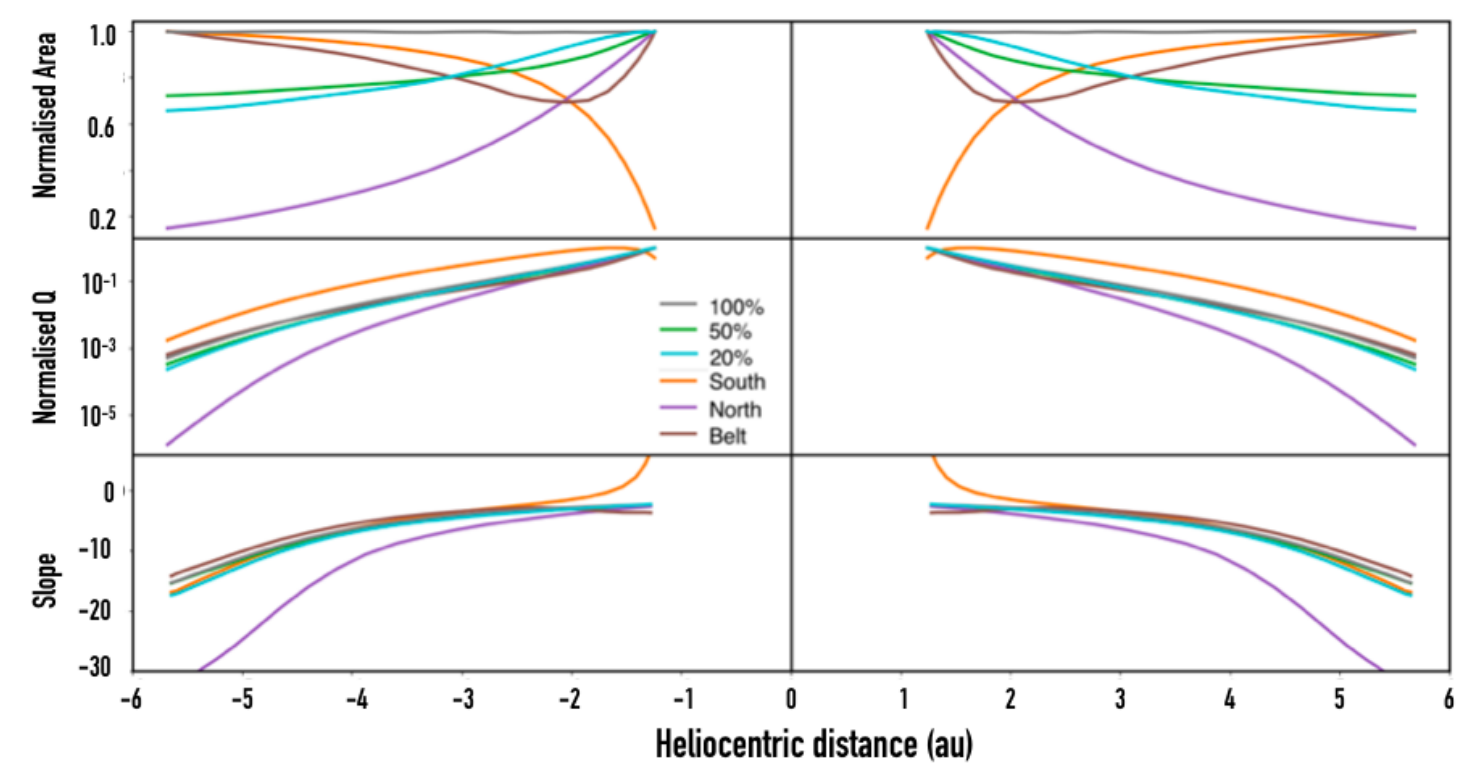

Figure 4.3: Normalised area, water production rate and slopes of spherically shaped comets with different distributions of activity (100\% active, $50 \%$ active, $20 \%$ active, active region on the north hemisphere, active region on the southern hemisphere, active belt at $0^{\circ}$ longitude) at $60^{\circ}$ obliquity.

randomly distributed cases, the illuminated areas are reasonably constant although they can have fluctuations based on how the random activity is distributed. As expected, these fluctuations do not change the production rate curves much and slopes are comparable to the fully active case.

When considering an active region on the northern side, the illuminated area increases dramatically as the comet approaches perihelion, but remains on the night side for long period going to aphelion, due to the assumed obliquity of the rotation axis. The production rate slope starts out very steep, but around perihelion, the curve and slope are comparable to the randomly distributed cases. When the active region is on the southern side, the opposite happens. The active region faces the sun at aphelion and is away from the sun at perihelion. Hence, the illuminated area decreases with heliocentric distance and the slope is therefore less steep at perihelion and becomes positive. The peak in production is offset before and after perihelion.

In the final case, activity is confined to a belt at $0^{\circ}$ longitude. Here, the illuminated area decreases until it reaches a minimum and then increases towards perihelion. This is identical in behaviour to a fully active comet shaped like Wild 2, and both of their production rate curves behave in the same way, with a significant steepening at perihelion. This shows that a sphere with a defined region of activity can have the same production rate curve as an irregularly shaped fully active comet. As a result, it is difficult to disentangle the effects of shape and activity from only the production rate curves. 


\subsubsection{Effects of obliquity and $\Phi$}

In all the simulations so far, the pre- and post-perihelion curves have been symmetrical around perihelion. This is because the obliquity angle has always been orientated towards perihelion (parallel to the semi-major axis), so the change in illuminated area during approach and recession is symmetrical. We now introduce the argument $\Phi$, as defined in Section 4.3. Its effects are actually well known in dealing with light curves of asteroids and comet brightness observations. Here we show that variations in $\Phi$ also have a strong effect on the production rate curves.

We show four selected examples in Fig. 4.4 to illustrate the main point for a 103P/Hartley 2 shaped comet: an obliquity of $0^{\circ}$ and an argument $\Phi$ of $60^{\circ}$; an obliquity of $60^{\circ}$ and an argument $\Phi$ of $90^{\circ}$; an obliquity of $60^{\circ}$ and an argument $\Phi$ of $60^{\circ}$; and an obliquity of $80^{\circ}$ and an argument $\Phi$ of $10^{\circ}$.

For the first case, there is no change in the illuminated area. $\Phi$ changes the angle between the rotation axis and the semimajor axis, but as the obliquity angle is zero, the rotation axis is still perpendicular to the orbital plane. As a result, the illuminated cross section does not change with heliocentric distance and the production curves remain unaltered.

With an obliquity of $60^{\circ}$ and argument $\Phi$ of $90^{\circ}$, the change in illuminated area is the inverse of the change for 103P/Hartley 2 in Section 4.4.1, which had an obliquity of $60^{\circ}$, too. Argument $\Phi$ orients the rotation axis away from the comet-sun vector at perihelion/aphelion, rather than in the same direction, as in Section 4.4.1. Now, the area decreases with heliocentric distance before increasing at perihelion. This has the effect of creating a steep production rate curve around perihelion, similar to the case of Wild 2 in Fig. 4.2. Importantly, differently shaped comets can be seen to have similar behaviours in their production rate slopes for different values of $\Phi$. The pre- and post-perihelion curves are still symmetric around perihelion though.

Asymmetry in the water production can be produced for other values of obliquity and argument $\Phi$. With the obliquity at $60^{\circ}$ and the argument $\Phi$ at $60^{\circ}$, a slight offset can be seen, moving the peak production from 1.24 au (perihelion) to 1.26 au post-perihelion, equivalent to a delay of approximately 16 days. The increase in area post-perihelion is large enough to compensate for the loss of flux from moving out to larger heliocentric distances.

Finally, for case of an obliquity of $80^{\circ}$ and $\Phi$ of $10^{\circ}$, peaks in the production rate can be seen pre- and post-perihelion as the illuminated area reaches a maximum before and afterwards. The peak production can now be seen at 1.4 au pre- and post-perihelion, shifts of about 50 days.

Our modelling is incapable of producing an offset in water production for 67P for an obliquity of $52^{\circ}$ and argument $\Phi$ of $20^{\circ}$, the estimated values for $67 \mathrm{P}$. This is due to the nearly constant behaviour of the illuminated cross section with heliocentric distance. There is not a sufficient increase in area to overcome the decreasing amount of flux received as heliocentric distance increases. A defined active region would have to be used to create an offset in our model. 


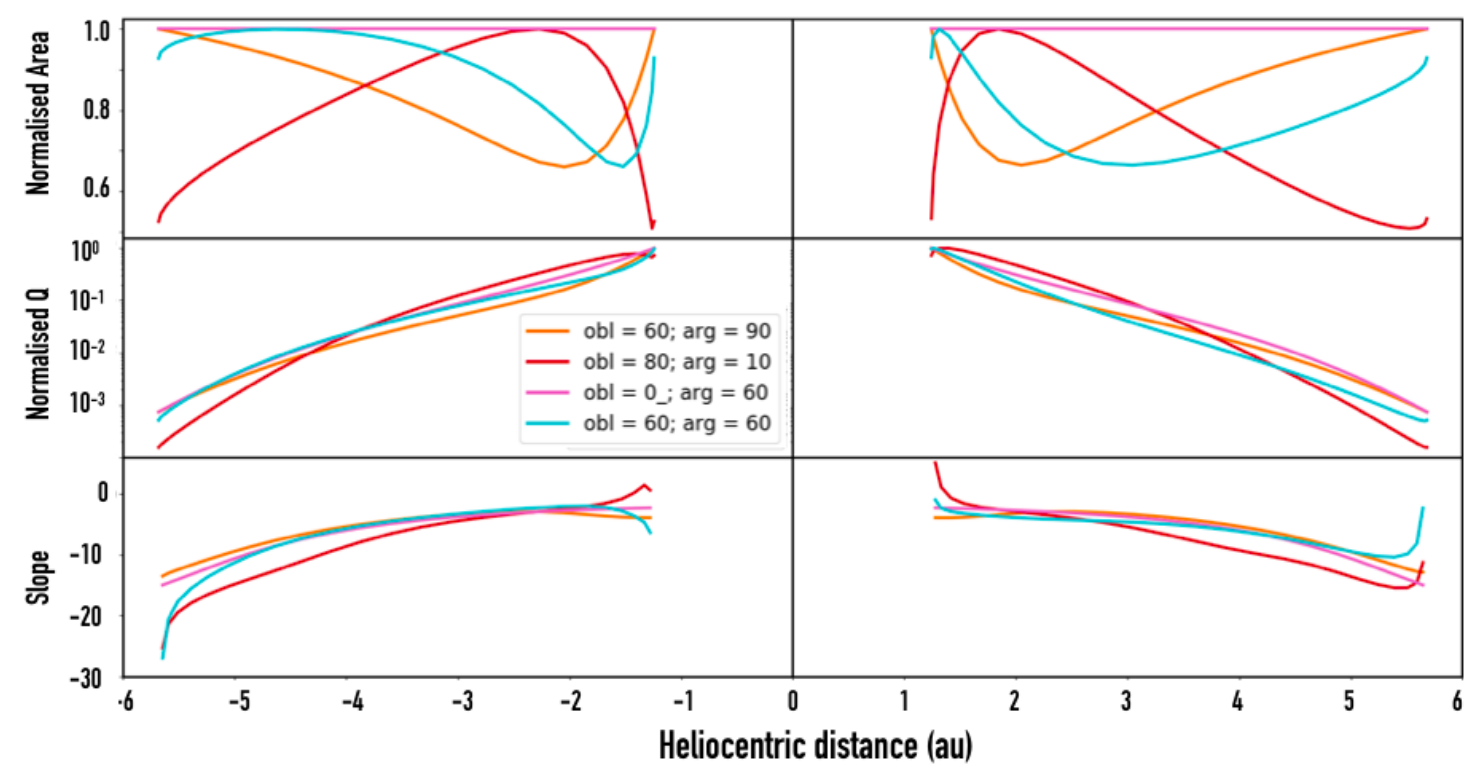

Figure 4.4: Normalised area, water production rate and slopes of 103P/Hartley 2 shaped comets with different obliquities and $\Phi$ angles (obliquity $=0^{\circ}, \Phi=60^{\circ}$; obliquity $=60^{\circ}$, $\Phi=60^{\circ}$; obliquity $=80^{\circ}, \Phi=10^{\circ}$ ). The pre- and post-perihelion curves are plotted on the left and right panels respectively.

\subsection{Conclusions}

We applied a simplified model of water outgassing rate for different cometary bodies to investigate how cometary nucleus shape, spin axis orientation and activity distribution determine the observable dependence with heliocentric distance. We acknowledge that our modeling relies on strong simplifications and does not correspond to any realistic cometary object. Nevertheless, even in this idealistic form it is sufficient to demonstrate the importance of the investigated parameters on the heliocentric production rates of water within 3 au (ie, spin-axis orientation, shape effects, and activity distribution). The application of more comprehensive and detailed models (accounting for self-heating, shadowing, gas diffusion through porous dust mantles with moving boundary, depth variation of conductivity, micro-fracturing, re-condensation effects, perhaps ice-crystallization and number of other effects) is beyond the scope of this work, but they clearly merit future investigations for objects where at least the basic nucleus shape and spin-axis orientation are known. In the introduction we asked: Is there a qualitative and/or quantitative way to interpret the behaviour of $Q\left(r_{h}\right)$ of a comet (for which the exact shape, spin axis and activity distribution are not known), and how to extract meaningful inter-comparisons of such observations for different comets?

The following general conclusions follow from the presented results:

- For homogeneous, solar driven activity, the illuminated cross-section of the nucleus as it travels on its orbit governs the water production rate, $Q\left(r_{h}\right)$, and its gradient with heliocentric distance.

- This variability, even under an assumption of homogeneous icy surface, is signifi- 
cantly modulated by the basic parameters: nucleus shape and spin axis orientation. For example, a comet shaped like 103P/Hartley 2 with a $90^{\circ}$ obliquity produces changes in the illuminated cross-section of $50 \%$ compared to $0^{\circ}$ over an orbit, whereas changes of less than $20 \%$ are seen for a comet shaped like 9P/Tempel 1 at $90^{\circ}$ obliquity.

- The often assumed (approximately) accurate $Q\left(r_{h}\right) \sim r^{-2}$ is valid only for homogeneously active objects at zero obliquity. This relationship holds only over a very narrow heliocentric range around 1 au.

- It is insufficient to derive a single value for the slope in the form, $Q\left(r_{h}\right)=A r_{h}{ }^{B}$, over a large range of heliocentric distances (covering data beyond $3 \mathrm{au}$ ), as the slope, $B$, is also a function of distance. Even in the simplest case of a sphere with 0 degree obliquity, the production rate slope varies from a value of -4 at 3 au to -2.5 at 2 au. Therefore, inter-comparisons of such values among different comets may not be informative.

- If there is a non-uniform distribution of active regions on a comet body (possibly due to any combination of dust cover/mantle, extended sources, albedo, etc), virtually any shape of $Q\left(r_{h}\right)$ can be obtained. This is especially true for irregularly shaped objects with non-zero axis obliquity and $\Phi$ angle.

- The axis orientation (obliquity and $\Phi$ ) is a sufficient condition for irregular shapes to yield asymmetric $Q\left(r_{h}\right)$ and its slopes. The strength of this effect is modulated by the nucleus shape (illuminated cross-section variations).

Inferring physical properties of (unresolved) comets from their production rate curves is an exceptionally difficult task and caution should be exercised when interpreting these curves.

The above conclusions have strong implications on inter-comparisons of the $Q\left(r_{h}\right)$ for different comets, especially when nucleus shape and spin axis orientation are not known. We argue that direct comparison of slopes of heliocentric production rates obtained from different distances as well as mixing pre and post-perihelion data make it very difficult to draw consistent conclusions. It is not rare that such comparisons are published in the literature (usually a result of observational constraints), with one recent example in Wedlund et al. (2016). Such comparisons, as shown in this work, cannot be physically meaningful except by chance.

At last, a suitable example where the shape and spin-axis orientation is well determined but the slope of $Q\left(r_{h}\right)\left[\mathrm{H}_{2} \mathrm{O}\right]$ do not obey the $r_{h}^{-2}$ approximation is the comet 67P. This is surprising, since it retains nearly a constant cross-section despite its spin axis orientation throughout its orbit as shown. From Rosetta measurements the production rate slope ranges from -3 to -8 (Hansen et al. 2016, Marshall et al. 2017), which based on our results can be explained only by an inhomogeneous distribution of water activity. Observations from MIRO and ROSINA have already implied that the activity is not homogeneously distributed, with activity in particular regions being stronger than in other areas (Marshall et al. 2017, Marschall et al. 2016, Fougere et al. 2016). The observed delay in peak production rate for 67P (Hansen et al. 2016, Marshall et al. 2017, Bondarenko and Medvedev 2011) must be a combination of the factors described here, or additional 
processes not considered. Applying more sophisticated models to detailed observations of 67P may provide a better fit, but to what degree such fit is unique is still open. This fact only underlines our general argument using a simple model. 


\section{Paper III - Thermal inertia and roughness of the nucleus of comet 67P/Churyumov-Gerasimenko from MIRO and VIRTIS observations}

This chapter has been published in A\&A as the journal article: Marshall, D., et al. "Thermal inertia and roughness of the nucleus of comet 67P/Churyumov-Gerasimenko from MIRO and VIRTIS observations." Astronomy \& Astrophysics 616 A122 2018. Reproduced with permsission (C) ESO

\subsection{Summary}

Using data from the Rosetta mission to comet 67P/Churyumov-Gerasimenko, we evaluate the physical properties of the surface and subsurface of the nucleus and derive estimates for the thermal inertia (TI) and roughness in several regions on the largest lobe of the nucleus.

We have developed a thermal model to compute the temperature on the surface and in the uppermost subsurface layers of the nucleus. The model takes heat conduction, selfheating, and shadowing effects into account. To reproduce the brightness temperatures measured by the MIRO instrument, the thermal model is coupled to a radiative transfer model to derive the TI. To reproduce the spatially resolved infrared measurements of the VIRTIS instrument, the thermal model is coupled to a radiance model to derive the TI and surface roughness. These methods are applied to Rosetta data from September 2014.

The resulting TI values from both instruments are broadly consistent with each other. From the millimetre channel on MIRO, we determine the TI in the subsurface to be $<80 \mathrm{JK}^{-1} \mathrm{~m}^{-2} \mathrm{~s}^{-0.5}$ for the Seth, Ash, and Aten regions. The submillimetre channel implies similar results but also suggests that higher values could be possible. A low TI is consistent with other MIRO measurements and in situ data from the MUPUS instrument at the final landing site of Philae. The VIRTIS results give a best-fitting value of $80 \mathrm{JK}^{-1} \mathrm{~m}^{-2} \mathrm{~s}^{-0.5}$ and values in the range $40-160 \mathrm{JK}^{-1} \mathrm{~m}^{-2} \mathrm{~s}^{-0.5}$ in the same areas. These observations also allow the subpixel scale surface roughness to be estimated and compared to images from the OSIRIS camera. The VIRTIS data imply that there is significant roughness on the infrared scale below the resolution of the available shape model and that, counter-intuitively, visually smooth terrain (centimetre scale) can be rough at small (micrometre - millmetre) scales, and visually rough terrain can be smooth at small scales. 


\subsection{Introduction}

Comets are considered to be remnants of the pristine material which formed during the early solar system, and as such, they may hold clues to their local formation environment and the evolution of the solar system. One way to assess the pristine nature of a comet is to measure the thermal properties of the surface layer because these control the energy transport through the surface and thus how much material is still unprocessed by solar insolation since its formation.

Energy transport through the surface of the nucleus is a very complex process (e.g. Gortsas et al. 2011). It is mainly initiated by the incoming solar insolation and the dust-ice matrix of the surface allows for the propagation of energy into the subsurface layers. The dust and ice can emit radiation and the volatile ice can also sublimate from the cometary material when heated. Since the volatiles are thought to be distributed throughout the surface and subsurface layers (De Sanctis et al. 2015), sublimation of ices could arise from different depths within the nucleus as heat is transported internally. Dust can then also be expelled from the surface as a result of ice sublimation. In addition, the inhomogeneous surface topography leads to shadowing and self heating (Keller et al. 2015).

Thermal inertia (hereafter TI) and surface roughness are two of the key mediators for the propagation of energy into a comet nucleus. The TI describes how efficiently the solar energy propagates into the surface and represents the ability of the surface to respond to the temperature forcing provided by the solar illumination. Roughness can locally enhance or reduce the amount of energy received and emitted at a given location. These two properties can be determined from the spatial and temporal surface temperature.

The Rosetta mission was launched in 2004 and arrived at its target destination, the comet 67P/Churyumov-Gerasimenko (hereafter 67P), in August 2014. The study of 67P represents an opportunity to better understand the physical properties of the nucleus as inferred from the energy transport in a cometary nucleus. For two years, the spacecraft observed the behaviour of the comet as it approached and receded from the sun. The mission ended in September 2016 when the spacecraft descended onto the surface. There were 11 instruments onboard Rosetta, and we used data obtained by two remote sensing units to determine the thermal properties of the surface and subsurface: MIRO (Gulkis et al. 2007, Microwave Instrument for the Rosetta Orbiter) and VIRTIS (Coradini et al. 2007, Visible and InfraRed Thermal Imaging Spectrometer). These are described in the next section.

Before the Rosetta mission, the Deep Impact spacecraft measured the thermal properties of comets 9P/Tempel 1 and 103P/Hartley 2 (Groussin et al. 2013). The TIs for these two comets were found to be lower than $250 \mathrm{JK}^{-1} \mathrm{~m}^{-2} \mathrm{~s}^{-0.5}$ for Hartley 2 and lower than $45 \mathrm{JK}^{-1} \mathrm{~m}^{-2} \mathrm{~s}^{-0.5}$ for Tempel 1 ( $3 \sigma$ upper limit). The regions with exposed water ice had temperatures over $100 \mathrm{~K}$ higher than the sublimation temperature $(\sim 200 \mathrm{~K})$, indicating the presence of ice-free dust at the subpixel scale. In addition, work by Davidsson et al. (2015) demonstrated that disc-integrated thermal emission from comets depends upon the type of roughness on the surface as well as the TI.

Regarding 67P, in a previous work by Schloerb et al. (2015), the TI in the Imhotep and Ash regions was calculated from MIRO data. These authors derived a low TI between 10$30 \mathrm{JK}^{-1} \mathrm{~m}^{-2} \mathrm{~s}^{-0.5}$ and found that the MIRO millimetre (hereafter $\mathrm{mm}$ ) emission arises from a depth of approximately $4 \mathrm{~cm}$, whilst the submillimetre (hereafter smm) emission comes 
from approximately $1 \mathrm{~cm}$. Their work also shows residuals that are correlated to surface features, suggesting that regional heterogeneity may affect the surface properties of the comet. Choukroun et al. (2015) also calculated TI from MIRO data and obtained values of $10-40 \mathrm{JK}^{-1} \mathrm{~m}^{-2} \mathrm{~s}^{-0.5}$ and $20-60 \mathrm{JK}^{-1} \mathrm{~m}^{-2} \mathrm{~s}^{-0.5}$ for the submillimetre and millimetre channels, respectively. In addition, Gulkis et al. (2015) found low TIs of $10-50 \mathrm{JK}^{-1} \mathrm{~m}^{-2} \mathrm{~s}^{-0.5}$ from MIRO observations as Rosetta first approached 67P.

From the Philae lander, MUPUS (Spohn et al. 2007, Multipurpose Sensors for Surface and Sub-Surface Science) measured a TI of $85 \pm 35 \mathrm{JK}^{-1} \mathrm{~m}^{-2} \mathrm{~s}^{-0.5}$ at the final landing site, Abydos (Spohn et al. 2015). This represents the best-fitting value from their thermal models to the infrared radiometer data from the MUPUS thermal mapper. This is higher than the MIRO measurements but this could be attributed to heterogeneities in the surface layer, such as porosity, temperature, dust-to-ice ratio, composition, dust distribution, and layer thickness (Spohn et al. 2015). The MUPUS measurement is also larger than unresolved observations made by the Spitzer infrared space observatory, which gives a low TI, $20 \mathrm{JK}^{-1} \mathrm{~m}^{-2} \mathrm{~s}^{-0.5}$ (Lamy et al. 2008), and is more consistent with the results obtained from MIRO measurements. The results from thermophysical modelling applied to light curves also gave a low TI value equal to $15 \mathrm{JK}^{-1} \mathrm{~m}^{-2} \mathrm{~s}^{-0.5}$ (Lowry et al. 2012).

For the asteroid Lutetia, which Rosetta passed during its flight to 67P, Keihm et al. (2012) used observations obtained by MIRO and VIRTIS to determine the TI and roughness. These authors found that a low TI $\left(<30 \mathrm{JK}^{-1} \mathrm{~m}^{-2} \mathrm{~s}^{-0.5}\right)$, which increased with depth, and a $50 \%$ fractional surface coverage with hemispherical craters was required to fit the model to the observations.

We aim to determine thermal properties of the surface layer of the nucleus of comet $67 \mathrm{P}$, such as the TI and roughness, by analysing data from the VIRTIS and MIRO experiments. Both of these datasets provide measurements of the emitted and reflected radiance of the cometary nucleus. In the case of VIRTIS, the emitted radiance is related to the surface temperature (uppermost few tens of microns), whereas for MIRO, which operates at longer wavelengths, the emitted radiance is related to the subsurface temperature profile. We focussed our analyses on data obtained in September 2014. This choice was motivated by the opportunity to compare our final results with previous publications from the MIRO science team, such as by Schloerb et al. (2015), Gulkis et al. (2015), and Choukroun et al. (2015), who analysed MIRO data obtained during the same time period although from different regions.

In the next section, we describe the MIRO and VIRTIS instruments. Then, the first step towards estimating the TI involves assessing the datasets to find suitable data for comparison (Section 5.4.1). The thermal model applied to the data from both instruments and used to calculate the temperature gradients is described in Section 5.4.2, and the details of the MIRO radiative transfer model are given in Section 5.4.3. The VIRTIS modelling is described in Section 5.4.4. The results from MIRO and VIRTIS are given in Sections 5.5.1 and 5.5.2, respectively.

\subsection{Instruments}

The MIRO instrument was a microwave spectrometer consisting of a $30 \mathrm{~cm}$ offset parabolic reflector telescope; a millimetre heterodyne receiver, operating at a centre-band frequency 
of $188 \mathrm{GHz}$ (1.6 mm wavelength); and a submillimetre heterodyne receiver, operating at $562 \mathrm{GHz}(0.5 \mathrm{~mm}$ wavelength) (Gulkis et al. 2007). The frequency bands of the millimetre and submillimetre receivers had angular resolutions of $23.8 \mathrm{arcmin}(6.9 \mathrm{mrad})$ and 7.5 arcmin $(2.2 \mathrm{mrad})$, respectively, and each contained a single broadband channel for the measurement of near-surface temperatures. Additionally, a Chirp Transform Spectrometer (Hartogh and Hartmann 1990, CTS) was connected to the submillimetre receiver for the observation of absorption and emission lines of water, carbon monoxide, methanol, and ammonia. The work presented here focusses on the temperatures measured by the two broadband channels.

The instrument observed the source for 30 seconds and was calibrated in situ every 34 minutes by observing onboard hot and cold calibration targets. In September 2014, these typically had temperatures of $18^{\circ} \mathrm{C}$ and $-47^{\circ} \mathrm{C}$. The output from the receivers was proportional to the observed power and converted to an antenna temperature, $T_{A}$, by

$$
T_{A}=P / k,
$$

where $P$ is the observed power density and $k$ is the Boltzmann constant. In this work, we converted the measured antenna temperatures from MIRO to a brightness temperature, $T_{B}$, which is defined as the required temperature of a black body, which fills the antenna beam pattern to produce the observed power. A Planck function was used to calculate $T_{B}$. We assumed that each beam could be approximated as a Gaussian beam, and a multiplicative correction factor of $\frac{1}{0.94}$ was applied to the brightness temperatures from the submillimetre channel as a result of the difference in beam efficiency between the two channels, as described by Schloerb et al. (2015).

The VIRTIS instrument was a hyperspectral imager that consisted of a high-spectralresolution point spectrometer (VIRTIS-H, 1.88-5.0 $\mu \mathrm{m}$ ) and two mapping channels (VIRTIS-M-VIS, 0.22-1.05 $\mu \mathrm{m}$; VIRTIS-M-IR, 0.95-5.1 $\mu \mathrm{m}$ ) (Coradini et al. 2007). We used data acquired by the IR mapping channel VIRTIS-M-IR (hereafter abbreviated as VIRTIS), the cryocooler of which failed in May 2015, thereby ending its science output. Its instantaneous field of view was $250 \mu \mathrm{rad} \times 250 \mu \mathrm{rad}$. At each basic acquisition, VIRTIS recorded a frame, a spectrally resolved spatial line (432 spectral bands times 256 spatial samples; $9.5 \mathrm{~nm}$ spectral sampling). A series of consecutive frames forms a data cube, a spectrally resolved two-dimensional image. For the cubes considered here, a basic acquisition typically had an exposure duration in the order of seconds, whereas the acquisition of a cube takes of the order of tens of minutes up to hours, a time span over which the observation and illumination geometry can change considerably from comet rotation and spacecraft motion.

The VIRTIS infrared spectrometer was mostly sensitive to the surface temperature (uppermost few tens of $\mu \mathrm{m}$ ), rather than the centimetre subsurface as in the case of MIRO. The longest wavelengths that VIRTIS could measure generally provide the best diagnostics since the contribution from reflected flux is less important at these wavelengths. We always see the rising wing on the short wavelength side (the Wien's part) of the thermal emission peak, starting from about 3.0-3.6 $\mu \mathrm{m}$ (Filacchione et al. 2016). 


\subsection{Methods}

\subsubsection{Observational overlap}

In order to better determine the thermal properties of the nucleus in some regions, we decided to combine MIRO and VIRTIS data, providing more observational constraints than with a single instrument and enabling us to investigate possible heterogeneities between surface and subsurface thermal properties. We therefore identified when MIRO and VIRTIS were observing the same location on the comet at the same time. The data were averaged into one-minute time intervals and using a digital shape model of the nucleus, we could determine the footprints on the nucleus of the two instruments at a given time. We used a decimated version of shape model "cg-dlr_spg-shap7-v1.0", which has 124938 facets (Preusker et al. 2017) with a horizontal resolution of approximately $20 \mathrm{~m}$, and the SPICE software (Acton 1996). This way, we could determine the averaged viewing geometry of each observation from the positions of the spacecraft, the comet, and the sun, and then calculate the viewing angle (angle between facet normal vector and the vector connecting the facet and the spacecraft) and beam size of the submillimetre and millimetre beams, as well as the footprint of the observation and the facet illumination. Most of the time MIRO and VIRTIS observed different regions of the nucleus but occasionally they both looked at the same location.

The two instruments acquired data in slightly different ways. The MIRO millimetre and submillimetre beams probed a single circular area (assuming a Gaussian beam shape), giving two subsurface temperatures, one from each beam. At $30 \mathrm{~km}$ from the nucleus the millimetre and submillimetre beams had approximate diameters of $210 \mathrm{~m}$ and $64 \mathrm{~m}$, respectively. The millimetre beam hence observed between 55 and 367 facets of the decimated shape model for the observations given in Table 5.1, and between 3 and 26 facets in the submillimetre beam. The VIRTIS instrument, on the other hand, was a slit spectrometer, observing a narrow swath across the surface at a single exposure. At $30 \mathrm{~km}$, it instantaneously observed an area of approximately $8 \mathrm{~m} \times 2000 \mathrm{~m}$ and could resolve the temperature of the 256 facets placed along the swath. We therefore looked for observations in which the MIRO beam intersects the VIRTIS band. Since both instruments operated at different wavelengths and spatial resolutions, the roughness is treated differently in each case. The MIRO instrument takes large-scale topographical roughness at the scale of the shape model (metres) into account when inferring the TI, and VIRTIS models subfacet roughness, since it has a higher spatial resolution.

In September 2014, observations from the MIRO and VIRTIS instruments overlapped spatially and temporally $\sim 150$ times, and most of these occurred between September 1-2 and September 12-15. For this work, we chose to focus on five of the best times; these times were selected because they all have well-constrained MIRO and VIRTIS observations, good viewing geometries, and cover different areas of the nucleus. These times (specified in UT) are given in Table 5.1 along with the corresponding observed MIRO brightness temperatures. These observations provide data on five areas located on different geomorphological areas (El-Maarry et al. 2015). On September 2 and 15, 2014, the instrument probed areas located in the Aten region, while on September 1 and 13, it observed areas located in the Seth and Ash regions, respectively. On September 12, MIRO and VIRTIS probed an area located at the border between the Ash and Aten regions. The 
5 Paper III - Thermal inertia and roughness

\begin{tabular}{cccccccc}
\hline Date & Time & $\begin{array}{c}\mathrm{smm} \\
\mathrm{T}_{B} \\
(\mathrm{~K})\end{array}$ & $\begin{array}{c}\mathrm{mm} \\
\mathrm{T}_{B} \\
(\mathrm{~K})\end{array}$ & $\begin{array}{c}\text { Local solar } \\
\text { time } \\
\text { (hours) }\end{array}$ & $\begin{array}{c}\mathrm{S} / \mathrm{C} \\
\text { distance } \\
(\mathrm{km})\end{array}$ & $\begin{array}{c}\text { Beam } \\
\text { latitude } \\
\text { (degrees) }\end{array}$ & $\begin{array}{c}\text { Beam } \\
\text { longitude } \\
\text { (degrees) }\end{array}$ \\
\hline Sep 1, 2014 & $23: 48$ & 188 & 173 & 9.9 & 52 & 20 & -143 \\
Sep 2, 2014 & $04: 23$ & 170 & 149 & 10.4 & 53 & 10 & 91 \\
Sep 12, 2014 & $23: 28$ & 157 & 156 & 8.9 & 30 & 45 & 111 \\
Sep 13, 2014 & $09: 33$ & 165 & 163 & 8.5 & 30 & 37 & 174 \\
Sep 15, 2014 & $02: 51$ & 171 & 145 & 10.6 & 30 & 7 & 86 \\
\hline
\end{tabular}

Table 5.1: Brightness temperatures, $\mathrm{T}_{B}$, from the submillimetre and millimetre channels for each MIRO observation, along with the local solar hour, spacecraft distance, latitude, and longitude at the centre of the beam footprint. With an assumed beam efficiency of 0.94 , we use errors on the brightness temperatures of $4 \mathrm{~K}$.

measurements correspond to the pre-landing mapping phase shortly after arrival at the comet. During this epoch Rosetta was orbiting 67P at a distance of $30 \mathrm{~km}$, at $3.38 \mathrm{AU}$ from the sun.

\subsubsection{Thermal model}

To interpret the MIRO and VIRTIS data, we need to calculate the temperature of the surface and subsurface in response to insolation. The thermophysical temperature profile of a comet nucleus can be determined by solving the heat conduction equation with the assumption that the heat induced by the solar radiation is transported into depth and emitted into space (Groussin et al. 2013). This can also be used to sublimate volatiles as considered by Capria et al. (2017), but we neglected this part in this work, since water sublimation is negligible at $3.38 \mathrm{AU}$. The subsurface temperatures can be approximated with a one-dimensional thermal model using the heat equation

$$
\rho c_{p} \frac{\partial T(z, t)}{\partial t}=\kappa \frac{\partial^{2} T(z, t)}{\partial z^{2}},
$$

where $\rho$ is the bulk density in units of $\left[\mathrm{kg} \mathrm{m}^{-3}\right], c_{p}$ is the specific heat $\left[\mathrm{J} \mathrm{kg}^{-1} \mathrm{~K}^{-1}\right]$, and $\kappa$ is thermal conductivity $\left[\mathrm{W} \mathrm{m}^{-1} \mathrm{~K}^{-1}\right]$. The partial derivatives $\frac{\partial T(z, t)}{\partial t}$ and $\frac{\partial T(z, t)}{\partial z}$ are for the changes of temperature with time, $t$, and depth, $z$, respectively. In Eq. (5.2), constant surface properties are assumed that do not depend on depth or temperature or time, and there is no internal heat source. The TI is defined as

$$
I=\sqrt{\kappa \rho c_{p}},
$$

where $I$ has SI units of $\left[\mathrm{J} \mathrm{K}^{-1} \mathrm{~m}^{-2} \mathrm{~s}^{-0.5}\right]$. This is the quantity of interest, and with these equations, the temperature is found as only a function of TI, assuming Bond albedo and emissivity are known. We take the density to be $532 \mathrm{~kg} \mathrm{~m}^{-3}$ (Jorda et al. 2016) and assume the heat capacity to be $500 \mathrm{~J} \mathrm{~kg}^{-1} \mathrm{~K}^{-1}$, estimated from Robie et al. (1970).

For the surface boundary, the following condition is used to solve the heat equation

$$
\frac{S\left(1-A_{h}\right)}{r_{h}^{2}} \cos i=\epsilon \sigma T^{4}-\left.k \frac{\partial T}{\partial z}\right|_{z=0},
$$


where $S$, the solar flux at $1 \mathrm{AU}$, is equal to $1370 \mathrm{~W} \mathrm{~m}^{-2}, A_{H}$ is the Bond albedo, taken to be 0.0108 , from a standard geometric value of 0.04 and phase integral value of 0.27 (Lamy et al. 2007), $\epsilon$ is the infrared emissivity, taken to be 0.95 (Birkebak 1972), $\sigma$ is the Stefan- Boltzmann constant, $r_{h}$ is the heliocentric distance, and $i$ is the angle of incidence of the sun to the local surface.

As a first step, the illumination geometry and projected shadows are calculated for each facet of the shape model over one complete nucleus rotation. This is carried out for three dates for which we have overlapping VIRTIS and MIRO data: September 2, 2014, September 13, 2014, and September 15, 2014.

In the second step, we calculate the temperature of each facet with a thermal model that takes into account insolation, emission, self-heating, and heat conductivity with different values of TI (see Eqs. (5.2 - 5.4) and Groussin et al. (2013)): 5, 10, 20, 40, 80, 160 and $320 \mathrm{JK}^{-1} \mathrm{~m}^{-2} \mathrm{~s}^{-0.5}$. In this work, we test the TI at these discrete values. In the conclusions, rather than discuss error bars, we consider a range of acceptable values denoted by the tested values. The thermal model is run over several nucleus rotations for each date (between 8 and 22 rotations depending on the thermal inertia) with a time step of 12.4 sec and up to a depth of 10 diurnal skin depths (between $2.2 \mathrm{~cm}$ to $1.4 \mathrm{~m}$ depending on the TI). Running to these depths ensures convergence of the temperature profiles up to ten wavelengths of the MIRO millimetre channel $(1.6 \mathrm{~cm})$, and by checking this convergence, we negated the need for a full seasonal model for the uppermost layers of interest. The temperature at the lowest depth was assumed to be $30 \mathrm{~K}$, and a depth is used such that $\frac{d T}{d t}=0$. To account for the large number of facets (124938 facets) and the physical processes such as heat conductivity and self-heating, the code has been optimized and parallelized. On a computer with four CPU cores, it takes approximately 48 hours to run a complete set of TI values at a given date.

As an output, we obtain, for a given date, the temperature profile for each facet of the shape model from the surface to a depth of 10 MIRO mm wavelengths or more. Figure 5.1 shows an example of temperature as a function of depth, for a TI of $80 \mathrm{JK}^{-1} \mathrm{~m}^{-2} \mathrm{~s}^{-0.5}$, on September 15, 2014 (UT 00:00). As expected for the dayside, the temperature decreases with depth. Figure 5.2 shows an example of surface temperature, for varying values of the $\mathrm{TI}$ in the range 5-320 $\mathrm{JK}^{-1} \mathrm{~m}^{-2} \mathrm{~s}^{-0.5}$, on September 15, 2014 (UT 00:00). The maximum temperature decreases with increasing TI (since more energy penetrates into the nucleus by heat conductivity), as does the diurnal thermal amplitude.

\subsubsection{Radiative transfer model for MIRO data}

The MIRO radiometer measures antenna temperatures at millimetre and submillimetre wavelengths, described in Section 5.3, from which brightness temperatures for the observed areas are derived. Using the thermal outputs from Section 5.4.2 as inputs to a radiative transfer model, simulated brightness temperatures (hereafter SBT) are compared to measured brightness temperatures (hereafter MBT) for the different MIRO obervations. The TI of the material layer is constrained if the SBT curve for a given TI intersects the MBT curve. The SBT varies as a function of the TI and the relative complex permittivity (hereafter permittivity) of the material taken into account in the radiative transfer.

The permittivity, $\epsilon$, consists of a real part, $\epsilon^{\prime}$, and an imaginary part, $\epsilon^{\prime \prime}$, which describe the polarisability of a material and the capacity of motion of free charges in the material 

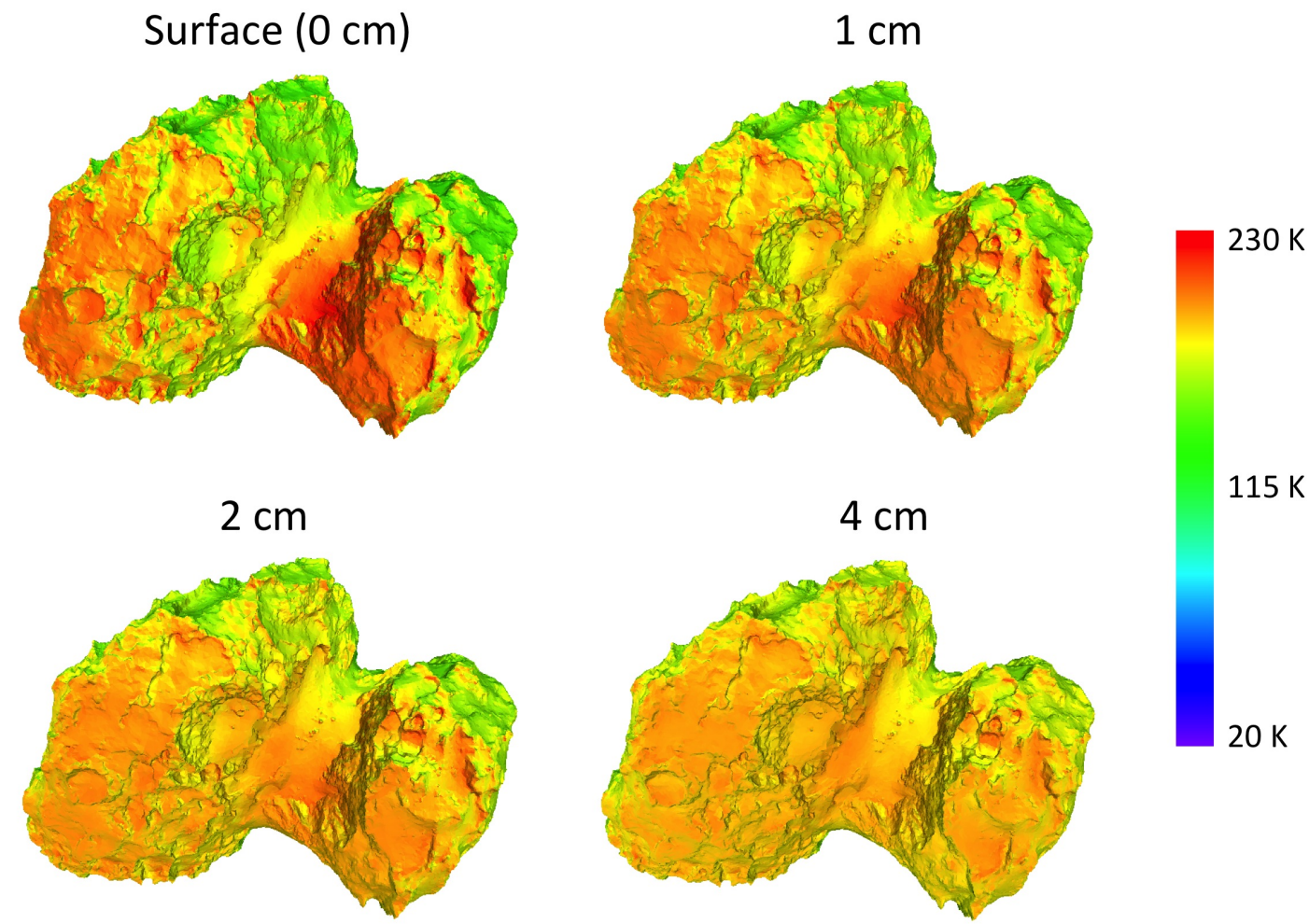

Figure 5.1: Temperature as a function of depth (on the surface and $1 \mathrm{~cm}, 2 \mathrm{~cm}$, and $4 \mathrm{~cm}$ below the surface) for a TI of $80 \mathrm{JK}^{-1} \mathrm{~m}^{-2} \mathrm{~s}^{-0.5}$, for September 15, 2014 (UT 00:00).

when an electric field is applied, respectively. Their values are relative to that of a vacuum, which is equal to $8.85 \times 10^{-12} \mathrm{~F} \mathrm{~m}^{-1}$. The real part of the permittivity is often referred to as the dielectric constant. The permittivity depends on the frequency, related to polarization mechanisms, composition, temperature, and bulk density of the material (Campbell and Ulrichs 1969, Mattei et al. 2014, Brouet et al. 2016).

For a given observed area and at a given wavelength of observation, the SBT is determined from the inverse Planck function taking into account the total spectral brightness intensity received from all facets covered by the MIRO beam. The spectral brightness intensity of a given facet varies as a function of the temperature profile of the subsurface and the permittivity of the material. It also depends on the emission angle, which refers to the orientation of the facet with respect to the main beam of the MIRO antenna. In this way, roughness at the scale of the shape model (of the order of metres) is treated in the interpretation of the MIRO measurements. A weighting function is applied to calculate the spectral brightness intensity of each facet. This function is based on an assumed Gaussian beam pattern for the MIRO instrument (Gulkis et al. 2007), where the centre of the beam contributes more strongly than the edges.

For one facet, the spectral brightness temperature intensity $B T_{v}$ as a function of the effective spectral brightness intensity $B T_{v, \text { eff }}$ and the surface emissivity, $\Upsilon$, is as follows

$$
B T_{v, \text { facet }}\left(\theta_{e}\right)=B T_{v, e f f}\left(\theta_{e}\right) \times \Upsilon\left(\theta_{t}, \theta_{e}\right)
$$



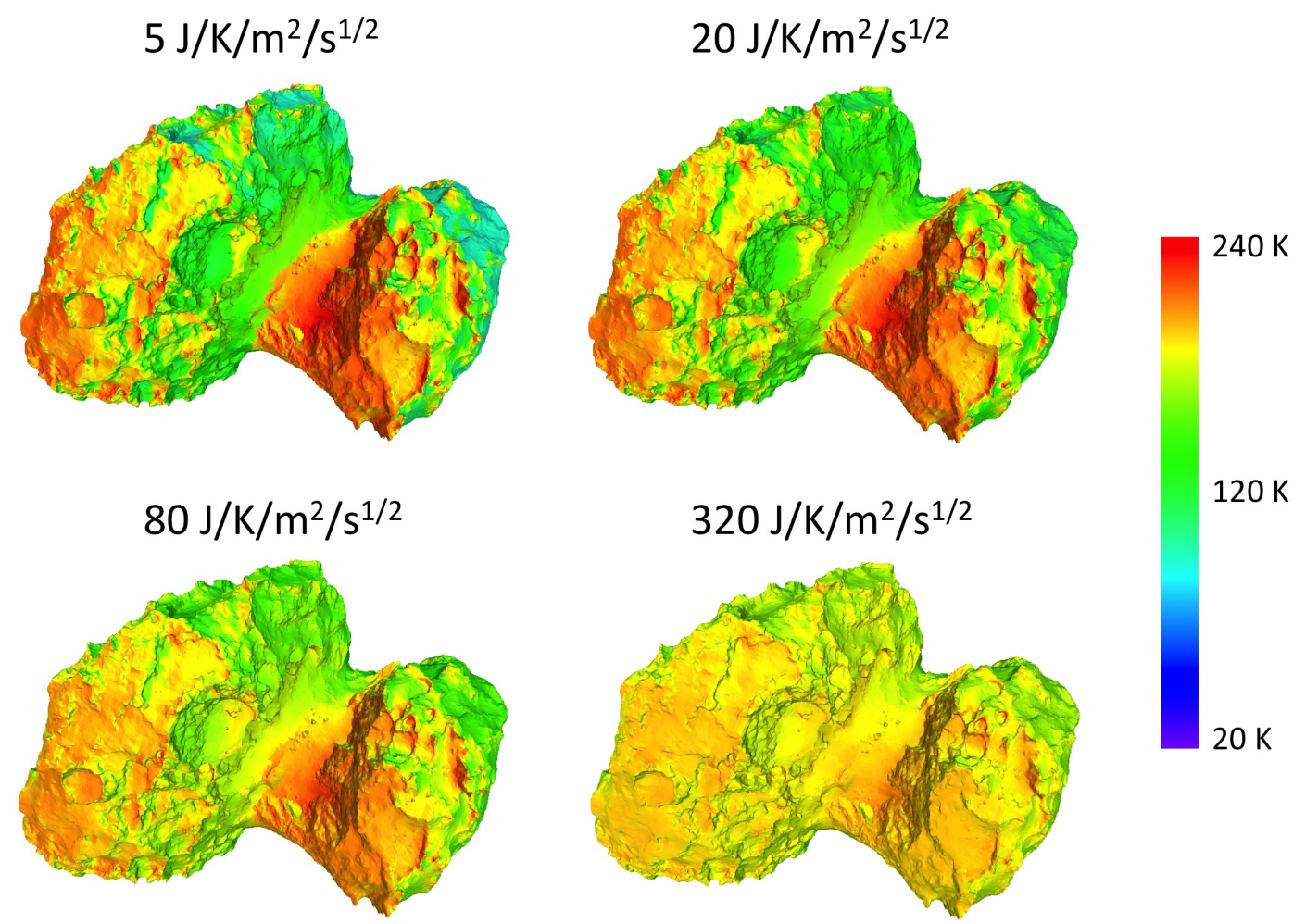

Figure 5.2: Temperature on the surface for various values of the thermal inertia in the range 5-320 $\mathrm{JK}^{-1} \mathrm{~m}^{-2} \mathrm{~s}^{-0.5}$, for September 15, 2014 (UT 00:00).

where $v$ is the frequency of the observation, $\theta_{e}$, the facet emission angle defined as the angle between the facet normal and the main beam of MIRO antenna during the observation, and $\theta_{t}$, the transmission angle in the subsurface layers of the facet. The transmission across a specular boundary is considered in order to compute the emissivity of the facet surface using the Fresnel reflectivity coefficients. The effective spectral brightness intensity is computed integrating the contribution of each subsurface layer, weighted by a radiative transfer function, which expresses the extinction of the spectral brightness intensity as it propagates towards the surface. The effective spectral brightness intensity computed for a given facet, assuming a scatter-free homogeneous layer under conditions of local thermodynamic equilibrium, is expressed as follows (Ulaby et al. 2014)

$$
B T_{v, e f f}\left(\theta_{e}\right)=\frac{1}{\sqrt{1-\sin ^{2}\left(\theta_{e}\right) / \epsilon^{\prime}} \times \delta_{e l}} \times \int_{0}^{H} B(v, T(z)) e^{-z /\left(\sqrt{1-\sin ^{2}\left(\theta_{e}\right) / \epsilon^{\prime}} \times \delta_{e l}\right)} d z,
$$

where $B(v, T(z))$, the Planck function for a given frequency of observation and temperature, $T(z)$, which varies with depth and is derived from the thermal model, and $\delta_{e l}$, the electrical skin depth of the layer at a given wavelength. The value $\mathrm{H}$ is the thickness of a layer equalling ten thermal skin depths. The electrical skin depth is inversely proportional to the imaginary part of the permittivity and proportional to the wavelength and the 
square root of the dielectric constant. It characterizes how deep an electromagnetic field can penetrate into the material.

Regarding the MIRO observations, we expect the submillimetre receiver to be sensitive to the thermal radiation integrated over the top subsurface of the layer and the millimetre receiver to be sensitive to the thermal radiation integrated over a greater depth. The electrical skin depth is constrained by a permittivity model based on experimental results obtained with lunar regolith samples reported in Olhoeft and Strangway (1975). The temperature dependence of the dielectric constant is considered to be linear (Calla and Rathore 2012, Brouet et al. 2015).

\subsubsection{Radiance model for VIRTIS data}

With the VIRTIS data, we focus our study on only two specific dates in Table 5.1: September 12 and September 15, 2014.

Fig. 5.3 shows the shape model of 67P rendered from the observing view of the spacecraft at those two epochs, and the radiance measured at $4.95 \mu \mathrm{m}$ by VIRTIS around that time. This wavelength was chosen because it is the longest in the VIRTIS range that is not directly at the detector edge and is outside certain spectral features that may be real or else caused by calibration issues (Filacchione et al. 2016). The measured radiance is a combination of directional thermal emission and reflected solar radiation. The observed areas cover the regions of Aten, Babi, Khepry, and Imhotep, as defined by El-Maarry et al. (2015). They are located in the northern hemisphere, at latitudes between 0 and +60 degrees and longitudes between 60 and 150 degrees. Because the data set covers several morphological regions, we expect significant variations in roughness values. Facets shared by the two datasets cover mainly the northern part of Imhotep and a small fraction of Aten.
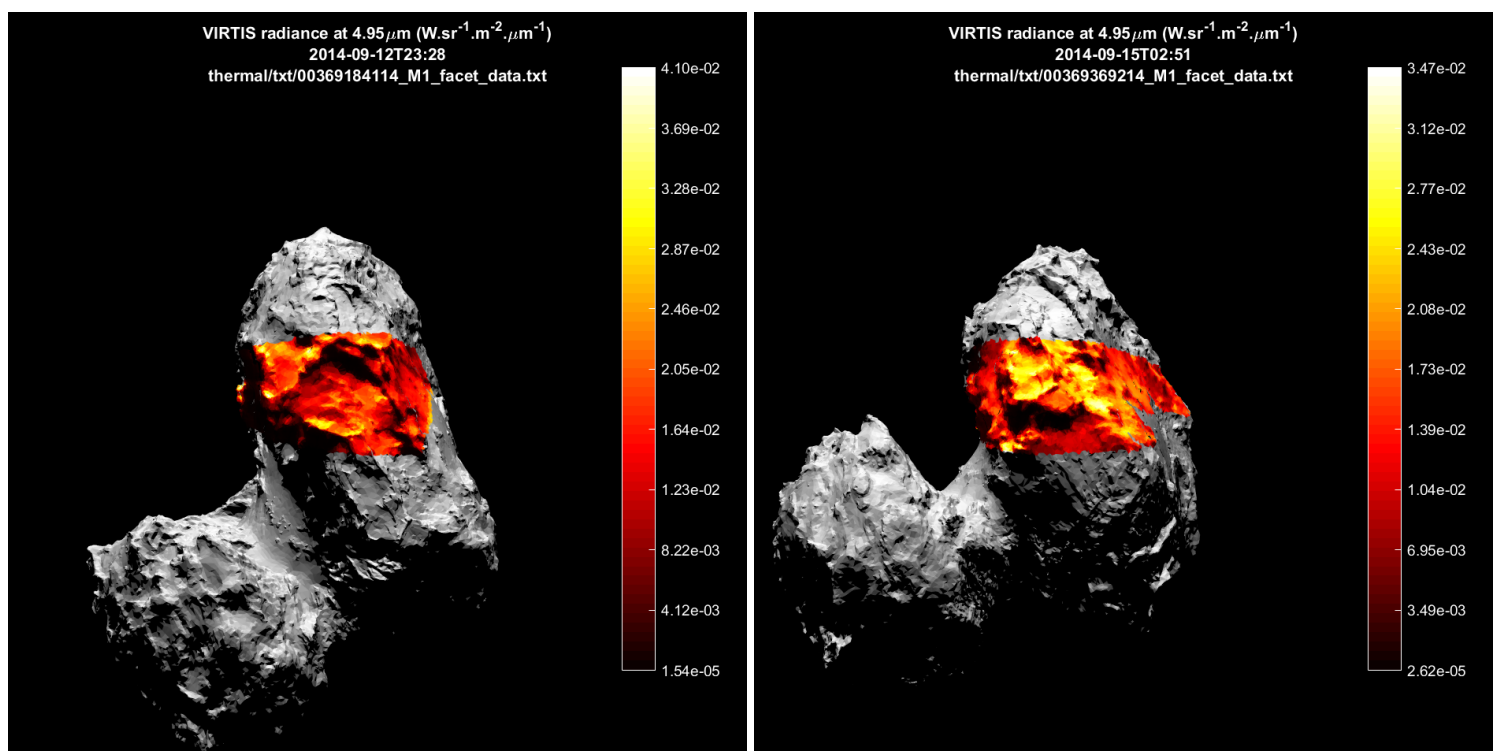

Figure 5.3: Radiance measurements from VIRTIS projected onto the shape model on 12 Sep 2014 (left) and 15 Sep. 2014 (right).

In order to compare data and model, we first convert the modelled surface tempera- 
ture into radiance, $I(\lambda, T)$, by combining reflected solar spectral radiance and emitted IR radiation as follows

$$
I(\lambda, T)=\alpha F(\lambda)+\epsilon B(\lambda, T)
$$

where $\alpha$ and $\epsilon$ are the constant geometric albedo and emissivity (respectively 0.05 and $0.95), F$ the solar flux modulated by a reflectance function (the Lommel-Seeliger Law), and $B$ the black body radiation at wavelength $\lambda$ and temperature $T$. We neglected the directionality of epsilon, which is affected by spatially unresolved surface roughness.

For a given facet on the shape model, the solar spectral radiance is given by Planck's law, heliocentric distance, and a photometric model. We chose the Lommel-Seeliger (Hapke 2012) correction, which depends only on incidence and emission angles. This equation is written as

$$
F(\lambda)=\frac{2 \mu_{0}}{\mu_{0}+\mu} \times\left(\frac{\pi R_{S u n}}{r_{h}}\right)^{2} \times \frac{2 h c^{2}}{\lambda^{5}} \frac{1}{e^{\frac{h c}{\lambda k \times 5778 K}}-1}
$$

where $h, c, k$ are Planck's constant, the speed of light, and the Boltzmann constant, respectively. The value $R_{S u n}$ is the sun radius, $r_{h}$ the heliocentric distance. The values $\mu_{0}$ and $\mu$ are the cosines of incidence and emission angle.

Finally, the emitted IR radiation is the Planck function at $T$ and $\lambda$, is

$$
B(\lambda, T)=\frac{2 h c^{2}}{\lambda^{5}} \times \frac{1}{e^{\frac{h c}{\lambda k T}}-1} .
$$

We applied our model to all facets covered by the VIRTIS data set, except those in shadows for which a more complex photometric model should be considered, accounting for multiple scattering. Shadows were calculated with a custom ray tracing engine ${ }^{1}$ widely used to simulate Rosetta images, and calibrated against observations.

\subsubsection{Importance of roughness}

In addition to TI and emissivity, it has been noted by many authors that roughness at spatial scales unresolved by the instrument plays an important role when determining the surface temperature of an airless body with a low TI, at many length scales (Kührt et al. 1992, Groussin et al. 2013, Keihm et al. 2012, Davidsson et al. 2015). First, the micro-topography (on centimetre scale) of the surface leads to re-radiation and multiple scattering of the incoming solar and thermal radiation, which can locally enhance or decrease the amount of energy received by forming heat traps or sinks. The temperature therefore varies on subpixel scales and we consequently observe a thermal radiation that cannot simply be represented by a single Planck function. One example is the case of craters, in which the shadowed wall can be heated up by radiation from the illuminated wall. Second, the illuminated fraction of the surface seen by the detector is influenced by the roughness, and so the energy received is a function of the relative position of the observer and the energy source.

Both issues limit the accuracy of temperature retrieval by remote sensing and must be accounted for. It should be noted that simulating accurately the roughness at the subpixel resolution of the OSIRIS (Keller et al. 2007, Optical, Spectroscopic, and Infrared Remote

\footnotetext{
${ }^{1}$ shapeViewer: www.comet-toolbox.com
} 
Imaging System) camera over a large surface is computationally far too expensive to be achieved in most cases. In practice, the surface temperature is first approximated with a classical Lambert surface. In a second step, the roughness is modelled by multiplying the Lambert temperature with a correction factor that is a function of wavelength, incidence angle, emission angle, projected phase angle, and a statistical description of the subpixel topography.

The subpixel topography can be represented in many ways, including fractal surface, coherent noise, and craters. Davidsson et al. (2015) showed that, for non-directional terrains (i.e. with facets orientated in all directions), it does not matter much in the final result which description is used. We adopted the approach proposed by Kührt et al. (1992), representing topographic variations with a set of spherically shaped depressions. The model is scale independent, and therefore our only free parameters are the area fraction covered by the craters and opening angle of the spherical segments. The opening angle refers to the centre of the corresponding sphere so an angle of $180^{\circ}$ creates a half-spherical depression.

In this study, we want to assess whether the roughness of surface of 67P can be estimated reliably from the combination of infrared measurements by the Rosetta VIRTIS instrument and a thermal model of the surface, described in sections 5.3 and 5.4.2, respectively. To do so, we assume that after accounting for the TI, any further discrepancy between model and data can be corrected by a factor that results solely from roughness effects. In this approach, we first derive a best TI for the observed area and then allow roughness to vary spatially in our model. We know all the geometric parameters for any facet of the shape model at any time, so we can use the roughness model to relate this correction factor to the area covered by the spherical segment depressions. This roughness estimation is then compared to OSIRIS images of the same region, as they have typically much higher spatial resolution than VIRTIS data. We finally assess how the spatial distribution of our retrieved thermal roughness (micrometre - millimetre scale) compares to the visual roughness (centimetre scale) in the images.

We therefore have three unknowns: TI and two roughness parameters (crater density and opening angle). Ideally combining three inputs would provide good constraints on the parameters: for instance the radiance in at least three wavelength channels for a given facet, or radiance measurements at the same wavelength but acquired with different geometries. However, since this is not always available in our dataset, we choose a less constrained approach, as outlined above.

\subsection{Results}

\subsubsection{MIRO results}

Fig. 5.4, 5.5, 5.6, 5.7, and 5.8 show the SBT as functions of the electrical skin depth for each of the thermal inertia values used in the thermal model, in the range 5 to 320 $\mathrm{JK}^{-1} \mathrm{~m}^{-2} \mathrm{~s}^{-0.5}$, for the five dates given in Table 5.2. As stated earlier, we constrain the TI when SBT intersects MBT for electrical skin depths less than $\sim 3.0 \mathrm{~m}$ and $\sim 1.0 \mathrm{~m}$ for the millimetre and submillimetre cases, respectively.

For example, in Fig. 5.4 in the millimetre channel, the SBT with $\mathrm{TI}=5 \mathrm{JK}^{-1} \mathrm{~m}^{-2} \mathrm{~s}^{-0.5}$ 

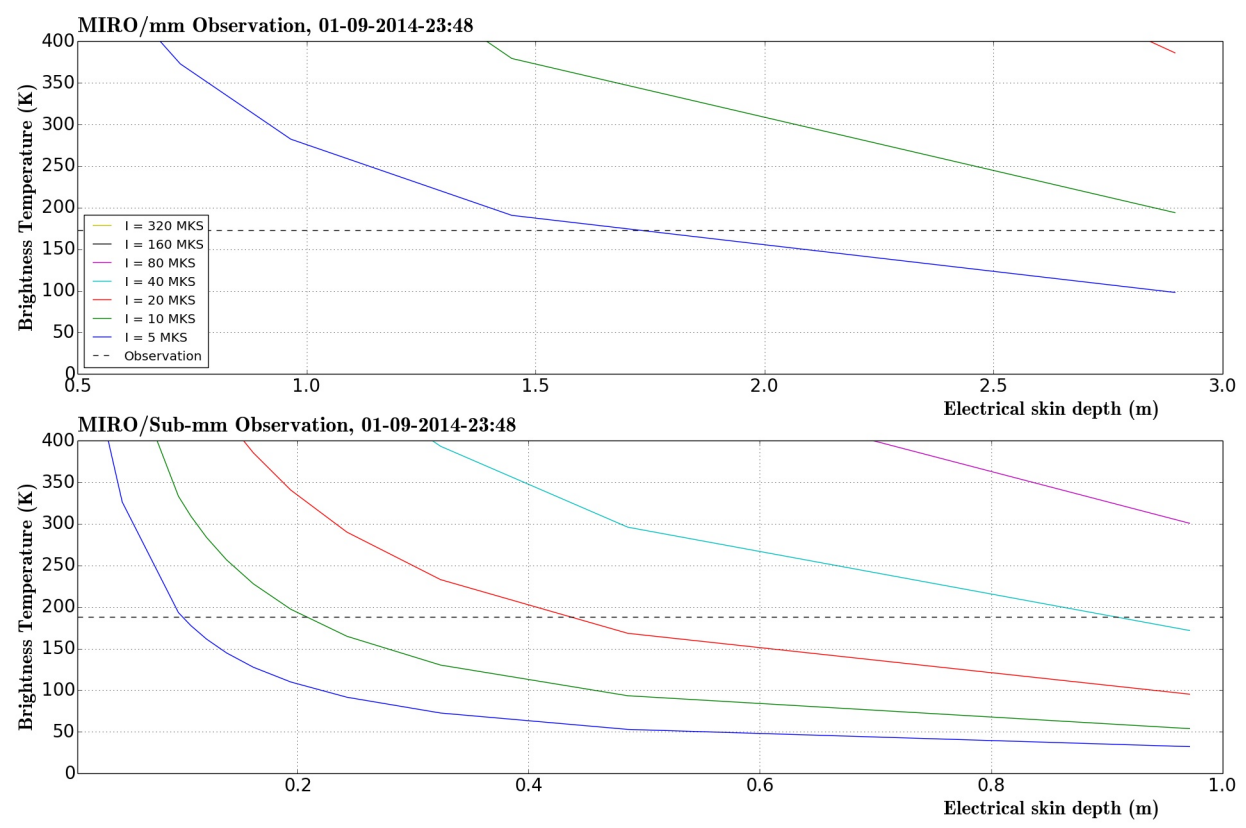

Figure 5.4: Simulated brightness temperatures (SBT; coloured lines), as functions of the electrical skin depth, for various values of TI in the range $5-320 \mathrm{JK}^{-1} \mathrm{~m}^{-2} \mathrm{~s}^{-0.5}$ (SI units). The black dotted lines represent the MBT from the millimetre and submillimetre receivers for September 1, 2014 at 23:48. The millimetre SBT curves with TI values less than $10 \mathrm{JK}^{-1} \mathrm{~m}^{-2} \mathrm{~s}^{-0.5}$ intersect the millimetre MBT line; and the submillimetre SBT curves with TI values less than $80 \mathrm{JK}^{-1} \mathrm{~m}^{-2} \mathrm{~s}^{-0.5}$ intersect the submillimetre MBT line.

intersects the MBT but TI $=10 \mathrm{JK}^{-1} \mathrm{~m}^{-2} \mathrm{~s}^{-0.5}$ does not. We hence derive that the TI in the millimetre channel for September 1 is $<10 \mathrm{JK}^{-1} \mathrm{~m}^{-2} \mathrm{~s}^{-0.5}$, as given in Table 5.2.

We choose not to further constrain the TI value by interpolating between the curves. Linear interpolation seems to be insufficient, so instead of devising a complex interpolation scheme, we quote the upper and lower bounds (where possible) from the discrete TI values, which initially went into the thermal model.

The MBT of the different observations performed with the millimetre receiver are consistent with a TI less than $80 \mathrm{JK}^{-1} \mathrm{~m}^{-2} \mathrm{~s}^{-0.5}$, while the value for September 1, 2014 implies that the TI should be less than $10 \mathrm{JK}^{-1} \mathrm{~m}^{-2} \mathrm{~s}^{-0.5}$. The results from the submillimetre receiver on September 1 and 2, 2014 are also consistent with a TI less than $80 \mathrm{JK}^{-1} \mathrm{~m}^{-2} \mathrm{~s}^{-0.5}$, but the other dates imply higher values of 160 and $320 \mathrm{JK}^{-1} \mathrm{~m}^{-2} \mathrm{~s}^{-0.5}$ are also possible for their observations. The results are compiled in the Table 5.2.

\subsubsection{VIRTIS results}

Disentangling TI from roughness effects is a complex problem for which we do not always have enough constraints. To reiterate, we attempt to fit first the TI as it relates directly to the physical properties of the material. We then add the surface roughness as a second parameter effectively describing which subpixel area contributes to the flux that reaches the detector. 

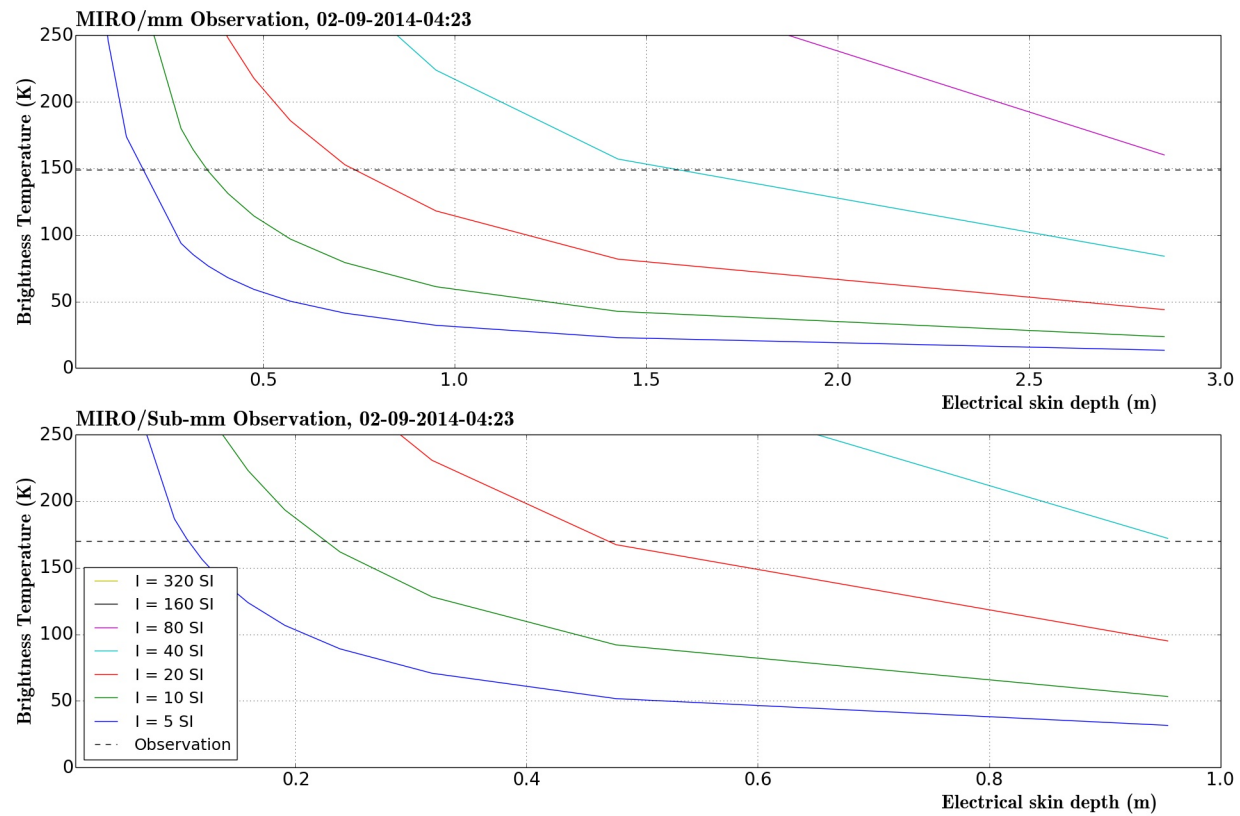

Figure 5.5: Same as Fig. 5.4 but for September 2, 2014. The results are given in Table 5.2 .
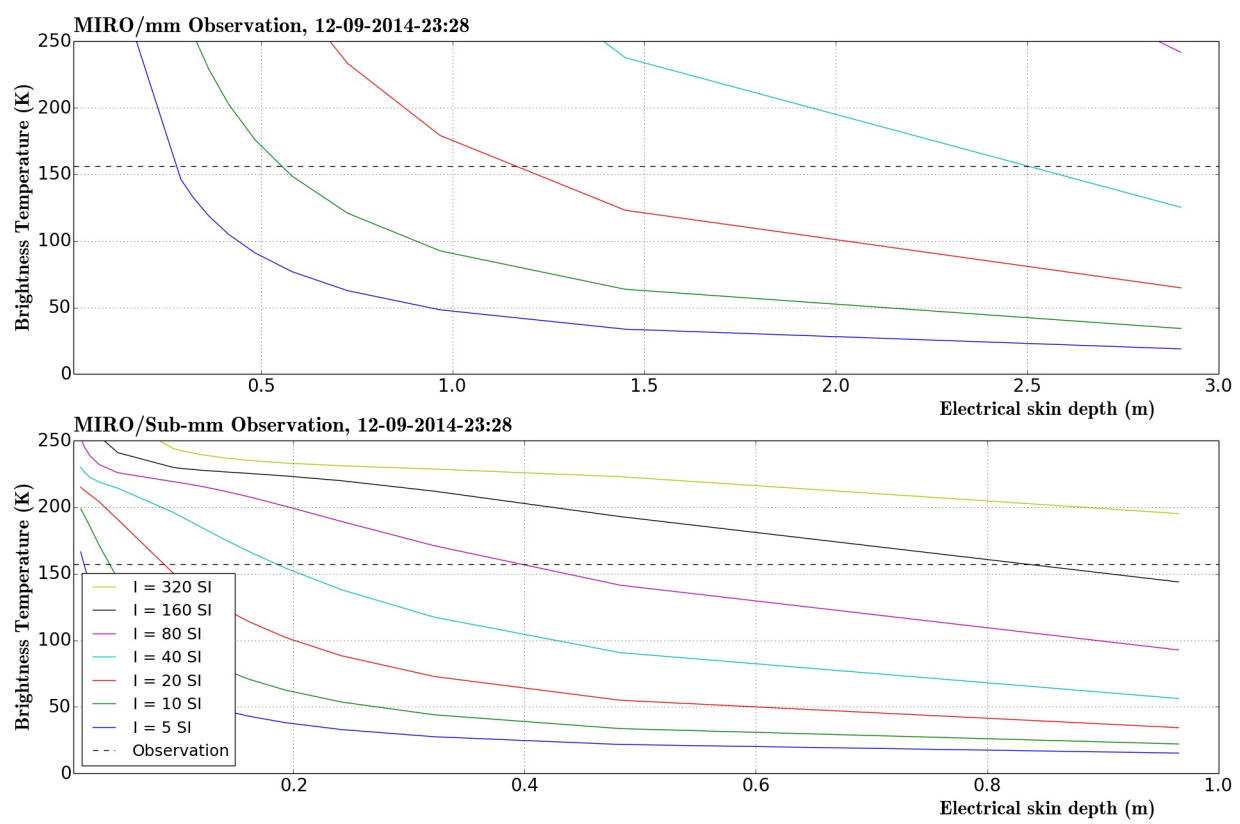

Figure 5.6: Same as Fig. 5.4 but for September 12, 2014. The results are given in Table 5.2 . 

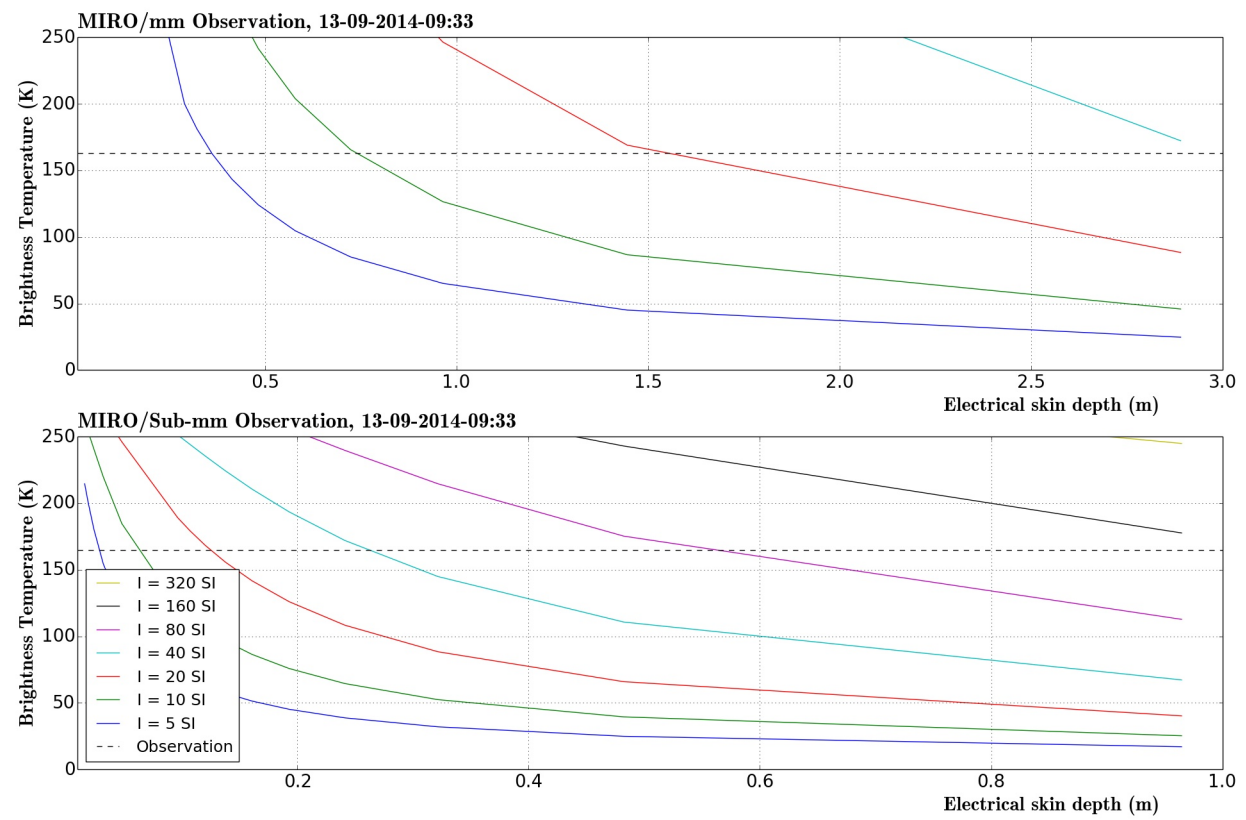

Figure 5.7: Same as Fig. 5.4 but for September 13, 2014. The results are given in Table 5.2 .
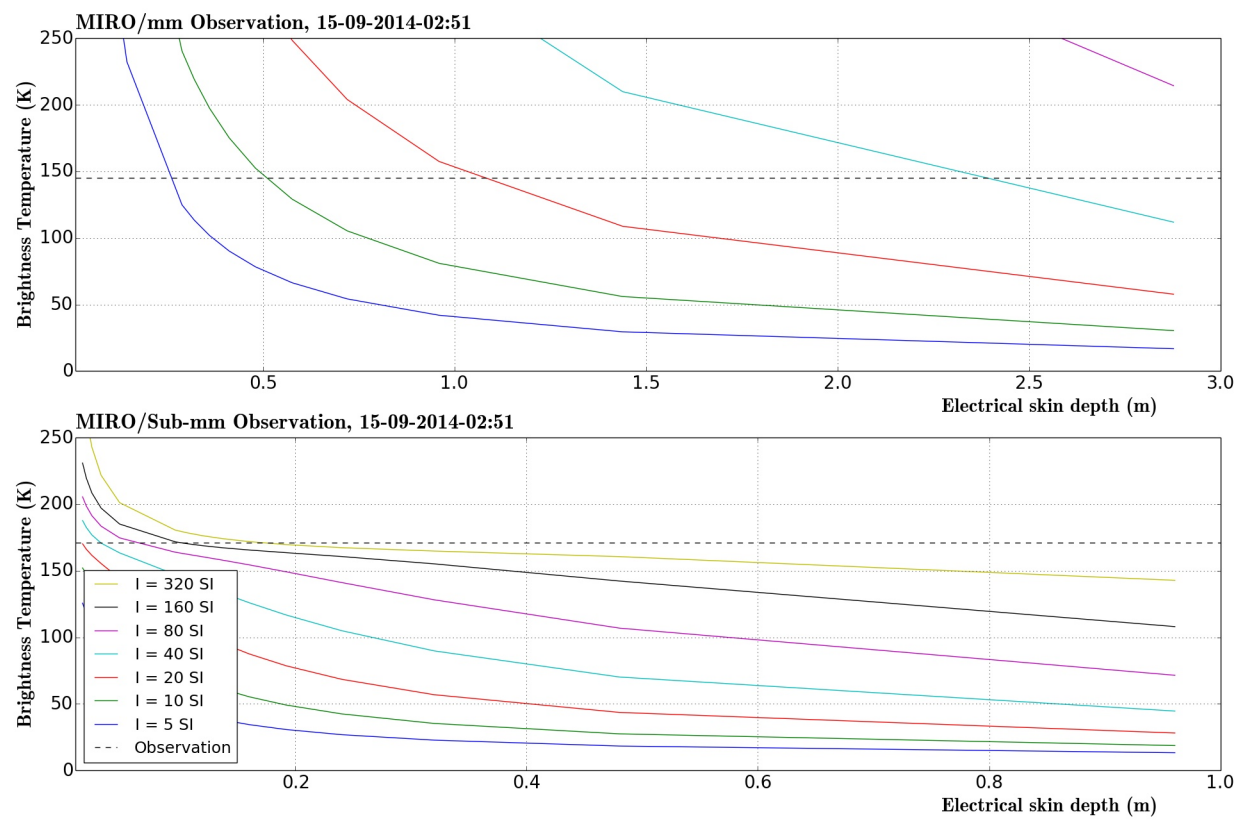

Figure 5.8: Same as Fig. 5.4 but for September 15, 2014. The results are given in Table 5.2 . 
5 Paper III - Thermal inertia and roughness

\begin{tabular}{ccccc}
\hline Date & Time & \multicolumn{2}{c}{ Thermal inertia $\left(\mathrm{JK}^{-1} \mathrm{~m}^{-2} \mathrm{~s}^{-0.5}\right)$} & Region \\
\hline & & from mm observation & from smm observation & \\
$01 / 09 / 2014$ & $23: 48$ & $<10$ & $<80$ & Seth \\
$02 / 09 / 2014$ & $04: 23$ & $<80$ & $<40$ & Aten \\
$12 / 09 / 2014$ & $23: 28$ & $<80$ & $5-320$ & Ash \\
$13 / 09 / 2014$ & $09: 33$ & $<40$ & $<160$ & Ash \\
$15 / 09 / 2014$ & $02: 51$ & $<80$ & $>20$ & Aten \\
\hline
\end{tabular}

Table 5.2: Range of possible local TI derived from the comparison between the SBT and the MBT for each MIRO observation.

Therefore, the first step in our inversion of roughness parameters is to determine which thermal inertia brings our model the closest to the measured radiance. We calculated the radiance for the seven values of TI investigated by the thermal model described above (TI $\left.=5,10,20,40,80,160,320 \mathrm{JK}^{-1} \mathrm{~m}^{-2} \mathrm{~s}^{-0.5}\right)$. We found that the modelled flux is almost always below the measured flux (see Fig. 5.9). As we increase TI, the average flux decreases. This is because higher TI means that facets must be illuminated for a longer time before reaching their maximum temperature. Our simulations show that a good fit is achieved for a TI larger than $80 \mathrm{JK}^{-1} \mathrm{~m}^{-2} \mathrm{~s}^{-0.5}$. We therefore use this TI value as a lower limit in our analysis. Higher values lead to an equally good fit, suggesting that TI is not the main reason for the dispersion we observe in Fig. 5.9, although the fit is slightly worse for TI values of 160 and $320 \mathrm{JK}^{-1} \mathrm{~m}^{-2} \mathrm{~s}^{-0.5}$ in the case of September $15,4.75 \mu \mathrm{m}$.
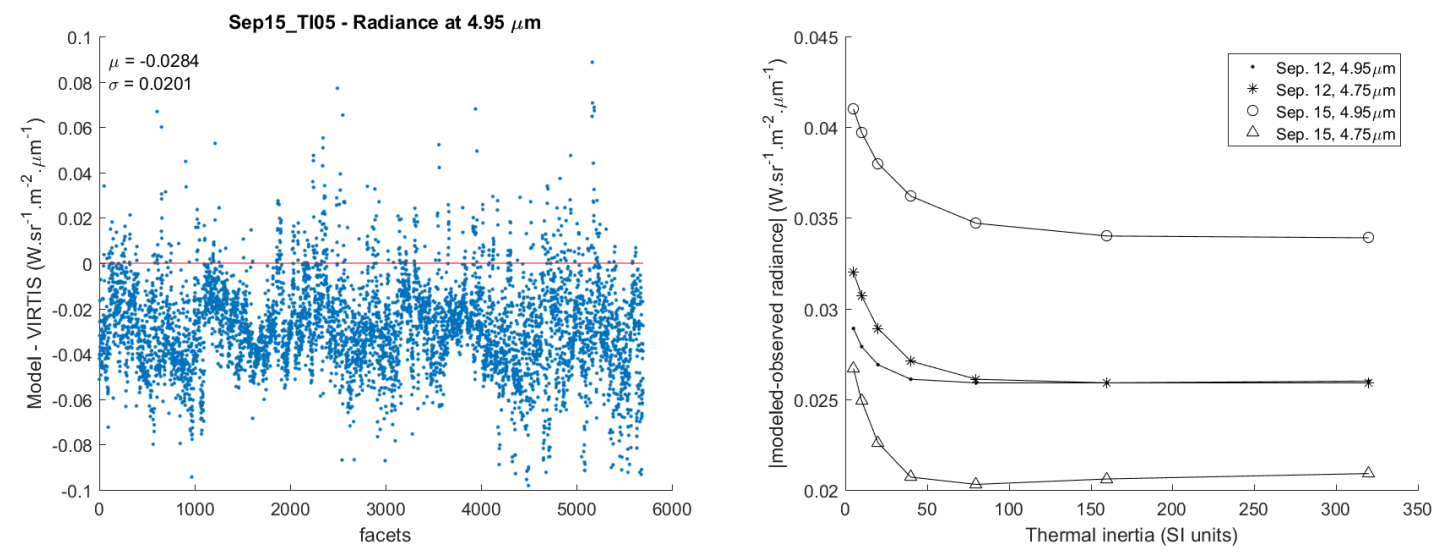

Figure 5.9: Left: Example of modelled radiance minus observed radiance at $4.95 \mu \mathrm{m}$ on September 15, 2014 for $\mathrm{TI}=5 \mathrm{JK}^{-1} \mathrm{~m}^{-2} \mathrm{~s}^{-0.5}$. Increasing the TI makes the plot more symmetrical around zero. Right: Mean value of model minus observation as a function of TI for two epochs and two wavelengths. We observe that increasing the TI beyond 80 $\mathrm{JK}^{-1} \mathrm{~m}^{-2} \mathrm{~s}^{-0.5}$ does not improve the fit, and slightly worsens it for the case September 15 , $4.75 \mu \mathrm{m}$. We therefore use this TI value as a lower limit in our analysis.

Having selected $\mathrm{TI}=80 \mathrm{JK}^{-1} \mathrm{~m}^{-2} \mathrm{~s}^{-0.5}$ as our reference TI, we then postulate that the remaining discrepancy between model and observation is caused by the surface roughness. Following the approach of Kührt et al. (1992), we define the corrected flux for a rough terrain as $I_{r}=c_{r} \times I$, with $c_{r}$ a function of incidence and emission angles defined 
by the shape model facet normals, projected phase, crater density, crater opening angle, and wavelength. The projected phase, or azimuth, is the angle between the observer direction and solar direction vectors projected onto the facet surface. In this approach, the only free parameters are the crater density and the crater opening angles. Our test cases show that the influence of opening angle is less important than that of the crater density for the wavelengths considered. Therefore, we fix the opening angle to a value of 180 (which leads to maximal $c_{r}$ ) and find the crater density that minimizes the quantity $I_{r}-I_{m}$ : the difference between the calculated radiance, $I_{r}$, and the VIRTIS measured radiance, $I_{m}$. The crater density is allowed to vary between 0 and $\frac{\pi}{2 \sqrt{3}}$, the maximum density for packing equal circles on a two-dimensional plane. This approach effectively gives us a lower bound for the crater density on each facet in the region of interest.

We note that in principle, one should find the best parameters by varying both TI and roughness in parallel when getting the minimum least-squares fit. However, this is a large parameter space and running the complete inversion goes beyond the scope of this work.
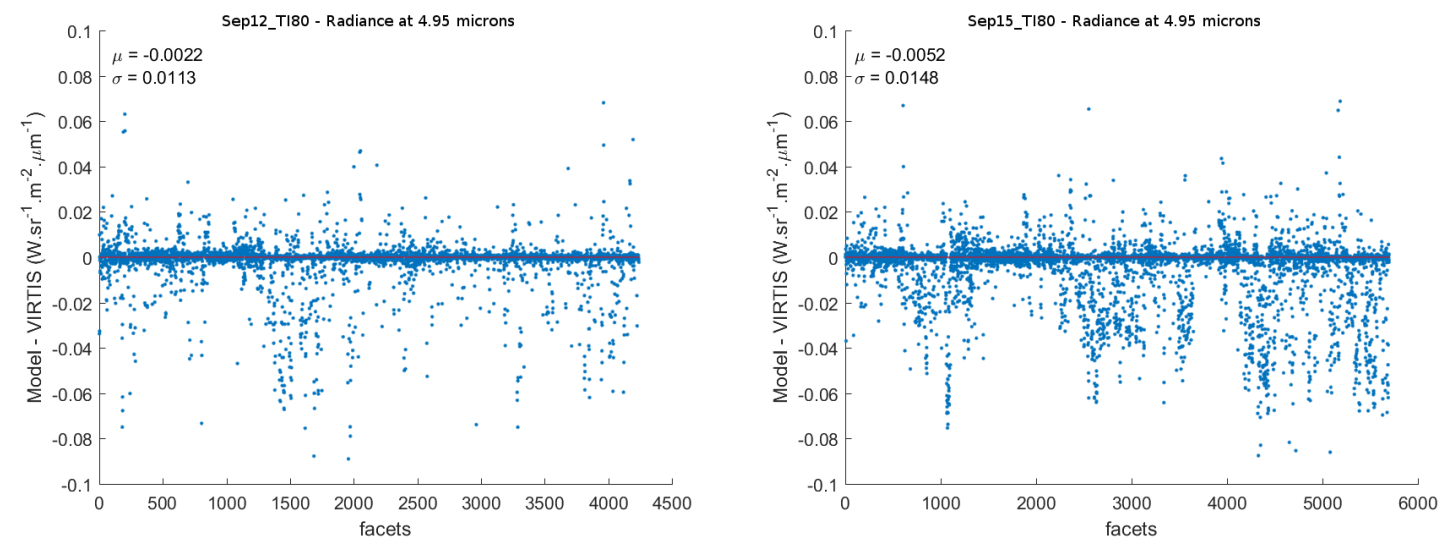

Figure 5.10: Difference between modelled rough radiances and VIRTIS measurements for September 12, 2014 (left) and September 15, 2014 (right). Although we still observe some scattering, the dispersion is far smaller than when not considering roughness, as in Fig. 5.9.

Fig. 5.10 shows an example of the corrected radiances for two different dates. The most remarkable effect is the strongly reduced scattering of our data points with respect to Fig. 5.9, indicating a very good agreement with the observations. The residual discrepancies can be explained by the fact that we have used a relatively coarse shape model (124938 facets, $20 \mathrm{~m}$ resolution) and the same value of TI for all facets. A finer spatial resolution for both parameters would certainly improve the fits, as would a more advanced roughness model and spatially varying emissivity. In addition, it should be noted that VIRTIS measurements are not instantaneous, but require up to 20 minutes in this work, during which the viewing geometry changes as the comet rotates and the spacecraft moves. This also affects our inversion.

Figures 5.11 and 5.12 illustrate our results; the retrieved crater density is projected on the shape model. One can see that the distribution of densities is not random, but rather organized in regions whose distribution resembles the morphological regions derived from optical data alone. 

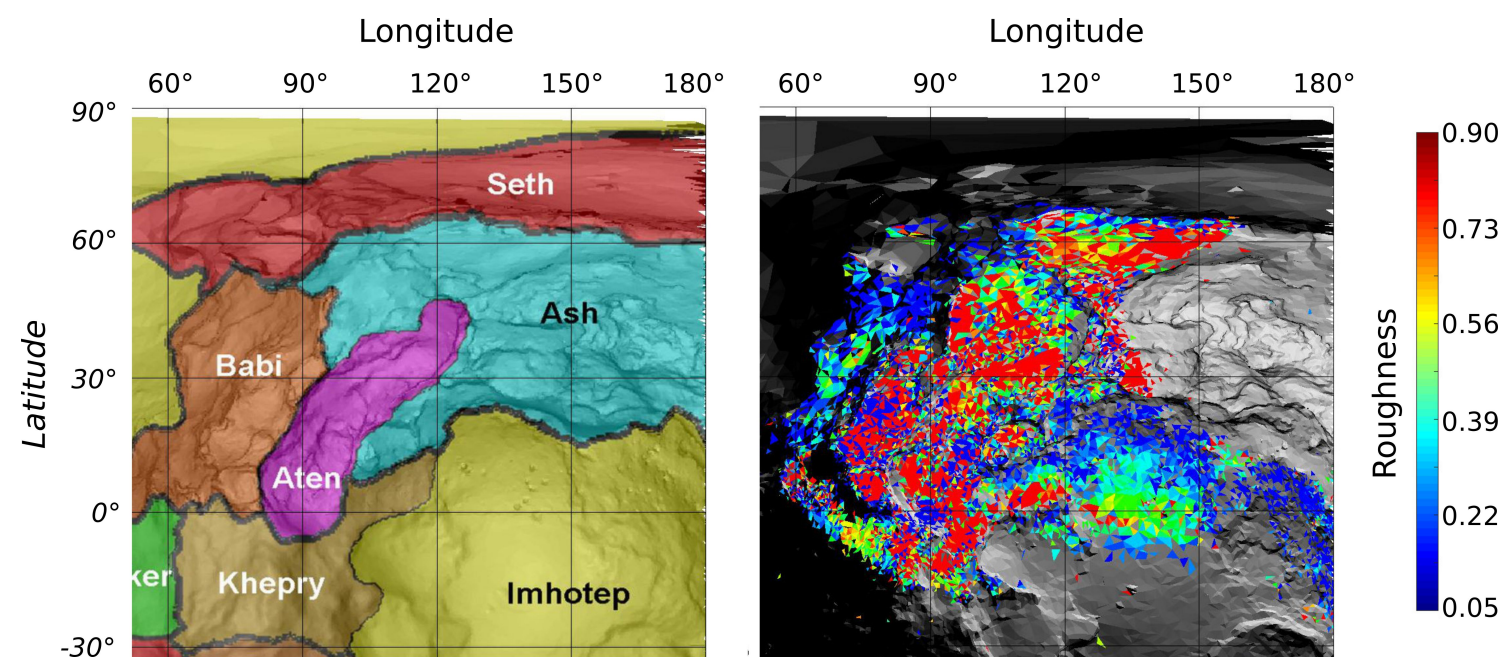

Figure 5.11: Left: Regional map of the comet from El-Maarry et al. (2015) with morphological units. Right: Roughness parameter, i.e. the density of the half-spherical depressions projected onto the shape model. The density value is represented by the colour bar on the right.
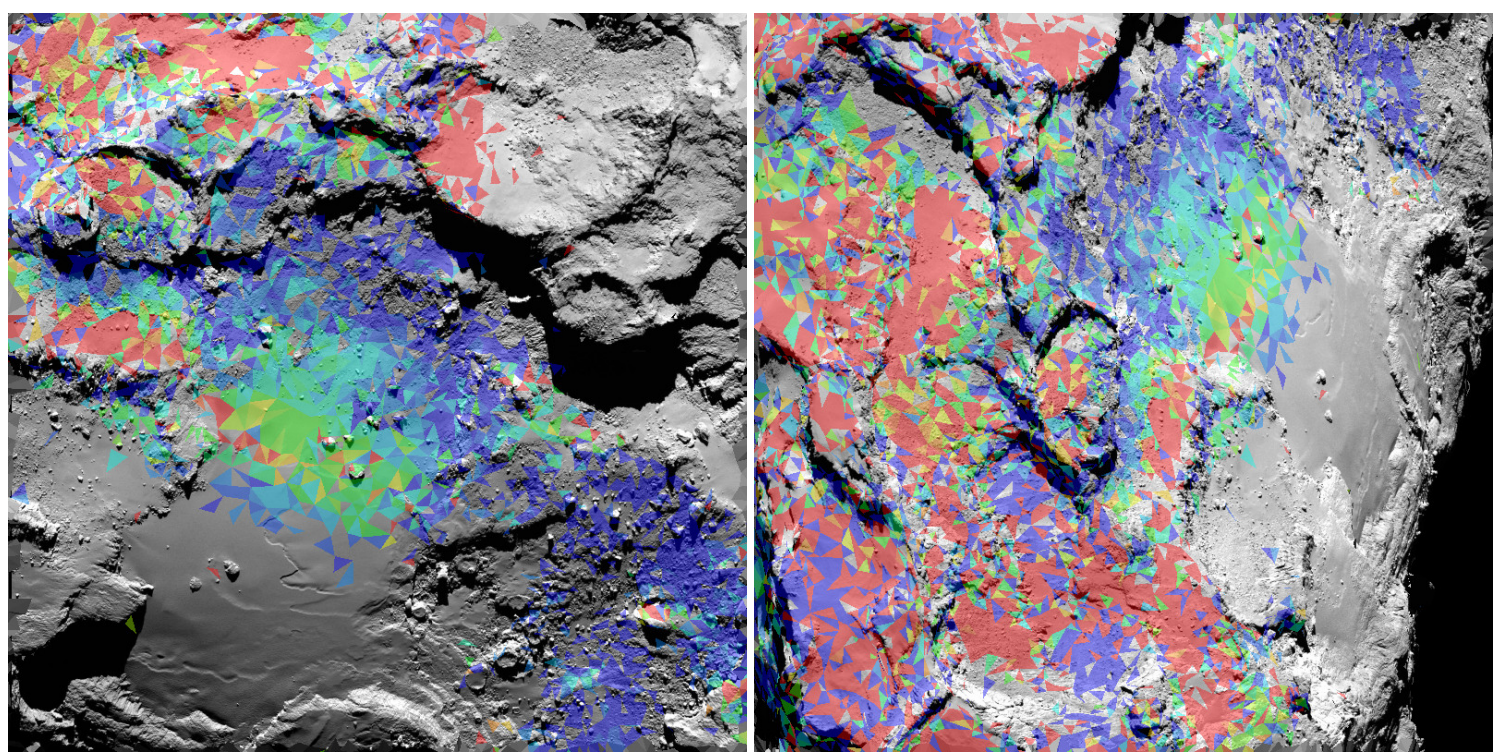

Figure 5.12: Half-spherical depression density overlaid on OSIRIS images, 3D projected onto the shape model with the same colour scheme as in Fig. 5.11. The images are centred on approximately $130^{\circ}$ longitude and $10^{\circ}$ latitude. One can see that areas with similar morphologies share similar roughness. Visually smooth terrains (at the scale of OSIRIS data, i.e. $75 \mathrm{~cm} / \mathrm{px}$ ) can display high thermal roughness, indicating that the spatial scale of this parameter is far below the resolution of our images.

We find a similar crater density for regions with a similar morphology, although this retrieved parameter may appear sometimes counter-intuitive. For instance, when looking at morphology alone, the Imhotep plain appears to be separated in two areas with different textures. The northern part is rougher with many metre-size boulders, while the southern area is very smooth and has topographic variations that are basically undetectable at the 
best OSIRIS resolution $(<50 \mathrm{~cm} / \mathrm{px})$. Interestingly, our roughness inversion also discriminates between these two subregions, thus helping to validate our method, but the area with highest crater density is the subregion that appeared to be the smoothest in OSIRIS images.

Although this may seem surprising at first, it is actually explained by the fact that the roughness that plays a role for IR measurements is simply at a much lower scale than topographic variations. Taking this into account, it seems reasonable to postulate that consolidated terrain is likely to show uniform textures on a centimetre scale, while a visually smooth terrain may be covered in pebbles and dust aggregates that form a very rough surface at small scales.

This result is consistent with several observations from the Rosetta lander Philae. Images acquired by the ROLIS camera during the descent to the Agilkia region on November 12,2014 show that a region that appeared as a smooth dusty plain in OSIRIS images is actually covered by centimetre-sized pebbles when observed at higher resolution (Mottola et al. 2015). This region has a very granular appearance as seen by ROLIS (Rosetta Lander Imaging System) and may be a result of physical processes occurring on the comet such as airfall of small material or talus deposits. The same may be true for the Imhotep dust plain, as revealed by our thermal analysis. On the other hand, CIVA (Comet Infrared and Visible Analyser) images obtained at Abydos after landing show that the consolidated terrain, although very rough on a metre-size scale, display flat and smooth facets at centimetre to decimetre scale (Bibring et al. 2015). Flat and smooth morphologies could be a result of a variety of process, including the disaggregation of boulders by complete erosion to create rounded structures, or the fragmentation of hard consolidated material to reveal flat surfaces (Mottola et al. 2015). Further study is required to link the measured roughness to these morphological processes.

\subsection{Discussion and conclusions}

\subsubsection{Thermal inertia}

All of the results obtained from the MIRO millimetre observations imply that the TI has a low value, $<80 \mathrm{JK}^{-1} \mathrm{~m}^{-2} \mathrm{~s}^{-0.5}$. The result from September 1 even implies that it should be $<10 \mathrm{JK}^{-1} \mathrm{~m}^{-2} \mathrm{~s}^{-0.5}$ in the Seth region. For this date, the submillimetre channel gives a higher upper bound of $80 \mathrm{JK}^{-1} \mathrm{~m}^{-2} \mathrm{~s}^{-0.5}$, similar to other results.

Constraining the TI from the subsurface temperature observations is sometimes difficult, however. For the measurements on September 12, 2014 and September 15, 2014, the submillimetre observation cannot constrain the TIso well $\left(5-320 \mathrm{JK}^{-1} \mathrm{~m}^{-2} \mathrm{~s}^{-0.5}\right.$ and $>20 \mathrm{JK}^{-1} \mathrm{~m}^{-2} \mathrm{~s}^{-0.5}$, respectively) based on the assumed dielectric model. The results from the millimetre observations on these two dates are better constrained however, giving an upper bound of $80 \mathrm{JK}^{-1} \mathrm{~m}^{-2} \mathrm{~s}^{-0.5}$.

In comparison, VIRTIS work implies a best-fitting value of $80 \mathrm{JK}^{-1} \mathrm{~m}^{-2} \mathrm{~s}^{-0.5}$ across the observed Aten, Babi, Khepry, and Imhotep regions. The VIRTIS value was calculated over a much larger area than the MIRO values as the viewing area of VIRTIS is larger than the MIRO beam area. However, this does not seem to make a difference. With the VIRTIS data restricted to a subset containing only the facets viewed by the MIRO beam, 
the VIRTIS result remains the same; a similar thermal inertia value is predicted.

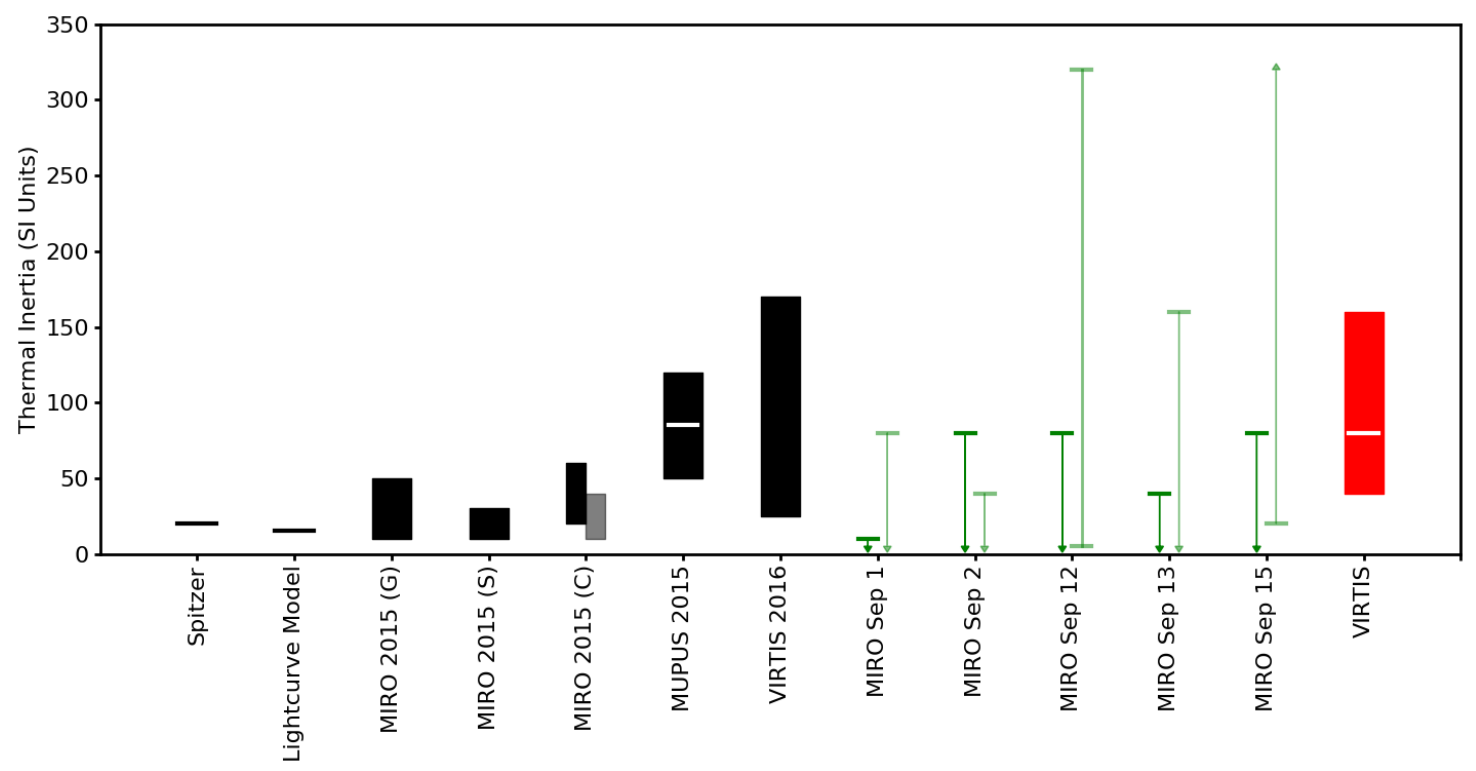

Figure 5.13: Summary of measured TIs (units: $\mathrm{JK}^{-1} \mathrm{~m}^{-2} \mathrm{~s}^{-0.5}$ ) from a variety of sources. Measurements in black represent previous published works and coloured bars represent this work. From left to right, these measurements represent the TI from: (a) Spitzer observations (Lamy et al. 2008); (b) a thermophysical model applied to light curve observations (Lowry et al. 2012); (c) MIRO observations by Gulkis et al. (2015); (d) MIRO observations by Schloerb et al. (2015) (e) MIRO observations by Choukroun et al. (2015), with TI from the millimetre channel in black and TI from the submillimetre channel in grey; (f) MUPUS observations, with average represented in white (Spohn et al. 2015); (g) VIRTIS observations (Leyrat et al. 2016); (h) MIRO observations from this work in Seth region, with TI from the millimetre channel in dark green and TI from the submillimetre channel in light green (horizontal lines represent upper or lower bounds); (i) MIRO observations from this work in Aten region; (j) MIRO observations from this work in Ash region; (k) MIRO observations from this work in Ash region; (1) MIRO observations from this work in Aten region; and (m) VIRTIS observations from this work; the best-fitting value is indicated in white.

In Figure 5.13, we compare our measurements for the TI from this work with previous observations. Our measurements for the TI from MIRO are consistent with past MIRO measurements of the TI (Gulkis et al. 2015, Schloerb et al. 2015, Choukroun et al. 2015 ) and there is also some overlap with the lander observation (Spohn et al. 2015). The measured VIRTIS TI is in good agreement with the MUPUS value, which was also determined from an infrared instrument: MUPUS-TM operates at $5-25 \mu \mathrm{m}$ and VIRTIS observes at 2-5 $\mu \mathrm{m}$. In addition, further VIRTIS calculations have yielded a TI in the range $25-170 \mathrm{JK}^{-1} \mathrm{~m}^{-2} \mathrm{~s}^{-0.5}$ (Leyrat et al. 2016). Neglecting spatial and depth variations, a bulk TI value in the range $30-50 \mathrm{JK}^{-1} \mathrm{~m}^{-2} \mathrm{~s}^{-0.5}$ would fit all of the measurements from Rosetta instruments, except for the MIRO millimetre measurement made here on September 1 . This value is similar to the early TI estimates from Lamy et al. (2008) and Lowry et al. (2012), which are much lower than most of the Rosetta instrument measurements. 
Taken together, the Rosetta measurements are consistent with a low TI $\left(\leq 170 \mathrm{JK}^{-1} \mathrm{~m}^{-2} \mathrm{~s}^{-0.5}\right)$, and there is notable overlap in the error bars of these results.

\subsubsection{Roughness}

In the MIRO analysis, roughness is considered on the scale of the horizontal shape model resolution because the angle between each facet normal of the shape model and the direction to the spacecraft is taken into account when computing the SBT. We considered adding subfacet roughness into the MIRO analysis but this would be computationally intensive. In addition, the uncertainty on the TI is larger than the expected uncertainty caused by subfacet roughness because of the valid range of values considered for the electrical skin depth.

The VIRTIS analysis showed that the roughness could be considered on subpixel scales that are below the resolution of the OSIRIS images. While work remains to be done to define the surface roughness properly, our approach shows that one can use IR radiance measurements and a simplified roughness model to separate the surface into regions of different properties. This study raises the prospect of consistently mapping the full surface at various epochs, in particular in areas like Imhotep in which significant changes have been detected at large and small scales over the course of the Rosetta mission (Groussin et al. 2015). We conclude that the roughness below the resolution of the images plays a key role in interpreting thermal infrared observations, and that one cannot use the visible images to estimate the surface roughness at this scale, since the smallscale (IR wavelength) roughness is not well correlated with the roughness on the large scale (visible wavelength).

This work demonstrates the importance of comparative studies of results from different instruments. By combining observations from the MIRO, VIRTIS, and OSIRIS instruments on Rosetta, we have gained new insights into the small-scale roughness of the surface and found more evidence for a low TI of the comet nucleus. 



\section{Future work}

\subsection{Introduction}

Ever since Whipple (1950), we have understood comets as small solar system bodies made from volatile ices and refractory material. As they fly close to the sun, comets heat up as a result of the increase in received flux and sublimate their volatile components, forming a tenuous gas atmosphere around the nucleus called the coma.

One of the initial goals of the MIRO instrument was to use the acquired spectral lines to study the evolution and development of the inner coma (Gulkis et al. 2007). The inner coma is unobservable from ground-based telescopes, and in situ instruments can only probe this region if they are in it. MIRO offers a unique opportunity to observe this region around comet 67P/Churyumov-Gerasimenko remotely, and throughout the duration of the Rosetta mission.

Spectral line inversion has long been applied to Earth for meteorological purposes to obtain temperature profiles in the atmosphere (for example, King (1952)) and now more recently to other solar system bodies (Hartogh et al. (2010a) for Mars; Lellouch et al. (2015) for Uranus and Neptune; Thelen et al. (2018), Rengel et al. (2014), Bauduin et al. (2018) for Titan). As a result of the Rosetta mission, this can now be applied to a comet for the first time and the parameters of interest in the coma are the gas kinetic temperature, the expansion velocity (always given as the velocity of the gas towards the spacecraft) and the number density of water molecules. The $\mathrm{H}_{2}{ }^{16} \mathrm{O}$ line is optically thick in the MIRO observations whilst the $\mathrm{H}_{2}{ }^{18} \mathrm{O}$ line is optically thin (Gulkis et al. 2015) and the different opacities of each line give sensitivity to different altitudes of the coma.

Currently, the inner coma region is typically modelled with parametrised functions (Lee et al. 2015, Marshall et al. 2017, Hansen et al. 2016) but the actual behaviour may be far more irregular. Inverting the observed spectral lines can reveal the turbulent dynamics of cometary activity which parametrised profiles cannot capture. Activity is well observed but unfortunately not well understood. For instance, it is not always known which molecular species is driving the outgassing at a given time, how much of the surface is active, how the activity distribution changes, or even how the non-volatile components of the comet are lifted from the surface. By thoroughly characterising the inner coma, we can hope to address some of these open questions as the behaviour at the surface may be reflected in the dynamics of the coma.

The aim of this chapter is to introduce the application of inversion techniques to the MIRO spectra which can then be used in future analysis of the data. In the next section, I will describe the method of iterative regularised inversion and in Section 6.3.1, I will apply this to a synthetic case study to test the accuracy of the profile retrieval. This will 
then be applied to MIRO data in Section 6.3.2.

\subsection{Method}

In this section, I introduce the method which will be used for the inversion of the MIRO data. The method uses a regularised iterative inversion process called Optimal Estimation (Rodgers 2000) which starts from an a priori profile to constrain the solution. A single set of line of sight profiles for gas temperature, expansion velocity and number density are retrieved from a single pair of $\mathrm{H}_{2}{ }^{16} \mathrm{O}$ and $\mathrm{H}_{2}{ }^{18} \mathrm{O}$ observations.

\subsubsection{Creating the a priori profiles}

The first step is to create the a priori profiles from which the inversion will be constrained. Since there is very little prior knowledge available for the conditions of the inner coma, this is done by utilising the atmospheric information in the nadir water spectral lines:

- The continuum temperature, $T_{c}$, measured by MIRO can act as proxy for the near surface temperature in the coma. In reality this is a sub surface temperature which may be different to the temperature of the gas in the lowest coma altitudes

- The temperature of the flat saturated $\mathrm{H}_{2}{ }^{16} \mathrm{O}$ line core, $T_{s}$, gives an estimate for the coma temperature closest to the spacecraft altitudes

- The Doppler shift of the $\mathrm{H}_{2}{ }^{18} \mathrm{O}$ line, $v_{\text {exp }}$, can be used in the a priori as the projected velocity in the coma around altitudes close to the spacecraft.

- The line area ratio between the $\mathrm{H}_{2}{ }^{16} \mathrm{O}$ and $\mathrm{H}_{2}{ }^{18} \mathrm{O}$ lines can determine an estimate for the column density, $N$, as described in Marshall et al. (2017) which can then be used to set the integrated area under the density profile.

This information from the spectral line helps to create a priori profiles for the temperature, velocity and density profiles. The temperature and velocity a priori profiles are spline functions which are schematically represented in Figure 6.1. Both spline functions have 5 knots given in Table 6.1 .

The first two knots are at $5.5 \mathrm{~m}$ and $100 \mathrm{~m}$ and take temperature and velocity values $T_{g r}$ and $v_{g r}$, respectively. These are chosen within the limits $T_{c}-10 \mathrm{~K} \leq T_{g r} \leq T_{c}+10 \mathrm{~K}$ and $0 \mathrm{~km} / \mathrm{s} \geq v_{g r} \geq-0.4 \mathrm{~km} / \mathrm{s}$. $T_{c}$ is measured from the MIRO spectra as mentioned above. One of the major constraints when creating the a priori profiles is the lack of information about the velocity close to the nucleus. There is not much information about the velocity flow in this region in the MIRO spectra so our a priori profiles take a range of near-nucleus velocities from $0-0.4 \mathrm{~km} / \mathrm{s}$. This range of values covers possible values for

the most probable thermal speed, $v_{p}=\sqrt{\frac{2 R T_{c}}{M}}=\sqrt{922 T_{c}}$ where $R=8.3 \mathrm{~J} \mathrm{~mol}^{-1} \mathrm{~K}^{-1}$ is the gas constant, $M=0.018 \mathrm{~kg} \mathrm{~mol}^{-1}$ is the molar mass of water, and $T_{c}$ is assumed to be in the range $0-200 \mathrm{~K}$.

The fifth knot is at the altitude of the spacecraft $H_{s / c}$ and the temperature and velocity in this region, $T_{s / c}$ and $v_{s / c}$, respectively, are chosen within the limits $T_{s}-15 \mathrm{~K} \leq T_{s / c} \leq$ $T_{s}+15 \mathrm{~K}$ and $v_{\text {exp }}+0.1 \mathrm{~km} / \mathrm{s} \geq v_{s / c} \geq v_{\text {exp }}-0.1 \mathrm{~km} / \mathrm{s}$. 


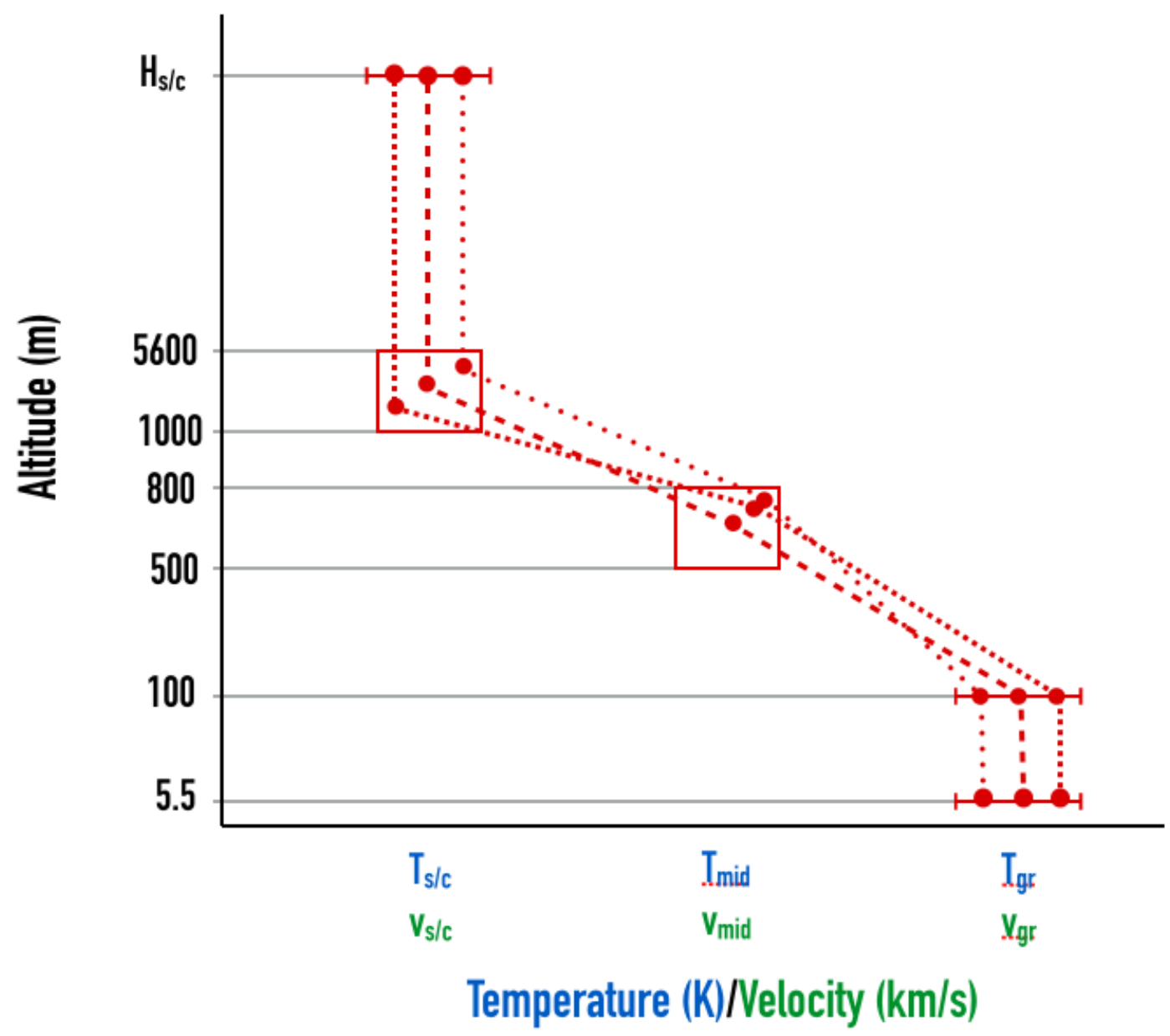

Figure 6.1: Schematic depiction of the creation of the a priori profiles for temperature and velocity. The a priori are spline functions with five knots (red circles). The co-ordinates of each knot are randomly generated from the intervals given in Table 6.1. The dashed lines show how different a priori profiles can be constructed where $T_{g r}$ and $v_{g r}$ are the temperature and velocity close to the nucleus, $T_{s / c}$ and $v_{s / c}$ are the values close to the spacecraft, and $T_{\text {mid }}$ and $v_{\text {mid }}$ are intermediate values. The temperature and velocity values are estimated from the measured parameters of the MIRO spectra (continuum temperature, $T_{c}$, the saturated $\mathrm{H}_{2}{ }^{16} \mathrm{O}$ line core, $T_{s}$, and the Doppler shift of the $\mathrm{H}_{2}{ }^{18} \mathrm{O}$ line, $v_{\text {exp }}$ ). 


\begin{tabular}{|c|c|c|c|}
\hline Knot & Altitude $(\mathrm{m})$ & Temperature $(\mathrm{K})$ & Velocity $(\mathrm{km} / \mathrm{s})$ \\
\hline 1 & 5.5 & $T_{g r}$ & $v_{g r}$ \\
2 & 100 & $T_{g r}$ & $v_{g r}$ \\
3 & $500-800$ & $\frac{T_{g r}-T_{s / c}}{2}$ & $\frac{v_{g r}+v_{s / c}}{2}$ \\
4 & $1000-5600$ & $T_{s / c}$ & $v_{s / c}$ \\
5 & $H_{s / c}$ & $T_{s / c}$ & $v_{s / c}$ \\
\hline
\end{tabular}

Table 6.1: Co-ordinates for the knots of the a priori spline functions of the temperature and velocity profiles. $T_{g r}$ is the ground temperature close to the nucleus randomly chosen from the interval $T_{c}-10 \mathrm{~K} \leq T_{g r} \leq T_{c}+10 \mathrm{~K}$, where $T_{c}$ is the measured continuum temperature. $T_{s / c}$ is the spacecraft temperature randomly chosen from the interval $T_{s}-$ $15 \mathrm{~K} \leq T_{s / c} \leq T_{s}+15 \mathrm{~K}$, where $T_{s}$ is the measured temperature of the saturated $\mathrm{H}_{2}{ }^{16} \mathrm{O}$ line core. $v_{g r}$ is the expansion velocity at the nucleus randomly chosen from the interval $0 \mathrm{~km} / \mathrm{s} \geq v_{g r} \geq-0.4 \mathrm{~km} / \mathrm{s} . v_{s / c}$ is the spacecraft expansion velocity randomly chosen from the interval $v_{\text {exp }}+0.1 \mathrm{~km} / \mathrm{s} \geq v_{s / c} \geq v_{\text {exp }}-0.1 \mathrm{~km} / \mathrm{s}$, where $v_{\text {exp }}$ is the (negative) measured Doppler shift expansion velocity in the direction of the spacecraft. $H_{s / c}$ is the spacecraft altitude. The altitudes for knots 3 and 4 are randomly chosen from the given intervals.

The fourth knot has the same values for temperature and velocity as the fifth knot, $T_{s / c}$ and $v_{s / c}$, but this time, the altitude of this point is randomly chosen between $1000-5600 \mathrm{~m}$.

Finally, the third knots for temperature and velocity are randomly chosen to be in the interval 500-800 m and they take values of $T_{\text {mid }}$ and $v_{\text {mid }}$ where $T_{\text {mid }}=\frac{T_{g r}-T_{s / c}}{2}$ and $v_{\text {mid }}=\frac{v_{g r}+v_{s / c}}{2}$.

The co-ordinates of the knots are randomly chosen within their respective intervals for two main reasons. The first is that the measured atmospheric parameters from the MIRO data $\left(T_{c}, T_{s}\right.$ and $\left.v_{\text {exp }}\right)$ are only proxies for the atmospheric values that they represent. In addition, these parameters have their own error bars.

Secondly, by setting the knots within these limits, a series of a priori profiles can be generated. The inversion can then be performed multiple times, each time starting from a different a priori profile with different coordinates for the knots. In this way, we can quantify the dependence of the solution on the a priori parameters and hence assess the error on the retrieval. The a priori temperature and velocity profiles are randomly created by choosing $T_{c}, T_{s}$ and $v_{\text {exp }}$ from within their uncertainty ranges and by randomly selecting the third and fourth knot altitudes. The variety in a priori starting profiles will be useful later when we want to quantify the error on our retrieval solutions.

The shape of the density profile is estimated from DSMC modelling of the inner coma region (Marschall et al. 2016), behaving with an approximate $\frac{1}{r^{2}}$ dependence above a few hundred metres and having a reasonably constant density below this altitude. The density profile is scaled so that the integrated column density under the curve is equal to the column density, $N$, as estimated from the line area ratios of the $\mathrm{H}_{2}{ }^{16} \mathrm{O}$ and $\mathrm{H}_{2}{ }^{18} \mathrm{O}$ lines. The density profile is only scaled in the inversion process so only one scaling parameter is retrieved to describe the behaviour of the density. The grey dotted lines in Figure 6.3 give an example of the a priori profiles generated in this way. Again, there is uncertainty on the measurement $N$, so the a priori density profile has a randomly chosen integrated 
column density within the error bars of $N$ - a factor of about 2-3 (Marshall et al. 2017).

Each profile is interpolated to have 31 grid points and thus 30 cells. The first two grid points are at altitudes of $5.5 \mathrm{~m}$ and $100 \mathrm{~m}$ and after that, the remaining 29 points are distributed logarithmically until the altitude of the spacecraft. Using a purely logarithmic grid gives too many altitude points very close to the nucleus (within $100 \mathrm{~m}$ ) where the sensitivity in the measurements is not so high.

\subsubsection{Creating $K, S_{a}$ and $S_{e}$}

The next step involves creating the weighting functions or kernel, $K$, the covariance matrix $S_{a}$, and the measurement error covariance matrix, $S_{e}$.

$K$ is made by perturbing the elements in the a priori profiles. For the velocity and temperature profile, a small perturbation ( $1 \mathrm{~K}$ in temperature and $20 \mathrm{~m} / \mathrm{s}$ in velocity) is made at each altitude grid point and the density profile is perturbed by a single scaling factor (0.1). In this way, 61 perturbed profiles are made and each one is run through a radiative transfer equation in local thermal equilibrium to simulate the water spectral lines seen by MIRO. Each simulated pair of spectral lines is subtracted from the simulated lines of the unperturbed a priori profile to see how each perturbation has altered the spectral lines. The difference between the perturbed and unperturbed lines are stacked in a matrix. This is the kernel. It effectively shows how each perturbation produces changes in the spectral lines. This will allow the inversion process to know at which altitude changes can be made in the profiles to minimise the difference between the a priori spectra and the observed spectra. The LTE radiative transfer method used here is the same as that used in Lee et al. (2015) and Marshall et al. (2017), using an approximated escape probability approach (Bockelée-Morvan 1987, Litvak and Kuiper 1982).

$S_{e}$ takes the form of a square diagonal matrix with the $S_{e}[i, i]$ values assigned to be the error on each point of the spectra, $e_{i}$, and all other values are set to zero. The matrix looks like:

$$
S_{e}=\left[\begin{array}{ccccc}
e_{i} & 0 & 0 & \ldots & 0 \\
0 & e_{i} & 0 & \ldots & 0 \\
0 & 0 & e_{i} & \ldots & 0 \\
: & : & : & : & : \\
0 & 0 & 0 & \ldots & e_{i}
\end{array}\right]
$$

and it has dimensions of $m \times m$, where $m$ is the spectral resolution of the water lines.

$S_{a}$ is also a square matrix but it has dimensions of $p \times p$ where $p$ is the number of perturbations through the three atmospheres. For single beam inversion, $p=61$. The diagonals of the covariance matrix effectively describe how constrained the inversion is to the a priori. Off diagonal elements allow for consecutive altitude elements to be correlated. $S_{a}$ is not a diagonal matrix, and we instead use a Gaussian distribution centred on the diagonal element. The elements of $S_{a}$ are found using the equations:

$$
\begin{gathered}
S_{a}[j, k]=T_{s t d}^{2} \exp \left\{-\frac{(j-k)^{2}}{T_{c l}^{2}}\right\} ; \text { for } j, k<31 \\
S_{a}[j, k]=V_{s t d}^{2} \exp \left\{-\frac{(j-k)^{2}}{V_{c l}^{2}}\right\} ; \text { for } 31 \leq j, k<61
\end{gathered}
$$




$$
S_{a}[61,61]=D_{\text {std }}^{2}
$$

for each set of profiles. $T_{\text {std }}, V_{\text {std }}$ and $D_{\text {std }}$ are the standard deviations of the temperature and velocity, or how much each variable is allowed to deviate from the a priori. $T_{c l}$ and $V_{c l}$ are the respective correlation lengths or how many altitude grid points are correlated together, and $j$ and $k$ are elements of $S_{a}$. For the inversions performed here, $T_{\text {std }}=3 \mathrm{~K}, V_{\text {std }}=0.02 \mathrm{~km} / \mathrm{s}, D_{\text {std }}=0.1, T_{c l}=5$ and $V_{c l}=5$.

\subsubsection{Optimal estimation inversion}

In the third step, I use a regularised optimal inversion technique from Rodgers (2000), incorporating $K, S_{a}$ and $S_{e}$ as described above. The equation takes the form:

$$
\begin{gathered}
x_{i+1}=x_{i}+G\left[y-F\left(x_{i}\right)+K\left(x_{i}-x_{a}\right)\right] \\
G=\left(K S_{a} K^{T}+S_{e}\right)^{-1}
\end{gathered}
$$

where $i$ denotes the iteration, $G$ is called the gain matrix, $y$ is the observed spectra, $F\left(x_{i}\right)$ is the simulated spectra from the $x_{i}$ profiles, $x_{a}$ is the a priori profile, and $K^{T}$ is the transposed kernel. In Equation 6.5, the difference between observed and simulated spectra is minimised, and regularised by the smoothness of the profiles and the measurement error on the observed spectra. In the first iteration, $x_{i}=x_{a}$ but after that $x_{i}$ changes with each iteration. The process now starts again, taking the $x_{i+1}$ retrieved solution and creating a new kernel by perturbing the elements in these new profiles. Typically, the inversion converges on an answer in 5-10 steps.

It is possible to calculate an error covariance matrix from our inputs but because there are some unknown constraints in our inversion (the model parameter error, the forward model error), we prefer to quantify the error by examining the non-uniqueness of starting from each different a priori. By using the error on the measured parameters mentioned in Section 6.2.1, we create a family of a priori profiles and start the inversion from each one. Each a priori gives a different final answer and the variation in the retrieved answers gives us the error on each parameter.

For single beam inversion, a single set of temperature, velocity and density profiles are retrieved along a single line of sight. The a priori parameters are taken from the observed spectra. $x_{i}, x_{a}$ and $x_{i+1}$ have dimensions of $1 \times 61, y$ and $F\left(x_{i}\right)$ have dimensions of $1 \times p$ where $p=260$ for the spectral line resolution used here for the MIRO observations. $K$ is a $61 \times 260$ matrix and $S_{a}$ and $S_{e}$ are square matrices with 61 and 260 elements, respectively.

\subsection{Application}

In order to apply the inversion method to observed MIRO data, it is necessary to first check the performance of the parameter retrieval on a synthetic case study. In this way, the accuracy of the retrieved solutions can be quantified against prior determined true profiles. The geometry used for the synthetic case study and the MIRO case study is shown in Figure 6.2 and this is taken from April 9, 2016 at 23:48 when MIRO was $29.9 \mathrm{~km}$ away from the nucleus. The MIRO beam is shown in red and the temperature, velocity and density will be retrieved along this line of sight. 

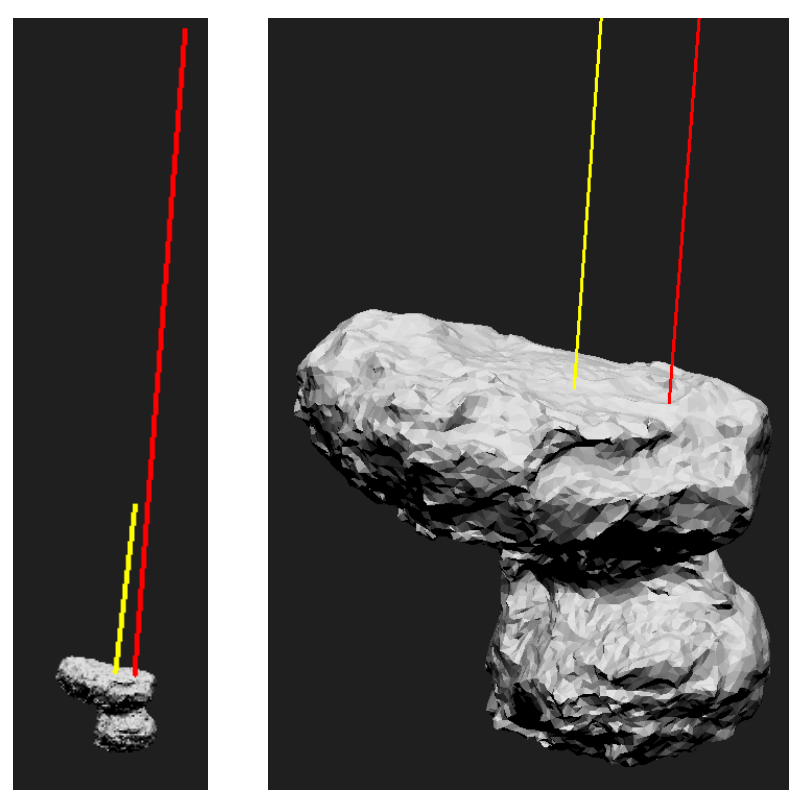

Figure 6.2: Left: Geometry of the MIRO observation on April 9, 2016 at 23:48. The red line shows the length of the MIRO beam from the spacecraft to the nucleus $(29.9 \mathrm{~km})$ and the yellow lines shows the direction of the sun. Right: zoomed in view of the geometry. MIRO is observing a part of the relatively flat Imhotep region which is well illuminated at this time.

\subsubsection{Synthetic case study}

For the synthetic case study, a coma atmosphere is created with profiles for temperature velocity and density. These are shown as black lines in the top panel of Figure 6.3. From these 'true' profiles, synthetic MIRO measurements are made for the $\mathrm{H}_{2}{ }^{16} \mathrm{O}$ and $\mathrm{H}_{2}{ }^{18} \mathrm{O}$ lines. These are shown in black in the top panel of Figure 6.4. Noise is added to the signal at a level comparable to that of an observation made with 12-minute integration time, as will be used for the MIRO data in the next section. The aim now is to retrieve the true synthetic profiles from the synthetic water line spectra using the inverse method described above.

The first step is to estimate the coma parameters $T_{c}, T_{s}, v_{\exp }$ and $N$ from the synthetic MIRO spectra. These are found to be: $26 \mathrm{~K}, 185 \mathrm{~K},-0.76 \mathrm{~km} / \mathrm{s}$ and $4.12 \times 10^{15} \mathrm{~cm}^{-2}$. These values are then used to create the 40 a priori profiles shown in grey dashed lines in the top panel of Figure 6.3. From these a priori profiles, the inversion begins and Equation 6.5 is solved. The retrieved solutions are shown in the yellow dashed lines in the top panel of Figure 6.3 and the difference between the retrieved profiles and the true profiles are shown in the middle panel of Figure 6.3, again in yellow dotted lines. The average of the retrieved solutions is then shown in green for the top and middle panels.

Qualitatively, the temperature profile and the velocity above $1 \mathrm{~km}$ is retrieved very well, and the average solution follows the true profiles, finding the inversion in both profiles. The solution for the density profile is also good, being reasonably close to the true profile. The difference between the average retrieved solution and the true profiles converges well around the zeroth line, shown in grey in the middle panels of Figure 6.3. The retrieved spectra are shown as coloured lines in Figure 6.4 and the residuals, the difference 

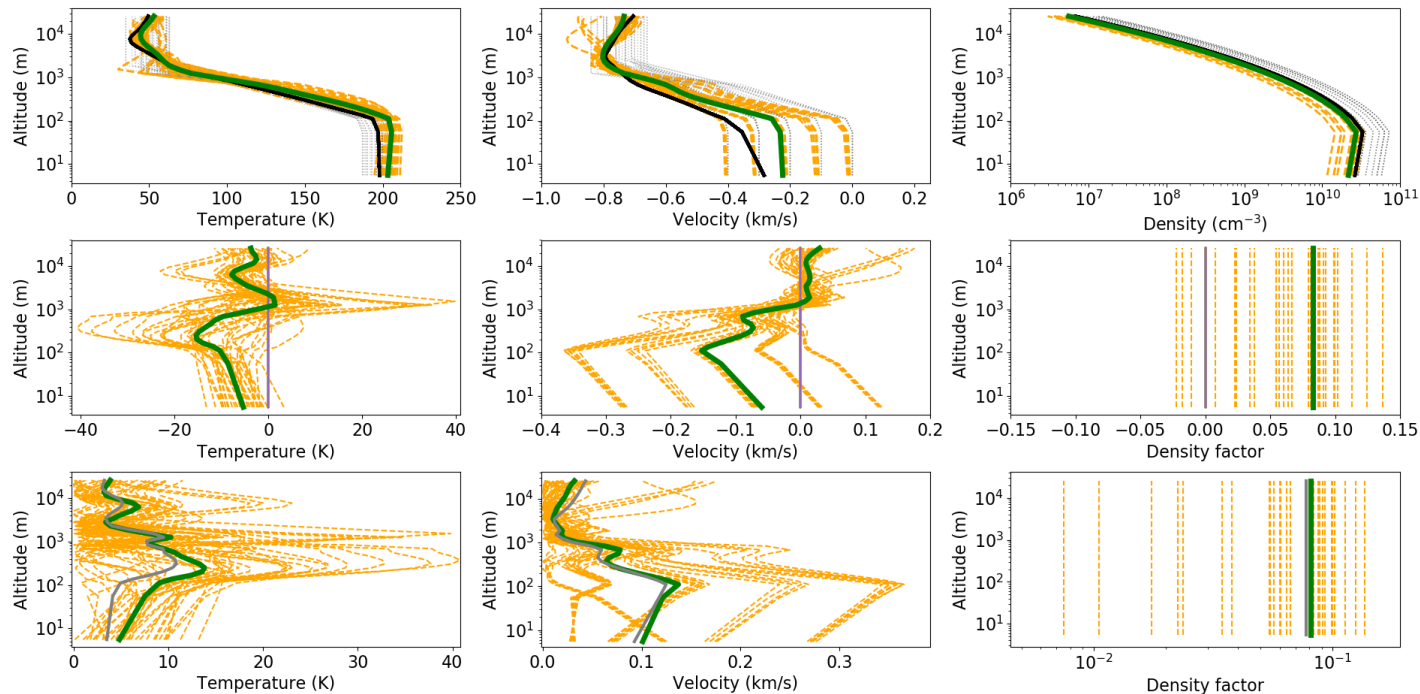

Figure 6.3: Top: Retrieved profiles for gas temperature, expansion velocity and water number density from the synthetic MIRO observations shown in Figure 6.4 using the viewing geometry of Figure 6.2. The black lines are the 'true' synthetic profiles, the grey lines show the a priori profiles, the yellow lines show the retrieved answers from each a priori and the green line is an average of all of these answers. Middle: The difference between the true and retrieved profiles for each inversion is shown in the yellow dotted lines. The green line is the average of all of these. Bottom: The absolute difference between the retrieved and true profiles are shown in yellow. The average of all of these profiles is shown in green and this quantity is called the bias. The standard deviation of the bias is called the variability and it is shown in grey.

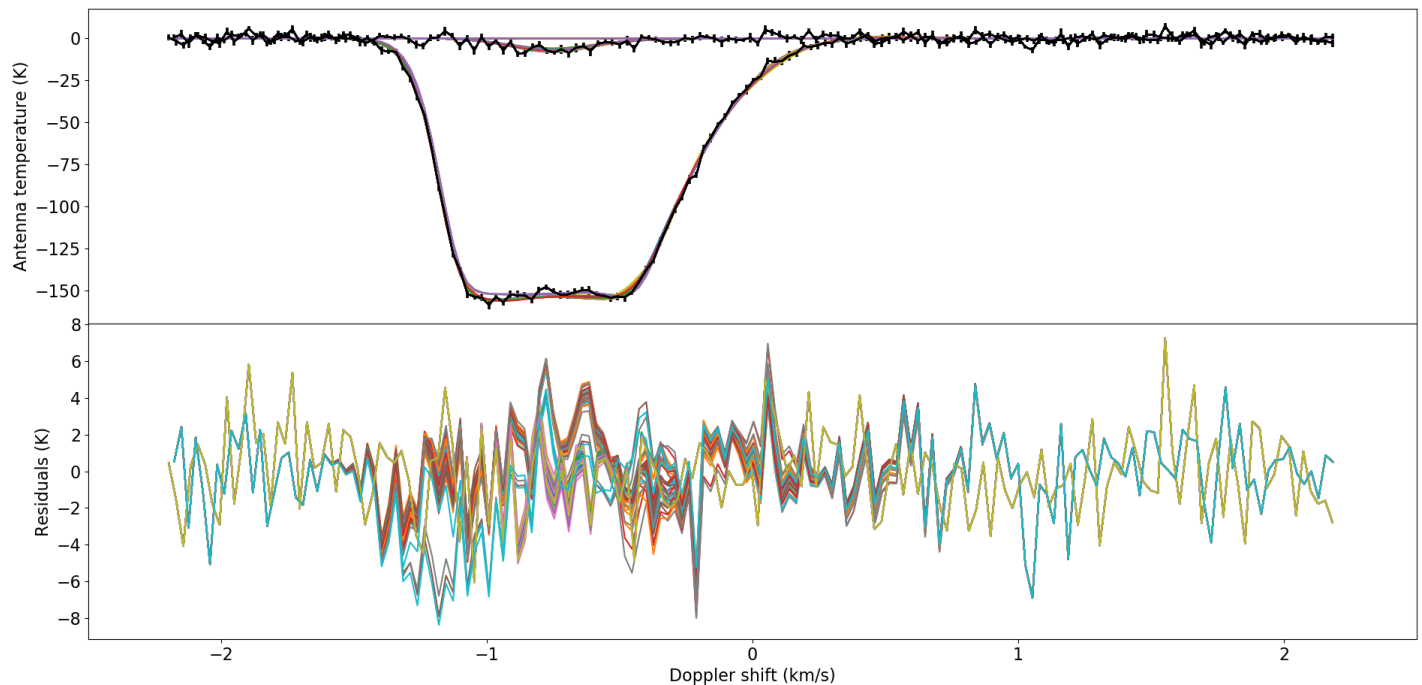

Figure 6.4: The synthetic MIRO spectra of $\mathrm{H}_{2}{ }^{16} \mathrm{O}$ and $\mathrm{H}_{2}{ }^{18} \mathrm{O}$ (black lines) and the retrieved spectra (coloured lines) along with the residuals (bottom panel). 
between the retrieved and measured spectra are all within $8 \mathrm{~K}$, with the biggest differences correlating to the noisier parts of the spectra.

The bottom panels of Figure 6.3 give the absolute difference between the average retrieved solution and the true solution, and from this we will quantify the quality of the retrieval by defining the errors. The yellow dotted lines again show the absolute difference between the true and retrieved profiles for each retrieval. The green line then gives the average of all of these. We call this the bias. For the temperature profile, only a small region at around $200 \mathrm{~m}$ has a bias greater than $10 \mathrm{~K}$ and in fact above $1250 \mathrm{~m}$ from the nucleus, the bias is less than $8 \mathrm{~K}$. The bias in the velocity profile is less than $30 \mathrm{~m} / \mathrm{s}$ from the true profile above $1250 \mathrm{~m}$ and often much better, particularly at around $3000 \mathrm{~m}$. The bias below $1000 \mathrm{~m}$ is not so good however, but this is to be expected. The MIRO water line spectra are not particularly sensitive to the velocity close to the nucleus. There is a small bias in the density profile, underestimating the density by a factor of about $8 \%$.

We also define the variability which is the standard deviation of the bias. It measures the variability in all of the retrievals. It is shown in grey in the bottom panel of Figure 6.3. Generally the $1 \sigma$ variability is close in value to the bias except in temperature less than $1000 \mathrm{~m}$ from the surface where the variability in the retrieved profiles decreases in comparison to the bias. We will use the bias to define the error on our retrieval from the real MIRO spectra.

\subsubsection{MIRO case study}

Now that we have assessed the performance of the inversion method, it can now be applied to real spectra measured by MIRO. Figure 6.5 shows the retrieved profiles for gas temperature, expansion velocity along the line of sight and water number density in the top panel, and the MIRO spectra underneath captured between April 9, 23:48 and April 10, 00:00, 2016. From the MIRO spectra, the measured coma parameters $\left(T_{c}, T_{s}, v_{\text {exp }}\right.$ and $N)$ are: $41 \mathrm{~K}, 190 \mathrm{~K},-0.66 \mathrm{~km} / \mathrm{s}$ and $1.04 \times 10^{15} \mathrm{~cm}^{-2}$. The grey a priori lines in the top panel of Figure 6.5 were made from these values. The retrieved solutions are shown in the yellow dashed lines and the average solution is given in green. The retrieved spectra are again shown as coloured lines in the bottom panel of the figure along with the residuals.

The retrieved solutions are reasonably consistent with little variability in the temperature and density profiles or the velocity profile above $\sim 1000 \mathrm{~m}$. The temperature profile increases from $190 \pm 5 \mathrm{~K}$ close to the surface $(5.5 \mathrm{~m})$ to $196 \pm 9 \mathrm{~K}$ at $111 \mathrm{~m}$ before decreasing to a minimum temperature of $50 \pm 3 \mathrm{~K}$ at $17200 \mathrm{~m}$. The temperature then stays reasonably constant up to the altitude of the spacecraft. The integrated column density under the number density profile takes a value of $(2.33 \pm 0.19) \times 10^{15} \mathrm{~cm}^{-2}$. The velocity at $1000 \mathrm{~m}$ is $-0.54 \pm 0.04 \mathrm{~km} / \mathrm{s}$ and increases to a value of $-0.70 \pm 0.01 \mathrm{~km} / \mathrm{s}$ at an altitude of $4200 \mathrm{~m}$. There is then a decrease towards a velocity minima of $-0.67 \pm 0.02 \mathrm{~km} / \mathrm{s}$ at $11500 \mathrm{~m}$ before finally increasing again to $-0.70 \pm 0.03 \mathrm{~km} / \mathrm{s}$ at the altitude of the spacecraft. The error on each parameter is taken from the bias given in the bottom panel of Figure 6.3. 

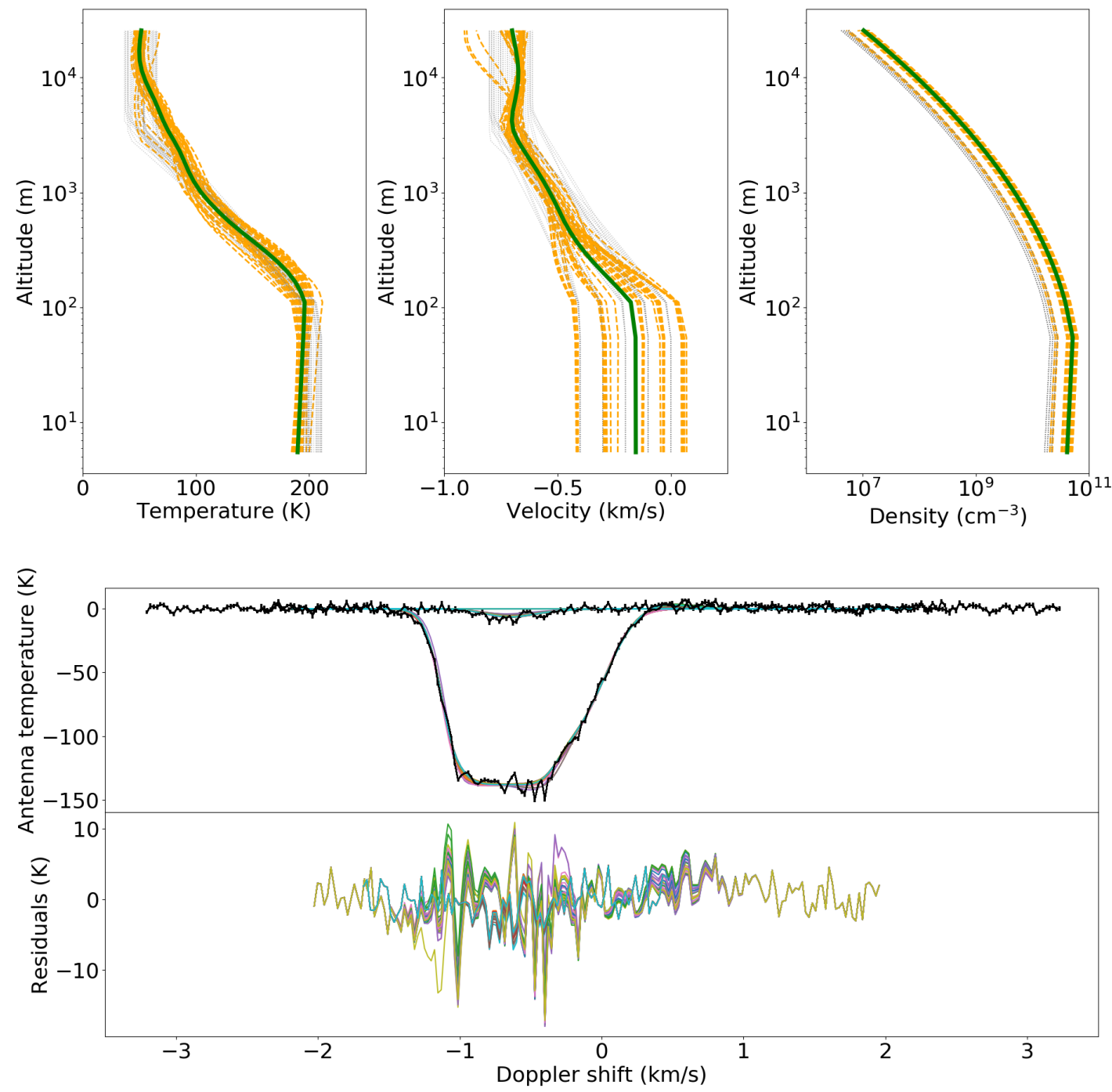

Figure 6.5: Top: Retrieved profiles for gas temperature, expansion velocity and water number density from the MIRO lines observed on April 9, 2016 at 23:48 with the viewing geometry shown in Figure 6.2. The grey lines show the a priori profiles, the yellow lines show the retrieved answers from each a priori and the green line is an average of all of these answers. Bottom: The MIRO spectra (black lines) and the retrieved spectra (coloured lines) along with the residuals. 


\subsection{Discussion}

In this section, we have shown how an iterative optimal estimation inversion method can be applied to MIRO data to retrieve coma profiles for gas temperature, expansion velocity and water number density. This method started from a priori profiles which were made using coma parameters estimated from the MIRO spectra, and through the regularised inversion method, I arrived at solutions for the profiles in the coma.

For the MIRO observation made on April 9, 2016 at 23:48, the temperature can be seen to decrease steadily from approximately $190 \mathrm{~K}$ close to the nucleus to $50 \mathrm{~K}$ at the spacecraft. The velocity increased from $0.54 \mathrm{~km} / \mathrm{s}$ at $1000 \mathrm{~m}$ to a maxima of $0.70 \mathrm{~km} / \mathrm{s}$ at before decreasing again to a minima of $0.67 \mathrm{~km} / \mathrm{s}$ at $11500 \mathrm{~m}$. The velocity then appeared to increase once more with a value of $0.70 \mathrm{~km} / \mathrm{s}$ close to the spacecraft. The water had a column density of $2.33 \times 10^{15} \mathrm{~cm}^{-2}$ for this observation.

The inversion method was tested with a synthetic case study to assess the errors on the parameters. The density profile has an error of about $8 \%$ at all altitudes as this profile has only one scaling parameter. Above $1250 \mathrm{~m}$ from the nucleus, the errors on temperature and velocity are quite small, less than $8 \mathrm{~K}$ and less than $30 \mathrm{~m} / \mathrm{s}$. Below this level, the error on the temperature can increase to $14 \mathrm{~K}$ mainly owing to the fact that the temperature profile is slightly overestimated at the altitudes where the temperature decreases rapidly. This can happen when the density is slightly underestimated as in this example. Below $1000 \mathrm{~m}$, the velocity profile is poorly retrieved. The MIRO spectra has little sensitivity here so the velocity cannot be found close to the nucleus.

The application of inverse methods will enable the dynamics of the inner coma region to be properly studied for the first time and allow structures like inversions to be discovered in the temperature and velocity profiles. By establishing the gas behaviour, it will be possible to characterise the temporal and spatial variations in the inner coma and study the activity in this unexplored region. Assessing the activity will help to answer some of the open questions about the physics of cometary outgassing.

Deriving coma profiles will help with comet modelling as it will enable the coma to be described from a data driven perspective for the first time, rather than with assumed parametrised profiles. In addition, the open questions about activity - distribution, driving species, dust lifting mechanism - can potentially be addressed by characterising the gas flow around the nucleus. Finally, physical aspects like photolytic heating or a Knudsen layer (Maali et al. 2016) could potentially be identified from the profiles which would enhance our understanding of the physical behaviour of outgassing in the coma.

These questions will be addressed in the future and not only from the nadir observations described here. It will also be possible to invert the spectra from limb observations. In addition, utilising the geometry of observations could potentially improve the accuracy on the retrieval, as is done in medical tomography. These will require new methods to be developed and tested before they can be applied to the MIRO data. 



\section{Discussion}

The science objectives for the MIRO instrument were outlined in Section 1.6 and these aims included: determining the abundances of major volatile species; characterising properties of the nucleus; and exploring the behaviour of the inner coma close to the surface (within $100 \mathrm{~km}$ ). Through this work, I have shown how MIRO is uniquely placed to provide constraints on the nucleus and the coma of comet 67P/Churyumov-Gerasimenko and achieve the goals set out before the beginning of the Rosetta mission. Here, the results are briefly summarised and put into a wider context.

\section{Water abundance}

In Chapter 3, I used the observed water spectral lines to measure the water production rate pre- and post-perihelion from the line area ratios of the $\mathrm{H}_{2}{ }^{16} \mathrm{O}$ and $\mathrm{H}_{2}{ }^{18} \mathrm{O}$ lines (Marshall et al. 2017). The abundance of sublimating water increased with decreasing heliocentric distance, as expected for an icy body, and reached a measured maximum of $(1.42 \pm 0.51) \times 10^{28} \mathrm{molec} / \mathrm{s}$ or $(426 \pm 153) \mathrm{kg} / \mathrm{s}$ on August 29, 2015. For comparison, the measured production rates 231 days before perihelion and 245 days after perihelion were, $(3.24 \pm 1.79) \times 10^{26} \mathrm{molec} / \mathrm{s}$, and $(1.72 \pm 0.54) \times 10^{26} \mathrm{molec} / \mathrm{s}$, respectively. The peak production from linear fitting of the data suggests that the peak production occurred $34 \pm 10$ days after perihelion.

During the perihelion passage, the MIRO results indicate that $67 \mathrm{P} /$ Churyumov-Gerasimenko lost $(2.4 \pm 1.1) \times 10^{9} \mathrm{~kg}$ of water, or $(1.2 \pm 0.6) \times 10^{10} \mathrm{~kg}$ of total material, assuming a dust-to-gas ratio, $\gamma=4$ (Rotundi et al. 2015). Taking the mass of the comet to be $1.0 \times 10^{13} \mathrm{~kg}$ (Sierks et al. 2015), this gave a mass loss of $0.12 \pm 0.06 \%$. With a small mass loss estimated for this apparition, it is tempting to assume that much of the nucleus interior is unprocessed during its lifetime, and the content beneath the surface may still be pristine. However, many aspects of the comet are still unknown. The dynamical history of 67P is not well determined more than 200 years ago and it is possible that it had close perihelion passages with the sun before (Maquet 2015), thus processing the material in the comet. In addition, the collisional history is also not well known and neither are the mechanisms which cause outbursts, possibly driven by volatile material in the deep interior. Comets are complex bodies and more must be done to properly understand their activity before conclusions can be drawn regarding solar system history.

The ROSINA instrument also provided an opportunity to measure the water production rate from Rosetta, using the onboard pressure sensor and mass spectrometer to determine the abundance of water around the spacecraft (Hansen et al. 2016). With a direct simulation Monte Carlo model, they find higher production rates than reported here. One 
reason for this may be the different acquisition techniques of the two instruments. MIRO is a remote sensing instrument and so observes material along its line of sight whereas ROSINA measures the material in the immediate vicinity. Differences between the results from both instruments may come from how both methods treat the behaviour of the gas between the nucleus and spacecraft, and raises the possibility of non- $\mathrm{r}^{2}$ dependence on the density, possibly due to extended sources. The discrepancies between measurements from the two instruments needs to be explained in future work.

In addition, the spatial resolution of MIRO enabled the water production to be limited to a determined region on the surface. The surface was found to be quite inhomogeneous in terms of outgassing, with Wosret, Neith and Bes being the regions where the highest measured production rates originated from. Notably, these are some of the southernmost regions and are particularly well illuminated around perihelion. MIRO did not observe high production rates from regions on the northern hemisphere, except for a couple of measurements in the Seth and Ash regions.

I also found the gradients for the change in the water production to have values of -3.8 and -4.3 , pre- and post-perihelion, respectively. So far though, no thermal modelling which is consistent with the observed MIRO water densities has been able to reproduce the detailed variation in $Q\left(r_{h}\right)$ and, as discussed in Chapter 4, the meaning of these slope values is not entirely clear. Crucially, the gradients of the production rate curves are also a function of heliocentric distance and a single value cannot describe the outgassing behaviour over large heliocentric distances. It is nearly impossible to disentangle the effects of nucleus shape, rotation axis orientation and activity distribution from the water production rate curve if these parameters are unknown, and when these three parameters are known as for 67P, the question now turns to how activity works to produce the observed outgassing. The inability of a simple sublimation model to reproduce features of the $67 \mathrm{P}$ water production curve suggest that there is inhomogeneous activity distributed on the surface but the cause of the inhomogeneity is still unknown. It could be explained by inhomogeneous ice coverage across the surface or due to dust coverage which has the effect of suppressing volatile sublimation.

\section{Nucleus properties}

Using the continuum channels of MIRO, I estimated the thermal inertia of the nucleus, as described in Chapter 5 (Marshall et al. 2018). In the context of all other Rosetta measurements, it seems likely that the surface has a low average value for the thermal inertia with $50 \mathrm{JK}^{-1} \mathrm{~m}^{-2} \mathrm{~s}^{-0.5}$ fitting within the error bars of most of the observations from MIRO, VIRTIS and MUPUS. Looking more widely, the derived values for other comets for the thermal inertias can also be low, with values less than $50 \mathrm{JK}^{-1} \mathrm{~m}^{-2} \mathrm{~s}^{-0.5}$ for unresolved nuclei (Groussin et al. 2018). Results from 67P (Marshall et al. 2018), 9P/Tempel and 103P/Hartley (Davidsson et al. 2013, Groussin et al. 2013) imply that higher thermal inertias may also be possible. This could be explained by variations in composition across the surface or by the fact that thermal inertia is a function of temperature, which varies across the surface based on the solar illumination. Comets appear then to have lower thermal inertias than many other solar system bodies, including near-Earth and Main Belt asteroids (Dell'Oro et al. 2007, Tanga et al. 2009). Looking to other solar system bod- 
ies, the Saturnian satellites (Howett et al. 2010), Pluto (Lellouch et al. 2017) and TransNeptunian Objects (Lellouch et al. 2013) appear to have very low thermal inertias too, less than $25 \mathrm{JK}^{-1} \mathrm{~m}^{-2} \mathrm{~s}^{-0.5}$ (Groussin et al. 2018).

As a result of low values for the thermal inertia, the temperature in the comet surface decreases quickly with depth. Depending upon the modelling used, after a couple of metres the temperature in the nucleus can be in the range 30-100 K (Marshall et al. 2018, $\mathrm{Hu}$ et al. 2017). The erosion rate during an orbit is on the order of decimetres or less (Gortsas et al. 2011, Keller et al. 2015, Groussin et al. 2018). These two findings imply that much of the nucleus could be unprocessed since formation if the interior parts of the comet remain very cold, and that samples from beneath the surface could be pristine remnants of the early solar system.

By combining this analysis with the VIRTIS data, it was also possible to map the small-scale thermal roughness on the shape model.

\section{The inner coma}

The application of inversion methods as described in Chapter 6 enables the retrieval of gas temperature, expansion velocity and water number density along the MIRO line of sight from spectral line observations. In this chapter an example inversion was performed for observations made on April 9, 2016. Starting from a priori profiles derived from coma parameters in the MIRO spectra, I arrived at profiles which show the steady decrease in temperature and the increase of expansion velocity from $1000 \mathrm{~m}$ above the surface to the spacecraft. I was also able to find the column density of water in the coma.

In the future, the widespread application of inversion methods will enable the inner coma to be probed and characterised in unprecedented detail and give new insights into the outgassing behaviour of comets. Through the analysis of the MIRO data, the temporal and spatial evolution of the coma activity can be studied and hence help to further our understanding of how activity works. Activity is generally not well understood - what drives it, which species dominate and how the material is lifted. Additionally, the retrieved profiles can help to establish the flow field around the nucleus. It may then be possible to assess the physics of the coma like a potential Knudsen layer close to the surface. The profiles could also reveal any photolytic heating or extended sources of water in the coma.

It is also possible that certain viewing geometries may be able to help improve the accuracy of the retrieval approach. Several observations made over a short period time which view the same volume of the coma from different perspectives could be inverted together to retrieve a single set of parameter profiles. This would be similar to tomographical methods employed in medical studies. Additionally, consecutive measured could be correlated together, thereby further regularising the problem.

\section{Concluding remarks}

MIRO provided an opportunity to probe the activity of comet 67P/Churyumov-Gerasimenko as part of the Rosetta mission and it has already revealed inhomogeneities in the spatial and temporal outgassing from the surface and in the coma. Much of the MIRO dataset has not been investigated yet, with most results so far coming 
from the early part of the mission. As a result, the MIRO dataset will be a valuable resource to use in any attempts to properly understand the physical behaviour of cometary activity. Hopefully there are still many exciting discoveries to come from the comet. 


\section{Bibliography}

Acton, C. H.: 1996, Planetary and Space Science 44(1), 65

A'Hearn, M. F., Belton, M., Delamere, W., Kissel, J., Klaasen, K., McFadden, L., Meech, K., Melosh, H., Schultz, P., Sunshine, J., et al.: 2005, Science

A'Hearn, M. F., Belton, M. J., Delamere, W. A., Feaga, L. M., Hampton, D., Kissel, J., Klaasen, K. P., McFadden, L. A., Meech, K. J., Melosh, H. J., et al.: 2011, Science 332(6036), 1396

Altwegg, K., Balsiger, H., Bar-Nun, A., Berthelier, J.-J., Bieler, A., Bochsler, P., Briois, C., Calmonte, U., Combi, M., De Keyser, J., et al.: 2015, Science 347(6220), 1261952

Baertschi, P.: 1976, Earth and Planetary Science Letters 31(3), 341

Bauduin, S., Irwin, P. G., Lellouch, E., Cottini, V., Moreno, R., Nixon, C., Teanby, N., Ansty, T., and Flasar, F.: 2018, Icarus 311, 288

Bertaux, J.-L.: 2015, Astronomy $\mathcal{E}$ Astrophysics 583, A38

Bertaux, J.-L., Combi, M., Quémerais, E., and Schmidt, W.: 2014, Planetary and Space Science 91, 14

Bibring, J.-P., Langevin, Y., Carter, J., Eng, P., Gondet, B., Jorda, L., Le Mouélic, S., Mottola, S., Pilorget, C., Poulet, F., and Vincendon, M.: 2015, Science 349(2)

Biele, J., Ulamec, S., Maibaum, M., Roll, R., Witte, L., Jurado, E., Muñoz, P., Arnold, W., Auster, H.-U., Casas, C., et al.: 2015, Science 349(6247), aaa9816

Bieler, A., Altwegg, K., Balsiger, H., Berthelier, J.-J., Calmonte, U., Combi, M., De Keyser, J., Fiethe, B., Fougere, N., Fuselier, S., et al.: 2015, Astronomy $\mathcal{E}$ Astrophysics 583, A7

Biermann, L.: 1951, Zeitschrift fur Astrophysik 29, 274

Birkebak, R. C.: 1972, The moon 4(1-2), 128

Biver, N., Bockelée-Morvan, D., Colom, P., Crovisier, J., Germain, B., Lellouch, E., Davies, J., Dent, W., Moreno, R., Paubert, G., et al.: 1997, Earth, Moon, and Planets 78(1-3), 5 
Biver, N., Hofstadter, M., Gulkis, S., Bockelée-Morvan, D., Choukroun, M., Lellouch, E., Schloerb, F., Rezac, L., Ip, W., Jarchow, C., et al.: 2015, Astronomy E Astrophysics 583, A3

Biver, N., Hofstadter, M., von Allmen, P., Bockelee-Morvan, D., Choukroun, M., Gulkis, S., Lee, S., Schloerb, F. P., Rezac, L., Leyrat, C., et al.: 2016, in AAS/Division for Planetary Sciences Meeting Abstracts, Vol. 48

Blundell, S. J. and Blundell, K. M.: 2009, Concepts in thermal physics, OUP Oxford

Bockelée-Morvan, D.: 1987, Astronomy and Astrophysics 181, 169

Bockelée-Morvan, D.: 2011, Proceedings of the International Astronomical Union 7(S280), 261

Bockelee-Morvan, D., Biver, N., Crovisier, J., de Val-Borro, M., Fulton, T., Hartogh, P., Hutsemékers, D., Jarchow, C., Jehin, E., Kidger, M., et al.: 2010, in Bulletin of the American Astronomical Society, Vol. 42, p. 946

Bockelée-Morvan, D., Debout, V., Erard, S., Leyrat, C., Capaccioni, F., Filacchione, G., Fougere, N., Drossart, P., Arnold, G., Combi, M., et al.: 2015, Astronomy E Astrophysics 583, A6

Bockelée-Morvan, D. and Gérard, E.: 1984, Astronomy and Astrophysics 131, 111

Bockelée-Morvan, D., Gautier, D., Hersant, F., Huré, J.-M., and Robert, F.: 2002, Astronomy $\mathcal{F}$ Astrophysics 384(3), 1107

Boice, D., Soderblom, L., Britt, D., Brown, R., Sandel, B., Yelle, R., Buratti, B., Oberst, J., Thomas, N., et al.: 2000, Earth, Moon, and Planets 89(1-4), 301

Bondarenko, Y. S. and Medvedev, Y. D.: 2011, Solar System Research 45(4), 330

Brouet, Y., Levasseur-Regourd, A. C., Sabouroux, P., Encrenaz, P., Thomas, N., Heggy, E., and Kofman, W.: 2015, Astronomy and Astrophysics 583, A39

Brouet, Y., Levasseur-Regourd, A. C., Sabouroux, P., Neves, L., Encrenaz, P., Poch, O., Pommerol, A., Thomas, N., Kofman, W., Le Gall, A., Ciarletti, V., Hérique, A., Lethuillier, A., Carrasco, N., and Szopa, C.: 2016, Monthly Notices of the Royal Astronomical Society 462, S89

Brownlee, D.: 2014, Annual Review of Earth and Planetary Sciences 42, 179

Brownlee, D. E., Horz, F., Newburn, R. L., Zolensky, M., Duxbury, T. C., Sandford, S., Sekanina, Z., Tsou, P., Hanner, M. S., Clark, B. C., et al.: 2004, Science 304(5678), 1764

Calla, O. P. N. and Rathore, I. S.: 2012, Advances in Space Research 50, 1607

Campbell, M. J. and Ulrichs, J.: 1969, Journal of Geophysical Research 74, 5867 
Capaccioni, F., Coradini, A., Filacchione, G., Erard, S., Arnold, G., Drossart, P., De Sanctis, M., Bockelee-Morvan, D., Capria, M., Tosi, F., et al.: 2015, Science 347(6220), aaa0628

Capria, M. T., Capaccioni, F., Filacchione, G., Tosi, F., De Sanctis, M. C., Mottola, S., Ciarniello, M., Formisano, M., Longobardo, A., Migliorini, A., et al.: 2017, Monthly Notices of the Royal Astronomical Society 469(Suppl_2), S685

Chance, K. and Martin, R. V.: 2017, Spectroscopy and Radiative Transfer of Planetary Atmospheres, Oxford University Press

Choukroun, M., Keihm, S., Schloerb, F., Gulkis, S., Lellouch, E., Leyrat, C., von Allmen, P., Biver, N., Bockelée-Morvan, D., Crovisier, J., et al.: 2015, Astronomy $\mathcal{E}$ Astrophysics 583, A28

Christianson, J. R. and Brahe, T.: 1979, Isis 70(1), 110

Clark, B., Mason, L. W., and Kissel, J.: 1988, in Exploration of Halley's Comet, pp 779-784, Springer

Clube, S., Hoyle, F., Napier, W., and Wickramasinghe, N.: 1996, Astrophysics and Space Science 245(1), 43

Cochran, A.: 1985, The Astronomical Journal 90, 2609

Cochran, A. L., Levasseur-Regourd, A.-C., Cordiner, M., Hadamcik, E., Lasue, J., Gicquel, A., Schleicher, D. G., Charnley, S. B., Mumma, M. J., Paganini, L., et al.: 2015, Space Science Reviews 197(1-4), 9

Combes, M., Moroz, V., Crifo, J., Lamarre, J., Charra, J., Sanko, N., Soufflot, A., Bibring, J., Cazes, S., Coron, N., et al.: 1986, Nature 321(6067), 266

Coradini, A., Capaccioni, F., Drossart, P., Arnold, G., Ammannito, E., Angrilli, F., Barucci, A., Bellucci, G., Benkhoff, J., Bianchini, G., et al.: 2007, Space Science Reviews 128(1), 529

Cowan, J. J. and A'Hearn, M. F.: 1979, the Moon and the Planets 21(2), 155

Crovisier, J., Colom, P., Gérard, E., Bockelée-Morvan, D., and Bourgois, G.: 2002, Astronomy $\mathcal{E}$ Astrophysics 393(3), 1053

Crovisier, J., Encrenaz, T., Lyle, S., et al.: 2000, Comet Science, Cambridge University Press

Davidsson, B. J., Gutiérrez, P. J., Groussin, O., A’Hearn, M. F., Farnham, T., Feaga, L. M., Kelley, M. S., Klaasen, K. P., Merlin, F., Protopapa, S., et al.: 2013, Icarus 224(1), 154

Davidsson, B. J. R., Rickman, H., Bandfield, J. L., Groussin, O., Gutiérrez, P. J., Wilska, M., Capria, M. T., Emery, J. P., Helbert, J., Jorda, L., Maturilli, A., and Mueller, T. G.: 2015, 252, 1 
De Pater, I. and Lissauer, J. J.: 2015, Planetary sciences, Cambridge University Press

De Sanctis, M., Capaccioni, F., Ciarniello, M., Filacchione, G., Formisano, M., Mottola, S., Raponi, A., Tosi, F., Bockelée-Morvan, D., Erard, S., et al.: 2015, Nature 525(7570), 500

de Val-Borro, M., Bockelée-Morvan, D., Jehin, E., Hartogh, P., Opitom, C., Szutowicz, S., Biver, N., Crovisier, J., Lis, D., Rezac, L., et al.: 2014, Astronomy $\mathcal{E}$ Astrophysics 564, A124

de Val-Borro, M., Hartogh, P., Crovisier, J., Bockelée-Morvan, D., Biver, N., Lis, D., Moreno, R., Jarchow, C., Rengel, M., Szutowicz, S., et al.: 2010, Astronomy E Astrophysics 521, L50

Dell'Oro, A., Harris, A. W., Mottola, S., Mueller, M., et al.: 2007, Icarus 190(1), 236

Dones, L., Brasser, R., Kaib, N., and Rickman, H.: 2015, Space Science Reviews 197(14), 191

Dones, L., Weissman, P. R., Levison, H. F., and Duncan, M. J.: 2004, Comets II p. 153

Duncan, M., Levison, H., and Dones, L.: 2004, Comets II pp 193-204

El-Maarry, M. R., Thomas, N., Giacomini, L., Massironi, M., Pajola, M., Marschall, R., Gracia-Berná, A., Sierks, H., Barbieri, C., Lamy, P. L., et al.: 2015, Astronomy $\mathcal{E}$ Astrophysics 583, A26

El-Maarry, M. R., Thomas, N., Gracia-Berná, A., Pajola, M., Lee, J.-C., Massironi, M., Davidsson, B., Marchi, S., Keller, H., Hviid, S., et al.: 2016, Astronomy E Astrophysics 593, A110

Emerich, C., Lamarre, J., Moroz, V., Combes, M., Sanko, N., Nikolsky, Y. V., Rocard, F., Gispert, R., Coron, N., Bibring, J., et al.: 1988, in Exploration of Halley's Comet, pp 839-842, Springer

Farnham, T., Duxbury, T., and Li, J.: 2005, Shape models of Comet Wild 2, Technical report, SDU-CNAVCAM-5-WILD2-SHAPE-MODEL-V2. 0, NASA Planetary Data System

Farnham, T., Kelley, M., A'Hearn, M., Feaga, L., Bodewits, D., Sunshine, J., Wellnitz, D., and Wissler, S.: 2017, Icarus 284, 106

Farnham, T. and Thomas, P.: 2013a, PLATE SHAPE MODEL OF COMET 103P/HARTLEY 2 V1.0, Technical report, DIF-C-HRIV/MRI-5-HARTLEY2-SHAPEV1.0, NASA Planetary Data System

Farnham, T. and Thomas, P.: 2013b, PLATE SHAPE MODEL OF COMET 9P/TEMPEL 1 V2.0, Technical report, DIF-C-HRIV/ITS/MRI-5-TEMPEL1-SHAPE-MODEL-V2.0, NASA Planetary Data System 
Feaga, L. M., A’Hearn, M. F., Farnham, T. L., Bodewits, D., Sunshine, J. M., Gersch, A. M., Protopapa, S., Yang, B., Drahus, M., and Schleicher, D. G.: 2013, The Astronomical Journal 147(1), 24

Feldman, P. D., A'Hearn, M. F., and Festou, M. C.: 2004, in The New Rosetta Targets, pp 47-52, Springer

Ferrín, I.: 2010, Planetary and Space Science 58(3), 365

Festou, M., Keller, H., and Weaver, H.: 2004, Comets II pp 3-16

Filacchione, G., Capaccioni, F., Ciarniello, M., Raponi, A., Tosi, F., De Sanctis, M. C., Erard, S., Morvan, D. B., Leyrat, C., Arnold, G., et al.: 2016, Icarus 274, 334

Fink, U., Doose, L., Rinaldi, G., Bieler, A., Capaccioni, F., Bockelée-Morvan, D., Filacchione, G., Erard, S., Leyrat, C., Blecka, M., et al.: 2016, Icarus 277, 78

Fougere, N., Altwegg, K., Berthelier, J.-J., Bieler, A., Bockelee-Morvan, D., Calmonte, U., Capaccioni, F., Combi, M. R., De Keyser, J., Debout, V., et al.: 2016, Astronomy $\mathcal{E}$ Astrophysics 588, A134

Glassmeier, K.-H., Boehnhardt, H., Koschny, D., Kührt, E., and Richter, I.: 2007, Space Science Reviews 128(1-4), 1

Goldsmith, P. F. and Langer, W. D.: 1999, The Astrophysical Journal 517(1), 209

Gortsas, N., Kührt, E., Motschmann, U., and Keller, H.: 2011, Icarus 212(2), 858

Gribbin, J. R.: 2004, The scientists: A history of science told through the lives of its greatest inventors, Random House Trade Paperbacks

Groussin, O., Attree, N., Brouet, Y., Ciarletti, V., Davidsson, B., Filacchione, G., Fischer, H.-H., Gundlach, B., Knapmeyer, M., Knollenberg, J., Koktanekova, R., Kührt, E., Leyrat, C., Marshall, D., Pelivan, I., Skorov, Y., Snodgrass, C., Spohn, T., and Tosi, F.: 2018, Space Science Reviews

Groussin, O. and Lamy, P.: 2003, Astronomy E Astrophysics 412(3), 879

Groussin, O., Sierks, H., Barbieri, C., Lamy, P., Rodrigo, R., Koschny, D., Rickman, H., Keller, H., A'Hearn, M., Auger, A.-T., et al.: 2015, Astronomy $\mathcal{E}$ Astrophysics 583, A36

Groussin, O., Sunshine, J., Feaga, L., Jorda, L., Thomas, P., Li, J.-Y., A’Hearn, M., Belton, M., Besse, S., Carcich, B., et al.: 2013, Icarus 222(2), 580

Guilbert-Lepoutre, A. and Jewitt, D.: 2011, The Astrophysical Journal 743(1), 31

Guilbert-Lepoutre, A., Rosenberg, E. D., Prialnik, D., and Besse, S.: 2016, Monthly Notices of the Royal Astronomical Society 462(Suppl 1), S146 
Gulkis, S., Allen, M., von Allmen, P., Beaudin, G., Biver, N., Bockelée-Morvan, D., Choukroun, M., Crovisier, J., Davidsson, B. J., Encrenaz, P., et al.: 2015, Science 347(6220), aaa0709

Gulkis, S., Frerking, M., Crovisier, J., Beaudin, G., Hartogh, P., Encrenaz, P., Koch, T., Kahn, C., Salinas, Y., Nowicki, R., et al.: 2007, Space Science Reviews 128(1-4), 561

Gulkis, S., Keihm, S., Kamp, L., Backus, C., Janssen, M., Lee, S., Davidsson, B., Beaudin, G., Biver, N., Bockelée-Morvan, D., et al.: 2010, Planetary and Space Science 58(9), 1077

Gulkis, S., Keihm, S., Kamp, L., Lee, S., Hartogh, P., Crovisier, J., Lellouch, E., Encrenaz, P., Bockelee-Morvan, D., Hofstadter, M., et al.: 2012, Planetary and Space Science 66(1), 31

Gundlach, B., Skorov, Y. V., and Blum, J.: 2011, Icarus 213(2), 710

Guzzo, M. and Lega, E.: 2015, Astronomy E Astrophysics 579, A79

Hampton, D. L., Baer, J. W., Huisjen, M. A., Varner, C. C., Delamere, A., Wellnitz, D. D., A'Hearn, M. F., and Klaasen, K. P.: 2005, Space Science Reviews 117(1-2), 43

Hanner, M., Tedesco, E., Tokunaga, A., Veeder, G., Lester, D., Witteborn, F., Bregman, J., Gradie, J., and Lebofsky, L.: 1985, Icarus 64(1), 11

Hansen, K. C., Altwegg, K., Berthelier, J.-J., Bieler, A., Biver, N., Bockelée-Morvan, D., Calmonte, U., Capaccioni, F., Combi, M., De Keyser, J., et al.: 2016, Monthly Notices of the Royal Astronomical Society 462(Suppl_1), S491

Hapke, B.: 2012, Theory of reflectance and emittance spectroscopy, Cambridge university press

Hartogh, P., Błęcka, M., Jarchow, C., Sagawa, H., Lellouch, E., de Val-Borro, M., Rengel, M., Medvedev, A., Swinyard, B., Moreno, R., et al.: 2010a, Astronomy E Astrophysics 521, L48

Hartogh, P., Crovisier, J., de Val-Borro, M., Bockelée-Morvan, D., Biver, N., Lis, D., Moreno, R., Jarchow, C., Rengel, M., Emprechtinger, M., et al.: 2010b, Astronomy $\mathcal{E}$ Astrophysics 518, L150

Hartogh, P. and Hartmann, G.: 1990, Measurement Science and Technology 1(7), 592

Haser, L.: 1957, Bulletin de la Societe Royale des Sciences de Liege 43, 740

Hässig, M., Altwegg, K., Balsiger, H., Bar-Nun, A., Berthelier, J.-J., Bieler, A., Bochsler, P., Briois, C., Calmonte, U., Combi, M., et al.: 2015, Science 347(6220), aaa0276

Hogerheijde, M. R. and van der Tak, F. F.: 2000, Astronomy and Astrophysics 362, 697

Howett, C., Spencer, J., Pearl, J., and Segura, M.: 2010, Icarus 206(2), 573 
Hu, X., Shi, X., Sierks, H., Fulle, M., Blum, J., Keller, H., Kührt, E., Davidsson, B., Güttler, C., Gundlach, B., et al.: 2017, Astronomy $\mathcal{E}$ Astrophysics 604, A114

Huber, M. C. and Schwehm, G.: 1991, Space Science Reviews 56(1-2), 109

Huebner, W. F., Benkhoff, J., Capria, M.-T., Coradini, A., De Sanctis, C., Orosei, R., and Prialnik, D.: 2006, Heat and gas diffusion in comet nuclei, International Space Science Institute

Jabeen, I. and Kusakabe, M.: 1997, Chemical Geology 143(1-2), 115

Janssen, M. A.: 1994, Atmospheric remote sensing by microwave radiometry, WileyInterscience Publication

Jorda, L., Gaskell, R., Capanna, C., Hviid, S., Lamy, P., Ďurech, J., Faury, G., Groussin, O., Gutiérrez, P., Jackman, C., et al.: 2016, Icarus 277, 257

Jutzi, M. and Asphaug, E.: 2015, Science 348(6241), 1355

Jutzi, M. and Benz, W.: 2017, Astronomy $\mathcal{E}$ Astrophysics 597, A62

Kaneda, E., Ashihara, O., Shimizu, M., Takagi, M., and Hirao, K.: 1986, Nature 321(6067s), 297

Karttunen, H., Kröger, P., Oja, H., Poutanen, M., and Donner, K. J.: 2016, Fundamental astronomy, Springer

Keihm, S., Tosi, F., Kamp, L., Capaccioni, F., Gulkis, S., Grassi, D., Hofstadter, M., Filacchione, G., Lee, S., Giuppi, S., et al.: 2012, Icarus 221(1), 395

Keller, H. U., Barbieri, C., Lamy, P., Rickman, H., Rodrigo, R., Wenzel, K.-P., Sierks, H., A'Hearn, M. F., Angrilli, F., Angulo, M., et al.: 2007, Space Science Reviews 128(1-4), 433

Keller, H. U., Mottola, S., Davidsson, B., Schröder, S., Skorov, Y., Kührt, E., Groussin, O., Pajola, M., Hviid, S. F., Preusker, F., et al.: 2015, Astronomy $\mathcal{E}$ Astrophysics 583, A34

King, J. I.: 1952, Journal of Meteorology 9(5), 311

Kofman, W., Herique, A., Barbin, Y., Barriot, J.-P., Ciarletti, V., Clifford, S., Edenhofer, P., Elachi, C., Eyraud, C., Goutail, J.-P., et al.: 2015, Science 349(6247), aab0639

Krankowsky, D., Lämmerzahl, P., Herrwerth, I., Woweries, J., Eberhardt, P., Dolder, U., Herrmann, U., Schulte, W., Berthelier, J., Illiano, J., et al.: 1986, Nature 321(6067), 326

Krolikowska, M.: 2003, Acta Astronomica 53, 195

Kührt, E.: 1999, Space Science Reviews 90(1-2), 75

Kührt, E., Giese, B., Keller, H. U., and Ksanfomality, L. V.: 1992, 96, 213 
Laeuter, M., Kramer, T., Rubin, M., and Altwegg, K.: 2018, arXiv preprint arXiv:1804.06696

Lamy, P., Toth, I., Groussin, O., Jorda, L., Kelley, M., and Stansberry, J.: 2008, Astronomy $\mathcal{E}$ Astrophysics 489(2), 777

Lamy, P., Toth, I., Weaver, H., Jorda, L., Kaasalainen, M., and Gutiérrez, P.: 2006, Astronomy $\mathcal{E}$ Astrophysics 458(2), 669

Lamy, P. L., Toth, I., Davidsson, B. J., Groussin, O., Gutiérrez, P., Jorda, L., Kaasalainen, M., and Lowry, S. C.: 2007, Space science reviews 128(1-4), 23

Langevin, Y., Hilchenbach, M., Ligier, N., Merouane, S., Hornung, K., Engrand, C., Schulz, R., Kissel, J., Rynö, J., Eng, P., et al.: 2016, Icarus 271, 76

Langmuir, I.: 1913, Physical review 2(5), 329

Le Roy, L., Altwegg, K., Balsiger, H., Berthelier, J.-J., Bieler, A., Briois, C., Calmonte, U., Combi, M. R., De Keyser, J., Dhooghe, F., et al.: 2015, Astronomy E Astrophysics 583, A1

Lee, S., Von Allmen, P., Allen, M., Beaudin, G., Biver, N., Bockelée-Morvan, D., Choukroun, M., Crovisier, J., Encrenaz, P., Frerking, M., et al.: 2015, Astronomy $\mathcal{E}$ Astrophysics 583, A5

Lellouch, E., Moreno, R., Müller, T., Fornasier, S., Santos-Sanz, P., Moullet, A., Gurwell, M., Stansberry, J., Leiva, R., Sicardy, B., et al.: 2017, Astronomy $\mathcal{E}$ Astrophysics 608, A45

Lellouch, E., Moreno, R., Orton, G., Feuchtgruber, H., Cavalié, T., Moses, J., Hartogh, P., Jarchow, C., and Sagawa, H.: 2015, Astronomy $\mathcal{E}$ Astrophysics 579, A121

Lellouch, E., Santos-Sanz, P., Lacerda, P., Mommert, M., Duffard, R., Ortiz, J., Müller, T., Fornasier, S., Stansberry, J., Kiss, C., et al.: 2013, Astronomy $\mathcal{E}$ Astrophysics 557, A60

Levison, H. and Dones, L.: 2014, Encyclopedia of the Solar System (Third Edition) pp 705-719

Levison, H. F.: 1996, in Completing the Inventory of the Solar System, Vol. 107, pp 173-191

Leyrat, C., Tosi, F., Capaccioni, F., Érard, S., Rousseau, B., Filacchione, G., Capria, M. T., De Sanctis, M. C., Bockelee-Morvan, D., Kührt, E., Schmitt, B., Migliorini, A., Kappel, D., and VIRTIS/Rosetta Team: 2016, in AAS/Division for Planetary Sciences Meeting Abstracts, Vol. 48 of AAS/Division for Planetary Sciences Meeting Abstracts, p. 219.02

Litvak, M. and Kuiper, E. R.: 1982, The Astrophysical Journal 253, 622 
Lowry, S., Duddy, S., Rozitis, B., Green, S. F., Fitzsimmons, A., Snodgrass, C., Hsieh, H. H., and Hainaut, O.: 2012, Astronomy $\mathcal{G}$ Astrophysics 548, A12

Maali, A., Colin, S., and Bhushan, B.: 2016, Nanotechnology 27(37), 374004

Mall, U., Altwegg, K., Balsiger, H., Bar-Nun, A., Berthelier, J.-J., Bieler, A., Bochsler, P., Briois, C., Calmonte, U., Combi, M. R., et al.: 2016, The Astrophysical Journal 819(2), 126

Maquet, L.: 2015, Astronomy $\mathcal{E}$ Astrophysics 579, A78

Marschall, R., Su, C., Liao, Y., Thomas, N., Altwegg, K., Sierks, H., Ip, W.-H., Keller, H., Knollenberg, J., Kührt, E., et al.: 2016, Astronomy E Astrophysics 589, A90

Marshall, D., Groussin, O., Vincent, J., Brouet, Y., Kappel, D., Arnold, G., Capria, M., Filacchione, G., Hartogh, P., Hofstadter, M., et al.: 2018, Astronomy E Astrophysics 616, A 122

Marshall, D., Hartogh, P., Rezac, L., von Allmen, P., Biver, N., Bockelée-Morvan, D., Crovisier, J., Encrenaz, P., Gulkis, S., Hofstadter, M., et al.: 2017, Astronomy $\mathcal{E}$ Astrophysics 603, A87

Massironi, M., Simioni, E., Marzari, F., Cremonese, G., Giacomini, L., Pajola, M., Jorda, L., Naletto, G., Lowry, S., El-Maarry, M. R., et al.: 2015, Nature 526(7573), 402

Mattei, E., Lauro, S. E., Vannaroni, G., Cosciotti, B., Bella, F., and Pettinelli, E.: 2014, Icarus 229, 428

Meech, K. and Svoren, J.: 2004, Comets II p. 317

Migliorini, A., Piccioni, G., Capaccioni, F., Filacchione, G., Bockelée-Morvan, D., Erard, S., Leyrat, C., Combi, M., Fougere, N., Crovisier, J., et al.: 2016, Astronomy $\mathcal{E}$ Astrophysics 589, A45

Morbidelli, A. and Rickman, H.: 2015, Astronomy E Astrophysics 583, A43

Mottola, S., Arnold, G., Grothues, H.-G., Jaumann, R., Michaelis, H., Neukum, G., Bibring, J.-P., Schröder, S., Hamm, M., Otto, K., et al.: 2015, Science 349(6247), aab0232

Olhoeft, G. R. and Strangway, D. W.: 1975, Earth and Planetary Science Letters 24, 394

Oort, J. H. et al.: 1950, Bulletin of the Astronomical Institutes of the Netherlands 11, 91

Ootsubo, T., Kawakita, H., Hamada, S., Kobayashi, H., Yamaguchi, M., Usui, F., Nakagawa, T., Ueno, M., Ishiguro, M., Sekiguchi, T., et al.: 2012, The Astrophysical Journal 752(1), 15

Panale, F. P. and Salvail, J. R.: 1984, Icarus 60(3), 476

Pätzold, M., Andert, T., Hahn, M., Asmar, S., Barriot, J.-P., Bird, M., Häusler, B., Peter, K., Tellmann, S., Grün, E., et al.: 2016, Nature 530(7588), 63 
Preusker, F., Scholten, F., Matz, K.-D., Roatsch, T., Hviid, S., Mottola, S., Knollenberg, J., Kührt, E., Pajola, M., Oklay, N., et al.: 2017, Astronomy $\mathcal{E}$ Astrophysics 607, L1

Preusker, F., Scholten, F., Matz, K.-D., Roatsch, T., Willner, K., Hviid, S., Knollenberg, J., Jorda, L., Gutiérrez, P. J., Kührt, E., et al.: 2015, Astronomy $\mathcal{F}$ Astrophysics 583, A33

Rengel, M., Sagawa, H., Hartogh, P., Lellouch, E., Feuchtgruber, H., Moreno, R., Jarchow, C., Courtin, R., Cernicharo, J., and Lara, L.: 2014, Astronomy E Astrophysics 561, A4

Richter, I., Koenders, C., Glassmeier, K., Tsurutani, B., and Goldstein, R.: 2011, Planetary and Space Science 59(8), 691

Rickman, H., Marchi, S., A’Hearn, M., Barbieri, C., El-Maarry, M. R., Güttler, C., Ip, W.-H., Keller, H., Lamy, P., Marzari, F., et al.: 2015, Astronomy $\mathcal{G}$ Astrophysics 583, A44

Robie, R. A., Hemingway, B. S., and Wilson, W. H.: 1970, Science 167(3918), 749

Rodgers, C. D.: 2000, Inverse methods for atmospheric sounding: theory and practice, Vol. 2, World scientific

Rodgers, S., Charnley, S., Huebner, W., and Boice, D.: 2004, Comets II pp 505-522

Rosenberg, E. D. and Prialnik, D.: 2010, Icarus 209(2), 753

Rotundi, A., Sierks, H., Della Corte, V., Fulle, M., Gutierrez, P. J., Lara, L., Barbieri, C., Lamy, P. L., Rodrigo, R., Koschny, D., et al.: 2015, Science 347(6220), aaa3905

Russo, N. D., DiSanti, M. A., Magee-Sauer, K., Gibb, E. L., Mumma, M. J., Barber, R. J., and Tennyson, J.: 2004, Icarus 168(1), 186

Russo, N. D., Mumma, M. J., DiSanti, M. A., Magee-Sauer, K., Novak, R., and Rettig, T. W.: 2000, Icarus 143(2), 324

Rybicki, G. B. and Hummer, D. G.: 1991, Astronomy and Astrophysics 245, 171

Sagdeev, R., Szabó, F., Avanesov, G., Cruvellier, P., Szabó, L., Szegő, K., Abergel, A., Balazs, A., Barinov, I., Bertaux, J.-L., et al.: 1986, Nature 321(6067s), 262

Saito, T., Yumoto, K., Hirao, K., Nakagawa, T., and Saito, K.: 1986, Nature 321(6067s), 303

Schleicher, D. G.: 2006, Icarus 181(2), 442

Schloerb, F. P., Keihm, S., von Allmen, P., Choukroun, M., Lellouch, E., Leyrat, C., Beaudin, G., Biver, N., Bockelée-Morvan, D., Crovisier, J., et al.: 2015, Astronomy $\mathcal{E}$ Astrophysics 583, A29

Schröder, S., Mottola, S., Arnold, G., Grothues, H.-G., Jaumann, R., Keller, H., Michaelis, H., Bibring, J.-P., Pelivan, I., Koncz, A., et al.: 2017, Icarus 285, 263 
Schulz, R.: 2009, Solar System Research 43(4), 343

Schulz, R., Hilchenbach, M., Langevin, Y., Kissel, J., Silen, J., Briois, C., Engrand, C., Hornung, K., Baklouti, D., Bardyn, A., et al.: 2015, Nature 518(7538), 216

Schwarz, G., Craubner, H., Delamere, A., Göbel, M., Gonano, M., Huebner, W., Keller, H., Kramm, R., Mikusch, E., Reitsema, H., et al.: 1988, in Exploration of Halley's Comet, pp 847-851, Springer

Schwehm, G. and Schulz, R.: 1999, in Laboratory astrophysics and space research, pp 537-546, Springer

Seargent, D. A.: 1982, Garden City, NY: Doubleday, 1982.

Sekanina, Z.: 1981, Annual Review of Earth and Planetary Sciences 9(1), 113

Sierks, H., Barbieri, C., Lamy, P. L., Rodrigo, R., Koschny, D., Rickman, H., Keller, H. U., Agarwal, J., A'Hearn, M. F., Angrilli, F., et al.: 2015, Science 347(6220), aaa1044

Skorov, Y. V., Rezac, L., Hartogh, P., and Keller, H. U.: 2017, Astronomy E Astrophysics 600, A 142

Snodgrass, C., Yang, B., and Fitzsimmons, A.: 2017, Astronomy $\mathcal{E}$ Astrophysics 605, A56

Spohn, T., Knollenberg, J., Ball, A., Banaszkiewicz, M., Benkhoff, J., Grott, M., Grygorczuk, J., Hüttig, C., Hagermann, A., Kargl, G., et al.: 2015, Science 349(6247), aab0464

Spohn, T., Seiferlin, K., Hagermann, A., Knollenberg, J., Ball, A. J., Banaszkiewicz, M., Benkhoff, J., Gadomski, S., Gregorczyk, W., Grygorczuk, J., et al.: 2007, Space Science Reviews 128(1-4), 339

Steckloff, J. K. and Jacobson, S. A.: 2016, Icarus 264, 160

Steckloff, J. K., Johnson, B. C., Bowling, T., Melosh, H. J., Minton, D., Lisse, C. M., and Battams, K.: 2015, Icarus 258, 430

Stephenson, F. R., Yau, K., and Hunger, H.: 1985, Nature 314(6012), 587

Stooke, P.: 2002, NASA Planetary Data System 166

Szabó, G. M., Kiss, L. L., Pál, A., Kiss, C., Sárneczky, K., Juhász, A., and Hogerheijde, M. R.: 2012, The Astrophysical Journal 761(1), 8

Tanga, P. et al.: 2009, Planetary and Space Science 57(2), 259

Thelen, A. E., Nixon, C., Chanover, N., Molter, E., Cordiner, M., Achterberg, R., Serigano, J., Irwin, P., Teanby, N., and Charnley, S.: 2018, Icarus 307, 380 
Thomas, N., Barbieri, C., Keller, H., Lamy, P., Rickman, H., Rodrigo, R., Sierks, H., Wenzel, K., Cremonese, G., Jorda, L., et al.: 2012, Planetary and Space Science 66(1), 96

Thomas, N. and Keller, H.: 1988, in Exploration of Halley's Comet, pp 843-846, Springer

Thomas, N., Sierks, H., Barbieri, C., Lamy, P. L., Rodrigo, R., Rickman, H., Koschny, D., Keller, H. U., Agarwal, J., A'Hearn, M. F., et al.: 2015, Science 347(6220), aaa0440

Twomey, S.: 2013, Introduction to the mathematics of inversion in remote sensing and indirect measurements, Vol. 3, Elsevier

Ulaby, F. T., Long, D. G., Blackwell, W. J., Elachi, C., Fung, A. K., Ruf, C., Sarabandi, K., Zebker, H. A., and Van Zyl, J.: 2014, Microwave radar and radiometric remote sensing, Vol. 4, University of Michigan Press Ann Arbor

Veverka, J., Klaasen, K., A'Hearn, M., Belton, M., Brownlee, D., Chesley, S., Clark, B., Economou, T., Farquhar, R., Green, S., et al.: 2013, Icarus 222(2), 424

Von Rosenvinge, T. T., Brandt, J. C., and Farquhar, R. W.: 1986, Science 232(4748), 353

Watson, K., Murray, B. C., and Brown, H.: 1962, Icarus 1(1-6), 317

Wedlund, C. S., Kallio, E., Alho, M., Nilsson, H., Wieser, G. S., Gunell, H., Behar, E., Pusa, J., and Gronoff, G.: 2016, Astronomy $\mathcal{E}$ Astrophysics 587, A154

Weissman, P. R. and Kieffer, H. H.: 1981, Icarus 47(3), 302

Whipple, F. L.: 1950, The Astrophysical Journal 111, 375

Wolters, A.: 1993, The Catholic Biblical Quarterly 55(4), 687

Yamada, T., Rezac, L., Larsson, R., Hartogh, P., Yoshida, N., and Kasai, Y.: 2018, Astronomy and Astrophysics, Accepted

Yeomans, D. and Chodas, P.: 1989, The Astronomical Journal 98, 1083

Zakharov, V., Bockelée-Morvan, D., Biver, N., Crovisier, J., and Lecacheux, A.: 2007, Astronomy $\mathcal{E}$ Astrophysics 473(1), 303

Zhen-Tao, X., Stephenson, F., and Yao-Tiao, J.: 1995, Quarterly Journal of the Royal Astronomical Society 36, 397 
Appendix 



\section{A Derivation of radiative transfer equation}

This is the derivation of the equation used for microwave remote sensing given in Equation from the standard definition of the radiative transfer equation (Equation 2.8). We start with this equation

$$
\frac{\mathrm{d} I_{v}(s)}{\mathrm{d} s}=j_{v}(s)-\alpha_{v}(s) I_{v}(s)
$$

and divide both sides by $\alpha_{v}(s)$

$$
\frac{1}{\alpha_{v}(s)} \frac{\mathrm{d} I_{v}(s)}{\mathrm{d} s}=\frac{j_{v}(s)}{\alpha_{v}(s)}-I_{v}(s)
$$

This can then be expressed as

$$
\frac{\mathrm{d} I_{v}(s)}{\mathrm{d} \tau}=S_{v}(s)-I_{v}(s)
$$

using the definition of the source function and the fact that $\mathrm{d} \tau=\alpha_{v}(s) \mathrm{d} s$. All the terms can then be multiplied by $\exp (\tau)$

$$
\exp (\tau) \frac{\mathrm{d} I_{v}(s)}{\mathrm{d} \tau}+I_{v}(s) \exp (\tau)=S_{v}(s) \exp (\tau)
$$

which can be simplified using the Product Rule

$$
\exp (\tau) \frac{\mathrm{d} I_{\nu}(s)}{\mathrm{d} \tau}+I_{\nu}(s) \exp (\tau)=\frac{\mathrm{d}}{\mathrm{d} \tau} I_{\nu}(s) \exp (\tau)
$$

to get

$$
\frac{\mathrm{d}}{\mathrm{d} \tau} I_{\nu}(s) \exp (\tau)=S_{v}(s) \exp (\tau)
$$

Writing $I_{v}(s) \exp (\tau)$ and $S_{v}(s) \exp (\tau)$ as $\hat{I}_{v}$ and $\hat{S}_{v}$, respectively, this now becomes

$$
\frac{\mathrm{d} \hat{I}_{v}}{\mathrm{~d} \tau}=\hat{S}_{v}
$$

This differential equation can be solved using

$$
\int_{\hat{I}_{v, 0}}^{I_{v}} \mathrm{~d} \hat{I}_{v}=\int_{0}^{\tau_{0}} \hat{S}_{v} \mathrm{~d} \tau
$$


where the right side can simply be redefined in terms of $S_{v}(s)$ and the left hand side becomes

$$
\int_{\hat{I}_{v, 0}}^{I_{\nu}} \mathrm{d} \hat{I}_{\nu}=\left[\hat{I}_{\nu}\right]_{\hat{I}_{v, 0}}^{I_{\nu}}=\hat{I}_{\nu}-\hat{I}_{\nu, 0}=I_{\nu} \exp \{\tau\}-I_{\nu, 0} \exp \{0\}
$$

which then leads to the form of the radiative transfer equation

$$
I_{v}=I_{v, 0} \exp \left(-\tau_{v}\left(s_{o}\right)\right)+\int_{0}^{s_{o}} S_{v} \exp \left(-\tau_{v}(s)\right) \alpha_{v}(s) d s
$$

that is commonly used in microwave remote sensing. 


\section{Publications}

\section{Refereed publications}

- Groussin, O., Attree, N., Brouet, Y., Ciarletti, V., Davidsson, B., Filacchione, G., Fischer, H.-H., Gundlach, B., Knapmeyer, M., Knollenberg, J., Kokotanekova, R., Kührt, E., Leyrat, C., Marshall, D., Pelivan, I., Skorov, Y., Snodgrass, C., Spohn, T., Tosi, F. "The thermal, mechanical, structural and dielectric properties of cometary nuclei after Rosetta" Space Science Reviews, under review

- Marschall, R., Rezac, L., Kappel, D., Su, C.C., Gerig, S.-B., Rubin, M., PinzonRodriguez, O., Marshall, D., Liao, Y., Herny, C., Arnold, G., Christou, C., Dadzie, K., Groussin, O., Hartogh, P., Jorda, L., Kührt, E., Mottola, S., Mousis, O., Preusker, F., Scholten, F., Theologou, P., Wu, J.-S., Altwegg, K., Rodrigo, R., Thomas, N. "A comparison of multiple Rosetta data sets and 3D model calculations of 67P/Churyumov-Gerasimenko coma around equinox (May 2015)" Icarus, under review

- Marshall, D., Rezac, L., Hartogh, P., Zhao, Y., Attree, N. "On the interpretation of heliocentric water production rates of comets" Astronomy E Astrophysics, Accepted

- Marshall, D., Groussin, O., Vincent, J.-B., Brouet, Y., Kappel, D., Hartogh, P., Jorda, L., Kührt, E., Mottola, S., Rezac, L., Rodrigo, R., Rodionov, S., Thomas, N. "Thermal inertia and roughness of the nucleus of comet 67P/Churyumov-Gerasimenko from MIRO and VIRTIS observations" Astronomy $\mathcal{E}$ Astrophysics 616 (2018): A122. https://doi.org/10.1051/0004-6361/201833104

- Marshall, D., Hartogh, P., Rezac, L., von Allmen, P., Biver, N., Bockelée-Morvan, D., Crovisier, J., Encrenaz, P., Gulkis, S., Hofstadter, M., Ip, W.-H., Jarchow, C., Lee, S., Lellouch, E. "Spatially resolved evolution of the local H2O production rates of comet 67P/Churyumov-Gerasimenko from the MIRO instrument on Rosetta" Astronomy E Astrophysics 603 (2017): A87. https://doi.org/10.1051/00046361/201730502

- Marshall, D., and Sadeghpour, H.R. "Simulating the formation of carbon-rich molecules on an idealized graphitic surface" Monthly Notices of the Royal Astronomical Society 455.3 (2015): 2889-2900. https://doi.org/10.1093/mnras/stv2524

\section{Conference presentations}


- Marshall, D., Rezac, L., Hartogh, P., and MIRO team "The inner coma of comet 67P/Churyumov-Gerasimenko as seen by MIRO" Asia Oceania Geosciences Society, Hawaii, June 2018 Contributed talk

- Marshall D., Groussin, O., Vincent, J.-B., Brouet, Y., Kappel, D., Arnold, G., Capria, M.T., Filacchione, G., Hartogh, P., Hofstadter, M., Ip, W.-H., Jorda, L., Kührt, E., Lellouch, E., Mottola, S., Rezac, L., Rodrigo, R., Rodionov, S., Schloerb, P. and Thomas, N. "Surface properties of comet 67P/Churyumov-Gerasimenko from MIRO and VIRTIS observations" Asia Oceania Geosciences Society, Hawaii, June 2018 Contributed poster

- Marshall, D., Groussin, O., Vincent, J.-B., Brouet, Y., Kappel, D., Hartogh, P., Jorda, L., Kührt, E., Mottola, S., Rezac, L., Rodrigo, R., Rodionov, S., Thomas, N. "Projection of data onto the shape model" Comets: Post 67P perspectives, ISSI workshop, Bern, Switzerland, Jan. 2018 Invited talk

- Marshall, D., Hartogh, P., Rezac, L. "The inner coma of comet 67P/ChuryumovGerasimenko" Rocks \& Stars II conference, Göttingen, Germany, Sep. 2017 Contributed poster

- Marshall, D., Hartogh, P., Rezac, L., von Allmen, P., Biver, N., Bockelée-Morvan, D., Crovisier, J., Encrenaz, P., Gulkis, S., Hofstadter, M., Ip, W.-H., Jarchow, C., Lee, S., Lellouch, E. "Cometary water outgassing: Spatially resolved evolution of the local $\mathrm{H} 2 \mathrm{O}$ production rate of comet $67 \mathrm{P} / \mathrm{Churyumov-Gerasimenko} \mathrm{from}$ MIRO/Rosetta" Asia Oceania Geosciences Society, Singapore, Aug. 2017 Contributed talk

- Marshall, D., Hartogh, P., Rezac, L. "Retrieving temperature and velocity profiles of comet 67P/Churyumov-Gerasimenko from MIRO/Rosetta water lines" Asia Oceania Geosciences Society, Singapore, Aug. 2017 Contributed poster

- Marshall, D., Hartogh, P., Rezac, L. "Water production rate of 67P/CG from the entire Rosetta/MIRO dataset with line area lookup-tables method" Water in the Universe conference, Noordwijk, The Netherlands, Apr. 2016 Contributed poster

- Marshall, D., and Sadeghpour, H. "Simulations of the Dynamics of Precursor Organic and Prebiotic Carbon-rich Moleculess." American Astronomical Society meeting, Seattle, USA, Jan. 2015 Contributed poster 


\section{Acknowledgements}

There are many people who I would like to thank for teaching, helping and supporting me through my PhD. First of all, I would like to thank my TAC members, Paul Hartogh and Stefan Dreizler, for guiding me through this work. I should thank Paul especially for giving me this opportunity to work on the Rosetta mission - it was really exciting to be working on this topic whilst the mission was ongoing and to see the data as it was coming in. I have learnt so much, so many thanks. I am also very grateful for all the other opportunities that you opened up for me (AOGS, Taiwan), but in particular being in the MiARD team was really fantastic. I met some really nice people through the project and it was great to be a part of it, learning so may interesting things from the wider community. Thanks to Nick Thomas and James Whitby for running the project so well.

I also need to say a huge thank you to Ladi Rezac. I have learnt so much from you over my PhD and you have been a massive help in everything I have done. I do not know where I would have been without you, so thanks for listening and answering all of my questions.

Thank you to the IMRPS co-ordinator, Sonja Schuh. I remember meeting all the way back in February 2015 during my interview week. It feels like a lifetime ago now, but it was a PhD-lifetime, I guess. I am very grateful that I had this opportunity through the IMPRS. Thank you!

Big thank you to Paul and Ladi for reading the drafts of this thesis in the final weeks before submission, and to Abbey and Felix for translating my summary into German! Danke Schön!

My time in Göttingen would have been nowhere near as fun without the amazing friends I made within the IMPRS group. I will not name you all - since there would be so many names, and no doubt I would forget some too - but thank you for being there and being magnificent. I find it immensely sad that the nature of working in academia means that friends come and go in such a short space of time, but I wish you all the best for the future, wherever it takes you.

I would like to thank my office mates who made our breaks ${ }^{1}$ enjoyable and fun. We sporcled our way through most of the website and, while I still do not know all the countries of the world, I at least learnt the capital of Kyrgyzstan. ${ }^{2}$.

For three years, I have enjoyed playing in the band MegaGauß. Again, there are too many people to name from our ever-changing lineup but thank you all for making Monday evenings so electric and magnetic ${ }^{3}$.

\footnotetext{
${ }^{1}$ they were short, I swear!

${ }^{2}$ Bishkek, I hope

${ }^{3}$ far greater than just $1 \mathrm{MG}$ !
} 
I would also like to thank my family for their love and support, my parents, Christine and Ian, and my brother, Chris. You were always there to listen to me and offer good advice, and I am so grateful for everything you have done for me throughout my life.

And finally, an Abb-knowledgement. Big thanks to my partner Abbey for supporting me and patiently putting up with my puns. It was exciting moving to Göttingen with you and I look forward to our next adventure. 


\section{Curriculum vitae}

\section{Personal information}

Name David Marshall

Date of Birth 03-December-1992

Place of Birth Norwich, UK

Nationality British

\section{Education}

- Ph.D.

$2015-2018$

Max Planck Institute for Solar System Research, Göttingen

University of Göttingen, Göttingen

- M.Phys

$2011-2015$

University of Southampton, Southampton, UK

Harvard-Smithsonian Center for Astrophysics, Boston, USA

- A levels

2009 - 2011

City of Norwich School, Norwich, UK

The Hewett School, Norwich, UK

Date: $13 / 11 / 2018$

Location: Göttingen

Signature: 